\title{
Controllable Proximity Effect in Superconducting Hybrid Devices
}

\section{Sergey Bakurskiy}

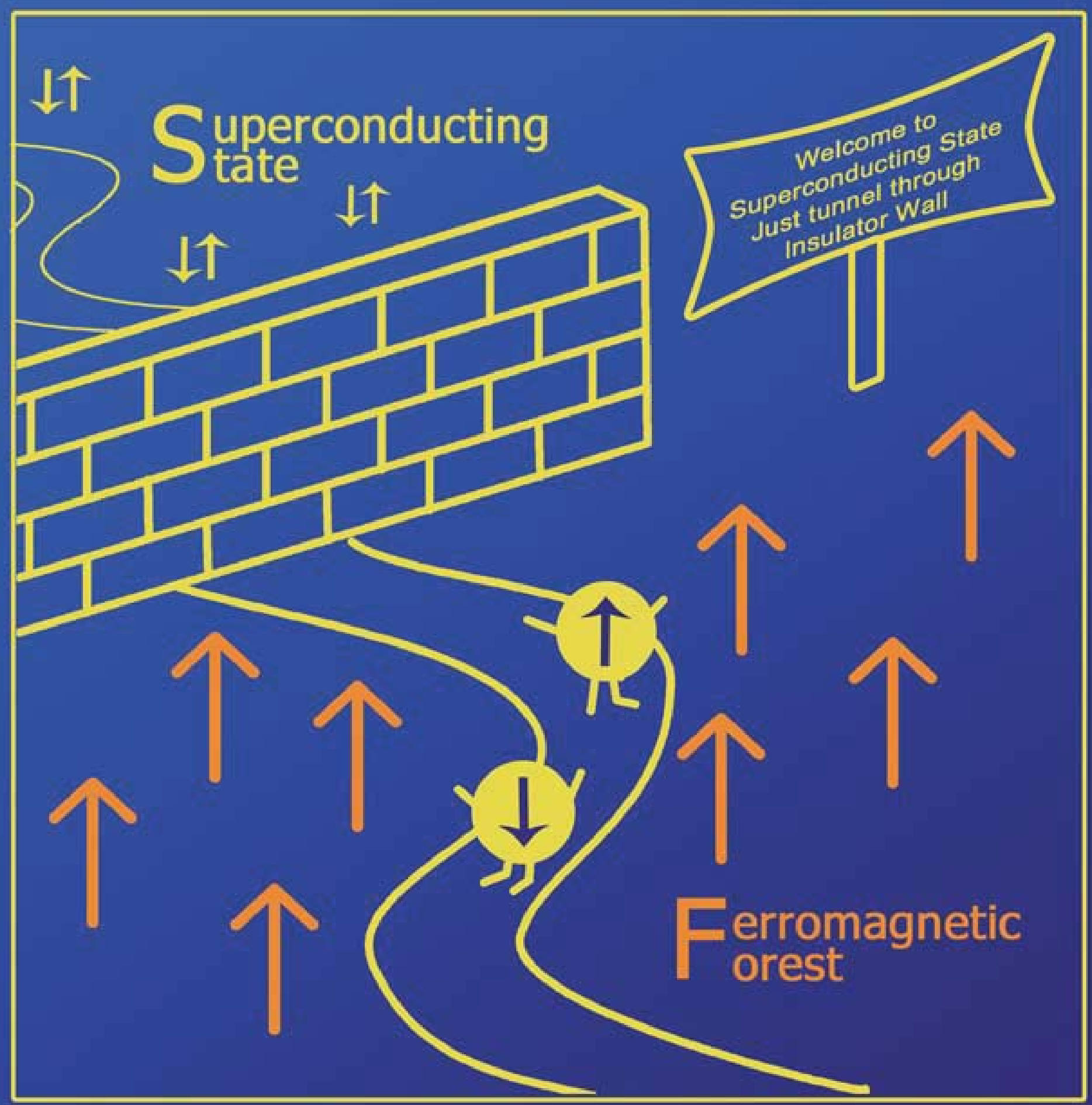


Controllable Proximity Effect in Superconducting Hybrid Devices

Ph.D. thesis of Sergey Bakurskiy 
Graduation committee:

$\begin{array}{lll}\begin{array}{l}\text { Chairman: } \\ \text { Secretary: }\end{array} & \text { Dean } & \text { University of Twente } \\ & \text { Dean } & \text { University of Twente } \\ \text { Promotor: } & \text { Prof.dr.ir. J.W.M. Hilgenkamp } & \text { University of Twente } \\ \text { Asst. Promotor: } & \text { dr. A.A. Golubov } & \text { University of Twente } \\ \text { Members: } & \text { Prof.dr.ir. H.J.W. Zandvliet } & \text { University of Twente } \\ & \text { Prof.dr.ir. A. Brinkman } & \text { University of Twente } \\ & \text { Prof.dr. J. Aarts } & \text { Leiden University } \\ & \text { Prof. M.Yu. Kupriyanov } & \text { Moscow State University }\end{array}$

The research described in this thesis was performed in the Faculty of Science and Technology and the MESA ${ }^{+}$Institute of Nanotechnology at the University of Twente (Enschede, The Netherlands), in collaboration with Moscow State University (Moscow, Russia) and Moscow Institute of Physics and Technology (Moscow region, Russia). This thesis was financially supported by the Dutch FOM and Ministry of Education and Science of the Russian Federation Grant No. 14Y.26.31.0007

Cover: Author's view on the proximity effect at Superconductor - Insulator - Ferromagnet interface

Printed by Ipskamp Drukkers, Enschede, The Netherlands.

ISBN: 978-90-365-3844-2

Copyright (c) 2015 by Sergey Bakurskiy

All rights reserved. No part of this work may be reproduced by print, photocopy or any other means without permission from the author. 


\title{
Controllable Proximity Effect in SuPERCONDUCTING HYBRID DEVICES
}

\author{
DISSERTATION
}

to obtain

the degree of doctor at the University of Twente,

on the authority of the rector magnificus,

prof. dr. H. Brinksma,

on account of the decision of the graduation committee,

to be publicly defended

on Friday, $27^{\text {th }}$ of February 2015 at 14:45

by

Sergey Bakurskiy

born on $1^{\text {st }}$ of July 1989

in Moscow, Russia 
The dissertation is approved by prof.dr.ir. J.W.M. Hilgenkamp, promotor dr. A.A. Golubov, assistant promotor 


\section{Contents}

Introduction $\quad 3$

Microscopic Theory of Superconductivity . . . . . . . . . . . . . . . . 3

Superconductor-Ferromagnet Hybrids . . . . . . . . . . . . . . . . . . 7

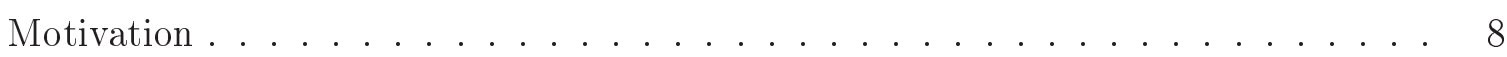

Contents of Chapters . . . . . . . . . . . . . . . . . . . 10

Chapter 1.Josephson $\varphi$-junctions based on structures with complex nor$\begin{array}{lr}\mathrm{mal} / \text { ferromagnet bilayer } & 19\end{array}$

1.1 Introduction . . . . . . . . . . . . . . . . . . . . . . . . . 19

1.2 CPR formation mechanisms . . . . . . . . . . . . . . . . . 21

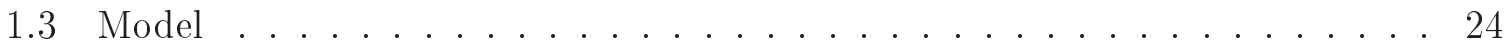

1.4 Ramp-type geometry . . . . . . . . . . . . . . . . . 26

1.4.1 Limit of small $L \ldots \ldots \ldots \ldots \ldots$. . . . . . . . . . . . . 28

1.4.2 Limit of intermediate $L \ldots \ldots \ldots$. . . . . . . . . . . . 29

$1.4 .3 \varphi$-state existence . . . . . . . . . . . . . 30

1.5 Overlap type geometry . . . . . . . . . . . . . . . 36

1.6 Ramp type overlap (RTO) junctions . . . . . . . . . . . . . . 37

1.7 Discussion . . . . . . . . . . . . . . . . . . . . 40

1.8 Appendix . . . . . . . . . . . . . . . . . . . . . . 42

1.8.1 Ramp type junctions. Limit of small $L \ldots \ldots$. . . . . . . . . . . 42

1.8.2 Ramp type junctions. Limit of intermediate $L \ldots \ldots$. . . . . . . . 45

1.8.3 Overlap SN-N-NS junctions . . . . . . . . . . . . . . 47

1.8.4 Solution in Ferromagnet Layer of RTO junction . . . . . . . . . . . . 49

Chapter 2.Theory of supercurrent transport in SIsFS Josephson junctions 57

2.1 Introduction . . . . . . . . . . . . . . . . . . . 57

2.2 Model of SIsFS Josephson device . . . . . . . . . . . . . . . . . 59

2.3 The high temperature limit $T \approx T_{C} \ldots \ldots \ldots \ldots$. . . . . . . . 61

2.3.1 Mode (1): SIs + sFS junction $d_{s} \geq d_{s c} \ldots \ldots \ldots$. . . . . . . 63 
2.3.1.1 Mode (1a): Switchable $0-\pi$ SIs junction . . . . . . . . 65

2.3.1.2 Mode (1b): sFS junction . . . . . . . . . . . . 67

2.3.2 Mode (2): SInFS junction $d_{s} \leq d_{s c} \ldots \ldots \ldots \ldots$. . . . . . 69

2.3.3 Current-Phase Relation . . . . . . . . . . . . . . . . . . 69

2.4 Arbitrary temperature . . . . . . . . . . . . . . . . . 72

2.4.1 Temperature crossover from 0 to $\pi$ states . . . . . . . . . . . 73

2.4.2 0 to $\pi$ crossover by changing the effective exchange energy in external magnetic field . . . . . . . . . . . . . . 76

2.5 Discussion . . . . . . . . . . . . . . . . . . 77

2.6 Appendix . . . . . . . . . . . . . . . . . 80

2.6.1 Boundary problem at $T \lesssim T_{C} \ldots \ldots \ldots$. . . . . . . 80

Chapter 3.Comparison of theory for SIsFS junctions with experimental $\begin{array}{ll}\text { data } & 88\end{array}$

3.1 Introduction . . . . . . . . . . . . . . . . . . . . . 88

3.2 Temperature dependence . . . . . . . . . . . . . . . . . . . 90

3.3 External magnetic field . . . . . . . . . . . . . . . . . . . . . . . . . . . . 92

3.4 Conclusion . . . . . . . . . . . . . . . . . . . . . . . 95

Chapter 4.Anomalous surface states at interfaces in p-wave superconductors 98

4.1 Introduction . . . . . . . . . . . . . . . . . . . . . . . 98

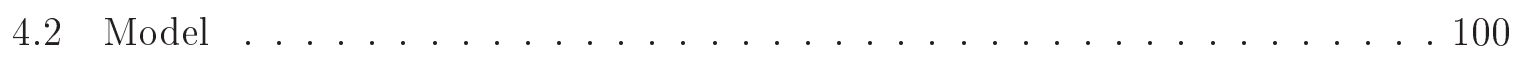

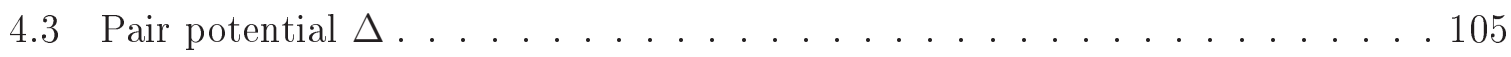

4.4 Pair amplitudes $f$ and $f^{+} \ldots \ldots \ldots \ldots \ldots \ldots \ldots$

4.5 Density of States . . . . . . . . . . . . . . . . . . . . 112

4.6 Conclusion . . . . . . . . . . . . . . . . . . . . 115

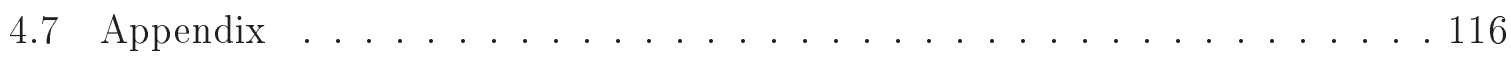

4.7 .1 Diffusive layer solution . . . . . . . . . . . . . . 116

4.7 .2 Symmetry relations . . . . . . . . . . . . . 118

$\begin{array}{lr}\text { Summary } & 126\end{array}$

$\begin{array}{ll}\text { Samenvatting (Summary in Dutch) } & 130\end{array}$

$\begin{array}{ll}\text { List of Publications } & 134\end{array}$

$\begin{array}{ll}\text { Acknowledgments } & 136\end{array}$ 


\section{Introduction}

\section{Microscopic Theory of Superconductivity}

Harbinger of the microscopic theory of superconductivity was the Froehlich theory [1] of the electron-phonon interaction, in which the positively charged lattice could lead to an effective attraction between two electrons with opposite spins. This interaction leads to the formation of pair correlated states (later named Cooper pairs) with a "negative" energy and integer spin. The existence of pairs drastically changes the electronic ground state of a material.

Further study of this phenomenon led to the emergence of the first microscopic theory of Bardeen-Cooper-Schrieffer (BCS) [2,3]. In the framework of BCS theory the Hamiltonian $H=\sum_{k} \zeta_{k} \hat{a}_{k}^{+} \hat{a}_{k}$ for an ideal electron gas in the second quantization representation was formulated taking into account the existence of pairing correlations. Then the model Hamiltonian of Bardeen-Cooper-Schrieffer (BCS Hamiltonian) takes the form:

$$
H=\sum_{k} \zeta_{k} \hat{a}_{k}^{+} \hat{a}_{k}+\sum_{k, i} V_{k}{ }_{i} \hat{a}_{k \uparrow}^{+} \hat{a}_{-k \downarrow}^{+} \hat{a}_{i \uparrow} \hat{a}_{-i \downarrow}
$$

Here $\hat{a}_{k}^{+}$and $\hat{a}_{k}$ are creation and annihilation operators of an electron with momentum $k$.

To complete such a description of an electronic system a number of important approximations was made. First, the concept of a Fermi liquid was implemented. Second, the BCS assumed the simplest form of the matrix element of electron-electron attraction

$$
\begin{aligned}
& V_{k, k^{\prime}}=-V=\text { const }<0, \text { if } \zeta_{k}, \zeta_{k^{\prime}}<\hbar \omega_{D}, \\
& V_{k, k^{\prime}}=0, \text { if } \zeta_{k}, \zeta_{k^{\prime}}>\hbar \omega_{D},
\end{aligned}
$$

where $\omega_{D}$ is Debye frequency, $\zeta_{k}=\frac{\hbar^{2} k^{2}}{2 m}-E_{F}$.

This hypothesis does not impose significant restrictions on the applicability of the theory. Indeed, the scale of the electron-phonon interaction is determined by the phonon energy $\hbar \omega_{D}$, which has an order of $10^{-2} \mathrm{eV}$ and much smaller than the Fermi energy in typical 
metals. Thus the theory should take into account only wave vectors $\mathrm{k}$ lying in the thin layer around Fermi sphere, where amplitudes of $\mathrm{k}$ are almost constant. This assumption justifies the choice of matrix element $V_{k, k^{\prime}}$ as a constant. In addition, the BCS theory requires zero orbital momentum of the Cooper pair $L=0$. This type of superconductivity is called swave [4]. The Pauli principle also allows the quantum state of the electrons pair with an even total orbital angular momentum, for example pair momentum $L=2$ corresponds to d-wave superconductivity [5-9]. The d-wave superconductivity occurs in the ceramic materials with strong anisotropy. Pairing with odd orbital momentum is also allowed but requires parallel spins of paired electrons. This type of superconductivity is called p-wave $[10,11,81]$ and probably exists in heavy metals compounds, like $S r_{2} R u O_{4}[13-18]$ or heavy fermion compounds [19-27].

The solution of the quantum mechanical problem $\hat{H} \psi=E \psi$ in the formalism of second quantization makes it possible to find the eigenfunctions of the electronic band in such a system and to determine the ground state. Superconductors have a property that the pair states are energetically more favorable than the single ones. As a result the wave function in ground state is sought in the form $|\psi\rangle=\prod_{k}\left(u_{k}+v_{k} \hat{a}_{k \uparrow}^{+} \hat{a}_{-k \downarrow}^{+}\right)|0\rangle$, where $u_{k}$ and $v_{k}$ are unknown coefficients. It should be noted that these factors have a clear physical meaning: $u_{k}$ is the probability amplitude of having the state with momenta $\mathrm{k},-\mathrm{k}$ and spins $\uparrow$ and $\downarrow$ unoccupied; while $v_{k}$ is the probability amplitude that its state is occupied. To calculate $u_{k}$ and $v_{k}$ one should minimize the thermodynamic potential $\langle\psi|H-\mu N| \psi\rangle$. This procedure leads to the following equation for pair potential $\Delta$

$$
\begin{aligned}
\Delta_{k}= & -\frac{1}{2} \sum_{l} V_{k, k^{\prime}} \frac{\Delta_{k^{\prime}}}{\sqrt{\zeta_{k^{\prime}}^{2}+\Delta_{k^{\prime}}^{2}}} ; \\
& v_{k}=\frac{1}{2}\left(1-\frac{\zeta_{k}}{\sqrt{\zeta_{k}^{2}+\Delta_{k}^{2}}}\right) \quad u_{k}=\frac{1}{2}\left(1+\frac{\zeta_{k}}{\sqrt{\zeta_{k}^{2}+\Delta_{k}^{2}}}\right) .
\end{aligned}
$$

Cooper's assumption $V_{k, k^{\prime}}=-V$ simplifies this problem to $\Delta=\frac{V}{2} \sum_{k^{\prime}} \frac{\Delta}{\sqrt{\zeta_{k^{\prime}}^{2}+\Delta^{2}}}$. There are two solutions of this equation, which are related to extrema of free energy. The first one with $\Delta=0$ and $v_{k}=\frac{1}{2}\left(1-\frac{\zeta_{k}}{\left|\zeta_{k}\right|}\right)$ corresponds to a trivial ground state. There is another minimum of free energy at $\Delta=\frac{\hbar \omega_{D}}{\sinh (1 / N(0) V)}$, where $N(0)$ is a density of states at zero energy. It means that the Fermi-Dirac distribution is broadened even at zero temperature (see Fig.0.1). Another important feature of BCS theory is the existence of an energy gap in electronic spectrum. The gap occurs since the excited quasiparticles should have energy larger than pair potential $\Delta$ to escape from ground state. 


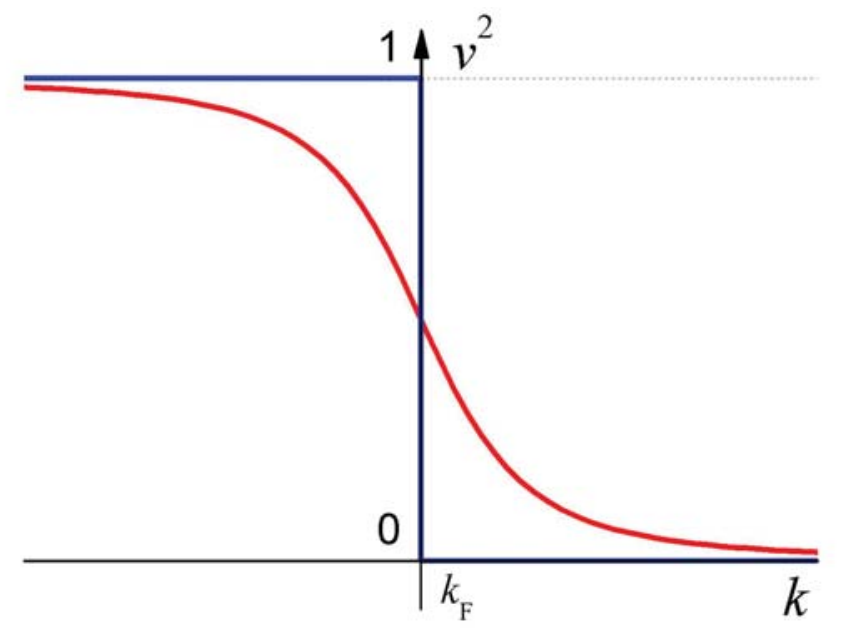

Figure 0.1. Occupation probability of electronic states $v^{2}$ versus wave-number $\mathrm{k}$ in the ground state for normal metal (blue) and superconductor (red)

Independently of the BCS, Bogolyubov had generalized theory for the spatially inhomogeneous case. He suggested the transformation, which changes operators of creation and annihilation of electrons to new composite operators. These operators describe effective quasiparticles. Applying the Bogolyubov transformations, it is possible to write stationary Schroedinger equation in the Bogolyubov-de Gennes form [28] for spinors $\left(\begin{array}{l}u_{k}(r) \\ v_{k}(r)\end{array}\right)$

$$
E_{K}\left(\begin{array}{l}
u_{k}(r) \\
v_{k}(r)
\end{array}\right)=\left(\begin{array}{cc}
\hat{H}_{e}+B(r) & \Delta(r) \\
\Delta(r) & -\left(\hat{H}_{e}^{*}+B(r)\right)
\end{array}\right)\left(\begin{array}{l}
u_{k}(r) \\
v_{k}(r)
\end{array}\right)
$$

with self-consistency equation

$$
\Delta(r)=\sum v_{k}^{*} u_{k} t h\left(\frac{E_{k}}{2 T}\right)
$$

where $H_{e}=\frac{1}{2 m}\left(-i \hbar \nabla-\frac{e}{c} \vec{A}\right)^{2}+U_{0}-E_{F}$ - single-particle Hamiltonian, $B(r)=-V<\psi_{r \uparrow}^{+} \psi_{r \uparrow}>$ - self consistent potential for electron-electron Coulomb interaction.

Bogolyubov-de Gennes equations allow to describe hybrid structures consisting of superconductors and normal metals. This approach has a significant drawback: every single inhomogeneity should be described with the potential barrier and therefore the solution of equations for a rather disordered material becomes extremely complicated. Thus, the theory of BdG can be applied in practise only for "clean" materials. "Clean" material implies that the electronic mean free path in it is much larger than the size of Cooper pairs, which is called the superconducting coherence length. 
The similar physics is included in Gorkov equations [29-31] which describe superconducting state in terms of Green functions $G\left(r, r_{1}\right)$ and $F\left(r, r_{1}\right)$. These functions have physical meaning similar to the coefficients $u_{k}$ and $v_{k}$. Usually these equations can be written in the Nambu space [32] in the following form:

$$
\begin{gathered}
\left(i \omega \sigma_{3}+\left(\hat{H}_{e}+B\right) \sigma_{0}+\hat{\Delta}\right) \hat{G}=\delta\left(r-r_{1}\right), \\
\hat{G}=\left(\begin{array}{cc}
G & F \\
F^{*} & -G
\end{array}\right), \quad \hat{\Delta}=\left(\begin{array}{cc}
0 & \Delta \\
-\Delta^{*} & 0
\end{array}\right),
\end{gathered}
$$

where $\omega=\pi T(2 n+1)$ is Matsubara frequency, which permits to describe system at the finite temperatures [33].

However, the functions $\mathrm{G}$ and $\mathrm{F}$ contain redundant information about fast oscillations on atomic scale $\hbar v_{F} / E_{F}$, which doesn't influence on measurable parameters of the structures. Thus, the next step will be the use of the quasiclassical approximation. The scale of spatial variation of Green functions $\hbar v_{F} / E_{F}$ is much smaller than the coherence length $\xi_{0}=\hbar v_{F} / \Delta$. Thus the Gorkov functions can be averaged over the coordinate and the resulting functions $g(r, \theta)$ depend only on center of mass of Cooper pair $r$ and the direction of their movement $\theta$. The resulting equation for Green function $\hat{g}$ was derived by Eilenberger [34]

$$
\begin{gathered}
{\left[\omega \sigma_{3}+\Delta-1 / \tau\langle\hat{g}\rangle, \hat{g}\right]+v_{F} \partial_{r} \hat{g}=0,} \\
\hat{g}=\left(\begin{array}{cc}
g & f \\
f^{*} & -g
\end{array}\right), \quad \hat{\Delta}=\left(\begin{array}{cc}
0 & \Delta \\
-\Delta^{*} & 0
\end{array}\right),
\end{gathered}
$$

where $\tau$ - scattering time, $\langle.$.$\rangle - denotes angle averaging and \left[f_{1}, f_{2}\right]$ - commutator. Selfconsistency condition on the pairing potential takes the form:

$$
\Delta \ln \left(\frac{T}{T_{C}}\right)-\pi T \sum_{\omega}\left(\frac{\Delta}{\omega}-\langle f\rangle\right)=0 .
$$

The description of disordered superconductors (the electronic mean free path $l_{e}$ is much smaller than the coherence length $\xi_{0}$ ) can be further simplified. In this case, strong disorder provides isotropization of Green functions with small parameter $l_{e} / \xi_{0}$ and one can formulate the so-called Usadel equations [35] in terms of angle-averged Green Functions $F(r)$ and $G(r)$ 


$$
\begin{aligned}
& i \hbar D \partial_{r}\left(\hat{G} \partial_{r} \hat{G}\right)-\left[\hat{\Delta}+\left(\omega_{n}+i H\right) \sigma_{3}, \hat{G}\right]=0, \\
& \hat{G}=\left(\begin{array}{cc}
G & F \\
F^{*} & -G
\end{array}\right), \quad \hat{\Delta}=\left(\begin{array}{cc}
0 & \Delta \\
-\Delta^{*} & 0
\end{array}\right),
\end{aligned}
$$

where $D=\frac{v_{F} l_{e}}{3}$ is the diffusion coefficient. The equations are also complemented with selfconsistency relation for $\Delta$

$$
\Delta \ln \left(\frac{T}{T_{C}}\right)-\pi T \sum_{\omega}\left(\frac{\Delta}{\omega}-F_{\omega}\right)=0 .
$$

In principle, there are two equations for $\mathrm{F}$ and $\mathrm{G}$, but in practice one of them can be excluded by normalization condition $F F^{*}+G^{2}=1$. Thus one can explain physical meaning of these averaged functions: $F(r)$ is the probability amplitude of paired electron and $G(r)$ is the probability amplitude of unpaired electron at a given energy.

\section{Superconductor-Ferromagnet Hybrids}

In general, the interaction between superconductive and ferromagnetic orders leads to new phenomena. In the presence of exchange field $H$ splitting of energy of particles with the same momentum but different spins occurs. Since Cooper pairs form from electrons with equal energies, their wavenumbers become different, namelly instead of $(k,-k)$ they become $(\mathrm{k}+\mathrm{q},-\mathrm{k}+\mathrm{q})$, wher $\mathrm{q}$ is total momentum of pair. Such transformation leads to a spatial modulation of the wave function of the singlet state of Cooper pairs $\Psi=\psi_{\uparrow} \psi_{\downarrow}^{*}+\psi_{\downarrow} \psi_{\uparrow}^{*} \propto$ $\cos (q x)[36,37]$. In the case of the bulk ferromagnet, averaging over the phase of oscillatory dependence completely eliminates all signatures of superconductivity. This phenomenon makes impossible the existence of a ferromagnetic s-wave superconductor. Nevertheless, superconductivity and ferromagnetic orders can coexist in the vicinity of $\mathrm{S} / \mathrm{F}$ interface (Superconductor/Ferromagnet). Penetration of Cooper pair from superconductor in nonsuperconductive material is called the proximity effect. In the case of a ferromagnet, the superconductive correlations exhibit decaying oscillatory dependence on the distance $x$ from SF-boundary $\Psi \propto \exp \left(-x / \xi_{F}\right)=\exp \left(-\left(1 / \xi_{F 1}\right) x\right) \exp \left(-i\left(1 / \xi_{F 2}\right) x\right)[38]$. Here $\xi_{F}$ is a complex coherence length in ferromagnetic material, giving approximatelly by the following 
expression, which is often used by experimentalists [39]:

$$
\xi_{F 1,2}=\sqrt{\frac{\hbar D}{\sqrt{\pi^{2} T^{2}+H^{2}} \pm \pi T}} .
$$

Here, $\xi_{F 1}$ is the decay length, while $2 \pi \xi_{F 2}$ is the wavelength of the oscillations. For comparison, for the normal material $(\mathrm{H}=0)$ coherence length is monothonic and described by expression

$$
\xi_{N}=\sqrt{\frac{\hbar D}{2 \pi T}}
$$

Another aspect of superconductor-ferromagnet proximity effect is the possibility of formation of triplet Cooper pairs. There are two types of triplet pairs. The first one is related to the pairs consting of the electrons with total spin projection equal to zero on the magnetization axis. This type of order is called "short-range triplet". The situation drastically changes in the case of magnetic inhomogeneities: for example, in the case of multiple non-collinear magnetic layers. The presence of non-collinear magnetizations in the system leads to precession of the spin and appearance of pairs with finite spin projection. Since such pairs aren't destroyed by exchange field, and this order parameter has characterestic decay length comparable with $\xi_{N}$ and is called "long-range triplet" component. [40-42]

\section{Motivation}

In recent years the development of superconducting electronics is rapidly growing field. Energy efficiency and high characteristic frequencies of supeconductive devices may potentially provide significant benefit compared to other proposals of future electronics circuits. The main direction of this field is the development of controllable superconducting devices and memory elements. The one of the possible ways to control properties of superconducting structures is the usage of ferromagnetic layers in Josephson junctions [43]- [70]. There are a lot of different proposals and concepts in this field.

It took a long time before the first experimental observation of coupling even through single ferromagnet layer [71]. This problem was solved by implementation of soft magnetic CuNi alloys. Shortly after, the experiments provided the evidence of $\pi$-junctions through phase-sensitive experiments [72] and demonstrated temperature induced crossover between 0 and $\pi$-states [73]. At the same time, other challenges appeared in the field.

One of the main problems of supeconductive electronics is the creation of $\varphi$-junction, 
the structure with nontrivial phase $\varphi$ in the ground state. Implementation of these structures in conventional schemes RSFQ-logic (Rapid Single Flux Quantum) can reduce the size of the curcuits and increase their speed. [74-76]. Another possibility is development of quantum bits using $\varphi$-contacts. It would mean downsizing, low inductance and decrease the sensitivity to external noise [77-79]. However, the development of $\varphi$-junction reveals the problem of miniaturization. Most of earlier proposals are addressed to complex structures. For example, the first $\varphi$-junction was predicted by Mints [80] for the case of randomly distributed alternating $0-$ and $\pi$ - Josephson junctions along grain boundaries in high $T_{c}$ cuprates with d-wave order parameter symmetry. It was shown later that $\varphi$-junctions can also be realized in the periodic array of 0 and $\pi$ SFS junctions [81, 82]. However, these structures are in the long Josephson junction regime $\left(W>\lambda_{J}\right)$ [83-85]. Thus the problem of designing small-size phi-junctions was still open at the time of starting this $\mathrm{PhD}$ project.

Another demand of superconductive electronics is development of high-speed superconductive memory element. Usually such devices include multiple ferromagnetic layers. [86-89] Typical spin valve device contains two magnetic layers $F_{1}$ and $F_{2}$ between superconductive electrodes. The layers $F_{1}$ and $F_{2}$ can change their mutual magnetization directions from parallel to antiparallel orientation. Switching to antiparallel orientation makes effective exchange field of weak link smaller and increases critical current $J_{C}$. However, this type of devices has some drawbacks. The first one is that switching process requires change of the magnetization in one $F_{1}$ layer only and remains constant in the $F_{2}$ layer, i.e. using two different materials, the soft magnet and the hard one. In particular geometries of junctions, this problem can be solved by using antiferromagnetic substrate in order to fix magnetization of an adjacent layer. The disadvantage of this solution is complexity of resulting structure which is difficult to fabricate. The second and the main drawback of spin-valve devices is relatively small product of critical current and normal resistance $J_{C} R_{N}$ which determines characteristic frequency of the junction. In this case performance of memory elements is much worse compared to tunnel SIS junctions usually used in basics RSFQ circuits: junctions of SFFS type have small normal resistance $R_{N}$ due to lack of the tunnel barrier, and SIFFS type junctions have large normal resistance $R_{N}$, but their critical current $J_{C}$ is much smaller.

There are some concepts of long-range triplet devices for memory applications [9092]. They include multiple magnetic layers with non-collinear magnitezation in the area of the weak link. As a result supercurrent is almost insensitive to suppression in ferromagnetic 
layers. However inherent drawback of this aprroach is the presence of too many interfaces [93] leading suppression of supercurrent.

The alternative approach to make fast magnetic device is to include an additional superconductive layer inside the weak link, which can support superconductivity. This approach was proposed in [94] and it permits to realize high $J_{C} R_{N}$ in experiment. However at the moment of starting this $\mathrm{PhD}$ project this problem wasn't solved theoretically. Therefore this question is one of the goals of this thesis.

Josephson devices based on unconventional superconductors are also very promising for practical applications. D-wave and p-wave superconductors have special properties which can be implemented in electronic devices. For example, Josephson junctions based on d-wave superconductive electrodes with faceted interface can provide $\varphi$-state. [95] Implementation of topological materials is also promising, since the promixity effect between superconductor and topological insulator may provide realization of Majorana state [96], which which was suggested as possible realisation of noise-protected quantum systems [97].

P-wave superconductors provide interesting model system to study such effects experimentally, since they have intrinsic long-range triplet superconductivity and exhibit midgap Andreev bound state in the vicinity of interfaces. Therefore in this work we have also studied the problem of surface superconductivity in triplet $p$-wave materials.

To summarize, the purpose of this work is theoretical study of the proximity effect in hybrid structures, involving s- and p - wave superconductors and ferromagnets. This study provides new concepts of controllable superconducting devices. Finally, all these statements are merged into discussion about proximity effect between different superconductive and normal materials, and their implementation in the some controllable devices and structures.

\section{Contents of Chapters}

In Chapter 1 the $\varphi$-junctions are discussed. These junctions have nontrivial Josephson phase $\varphi$ (different from $\pi n$ ) in the ground state. Various geometries of junctions are considered and their properties are compared.

In section 1.2 we formulate theory of generation of the second harmonic in current phase relation in Josephson junctions. We describe the relations between high order harmonics and multiple Andreev reflections in the weak link and describe properties of current-phase relation. 
In section 1.3 we formulate the model for the description of Josephson $\varphi$-junctions in the frame of Usadel equations. We numerically solve two dimensional nonlinear problem in assumption that all materials are in dirty limit and pairing constant inside weak link is equal to zero. This model allows to calculate the distribution of Josephson current across the structure.

In section 1.4 the S-FN-S junction is suggested to realize $\varphi$-junction. Such geometry includes two bulk superconducting electrodes. We show that the presence of two different channels of current transport (normal and ferromagnetic) permits the realization of $\varphi$-state at the point of $0-\pi$ transition.

Section 1.5 is devoted to overlap geometry of $\varphi$-junctions, with superconductor electrodes placed on the NF bilayer. We show that the ordering of normal and ferromagnet layers is important in this case. If the $\mathrm{N}$ layer is on the top, then it forms main current channel between electrodes and $\varphi$-state is prohibited. Contrary to this, in the case when F-layer is on the top, there are two competing current channels which form $\varphi$-state.

In section 1.6 we consider ramp-overlap type structure, with SFS junction placed on the top of a normal substrate. We conclude that this geometry provides a broad area of existence of $\varphi$-state and it can be used as practical way to create submicron scale $\varphi$-junction.

In Chapter 2 we consider SIsFS junction. This structure includes two bulk superconducting electrodes $\mathrm{S}$ and complex interlayer consisting of tunnel barrier "I", ferromagnetic film "F" and thin superconductive spacer "s" between them. We demonstrate that SIsFS junctions have several distinct regimes of supercurrent transport and we examine spatial distributions of the pair potential across the structure in different regimes. We study the crossover between these regimes which is caused by shifting the location of a weak link from the tunnel barrier 'I' to the F-layer.

In section 2.2 the model of this structure is formulated. We use the same formalism of Usadel equations as introduced in the previous chapter. However, we make particular attention to the solution the self-consistency equation for superconducting pair potential, since the state of the s-layer is crucially important for current-carrying state in this structure.

Section 2.3 is devoted to analytical calculations in the limit of high temperature. In this regime self-consistent solution in the thin middle superconductive film can be considered in the frame of Ginzburg-Landau equations. We show that strong deviations of the CPR from sinusoidal shape occur even in a vicinity of $T_{C}$, and these deviations are strongest in 
the vicinity of $0-\pi$ crossover.

In section 2.4 is devoted to numerical calculations at arbitrary temperatures. We demonstrate the existence of temperature-induced crossover between 0 and $\pi$ states in the contact and show that smoothness of this transition strongly depends on the CPR shape.

In Chapter 3 we compare the theoretical model for SIsFS junctions with experimental data obtained by HYPRES (USA) and ISSP RAS (Chernogolovka, Russia) groups.

In section 3.2 we discuss modes of operation of SIsFS junction and consider temperature-induced switching between them. We compare the results of our calculations with experimental data provided by Chernogolovka group.

Section 3.3 is devoted to behaviour of SIsFS junction in external magnetetic field. We fit the experimental hysteretical dependence $I_{C}(H)$ and discuss properties of magnetic interlayers, which can provide such dependence.

In Chapter 4 the problem of surface superconductivity in triplet $p$-wave superconductors is considered. In the $p_{x}$ case we demonstrate the robustness of the zero-energy peak in the density of states (DoS) with respect to surface roughness, in contrast to the suppression of such a peak in the case of $d_{x y}$ symmetry. This effect is due to stability of odd-frequency pairing state at the surface with respect to disorder. In the case of the chiral $p_{x}+i p_{y}$ state we demonstrate the appearance of a complex multi-peak subgap structure in the spectrum with increasing surface roughness.

In section 4.1 two-dimensional model for triplet $p$-wave superconductors is formulated. We derive boundary conditions for Eilenberger equations at rough interfaces and develop the approach for self-consistent solution for the spatial dependence of $p_{x}$ and $p_{x}+i p_{y}$ -wave pair potentials.

In section 4.2 the pair potential in the vicinity of boundary is discussed. We demonstrate the difference between $p_{x}$ and $p_{y}$ parts of order parameter and demonstrate their spatial dependencies as a function of boundary roughness.

In section 4.3 the pair amplitudes and symmetry relations are discussed for $p_{x}$ and $p_{x}+i p_{y}$-wave superconductors.

Section 4.4 is devoted to calculatiion of density of states at the surface. We demonstrate the existence of of a complex multi-peak subgap structure in the spectrum with increasing surface roughness. Furthermore, we show that the subgap peak related to Andreev bound state is stable with respect to roughness. 


\section{Bibliography}

[1] H. Froehlich, Proc. Roy. Soc., A215, 291 (1952)

[2] L.N. Cooper, Phys. Rev. 104 , 1189 (1956)

[3] J. Bardeen, L. N. Cooper, J. R. Schrieffer, Phys. Rev. 106, 162 (1957)

[4] V. V. Schmidt, "The physics of superconductors: introduction to fundamentals and applications", Springer (1997)

[5] Yu. S. Barash, A. A. Svidzinsky, and H. Burkhardt, Phys. Rev. B 55, 15282 (1997)

[6] M. Fogelstrom, D. Rainer, and J. A. Sauls, Phys. Rev. Lett. 79, 281 (1997)

[7] A. A. Golubov and M. Yu. Kupriyanov, Pis'ma Zh. Eksp. Teor. Fiz. 67, 478 ( 1998) [JETP Lett 67, 501 (1998)]

[8] A. A. Golubov and M. Yu. Kupriyanov, Superlattices and Microstructures, 25, 949 (1999)

[9] A. A. Golubov and M. Yu. Kupriyanov, Pis'ma Zh. Eksp. Teor. Fiz. 69, 242 ( 1999) [JETP Lett. 69, 262 (1999)]

[10] F. S. Bergeret, A. F. Volkov, K. B. Efetov, Rev. Mod. Phys. 77, 1321 (2005)

[11] K. B. Efetov, I. A. Garifullin, A. F. Volkov and K. Westerhilt, cond-mat/0610708 (2006)

[12] A. I. Buzdin, Rev. Mod. Phys. 77, 935 (2005)

[13] Y. Maeno, H. Hashimoto, K. Yoshida, S. Nishizaki, T. Fujita, J. G. Bednorz, and F. Lichtenberg, Nature (London) 372, 532 (1994)

[14] K. Ishida, H. Mukuda, Y. Kitaoka, K. Asayama, Z. Q.Mao, Y. Mori, and Y. Maeno, Nature (London) 396, 658 (1998) 
[15] G. M. Luke, Y. Fudamoto, K. M. Kojima, M. I. Larkin, J.Merrin, B. Nachumi, Y. J. Uemura, Y. Maeno, Z. Q. Mao, Y. Mori, H. Nakamura, and M. Sigrist, Nature (London) 394, 558 (1998)

[16] A. P. Mackenzie and Y. Maeno, Rev. Mod. Phys. 75, 657 (2003)

[17] K. D. Nelson, Z. Q. Mao, Y. Maeno and Y. Liu, Science 306, 1151 (2004)

[18] Y. Asano, Y. Tanaka, M. Sigrist and S. Kashiwaya, Phys.Rev. B 67, 184505 (2003); Phys. Rev. B 71, 214501 (2005)

[19] H. Tou, Y. Kitaoka, K. Ishida, K. Asayama, N. Kimura, Y. Onuki, E. Yamamoto, Y. Haga and K. Maezawa, Phys.Rev. Lett. 80, 3129 (1998)

[20] V. Muller, Ch. Roth, D. Maurer, E. W. Scheidt, K. Lers, E.Bucher and H. E. Bmel, Phys. Rev. Lett. 58, 1224 (1987)

[21] Y. J. Qian, M. F. Xu, A. Schenstrom, H. P. Baum, J. B.Ketterson, D. Hinks, M. Levy and B. K. Sarma, Solid State Commun., 63, 599 (1987)

[22] A. A. Abrikosov, J. of Low Temp. Phys. 53, 359 (1983)

[23] H. Fukuyama and Y. Hasegawa, J. Phys. Soc. Jpn. 56, 877 (1987)

[24] A. G. Lebed, K. Machida and M. Ozaki, Phys. Rev. B 62, 795 (2000)

[25] S. S. Saxena, P. Agarwal, K. Ahilan, F. M. Grosche, R.K. W. Haselwimmer, M. J. Steiner, E. Pugh, I. R. Walker, S. R. Julian, P. Monthoux, G. G. Lonzarich, A. Huxley, I.Shelkin, D. Braithwaite, and J. Flouquet, Nature (London) 406, 587 (2000)

[26] C. Peiderer, M. Uhlarz, S. M. Hayden, R. Vollmer, H. v.Lohneysen, N. R. Bernhoeft, and G. G. Lonzarich, Nature (London) 412, 58 (2001)

[27] D. Aoki, A. Huxley, E. Ressouche, D. Braithwaite, J. Flouquet, J. Brison, E. Lhotel, and C. Paulsen, Nature (London) 413, 613 (2001)

[28] P. G. De Gennes, "Superconductivity of Metals and Alloys (Advanced Book Classics)", Addison-Wesley Publ. Company Inc (1999)

[29] L. P. Gor'kov , Zh. Eksp. Teor. Fiz. 34, 735 (1958) 
[30] A.V. Svidzinskii, "Spatially inhomogeneous problems in the theory of superconductivity. Methods of quantum field theory in statistical physics", Nauka (1982)

[31] A. A. Abrikosov, , L. P. Gor'kov, and I. E. Dzyaloshinskii, Zh. Eksp. Teor. Fiz. 36 (1959)

[32] Y. Nambu, Phys. Rev. 117, 648 (1960)

[33] T. Matsubara, Prog. Theor. Phys. 114, 4, 351 (1955)

[34] G. Eilenberger, Z. Phys. 214, 196, (1968)

[35] K. D. Usadel, Phys. Rev. Lett. 25, 507, (1970)

[36] P. Fulde, R.A. Ferrell. Phys. Rev. 135, A550 (1964).

[37] A.I. Larkin, Yn.N. Ovchinnikov. Zh. Exp. Teor. Fiz 47, 1136 (1964)[Sov. Phys. JETP 20, $762(1965)]$.

[38] E. A. Demler, G.B. Arnold and M.R. Beasley, Phys. Rev. B, 55, 22, 15174 (1997)

[39] V.A. Oboznov, V.V. Bol'ginov, A.K. Feofanov, V.V. Ryazanov, A.I. Buzdin , Phys. Rev. Lett., 96, 197003 (2006)

[40] A. F. Volkov, F. S. Bergeret, and K. B. Efetov, Phys. Rev. Lett. 90, 11, 117006 (2003)

[41] F. S. Bergeret, A. F. Volkov, and K. B. Efetov, Phys. Rev. B 64, 134506 (2001)

[42] F. S. Bergeret, A. F. Volkov, and K. B. Efetov, Phys. Rev. B 68, 064513 (2003)

[43] A. A. Golubov, M. Yu. Kupriyanov, and E. Il'ichev, Rev. Mod. Phys. 76, 411 (2004)

[44] A.I. Buzdin, M.Yu. Kupriyanov, Pis'ma Zh. Exp. Teor. Fiz, 52, 1089 (1990)[JETP Lett. 52, 487 (1990)]

[45] Z. Radovic, M. Ledvij, Lj. Dobroslaljevic-Grujic, A.I. Buzdin, J.R. Clem, Phys. Rev. B, 44, 759 (1991)

[46] L.R. Tagirov, Physica C 307, 145 (1998)

[47] M.G. Khusainov and Yu.N. Proshin, Phys. Rev. B, 56, R14283 (1997)

[48] L.N. Bulaevskii, V.V. Kuzii, A.A. Sobyanin, Pis'ma Zh. Exp. Teor. Fiz. 25, 314 (1977)[JETP Lett. 25, 290 (1977)] 
[49] A.I. Buzdin, M.Yu. Kupriyanov, Pis'ma Zh. Exp. Teor. Fiz. 53, 308 (1991)

[50] T. Kontos, M. Aprili, J. Lesueur, F. Genet, B. Stephanidis, and R. Boursier, Phys. Rev. Lett., 89, 13, 137007-1 (2002)

[51] T. Kontos, M. Aprili, J. Lesueur, X. Grison. Phys. Rev. Lett., 86, 2, 304, (2001)

[52] J. Rammer, H. Smith, Rev. Mod. Phys. 58, 2, 323 (1986)

[53] W. Belzig, F. K. Wilhelm, C. Bruder, G. Schon, A. D. Zaikin, 25, 5/6 (1999)

[54] M. Yu. Kuprianov and V. F. Lukichev, Zh. Eksp. Teor. Fiz. 94, 139 (1988) [Sov. Phys. JETP 67, 1163 (1988)]

[55] C. Bell, R. Loloee, G. Burnell, and M. G. Blamire, Phys. Rev. B 71, 180501 (R) (2005)

[56] V. Shelukhin, A. Tsukernik, M. Karpovski, Y. Blum, K. B. Efetov, A. F. Volkov, T. Champel, M. Eschrig, T. Lofwander, G. Schon, and A. Palevski, Phys. Rev. B, 73 174506 (2006)

[57] M. Weides, K. Tillmann, and H. Kohlstedt, Physica C 437-438, 349-352 (2006)

[58] M. Weides, M. Kemmler, E. Goldobin, H. Kohlstedt, R. Waser, D. Koelle, R. Kleiner, Phys. Rev. Lett., 97, 247001 (2006)

[59] H. Sellier, C. Baraduc, F. Lefloch, and R. Calemczuck, Phys. Rev. Lett. 92, 25, 257005 (2004)

[60] F. Born, M. Siegel, E. K. Hollmann, H. Braak, A. A. Golubov, D. Yu. Gusakova, and M. Yu. Kupriyanov, Phys. Rev. B. 74, 140501 (2006)

[61] L. B. Ioffe, V. B. Geshkenbein, M. V. Feigelman, A. L. Fauchere, G. Blatter, Nature (London) 398, 679 (1999)

[62] A. V. Ustinov, V. K. Kaplunenko, J. Appl. Phys. 94, 5405 (2003)

[63] G. Blatter, V. B. Geshkenbein, L.B. Ioffe, Phys. Rev. B. 63, 174511 (2001)

[64] E. Terzioglu, M.R. Beasley. IEEE Trans. Appl. Superc. Phys. Rev. B. 8, 48 (1998)

[65] Hans Hilgenkamp, M.R. Supercond. Sci. Technol. 21, 034011, (2008)

[66] G. Binasch, P. Grunberg, F. Saurenbach and W. Zinn, Phys. Rev. B 39, 4828 (1989) 
[67] F. S. Bergeret, A. F. Volkov, and K. B. Efetov, Phys. Rev. Lett. 86, n. 14, 3140 (2001)

[68] Ya. M. Blanter and F. W. J. Hekking, Phys. Rev. B 69, 024525 (2004)

[69] A. A. Golubov, M. Yu. Kupriyanov, and Ya. V. Fominov, Pis'ma Zh. Eksp. Teor. Fiz., 75, 223 (2002) [JETP Letters, 75, 190 (2002).

[70] I. B. Sperstad, J. Linder and A. Sodbo, Phys. Rev. B 78, 104509 (2008).

[71] V. V. Ryazanov, V. A. Oboznov, A. V. Veretennikov, A. Yu. Rusanov, Phys. Rev. B 65, 02051(R) (2001).

[72] S. M. Frolov, D. J. Van Harlingen, V. A. Oboznov, V. V. Bolginov, V. V. Ryazanov, PhysRevB. 70, 144505, (2004)

[73] V. V. Ryazanov, V. A. Oboznov, A. Yu. Rusanov , A.V. Veretennikov, A.A. Golubov , J. Aarts, Phys. Rev. Letters, 86, 11, 2427, (2001)

[74] P. Bunyk, K. Likharev, and D. Zinoviev, Int. Journal of High Speed Electronics and Systems, 11, 1, 257, (2001)

[75] A. V. Ustinov , V. K. Kaplunenko, J. Appl. Phys., 94, 5405, (2003)

[76] O. Wetzstein , T. Ortlepp, R. Stolz, J. Kunert , H.-G. Meyer, H. Toepfer, IEEE Transactions on Applied Superconductivity, 21, 814, (2011)

[77] C. H. Van der Waal, A. C. J. ter Haar, F. K. Wilhelm, R. N. Schouten, C. J. P. M. Harmans, T. P. Orlando, Seth Lloyd, J. E. Mooij, Science, 290, 773, (2000)

[78] M. H. S. Amin , A. Yu. Smirnov, A. M. Zagoskin , T. Lindstrom , S. A. Charlebois , T. Claeson, A. Ya. Tzalenchuk, Phys. Rev. B, 73, 064516-1-5, (2005)

[79] N. V. Klenov, V. K. Kornev , N. F. Pedersen, Physica C, 435, 114 (2006)

[80] R.G. Mints, Phys. Rev. B 57, R3221, (1998)

[81] A. Buzdin and A. E. Koshelev, Phys. Rev. B 67, 220504(R), (2003)

[82] N. G. Pugach, E. Goldobin, R. Kleiner, and D. Koelle, Phys. Rev. B., 81, 104513, (2010) 
[83] H. Sickinger, A. Lipman, M. Weides, R. G. Mints, H. Kohlstedt, D. Koelle, R. Kleiner, E. Goldobin,Physical review letters 109 (10), 107002, (2012)

[84] E. Goldobin, H. Sickinger, M. Weides, N. Ruppelt, H. Kohlstedt, R. Kleiner, D. Koelle, Applied Physics Letters, 102 (24), 242602, (2013)

[85] A. Lipman, R.G. Mints, R. Kleiner, D. Koelle, E. Goldobin, Physical Review B, 90 (18), 184502, (2014)

[86] B. Baek, W. H. Rippard, S. P. Benz, S. E. Russek, P. D. Dresselhaus, Nature Comm., $5,(2014)$

[87] F. S. Bergeret, A. F. Volkov, and K. B. Efetov, Phys. Rev. Lett., 86, 3140 (2001).

[88] V. N. Krivoruchko and E. A. Koshina, Phys. Rev. B, 63, 224515 (2001); 64, 172511 (2001).

[89] A. A. Golubov, M. Yu. Kupriyanov, and Ya. V. Fominov, JETP Letters, 75, 190, (2002).

[90] Y. V. Fominov, A. A. Golubov, T. Yu. Karminskaya, M. Yu. Kupriyanov, R. G. Deminov, L. R Tagirov, JETP Lett. 91, 308, (2010).

[91] M. Houzet and A. I. Buzdin, Phys. Rev. B, 76, 060504 R, (2007)

[92] C. Richard, M. Houzet, J. S. Meyer, Phys. Rev. Lett., 110 (21), 217004, (2013)

[93] B. Niedzielski, S. Diesch, E. Gingrich, Y. Wang, R. Loloee, W. Pratt, N. Birge, IEEE Tran. on Appl. Supercond. 24, 4 (2014)

[94] T. I. Larkin, V. V. Bol'ginov, V. S. Stolyarov, V. V. Ryazanov, I. V. Vernik, S. K. Tolpygo, and O. A. Mukhanov, Appl. Phys. Lett. 100, 222601, (2012).

[95] H. Hilgenkamp, H. J. H. S. Ariando, D. H. Blank, G. Rijnders, H. Rogalla, J. R. Kirtley, C. C. Tsuei, Nature, 422 (6927), 50-53, (2003)

[96] M. Z. Hasan, C. L.Kane, Reviews of Modern Physics, 82 (4), 3045, (2010)

[97] A. Y. Kitaev,Annals of Physics, 303 (1), 2-30, (2003) 


\section{Chapter 1}

\section{Josephson $\varphi$-junctions based on structures with complex normal/ferromagnet bilayer}

\section{$1.1 \quad$ Introduction}

The relation between supercurrent $I_{S}$ across a Josephson junction and phase difference $\varphi$ between the phases of the order parameters of superconducting (S) banks is an important characteristic of a Josephson structure [1,20]. In standard SIS structures with tunnel type of conductivity of a weak link, the current-phase relation (CPR) has the sinusoidal form $I_{s}(\varphi)=A \sin (\varphi)$. On the other hand, in SNS or SINIS junctions with metallic type of conductivity the smaller the temperature $T$ the larger the deviations from the $\sin (\varphi)$ form [1] and $I_{S}(\varphi)$ achieves its maximum at $\pi / 2 \leq \varphi \leq \pi$. In SIS junctions the amplitude $B$ of second harmonic in $\mathrm{CPR}, B \sin (2 \varphi)$, is of the second order in transmission coefficient of the tunnel barrier I and therefore is negligibly small for all $T$. In SNS structures the second CPR harmonic is also small in the vicinity of critical temperature $T_{C}$ of superconductors, where $A \sim\left(T_{C}-T\right)$. At low temperatures $T \ll T_{C}$, the coefficients $A$ and $B$ have comparable magnitudes, thus giving rise to qualitative modifications of CPR shape with decrease of $T$.

It is important to note that in all types of junctions discussed above the ground state is achieved at $\varphi=0$, since at $\varphi=\pi$ a junction is at nonequilibrium state.

The situation changes in Josephson structures involving ferromagnets as weak link materials. The possibility of the so-called $\pi$-state in SFS Josephson junctions (characterized by the negative sign of the critical current $I_{C}$ ) was predicted theoretically and observed experimentally [ [20]- [29]]. Contrary to traditional Josephson structures, in SFS devices it is possible to have the ground state $\varphi_{g}=\pi$ (so-called $\pi$-junctions), while the $\varphi=0$ corresponds to an unstable situation. It was proven experimentally $[13,15]$ that $\pi$-junctions can be used as on-chip $\pi$-phase shifters or $\pi$-batteries for self-biasing various electronic quantum and classical circuits. It was proposed to use self $\pi$-biasing to decouple quantum circuits from environment or to replace conventional inductance and strongly reduce the size of an elementary cell [16]. 
In some classical and quantum Josephson circuits it is even more interesting to create on-chip $\varphi$-batteries. They are $\varphi$-junctions, the structures having phase difference $\varphi_{g}=\varphi,(0<|\varphi|<\pi)$ between superconducting electrodes in the ground state. The $\varphi$ states were first predicted by Mints [33] for the case of randomly distributed alternating 0 - and $\pi$ - Josephson junctions along grain boundaries in high $T_{c}$ cuprates with d-wave order parameter symmetry. It was shown later that $\varphi$-junctions can be also realized in the periodic array of 0 and $\pi$ SFS junctions $[17,19]$. It was demonstrated that depending on the length of 0 or $\pi$ segments in the array, a modulated state with the average phase difference $\varphi_{g}$ can be generated if the mismatch length between the segments is small. This $\varphi_{g}$ can take any value within the interval $-\pi \leq \varphi_{g} \leq \pi$. Despite strong constraints on parameter spread of individual segments estimated in [36], remarkable progress was recently achieved on realization of $\varphi$-junctions in such arrays [37].

In general, in order to implement a $\varphi$-junction one has use a Josephson junction having non-sinusoidal current-phase relation, which, at least, can be described by a sum of two terms

$$
I_{S}(\varphi)=A \sin (\varphi)+B \sin (2 \varphi)
$$

Moreover, the following special relationship between the amplitudes of the CPR harmonics, $A$, and, $B$, is needed for existence of equilibrium stable state $[38,39]$

$$
|B|>|A| / 2, B<0
$$

In conventional junctions, the magnitude of $A$ is larger than that of $B$ and the inequalities (1.2) are difficult to fulfill. However, in SFS junctions in the vicinity of 0 to $\pi$ transition the amplitude of first harmonic in CPR is close to zero, thus opening an opportunity for making a $\varphi$ - battery, if $B$ can be made negative. It is well-known that SFS junctions with metallic type of conductivity, as well as SIFS structures [24,34] with high transparencies of SF interfaces have complex decay length of superconducting correlations induced into F-layer $\xi_{H}=\xi_{1}+i \xi_{2}$. Unfortunately, the conditions (1.2) are violated in these types junctions since the $A \sim \exp \left\{-L / \xi_{1}\right\} \cos \left(L / \xi_{2}\right), B \sim-\exp \left\{-2 L / \xi_{1}\right\} \cos \left(2 L / \xi_{2}\right)$, and for $L=(\pi / 2) \xi_{2}$ corresponding to the first $0-\pi$ transition the second harmonic amplitude $B$ is positive.

Quantitative calculations made in the framework of microscopic theory $[42,43]$ confirm the above qualitative analysis. In Ref. $[42,43]$ it was demonstrated that in SFS sand- 
wiches with either clean or dirty ferromagnetic metal interlayer the transition from 0 to $\pi$ state is of the first order, that is $B>0$ at any transition point [21].

It was suggested recently in [47]- [49] to fabricate the "current in plane" SFS devices having the weak link region consisting from NF or FNF multilayers with the supercurrent flowing parallel to FN interfaces. In these structures, superconductivity is induced from the S banks into the normal (N) film, while F films serves as a source of spin polarized electrons, which diffuse from $\mathrm{F}$ to $\mathrm{N}$ layer thus providing an effective exchange field in a weak link. Its strength it can be controlled [50,71] by transparencies of NF interfaces, as well as by the products of densities of states at the Fermi level, $N_{F}, N_{N}$, and film thicknesses, $d_{F}, d_{N}$ . It was shown in $[47]^{-}[48]$ that the reduction of effective exchange energy in a weak link permits to increase the decay length from the scale of the order of $\sim 1 \mathrm{~nm}$ up to $\sim 100$ $\mathrm{nm}$. The calculations performed in these papers did not go beyond linear approximation in which the amplitude of the second harmonic in the CPR is small. Therefore, the question of the feasibility of $\varphi$-contacts in these structures has not been studied and remains open to date.

The purpose of this paper is to demonstrate that the same "current in plane" devices (see Fig. 1.1) can be used as effective $\varphi$-shifters. The structure of the paper is the following. In Sec.1.2 we present general qualitative discussion of the microscopic mechanisms leading to formation of higher harmonics in the CPR. In Sec.2.2 we formulate quantitative approach in terms of Usadel equations. In Sec 1.4 the criteria of $\varphi$-state existence are derived for ramp-type S-FN-S structure. Section 1.6 shows the advantage of the other geometries in order to realize $\varphi$-state. Finally in Sec.1.7 we consider properties of real materials and estimate the possibility to realize $\varphi$-states using up-to-date technology.

\subsection{CPR formation mechanisms}

In this section we shall discuss microscopic processes which contribute to formation of CPR in Josephson junctions. The physical reason leading to the sign reversal of the coefficient $B$ in SFS junctions compared to that in SNS structures can be understood from simple diagram shown in Fig.1.2 illustrating the mechanisms of supercurrent transfer in double barrier Josephson junctions.

Consider electron-like quasiparticle $e^{-}$propagating across SINIS structure towards the right electrode. This quasiparticle can be reflected either in the Andreev or in the 


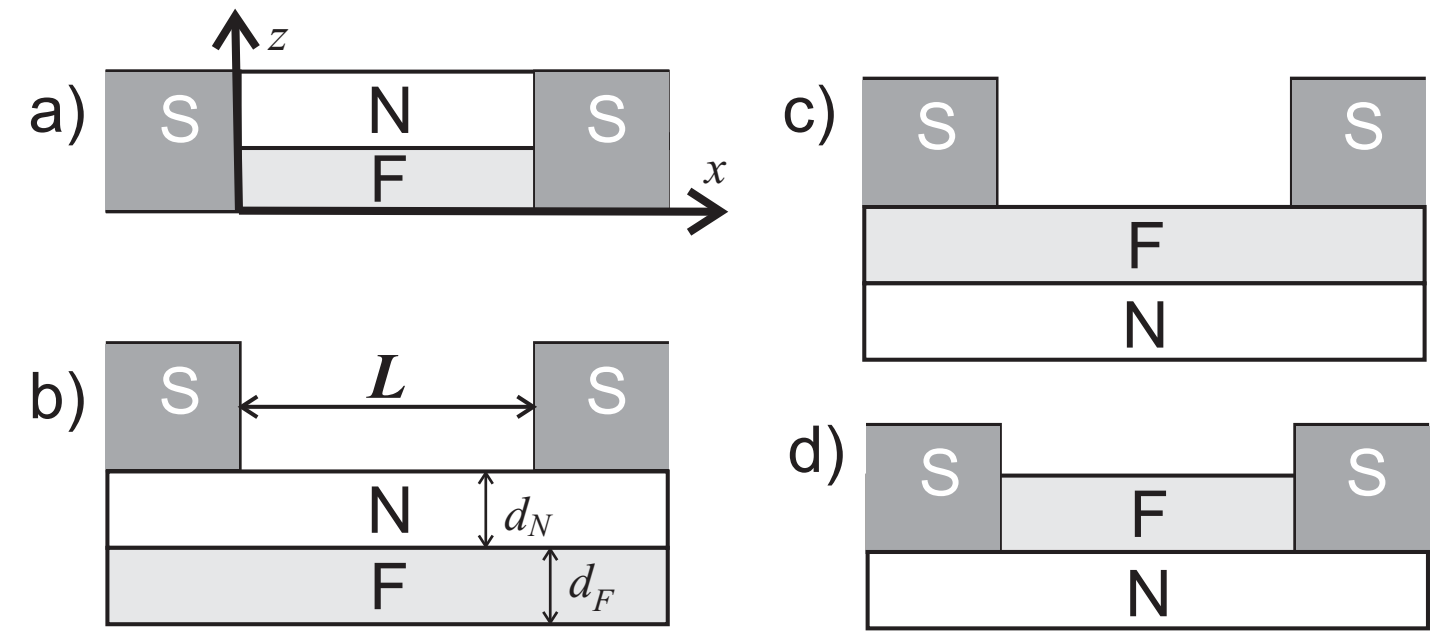

Figure 1.1. a) The $S-N F-S$ junction, b) the $S N F-N F-F N S$ junction, c) the $S F N-F N-N F S$ junction d) the $S N-F N-N S$ junction.

normal channel.

The result of the first process (see Fig.1.2a) is generation in the weak link region (with an amplitude proportional to $\exp \left(i \chi_{2}\right)$ ) of the hole $h^{+}$propagating in the opposite direction. Andreev reflection of this hole at the second interface (with an amplitude proportional to $\left.\exp \left(-i \chi_{1}\right)\right)$ results in transfer of a Cooper pair from the left to the right electrode with the rate proportional to the net coefficient of Andreev reflection processes [53,75] at both SN interfaces, $A R(\varphi)=\alpha(\varphi) \exp (i \varphi), \varphi=\left(\chi_{2}-\chi_{1}\right)$. The amplitude, $\alpha(\varphi)$, depends on geometry of a structure and on material parameters. Note that for given values of these parameters $\alpha(\varphi)=\alpha(-\varphi)$, according to the detailed balance relations [75]. Similar considerations show that a quasiparticle $e^{-}$moving towards the left electrode generates a Cooper pair propagating from the right to the left interface with the rate proportional to $A R(-\varphi)=\alpha(\varphi) \exp (-i \varphi)$. The difference between two processes described above determines a supercurrent $I_{S}$, which is proportional to $\sin (\varphi)$.

The result of the second process is the change (with an amplitude proportional to $\left.\exp \left(i \chi_{2}\right)\right)$ of the $e^{-}$propagation direction to the left electrode and nucleation of a Cooper pair and a hole propagating to the right electrode (with an amplitude proportional to $\exp (-i \varphi))$. After normal reflection from the right interface (with an amplitude proportional to $\left.\exp \left(i \chi_{2}\right)\right)$ the hole arrives at the left SN interface and closes this Andreev loop by generating a Cooper pair in the left electrode and an electronic state (with an amplitude proportional to $\left.\exp \left(-i \chi_{1}\right)\right)$. The Cooper pair have to undergo a full reflection at SN interface, thus again a pair is generated moving in the direction opposite to that in the main Andreev loop. The net coefficient of this Andreev reflection process is $B R(\varphi)=\beta(\varphi) \exp (2 i \varphi)$. For a 

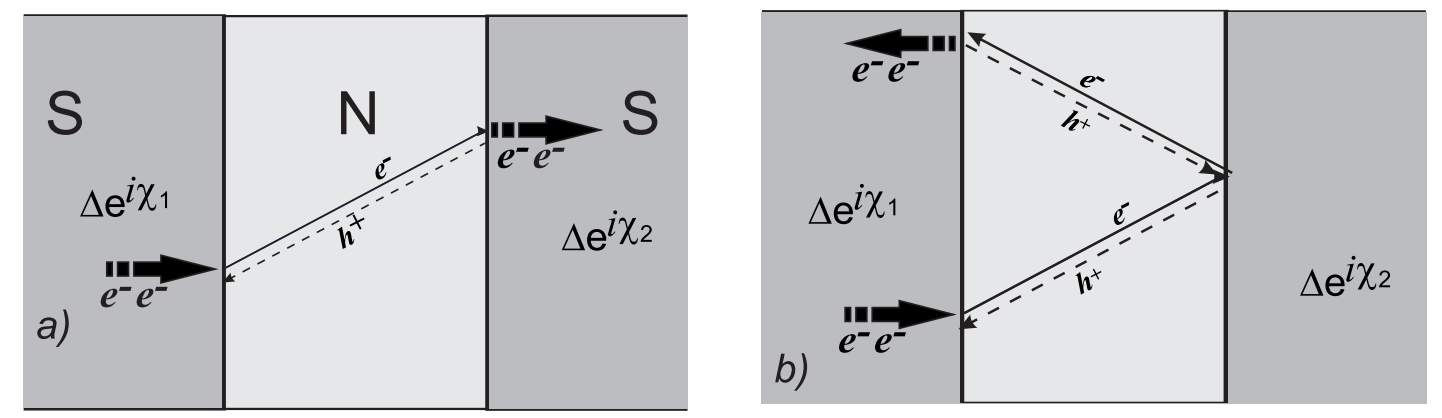

Figure 1.2. Diagrams of the processes forming the first (a) and second (b) harmonics of the CPR in the SNS and SFS structures.

quasiparticle $e^{-}$moving in the weak link towards the left electrode the same consideration leads to generation of two Cooper pairs moving from the left to the right with the rate proportional to $B R(-\varphi)=\beta(\varphi) \exp (-2 i \varphi)$. The difference between these two processes determines a part of supercurrent $I_{S}$ proportional to $\sin (2 \varphi)$.

We have shown that supercurrent components proportional to $\sin (\varphi)$ and $\sin (2 \varphi)$ have opposite signs, and the coefficient $B$ in Eq.(2.4) is negative. This statement is in a full agreement with calculations of the CPR performed in the frame of microscopic theory of superconductivity $[1,20]$. It is valid if a supercurrent across a junction does not suppress superconductivity in S electrodes in the vicinity of SN interfaces [37-39]. In addition, an effective path of the particles in the second process discussed above is two times larger than in the first one. This leads to stronger decay of the second harmonic amplitude $B$ with increasing the distance $L$.

In SFS junctions the situation becomes more complicated. The exchange field, $H$, in the weak link removes the spin degeneracy of quasiparticles. As a result, one has to consider four types of Andreev's loops instead of two loops discussed above. One should also take into account the fact that wave function of a quasiparticle propagating through the weak link acquires an additional phase shift $\varphi_{H}$ proportional to the magnitude of the exchange field [57]. The sign of $\varphi_{H}$ depends on mutual orientations between magnetization of the ferromagnetic film and the spin of a quasiparticle. Taking into account these phase shifts and repeating arguments similar to given above, one can show that the coefficients A and B in Eq.(2.4) acquire additional factors $\cos \left(2 \varphi_{H}\right)$ and $\cos \left(4 \varphi_{H}\right)$, respectively. At the point of " $0 "$ - " $\pi$ " transition the coefficient $A=0$, that is $\varphi_{H}=\pi / 4$. As a result, $\cos \left(4 \varphi_{H}\right)$ provides an additional factor, which changes the sign of the second harmonic amplitude $B$ in SFS structures from negative to positive.

In the present study we will show that contrary to SFS devices with standard geom- 
etry, it's possible to realize $\varphi$-junctions in the structures shown in Fig. 1.1. Qualitatively, these structures are superpositions of parallel SNS and SFS-channels, where supercurrent $I_{S}(\varphi)$ can be decomposed into two parts, $I_{N}(\varphi)$ and $I_{F}(\varphi)$, flowing across $\mathrm{N}$ and $\mathrm{F}$ films, respectively. For $L \ll \xi_{N}$ and at sufficiently low temperatures $I_{N}(\varphi)$ has large negative second CPR harmonic $B_{N}$. For $L>\xi_{1}$ supercurrent in the SFS-channel exhibits damped oscillations as a function of $L$. In this regime the second harmonic of CPR is negligibly small compared to the first one. Large difference between decay lengths of superconducting correlations in $\mathrm{N}$ and F-materials allows one to enter the regime when $\xi_{1}<L<\xi_{N}$. In this case the first CPR harmonic $A=A_{N}+A_{F}$ can be made small enough due to negative sign of $A_{F}$, while the second $\mathrm{CPR}$ harmonic $B \approx B_{N}$ is negative, thus making it possible to fulfill the condition (1.2). Note that we are considering here the regime of finite interface transparencies, when higher order harmonics decay fast with the harmonic order. Therefore, it is sufficient to consider only the first and the second harmonics of the CPR in all our subsequent discussions.

We show below that the mechanism described above indeed works in the considered S-FN-S junctions, and we estimate corresponding parameter range when $\varphi$-states can be realized.

\subsection{Model}

We consider two types of symmetric multilayered structures shown schematically on Fig.1.1. The structures consist of a superconducting (S) electrode contacting either the endwall of a FN bilayer (ramp type junctions) or the surface of $\mathrm{F}$ or $\mathrm{N}$ films (overlap junction geometry). The FN bilayer consists of ferromagnetic (F) film and normal metal (N) having a thickness $d_{F}$, and $d_{N}$ respectively. We suppose that the conditions of a dirty limit are fulfilled for all metals and that effective electron-phonon coupling constant is zero in $\mathrm{F}$ and $\mathrm{N}$ films. For simplicity we assume that the parameters $\gamma_{B N}$ and $\gamma_{B F}$ which characterize the transparencies of NS and FS interfaces are large enough

$$
\begin{aligned}
& \gamma_{B N}=\frac{R_{B N} \mathcal{A}_{B N}}{\rho_{N} \xi_{N}} \gg \frac{\rho_{S} \xi_{S}}{\rho_{N} \xi_{N}}, \\
& \gamma_{B F}=\frac{R_{B F} \mathcal{A}_{B F}}{\rho_{F} \xi_{F}} \gg \frac{\rho_{S} \xi_{S}}{\rho_{F} \xi_{F}},
\end{aligned}
$$

in order to neglect suppression of superconductivity in $\mathrm{S}$ parts of the junctions. Here $R_{B N}, R_{B F}$ and $\mathcal{A}_{B N}, \mathcal{A}_{B F}$ are the resistances and areas of the SN and SF interfaces, $\xi_{S}, \xi_{N}$ 
and $\xi_{F}$ are the decay lengths of S, N, F materials and $\rho_{S}, \rho_{N}$ and $\rho_{F}$ are their resistivities.

Under the above conditions the problem of calculation of the supercurrent in the structures reduces to solution of the set of Usadel equations [19,21,22]

$$
\frac{\xi^{2}}{G_{\omega}} \partial\left[G_{\omega}^{2} \partial \Phi_{\omega}\right]-\frac{\widetilde{\omega}}{\pi T_{C}} \Phi_{\omega}=0, G_{\omega}=\frac{\widetilde{\omega}}{\sqrt{\widetilde{\omega}^{2}+\Phi_{\omega} \Phi_{-\omega}^{*}}},
$$

where $\Phi_{\omega}$ and $G_{\omega}$ are Usadel Green's functions in $\Phi$ parametrization. They are $\Phi_{\omega, N}$ and $G_{\omega, N}$ or $\Phi_{\omega, F}$ and $G_{\omega, F}$ in N and F films correspondingly, $\omega=\pi T(2 m+1)$ are Matsubara frequencies $(\mathrm{m}=0,1,2, \ldots), \widetilde{\omega}=\omega+i H, H$, is exchange field of ferromagnetic material, $\xi^{2}=\xi_{N, F}^{2}=D_{N, F} / 2 \pi T_{C}$ for $\mathrm{N}$ and $\mathrm{F}$ layers respectively, $D_{N, F}$ are diffusion coefficients, $\partial=(\partial / \partial x, \partial / \partial z)$ is $2 \mathrm{D}$ gradient operator. To write equations $(1.4)$, we have chosen the $z$ and $x$ axis in the directions, respectively, perpendicular and parallel to the plane of $\mathrm{N}$ film and we have set the origin in the middle of structure at the free interface of F-film (see Fig.1.1).

The supercurrent $I_{S}(\varphi)$ can be calculated by integrating the standard expressions for the current density $j_{N, F}(\varphi, z)$ over the junction cross-section:

$$
\begin{aligned}
& \frac{2 e j_{N, F}(\varphi, z)}{\pi T}=\sum_{\omega=-\infty}^{\infty} \frac{i G_{\omega}^{2}}{\rho_{N, F} \widetilde{\omega}_{N, F}^{2}}\left[\Phi_{\omega} \frac{\partial \Phi_{-\omega}^{*}}{\partial x}-\Phi_{-\omega}^{*} \frac{\partial \Phi_{\omega}}{\partial x}\right] \\
& I_{S}(\varphi)=W \int_{0}^{d_{F}} j_{F}(\varphi, z) d z+W \int_{d_{F}}^{d_{F}+d_{N}} j_{N}(\varphi, z) W d z
\end{aligned}
$$

where $W$ is the width of the junctions, which is supposed to be small compared to Josephson penetration depth. It is convenient to perform the integration in (1.5) in F and $\mathrm{N}$ layers separately along the line located at $x=0$, where $z$-component of supercurrent density vanishes by symmetry.

Eq.(1.4) must be supplemented by the boundary conditions [23]. Since these conditions link the Usadel Green's functions corresponding to the same Matsubara frequency $\omega$, we may simplify the notations by omitting the subscript $\omega$. At the NF interface the boundary conditions have the form:

$$
\begin{gathered}
\gamma_{B F N} \xi_{F} \frac{\partial \Phi_{F}}{\partial z}=-\frac{G_{N}}{G_{F}}\left(\Phi_{F}-\frac{\widetilde{\omega}}{\omega} \Phi_{N}\right), \\
\gamma_{B N F} \xi_{N} \frac{\partial \Phi_{N}}{\partial z}=\frac{G_{F}}{G_{N}}\left(\Phi_{N}-\frac{\omega}{\widetilde{\omega}} \Phi_{F}\right), \\
\gamma_{B F N}=\frac{R_{B F N} \mathcal{A}_{B F N}}{\rho_{F} \xi_{F}}=\gamma_{B N F} \frac{\rho_{F} \xi_{F}}{\rho_{N} \xi_{N}}
\end{gathered}
$$

where $R_{B F N}$ and $\mathcal{A}_{B F N}$ are the resistance and area of the NF interface. 
The conditions at free interfaces are

$$
\frac{\partial \Phi_{N}}{\partial n}=0, \frac{\partial \Phi_{F}}{\partial n}=0
$$

The partial derivatives in (1.7) are taken in the direction normal to the boundary, so that $n$ can be either $z$ or $x$ depending on the particular geometry of the structure.

In writing the boundary conditions at the interface with a superconductor, we must take into account the fact that in our model we have ignored the suppression of superconductivity in electrodes, so that in superconductor

$$
\Phi_{S}( \pm L / 2)=\Delta \exp ( \pm i \varphi / 2), G_{S}=\frac{\omega}{\sqrt{\omega^{2}+\Delta^{2}}}
$$

where $\Delta$ is magnitude of the order parameter in S banks. Therefore for NS and FS interfaces we may write:

$$
\begin{aligned}
\gamma_{B N} \xi_{N} \frac{\partial \Phi_{N}}{\partial n} & =\frac{G_{S}}{G_{N}}\left(\Phi_{N}-\Phi_{S}( \pm L / 2)\right), \\
\gamma_{B F} \xi_{F} \frac{\partial \Phi_{F}}{\partial n} & =\frac{G_{S}}{G_{F}}\left(\Phi_{F}-\frac{\widetilde{\omega}}{\omega} \Phi_{S}( \pm L / 2)\right) .
\end{aligned}
$$

As in Eq. (1.7), $n$ in Eqs. (1.9a), (1.9b) is a normal vector directed into material marked at derivative.

For the structure presented in Fig.1.1a, the boundary-value problem (1.4) - (1.9b) was solved analytically in the linear approximation [47,48], i.e. under conditions

$$
G_{N} \equiv \operatorname{sgn}(\omega), G_{F} \equiv \operatorname{sgn}(\omega)
$$

In the present study we will go beyond linear approximation where qualitatively new effects are found.

\subsection{Ramp-type geometry}

The ramp type Josephson junction has simplest geometry among the structures shown in Fig.1.1. It consists of the NF bilayer, laterally connected with superconducting electrodes (see Fig.1.1a).

Properties of the considered structure are significantly different in the two opposite limits of thin or thick N- and F- films, respectively. In the case of thin films properties of the 


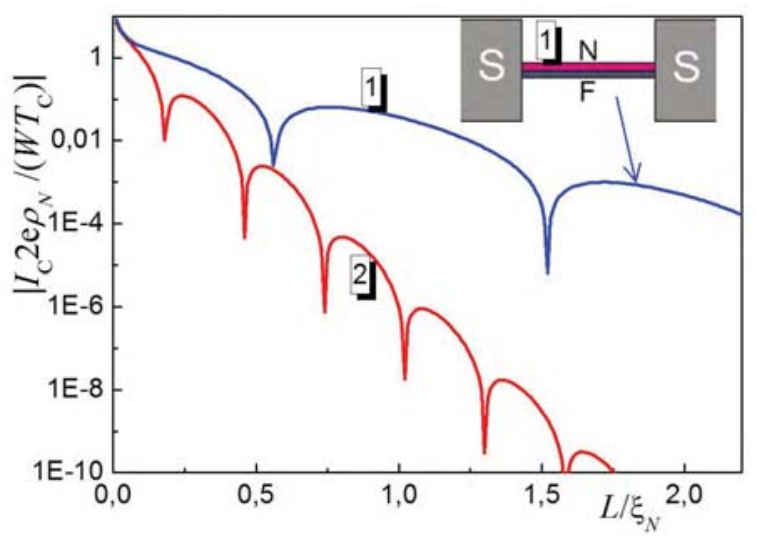

a)

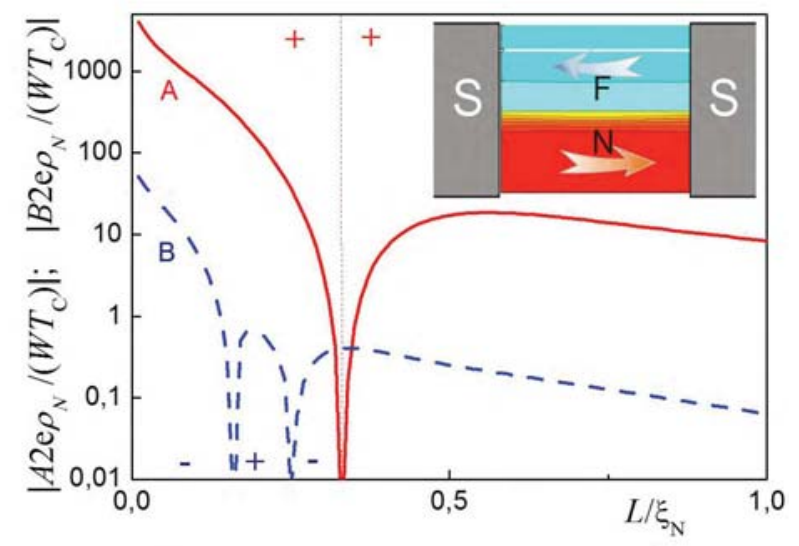

b)

Figure 1.3. (a) Normalized critical current $I_{C}$ versus normalized electrodes spacing $L$ for SFS structure with single F film (2) and for heterostructures with thin ferromagnetic/normal metal bilayer (1). (b) Harmonic amplitudes A(solid) and B(dashed line) in CPR for S-NF-S structure versus normalized electrodes spacing L for heterostructures with thick ferromagnetic/normal metal bilayer. Inset presents current distribuion calculated for $L=0.33 \xi_{N}$, for the case of $\varphi$-junction existence. The colors in the inset mean the intensity and sign of current density concentration in the horizontal direction.

structure resemble the properties of the SFS junction with effectively increased coherence length(see Fig.1.3a). For relatively thick films the weak link region may separate on the areas conducting the supercurrent in the opposite directions. The current density map along S-NF-S two-dimensional structure is shown in the inset in e Fig.1.3b). The calculations give that in considered junctions the first harmonic amplitudes in the CPR, A, may be equal to zero either due to cancellation of the contributions of current in F- and N- channels, if they are in $\pi$-state and in 0-state, respectively Hence, for selected bilayer parameters (mainly for selected thickness $\mathrm{dN}, \mathrm{dF}$ of $\mathrm{N}$ - and $\mathrm{F}$ - layers) one can obtain real $\varphi$-junction with strongly nonsinusoidal CPR with negative amplitude of the second harmonic.

In general case, there are three characteristic decay lengths in the considered structure [47], [47], [68]. They are $\xi_{N}, \xi_{H}=\xi_{1}+i \xi_{2}$, and $\zeta=\zeta_{1}+i \zeta_{2}$. The first two lengths determine the decay and oscillations of superconducting correlations far from FN interface, while the last one describes their behavior in its vicinity. Similar length scale $\zeta$ occurs in a vicinity of a domain wall [60] $]^{-}[68]$. In the latter, exchange field is averaged out for antiparallel directions of magnetizations, and the decay length of superconducting correlations becomes close to $\xi_{N}$. At FN interface, the flow of spin-polarized electrons from $\mathrm{F}$ to $\mathrm{N}$ metal and reverse flow of unpolarized electrons from $\mathrm{N}$ to $\mathrm{F}$ suppresses the exchange field in its vicinity to a value smaller than that in a bulk ferromagnetic material thus providing the existence of $\zeta$. Under certain set of parameters [47] these lengths, $\zeta_{1}$, and, $\zeta_{2}$, can 
become comparable to $\xi_{N}$, which is typically much larger than $\xi_{1}$ and $\xi_{2}$, which are equal to $\xi_{F} \sqrt{\pi T_{C} / H}$ for $H \gg \pi T_{C}$.

The existence of three decay lengths, $\xi_{N}, \zeta$, and $\xi_{H}$, should lead to appearance of three contributions to total supercurrent, $I_{N}, I_{F N}$ and $I_{F}$, respectively. The main contribution to $I_{N}$ component comes from a part of the supercurrent uniformly distributed in a normal film. In accordance with the qualitative analysis carried out in Section II, it is the only current component which provides a negative value of the amplitude of the second harmonic $B$ in the current-phase relation. The smaller the distance between electrodes $L$, the larger this contribution. To realize a $\varphi$-contact, one must compensate for the amplitude of the first harmonic, $A$, in a total current to a value that satisfies the requirement (1.2). Contribution to $A$ from $I_{N}$ also increases with decreasing $L$. Obviously, it's difficult to suppress the coefficient $A$ due to the $I_{F N}$ contribution only, since $I_{F N}$ flows through thin near-boundary layer. Therefore, strong reduction of $A$ required to satisfy the inequality (1.2) can only be achieved as a result of compensation of the currents $I_{N}$ and $I_{F}$ flowing in opposite directions in $\mathrm{N}$ and $\mathrm{F}$ films far from FN interface. Note that the oscillatory nature of the $I_{F}(L)$ dependence allows to satisfy requirement $(1.2)$ in a certain range of $L$. The role of $I_{F N}$ in a balance between $I_{N}$ and $I_{F}$ can be understood by solving the boundary value problem $(1.4)$ - (1.9b) which admits an analytic solution in some limiting cases.

\subsubsection{Limit of small $L$}

Solution of the boundary-value problem (1.4)-(1.9b) can be simplified in the limit of small distance between superconducting electrodes

$$
L \ll \min \left\{\xi_{1}, \xi_{N}\right\}
$$

In this case one can neglect non-gradient terms in (1.4) and obtain that contributions to the total current resulting from the redistribution of currents near the FN interface cancel each other leading to $I_{F N}=0$ (see Appendix 1.8.1 for the details). As a result, the total current $I_{S}(\varphi)$ is a sum of two terms only

$$
\begin{gathered}
I_{S}(\varphi)=I_{N}(\varphi)+I_{F}(\varphi) \\
\frac{2 e I_{N}(\varphi)}{\pi T W d_{N}}=\frac{1}{\gamma_{B N} \xi_{N} \rho_{N}} \sum_{\omega=-\infty}^{\infty} \frac{\Delta^{2} G_{N} G_{S} \sin (\varphi)}{\omega^{2}}
\end{gathered}
$$




$$
\frac{2 e I_{F}(\varphi)}{\pi T W d_{F}}=\frac{1}{\gamma_{B F} \xi_{F} \rho_{F}} \sum_{\omega=-\infty}^{\infty} \frac{\Delta^{2} G_{N} G_{S} \sin (\varphi)}{\omega^{2}}
$$

where $G_{N}=\frac{\omega}{\sqrt{\omega^{2}+\Delta^{2} \cos ^{2}\left(\frac{\varphi}{2}\right)}}$. The currents $I_{N}(\varphi)$ and $I_{F}(\varphi)$ flow independently across $\mathrm{F}$ and $\mathrm{N}$ parts of the weak link. The $I_{N, F}(\varphi)$ dependencies coincide with those calculated previously for double-barrier junctions [23] in the case when $L$ lies within the interval defined by the inequalities (1.11).

It follows from $(1.12),(1.13)$ that in the considered limit neither the presence of a sharp FN boundary in the weak link region, nor strong difference in transparencies of SN and SF interfaces lead to intermixing of the supercurrents flowing in the $\mathrm{F}$ and $\mathrm{N}$ channels. It is also seen that amplitude of the first harmonic of $I_{F}(\varphi)$ current component is always positive and the requirement (1.2) can not be achieved.

\subsubsection{Limit of intermediate $L$}

For intermediate values of spacing between the S electrodes

$$
\xi_{1} \ll L \ll \xi_{N}
$$

and for the values of suppression parameters at SN and SF interfaces satisfying the conditions (1.3), the boundary problem (1.4)-(1.9b) can be solved analytically for sufficiently large magnitude of suppression parameter $\gamma_{B F N}$. It is shown in Appendix 1.8.2 that under these restrictions in the first approximation we can neglect the suppression of superconductivity in the $\mathrm{N}$ film due to proximity with the $\mathrm{F}$ layer and find that

$$
\Phi_{N}=\Delta \cos \left(\frac{\varphi}{2}\right)+i \frac{\Delta G_{S} \sin \left(\frac{\varphi}{2}\right)}{\gamma_{B N} G_{N}} \frac{x}{\xi_{N}}, G_{N}=\frac{\omega}{\sqrt{\omega^{2}+\Delta^{2} \cos ^{2}\left(\frac{\varphi}{2}\right)}}
$$

while spatial distribution of $\Phi_{F}(x, z)$ includes three terms: the first two describe the influence of the $\mathrm{N}$ film, while the last one has the form well known for SFS junctions [20], [21]' [22].

Substitution of these solutions into expression for the supercurrent (1.5) leads to $I_{S}(\varphi)$ dependence consisting of three terms

$$
I_{S}(\varphi)=I_{N}(\varphi)+I_{F}(\varphi)+I_{F N}(\varphi) .
$$

Here $I_{N}(\varphi)$ is the supercurrent across the N layer. In the considered approximation $I_{N}(\varphi)$ is given by the expression (1.12). The second term in (1.16) equals to supercurrent across 
SFS double barrier structure in the limit of small transparencies of SF interfaces [59]' [70]

$$
\frac{2 e I_{F}(\varphi)}{\pi T W d_{F}}=\frac{\Delta^{2} \sin (\varphi)}{\gamma_{B F}^{2} \xi_{F} \rho_{F}} \sum_{\omega=-\infty}^{\infty} \frac{G_{S}^{2}}{\omega^{2} \sqrt{\widetilde{\Omega}} \sinh \left(2 q_{L}\right)}
$$

where $q_{L}=L \sqrt{\widetilde{\Omega}} / 2 \xi_{F}, \widetilde{\Omega}=|\Omega|+i H \operatorname{sgn}(\Omega) / \pi T_{C}, \Omega=\omega / \pi T_{C}$.

The last contribution is shown in 1.8.2 to contain three components

$$
I_{F N}(\varphi)=I_{F N 1}(\varphi)+I_{F N 2}(\varphi)+I_{F N 3}(\varphi)
$$

with additional smallness parameters $\gamma_{B F N}^{-1}$ and $\gamma_{B F N}^{-1} \xi_{F} / \xi_{N}$ compared to the current $I_{F}(\varphi)$ given by Eq.(1.17). Nevertheless, these currents should be taken into account in the analysis because they decay significantly slower than $I_{F}(\varphi)$ with increasing $L$.

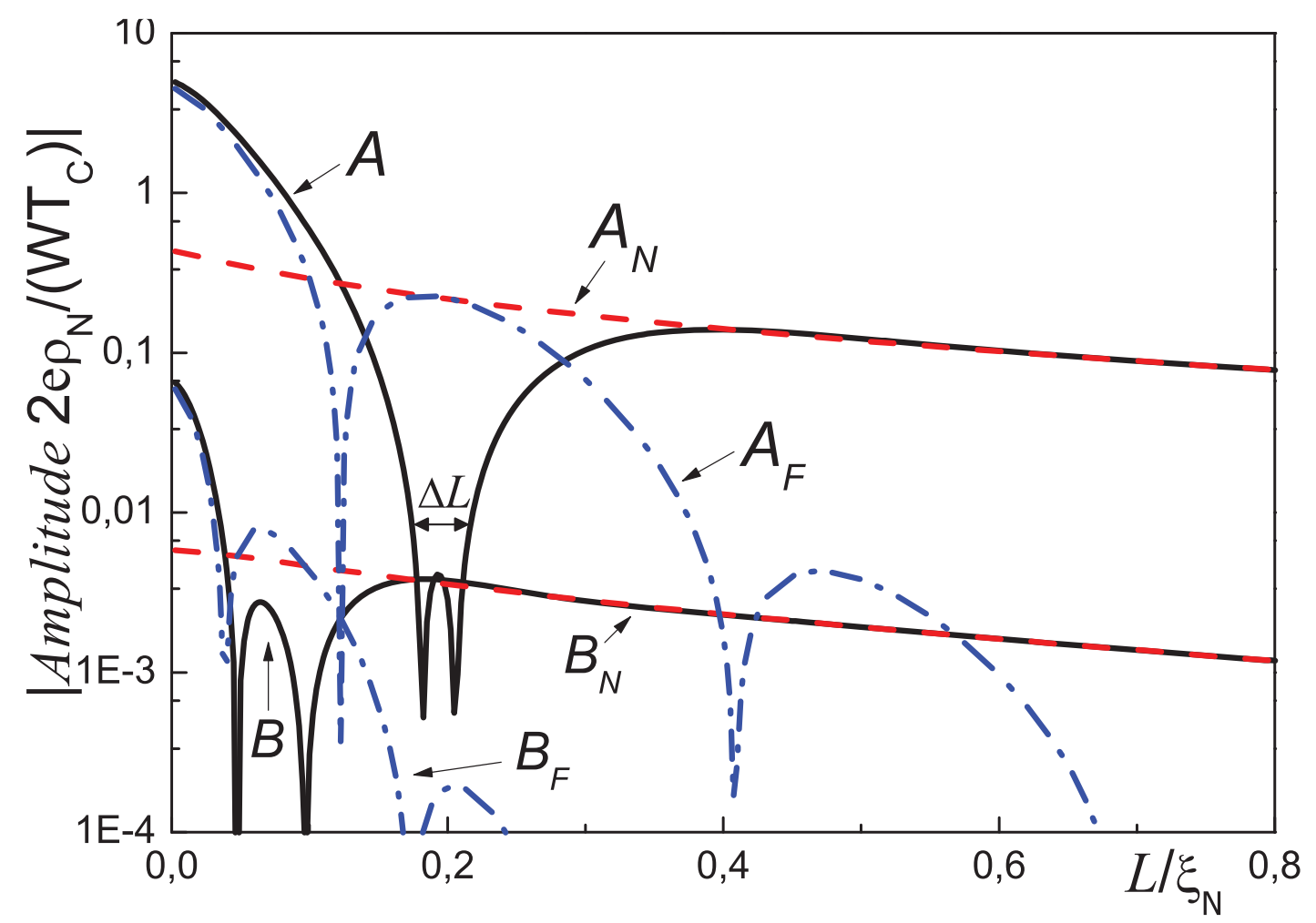

Figure 1.4. Numerically calculated amplitudes $A$ and $B$ in the CPR of ramp S-NF-S structure $\left(d_{N}=0.1 \xi_{N}, d_{F}=1.06 \xi_{N}\right)$ and their components $A_{N}, A_{F}, B_{N}, B_{F}$ versus electrode spacing $L$ at $T=0.7 T_{C}$. In correspondence with Fig.1.6 parameters are chosen to form enhanced $\varphi$-state interval marked by " $\Delta L "$.

\subsection{3 $\varphi$-state existence}

The conditions for the implementation of a $\varphi$-contact are the better, the larger the relative amplitude of the second harmonic which increases at low temperatures. Therefore, 


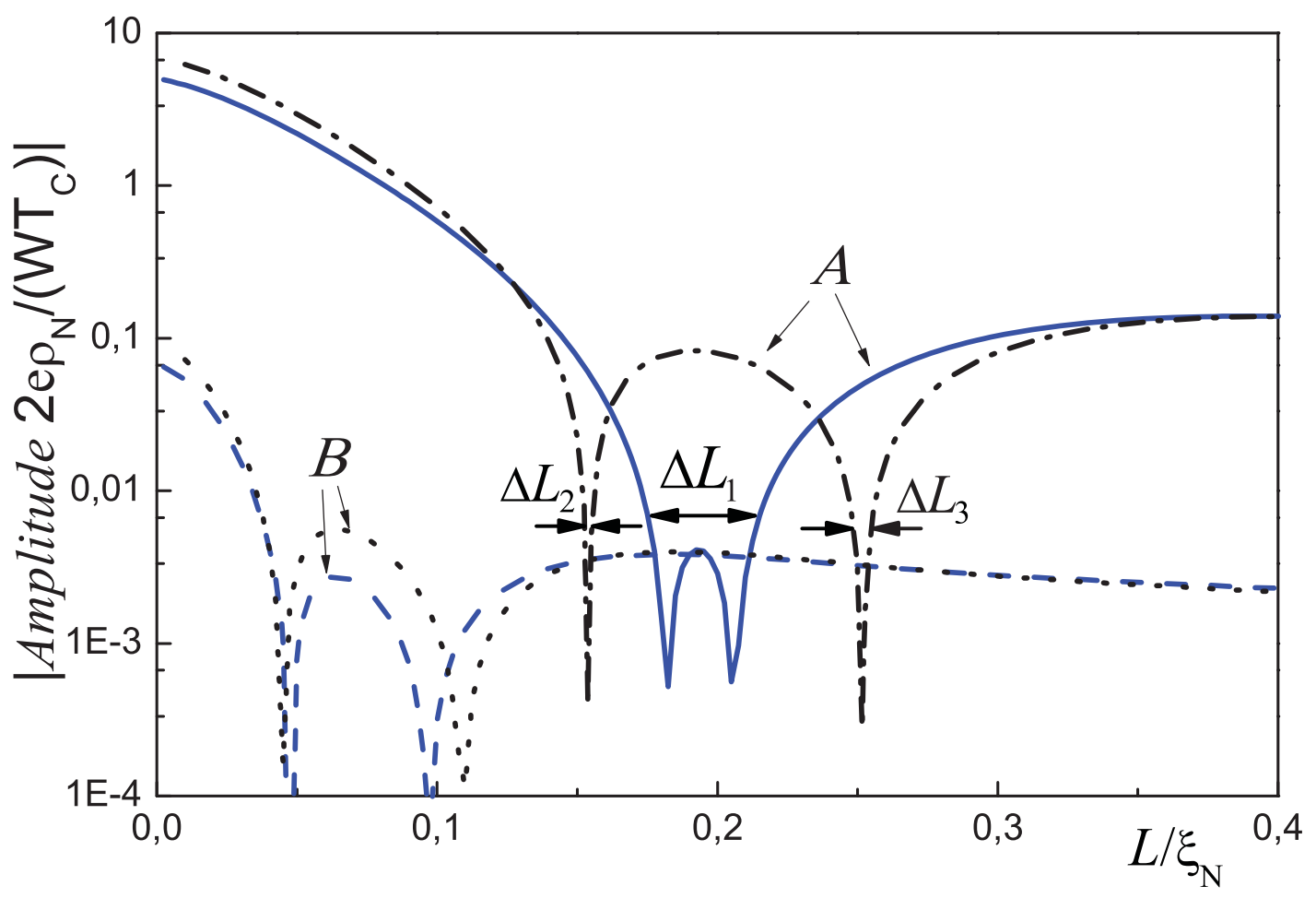

Figure 1.5. Numerically calculated CPR amplitudes $A$ and $B$ versus electrode spacing $L$ for S-FN-S structures with $d_{F}=1.06 \xi_{N}$ (solid and dashed lines respectively) and $d_{F}=1.4 \xi_{N}$ (dash-dotted and dotted lines). It is clear that enhanced $\varphi$-interval $\Delta L_{1}$ formed in the first case is much larger than pair of ordinary $\varphi$-intervals $\Delta L_{2}$ and $\Delta L_{3}$ in the second one.

low temperature regime is most favorable for a $\varphi$-state. In the limit $T \ll T_{C}$ we can go from summation to integration over $\omega$ in (1.12), (1.17), (1.74)- (1.76). From (1.12) we have

$$
\frac{2 e I_{N}(\varphi)}{W d_{N}}=\frac{\Delta}{\gamma_{B N} \xi_{N} \rho_{N}} K\left(\sin \frac{\varphi}{2}\right) \sin (\varphi)
$$

where $K(x)$ is the complete elliptic integral of the first kind. Expanding expression (1.19) in the Fourier series it is easy to obtain

$$
\begin{gathered}
A_{N}=Q_{0} \frac{8}{\pi} \int_{0}^{1} x^{2} \sqrt{1-x^{2}} K(x) d x=\Upsilon_{A} Q_{0}, \\
B_{N}=2 A_{N}-\frac{32}{\pi} Q_{0} \int_{0}^{1} x^{4} \sqrt{1-x^{2}} K(x) d x=\Upsilon_{B} Q_{0},
\end{gathered}
$$


where $Q_{0}=\Delta W d_{N} / e \gamma_{B N} \xi_{N} \rho_{N}, A_{N}, B_{N}$ are the first and the second harmonic amplitudes of $I_{N}(\varphi)$,

$$
\begin{aligned}
& \Upsilon_{A}=\frac{2 \pi^{2}}{\Gamma^{2}\left(-\frac{1}{4}\right) \Gamma^{2}\left(\frac{7}{4}\right)} \simeq 0.973 \\
& \Upsilon_{B}=2 \Upsilon_{A}-\frac{\pi}{2}{ }_{3} F_{2}\left(\frac{1}{2}, \frac{1}{2}, \frac{5}{2} ; 1,4 ; 1\right) \simeq-0.146
\end{aligned}
$$

where $\Gamma(z)$ is Gamma-function and ${ }_{p} F_{q}$ is generalized hypergeometric function.

Evaluation of the sums in (1.17), (1.74)- (1.76) can be done for $H \gg \pi T_{C}$ and $T \ll T_{C}$ resulting in $I_{F}(\varphi)=A_{F} \sin (\varphi)$ with

$$
A_{F}=P_{0} \frac{2}{\sqrt{h}} \exp (-\kappa L) \cos \left(\kappa L+\frac{\pi}{4}\right)
$$

$\kappa=\sqrt{h} / \sqrt{2} \xi_{F}, h=H / \pi T_{C}$ and $P_{0}=\Delta W d_{F} / e \gamma_{B F}^{2} \xi_{F} \rho_{F}$. Substitution of (1.20), (1.21) into the inequalities (1.2) gives $\varphi$-state requirements for ramp-type structure

$$
\begin{gathered}
\left|\Upsilon_{A}+\frac{1}{\varepsilon} \Psi(L)\right|<2\left|\Upsilon_{B}\right|, \varepsilon=\frac{\sqrt{h} \gamma_{B F}^{2}}{2 \gamma_{B N}} \frac{d_{N} \xi_{F} \rho_{F}}{d_{F} \xi_{N} \rho_{N}}, \\
\Psi(L)=\exp (-\kappa L) \cos \left(\kappa L+\frac{\pi}{4}\right) .
\end{gathered}
$$

This expression gives the limitation on geometrical and materials parameters of the considered structures providing the existence of $\varphi$-junction. Function $\Psi(L)$ has the first minimum at $\kappa L=\pi / 2, \Psi(\pi / 2 \kappa) \approx-0.147$. For large values of $\varepsilon$ inequality (1.23) can not be fulfilled at any length $L$. Thus solutions exist only in the area with upper limit

$$
\varepsilon<\frac{-\Psi(\pi / 2 \kappa)}{\Upsilon_{A}-2\left|\Upsilon_{B}\right|} \approx 0.216
$$

At $\varepsilon \approx 0.216$ the left hand side of inequality (1.23) equals to its right hand part providing the nucleation of an interval of $\kappa L$ in which we can expect the formation of a $\varphi$-contact. This interval increases with decrease of $\varepsilon$ and achieves its maximum length

$$
1.00 \lesssim \kappa L \lesssim 2.52
$$

at $\varepsilon=\frac{-\Psi(\pi / 2 \kappa)}{\Upsilon_{A}+2\left|\Upsilon_{B}\right|} \approx 0.116$. It is necessary to note that at $\varepsilon=-\Psi(\pi / 2 \kappa) / \Upsilon_{A} \approx 0.151$ there is a transformation of the left hand side local minimum in (1.23), which occurs at $\kappa L=\pi / 2$, into local maximum; so that at $\varepsilon \approx 0.116$ the both sides of (1.23) become equal to each other, and the interval (1.25) of $\varphi$-junction existence subdivides into two parts. With a 
further decrease of $\varepsilon$ these parts are transformed into narrow bands, which are localized in the vicinity of the $0-\pi$ transition point $\left(A_{N}+A_{F}=0\right)$; they take place at $\kappa L=\pi / 4$ and $\kappa L=5 \pi / 4$. The width of the bands decreases with decrease of $\varepsilon$.

Thus, our analysis has shown that for

$$
0.12 \lesssim \varepsilon \lesssim 0.2
$$

we can expect the formation of $\varphi$-junction in a sufficiently wide range of distances $\Delta L$ between the electrodes determined by (1.23). Now we will take into the account the impact of the interface term $I_{F N}(\varphi)$. In the considered approximations, it follows from (1.74)(1.76) that

$$
\begin{gathered}
I_{F N 1}(\varphi)=\frac{2 U_{0} \xi_{F} \exp \left(-\frac{\kappa L}{2}\right) \cos \left(\frac{\kappa L}{2}-\frac{\pi}{4}\right)}{\gamma_{B F} \gamma_{B N} \xi_{N} h^{3 / 2}} \sin (\varphi), \\
I_{F N 2}(\varphi)=-\frac{\sqrt{2} U_{0} \xi_{F}}{4 h^{3 / 2} \gamma_{B N} \gamma_{B F N} \xi_{N}} \sin (\varphi) K\left(\sin \frac{\varphi}{2}\right), \\
I_{F N 3}(\varphi)=-\frac{2 U_{0} \exp \left(-\frac{\kappa L}{2}\right) \sin \left(\frac{\kappa L}{2}\right)}{h \gamma_{B F}} \sin (\varphi) K\left(\sin \frac{\varphi}{2}\right),
\end{gathered}
$$

where $U_{0}=\Delta W / e \gamma_{B F N} \rho_{F}$. In the range of distances between the electrodes $\pi / 4<\kappa L<$ $5 \pi / 4$ currents $I_{F N 2}(\varphi)$ and $I_{F N 3}(\varphi)$ are negative. These contributions have the same form of CPR as it is for the $I_{N}(\varphi)$ term, and due to negative sign suppress the magnitude of supercurrent across the junction thus making the inequality (1.23) easier to perform. The requirement $B<0$ imposes additional restriction on the value of the suppression parameter $\gamma_{B F N}$

$$
\gamma_{B F N}>\frac{\rho_{N} \xi_{N}}{h d_{N} \rho_{F}}\left(\frac{\xi_{F}}{\xi_{N} \gamma_{B F N} h^{1 / 2}}+\frac{\gamma_{B N}}{\gamma_{B F}}\right)
$$

In derivation of this inequality we have used the fact that in the range of distances between the electrodes $\pi / 4<\kappa L<5 \pi / 4$ depending on $\kappa L$ factor in (1.29) is of the order of unity. It follows from (1.30) that for a fixed value of $\gamma_{B F N}$ domain of $\varphi$-junction existence extends with increase of thickness of normal films $d_{N}$ and this domain disappears if $d_{N}$ becomes smaller than the critical value, $d_{N C}$,

$$
d_{N C}=\frac{\rho_{N} \xi_{N}}{h \rho_{F} \gamma_{B F N}}\left(\frac{\xi_{F}}{\xi_{N} \gamma_{B F N} h^{1 / 2}}+\frac{\gamma_{B N}}{\gamma_{B F}}\right) .
$$

The existence of the critical thickness $d_{N C}$ follows from the fact that the amplitude $B$ in 


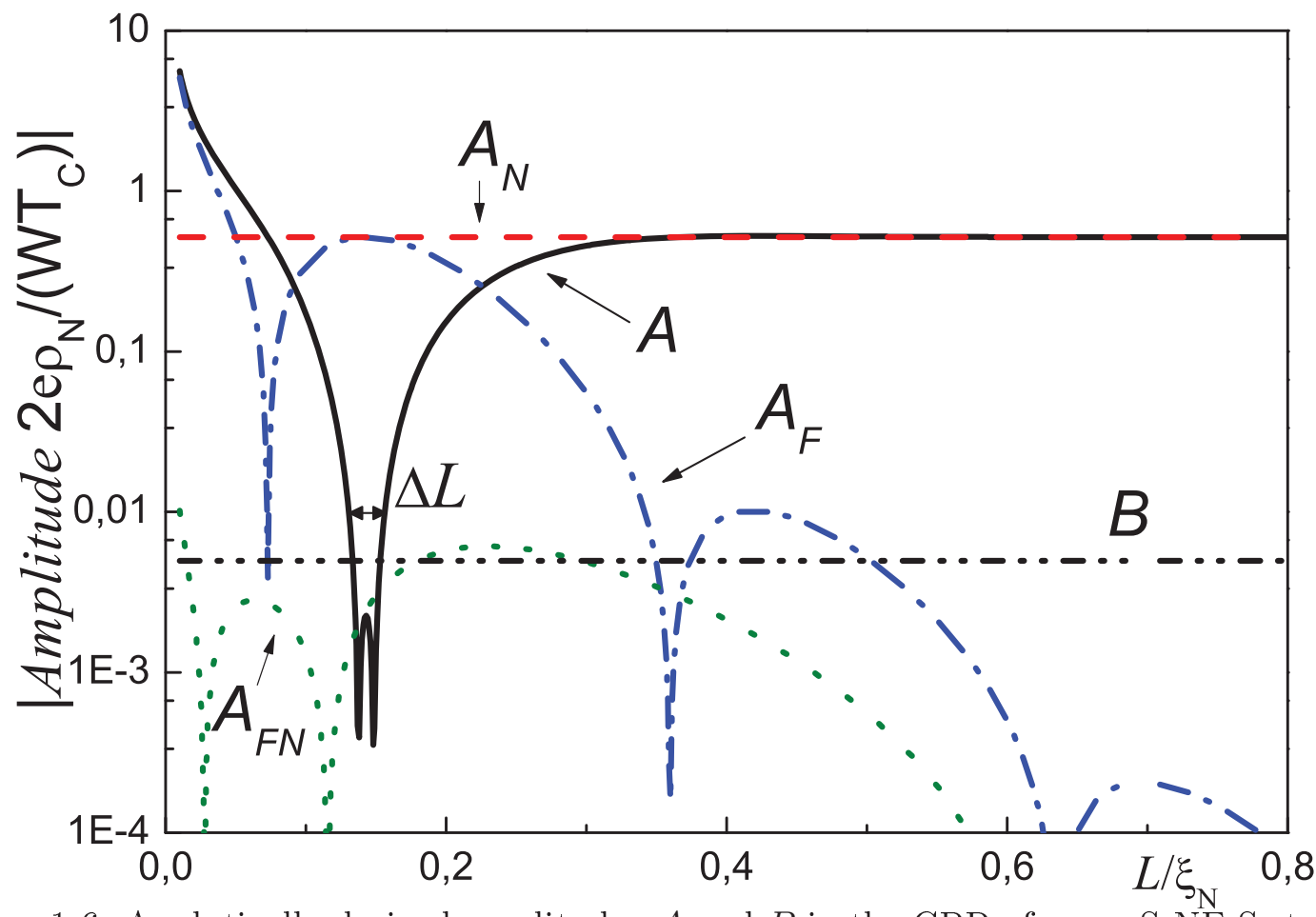

Figure 1.6. Analytically derived amplitudes $A$ and $B$ in the CPR of ramp S-NF-S structure $\left(d_{N}=0.1 \xi_{N}, d_{F}=0.65 \xi_{N}\right)$ and their components $A_{N}, A_{F}, A_{F N}$ versus electrode spacing $L$ at $T=0.7 T_{C}$. Also enhanced interval of $\varphi$-state, $\Delta L$, is marked.

$I_{N}$ is proportional to $d_{N}$, while in $I_{F N}$ term the parameter $B$ is independent on $d_{N}$. The sign of $I_{F N 1}(\varphi)$ is positive for $\pi / 4<\kappa L<3 \pi / 4$ and negative for $3 \pi / 4<\kappa L<5 \pi / 4$ thus providing an advantage for a $\varphi$-junction realization for the lengths which belong to the second interval.

Figure 1.6 illustrates our analysis. The solid line in Fig.1.6 is the modulus of the amplitude of the first harmonic in CPR as a function of distance $L$ between $\mathrm{S}$ electrodes. It is the result of summation of the two contributions following from Eqs. (1.17) (dashdotted line) and (1.12) (dashed line). The dash-dot-dotted line in Fig 1.6 is the amplitude of the second harmonic of the CPR following from (1.12). The dotted line is $I_{F N}(L)$ calculated from (1.18), (1.74)- (1.76). All calculations have been done for a set of parameters $d_{N}=0.1 \xi_{N}, d_{F}=0.65 \xi_{N}, \gamma_{B N}=0.1, \gamma_{B F}=1, \gamma_{B N F}=10, \xi_{F}=0.1 \xi_{N}, \rho_{N}=\rho_{F}$, $T=0.7 T_{C}, H=10 T_{C}$. These parameters are close to those in real experimental situation. All the amplitudes were normalized on factor $\left(2 e \rho_{N} /\left(W T_{C}\right)\right)^{-1}$. It is evident that there is an interval of $L$, for which the currents in $\mathrm{N}$ and $\mathrm{F}$ layers flow in opposite directions. As a result of the addition of these currents the points of $0-\pi$ transitions start to be closer to each other. It is seen that in the entire region between these points, the inequality (1.2) is fulfilled. This is exactly the $L$-interval, inside which a $\varphi$-junction can be realized. It 


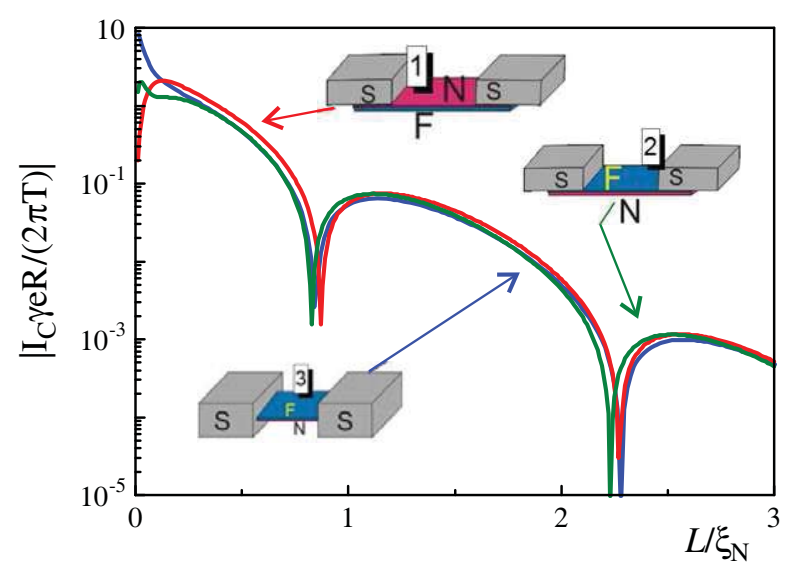

a)

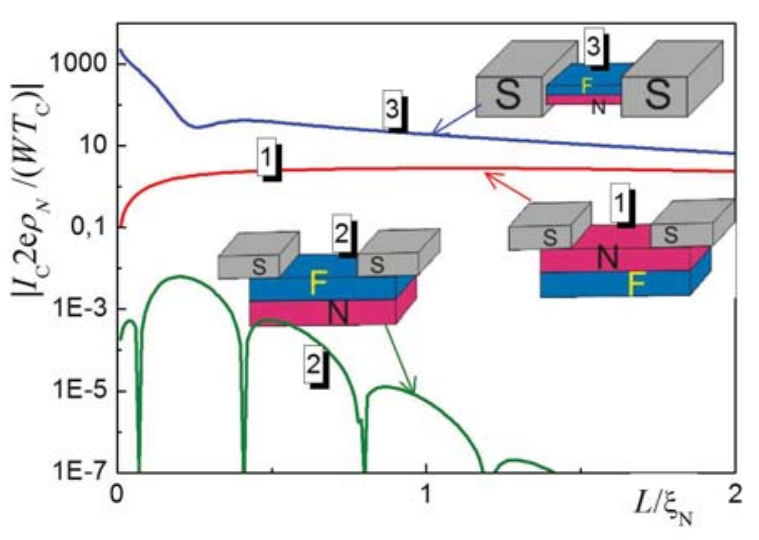

b)

Figure 1.7. Normalized critical current $I_{C}$ versus normalized electrodes spacing L for SNFNF-FNS (1), SFN-FN-NFS (2) and S-NF-S (3) structures in the limit of a) thin N- and Flayers $\left(d_{N}=d_{F}=0.01 \xi_{N}\right)$ and b) thick N- and F- layers $\left(d_{N}=d_{F}=3 \xi_{N}\right)$

is also seen that contribution of $I_{F N}$ part into the full current is small and in accordance with our analisys does not play a noticeable role.

The boundary problem (1.4)-(1.9b) has been solved numerically for the same set of junction parameters except $d_{F}$. The results of calculations for $d_{F}=1.06 \xi_{N}$ and $d_{F}=1.4 \xi_{N}$ are shown in Fig.1.4 and Fig.1.5. The solid lines in Fig.1.4 are the modulus of the amplitudes of the first, $A$, and the second, $B$, harmonic of CPR as a function of distance $L$ between $\mathrm{S}$ electrodes. The dashed and dash-dotted lines demonstrate the contributions to these amplitudes from the currents flowing in $\mathrm{N}$ and $\mathrm{F}$ films, respectively. All the amplitudes were normalized on the same factor $\left(2 e \rho_{N} /\left(W T_{C}\right)\right)^{-1}$. It is seen that the main difference between analytical solutions presented in Fig.1.6 and the curves calculated numerically are located in region of small $L$. It is also seen that amplitudes of first and second harmonics of the part of the current flowing in the $\mathrm{N}$ film slightly decay with $L$ increase. The points of $0-\pi$ transition of the first harmonic amplitude of the part of the current flowing in the F layer is slightly shifted to the right, toward larger $L$. It is also seen that the amplitude of the second harmonic, $B_{F}$, in the interval of interest in the vicinity of $L \approx 0.2 \xi_{N}$ is negligibly small compared to the magnitude of, $B_{N}$. As a result, the shape of $A(L)$ curves in Fig.1.6 and Fig.1.4 is nearly the same, with a little bit larger interval of $\varphi$-junction existence for the curve calculated numerically.

Figure 1.5 demonstrates the same $A(L)$ and $B(L)$ dependencies as in Fig.1.4 (solid and dashed lines) together with $A(L)$ and $B(L)$ curves calculated for $d_{F}=1.4 \xi_{N}$ (dashdoted and dotted lines). It is clearly seen that for larger $d_{F}$ we get out of the interval (1.26) 
and instead of relatively large zone $\Delta L_{1}$ may have $\varphi$-junction in two very narrow intervals $\Delta L_{2}$ and $\Delta L_{3}$ located in the vicinity of $0-\pi$ transitions of the first harmonic amplitude $A$.

\subsection{Overlap type geometry}

From technological point of view the overlap junction geometry presented in Fig.1.1b (the overlap length is much larger $\xi_{N}$ ) looks more reliable [11]. Our numerical calculations have shown that in the case of thin films and large transparency of FN interface it doesn't matter, whether the N- layer is above or behind the F-layer (see Fig 1.7a).

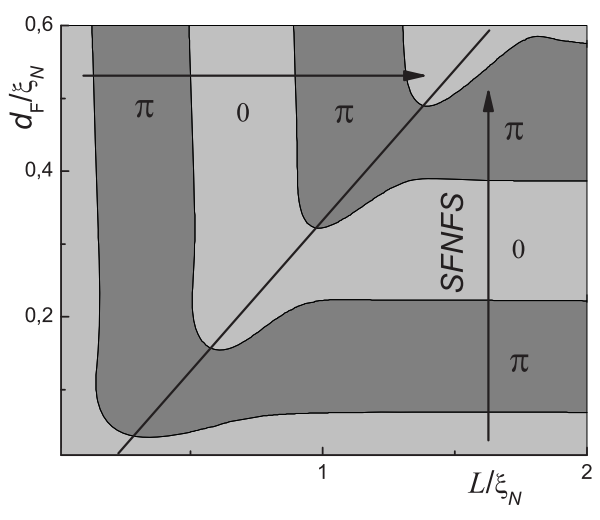

a)

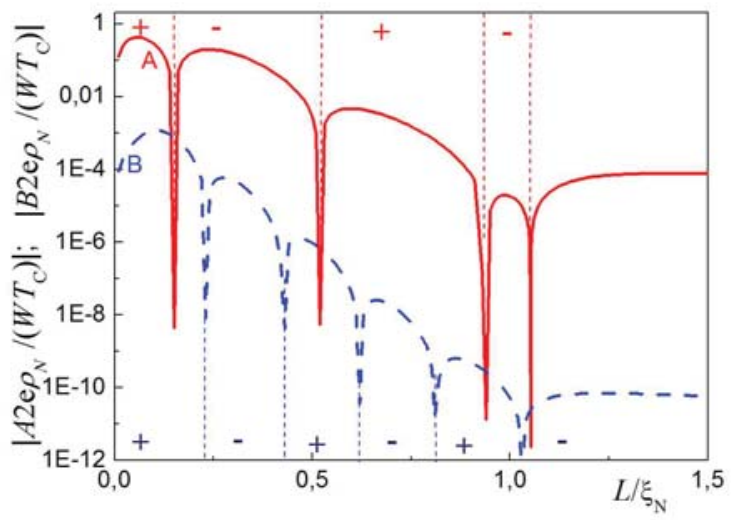

b)

Figure 1.8. a) Alternation of 0 and $\pi$-state areas on $\left(L, d_{F}\right)$ phase diagram in SFN-FN-NFS structure. b) Harmonic amplitudes A (solid) and B (dashed line) in CPR for SFN-FN-NFS structure versus junction thickness $L$ for heterostructures with thick ferromagnetic/normal metal bilayer.

At arbitrary film thickness the situation drastically changes. In SFN-FN-NFS junctions (Fig. 1.7a) the large difference between effective $\xi_{N}$ and $\xi_{F}$ results in formation of two (SFS and SFNFS) parallel current channels, which compete each other. In SNF-NF-FNS structures the current through long SNFNS channel is always much smaller than current through SNS part. In SFN-FN-NFS junctions the signs of the critical current $I_{C}$ and harmonic amplitudes in the SFS channel are controlled by the distance $L$ between $S$ electrodes. The sign of contribution to $I_{C}$ in SFNFS channel is a function of $\mathrm{F}$ layer thickness $d_{F}$. From presented in Fig.1.8 a phase diagram of the junctions harmonic amplitudes in $(L, d F)$ plane it is obviously follows that by proper choice of F-film geometry it is possible to suppress the amplitude of the first harmonic in CPR and to get the amplitude of the second one with the necessary sign for $\varphi$-junction existence (see Fig. 1.8b). 


\subsection{Ramp type overlap (RTO) junctions}

Conditions for the existence of $\varphi$-junction (1.25), (1.26) can be improved by slight modifications of contact geometry, namely, by using a combination of ramp and overlap configurations, as it is shown in Fig.1.1b. Fig.1.9a demonstrates numerically calculated spatial distribution of supercurrent in RTO $\varphi$-junction at Josephson phase $\varphi=\pi / 2$. The current density is presented by darkness and the arrows give flows directions. The relative smallness of the first harmonics amplitude is provided by opposite currents in $\mathrm{N}$ and $\mathrm{F}$ films. The main feature of the ramp-overlap geometry is seen to be specific current distribution in the normal layer leading to another CPR shape with dependence on thickness $d_{N}$. Further, the current $I_{N}$ should saturate as a function of $d_{N}$, since normal film regions located at distances larger than $\xi_{N}$ from SN interface are practically excluded from the process of supercurrent transfer due to exponential decay of proximity-induced superconducting correlations [71]. The specific geometry of the RTO structures makes theoretical analysis of the processes more complex than in ramp contact. Nevertheless, it is possible to find analytical expressions for supercurrent in these structures and to show that the range of parameters providing the existence of $\varphi$-state is broader than in the ramp type configuration.

To prove this statement, we consider the RTO structure in most practical case of thin $\mathrm{N}$ film

$$
d_{N} \ll \xi_{N}
$$

and sufficiently large $\gamma_{B F N}$ providing negligibly small suppression of superconductivity in $\mathrm{N}$ film due to proximity with $\mathrm{F}$ layer. We will assume additionally that electrode spacing $L$ is also small

$$
L \ll \xi_{N},
$$

in order to have nonsinusoidal CPR. Under these conditions we can at the first step consider the Josephson effect in overlap SN-N-NS structure. Then, at the second step we will use the obtained solutions to calculate supercurrent flowing across the F part of the RTO structure. The details of calculations are summarized in Appendices 1.8.3 and 1.8.4. They give that the supercurrent

$$
I_{S}(\varphi)=I_{N}(\varphi)+I_{F}(\varphi)+I_{F N}(\varphi)
$$

consists of three components. Expression for the part of current flowing across $\mathrm{N}$ film has 
the form

$$
\frac{2 e I_{N}(\varphi)}{\pi T W d_{N}}=\frac{2}{\rho_{N} \xi_{N} \sqrt{\gamma_{B M}}} \sum_{\omega=-\infty}^{\infty} \frac{r^{2} \delta^{2} \sin \varphi \sqrt{\left(\Omega \gamma_{B M}+G_{S}\right)}}{\sqrt{2 \Omega \mu^{2}\left(\sqrt{\Omega^{2}+r^{2} \delta^{2}}+\mu\right)}}
$$

where $r=G_{S} /\left(\Omega \gamma_{B M}+G_{S}\right), \gamma_{B M}=\gamma_{B N} d_{N} / \xi_{N}$ and $\mu=\sqrt{\Omega^{2}+r^{2} \delta^{2} \cos ^{2}(\varphi / 2)}, \delta=$ $\Delta / \pi T_{C}$

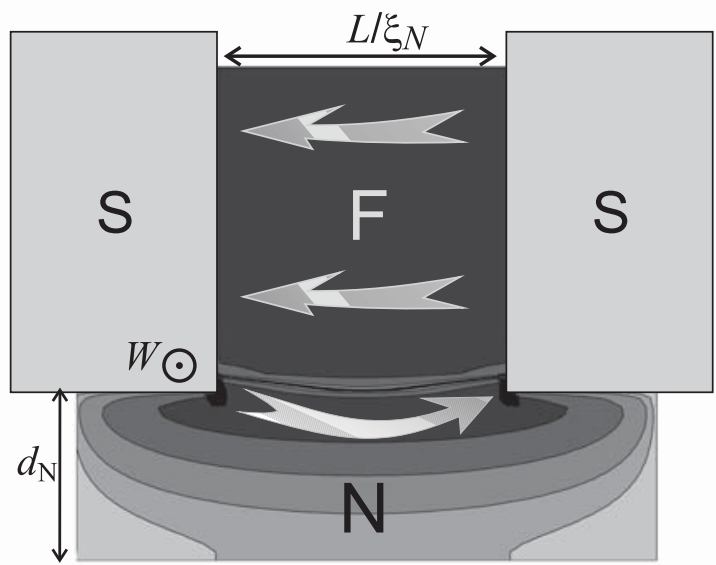

a)

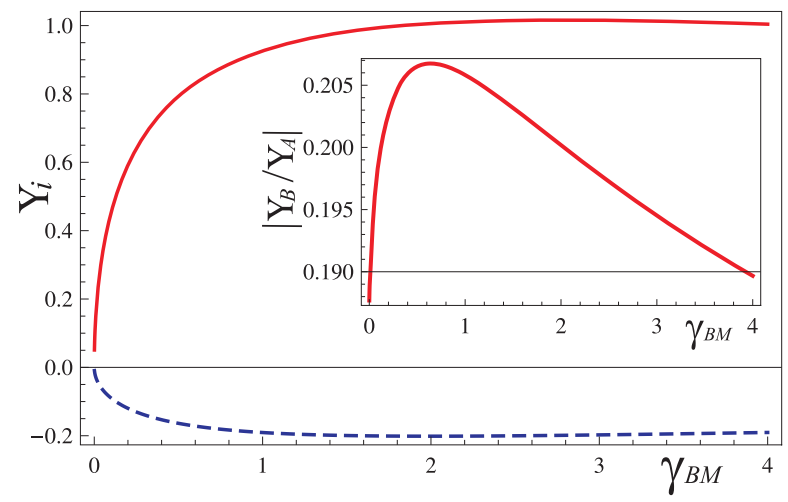

b)

Figure 1.9. a) Current distribution along RTO-type SN-FN-NS structure at $L=0.63 \xi_{N}$, $d_{N}=\xi_{N}, d_{F}=2 \xi_{N}$ and $T=0.7 T_{C}$. The intensity of gray color shows current density in direction indicated by arrows. b) The amplitudes of the first harmonic $\Upsilon_{A}$ (solid line) and the second one $\Upsilon_{B}$ (dashed line) normalized on $2 W \Delta / e \rho_{N} \gamma_{B N}$ versus reduced thickness $\gamma_{B M}$. Inset shows the ratio of harmonics $\left|\Upsilon_{B} / \Upsilon_{A}\right|$ versus $\gamma_{B M}$.

The $I_{F}(\varphi)$ term in (1.34) is the current through one dimensional double barrier SFS structure defined by Eq. (1.17), while $I_{F N}(\varphi)$ is FN-interface term shown in 1.8.4. We provide sufficient smallness and neglect it in the following estimations.

As we discussed above, the larger the relative amplitude of the second harmonic (or the lower the temperature of a junction compare to $T_{C}$ ), the better the conditions for the implementation of a $\varphi$-contact. In the limit $T \ll T_{C}$ we can transform from summation to integration over $\omega$ in (1.35) and calculate numerically the dependence of amplitudes $A$ and $B$

$$
\begin{aligned}
A_{N} & =\frac{2 W \Delta}{e \rho_{N} \gamma_{B N}} \Upsilon_{A}, \\
B_{N} & =\frac{2 W \Delta}{e \rho_{N} \gamma_{B N}} \Upsilon_{B}
\end{aligned}
$$

on suppression parameter $\gamma_{B M}$. The calculated dependencies of functions $\Upsilon_{A}\left(\gamma_{B M}\right)$ and $\left|\Upsilon_{B}\right|\left(\gamma_{B M}\right)$ are presented in Fig.1.9b. It is seen that both $\Upsilon_{A}$ and $\left|\Upsilon_{B}\right|$ increase with increasing of $\gamma_{B M}$ and saturate at $\gamma_{B M} \approx 1$. Inset in Fig.1.9b shows the ratio of the harmonics $\left|\Upsilon_{B} / \Upsilon_{A}\right|$ as a function of $\gamma_{B M}$. It achieves maximum at $\gamma_{B M} \approx 0.64$, thus it 
determines the optimal values of normalized amplitudes of the first $\Upsilon_{A} \approx 0.844$ and the second $\Upsilon_{B} \approx-0.175$ harmonics of the current flowing in the $\mathrm{N}$ layer. It is seen from the inset in Fig.??, that the ratio $\left|\Upsilon_{B} / \Upsilon_{A}\right|$ is slowly decreasing function of $\gamma_{B M}$. Therefore, the estimates given below for $\gamma_{B M}=0.64$ are applicable in a wide parameter range $0.5 \leq \gamma_{B M} \leq 10$.

Taking into account these values, we can write down the condition of $\varphi$-state existence similar to $(1.23)$

$$
\begin{gathered}
\left|\Upsilon_{A}+\frac{1}{\varepsilon} \Psi(L)\right| \leq 2\left|\Upsilon_{B}\right|, \varepsilon=\frac{\sqrt{h} \gamma_{B F}^{2}}{\gamma_{B N}} \frac{\xi_{F} \rho_{F}}{d_{F} \rho_{N}}, \\
\Psi(L)=\exp (-\kappa L) \cos \left(\kappa L+\frac{\pi}{4}\right),
\end{gathered}
$$

with slightly modified dimensionless parameter $\varepsilon$. The wide region of $\varphi$-state still exists if $\varepsilon$ is within the interval

$$
0.123 \lesssim \varepsilon \lesssim 0.298
$$

for $\kappa L$ that satisfies the condition (1.38). As follows from (1.38), interval of $\kappa L$ product gains its maximum length

$$
0.94 \lesssim \kappa L \lesssim 2.72
$$

at $\varepsilon=0.123$. It is seen that these intervals are slightly larger than those given by (1.25) for the ramp type geometry.

Fig.1.10 shows the interval of $\varphi$-state existence, $\Delta L$, in the ideal case of $T \ll T_{C}$, $\gamma_{B M}=0.64$ and $\varepsilon=0.123$. The corresponding set of parameters $d_{N}=0.64 \xi_{N}, d_{F}=1.45 \xi_{N}$, $\gamma_{B N}=1, \gamma_{B F}=1, \xi_{F}=0.1 \xi_{N}, \rho_{N}=\rho_{F}, H=10 T_{C}$ was substituted in (1.17), (1.35). The solid line is a modulus of the first harmonic amplitude, $A$, its normal, $A_{N}$, and ferromagnetic, $A_{F}$, parts are presented by dashed and dash-dotted lines respectively. Finally, the second harmonic amplitude is shown as dash-dot-dotted line. It's clear that $|A|$ is relatively small in the wide region $\Delta L$ and reaches the value of $|2 B|$ only at local maximum. The increased width of $\Delta L$ (see Eqs. (29),(49)) is provided by geometric attributes of RTO type structure.

Let us illustrate the range of nontrivial ground phase $\varphi_{g}$ existence in the structure described in Fig.1.10. The total supercurrent $I_{S}$ is shown on Fig.1.11 as a function of Josephson phase $\varphi$ and electrode spacing $L$. It means that each $L$-section of this $3 \mathrm{D}$ graph is CPR. Solid lines mark the ground state phases at each $L$. In the range of small and large spacing $L$ ground phase is located at $\varphi_{g}=0$. However, in the $\Delta L$-interval CPR becomes 


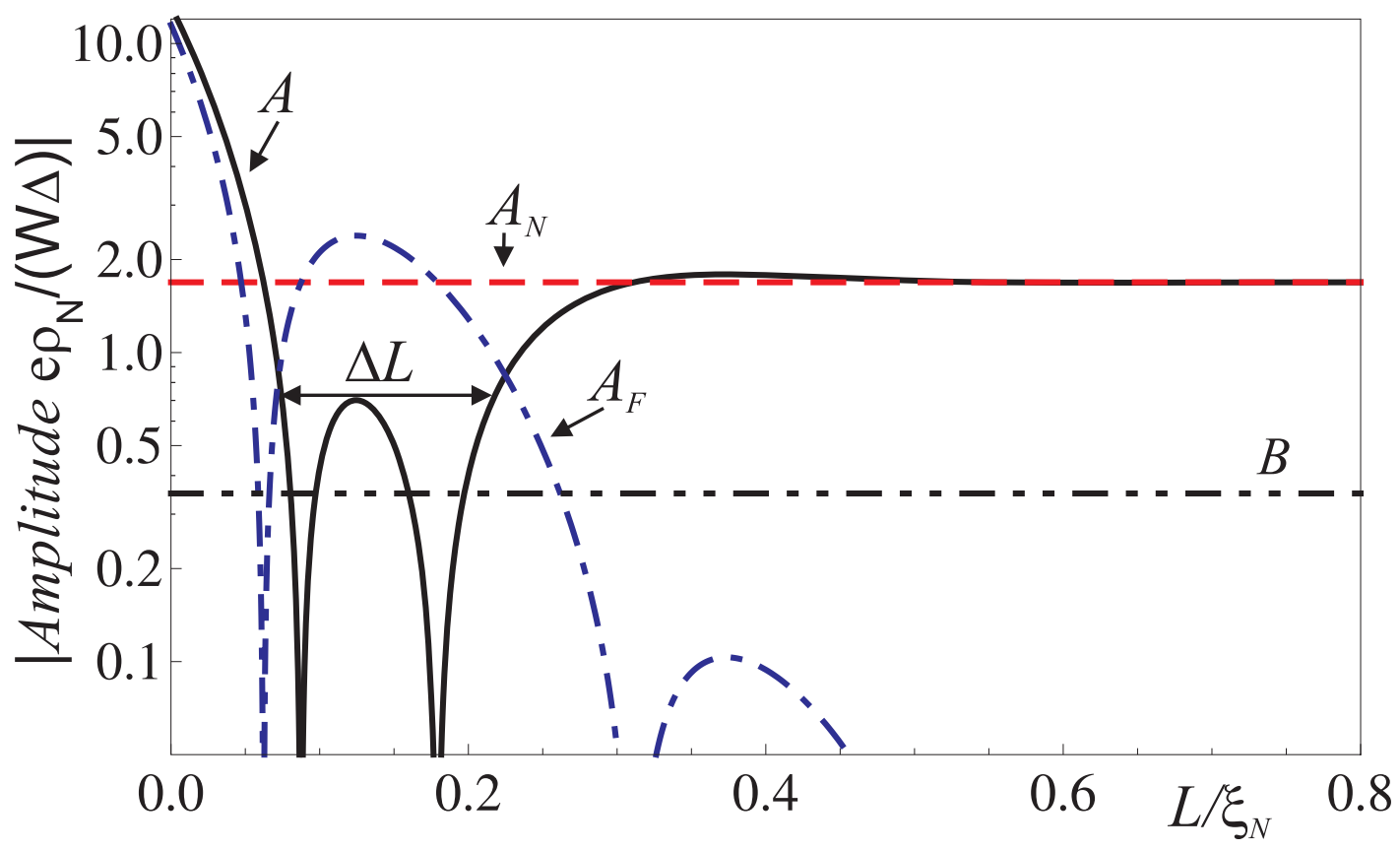

Figure 1.10. The amplitudes of CPR harmonics $A, A_{N}, A_{F}, B$ versus electrode spacing $L$ for RTO structure at $T \ll T_{C}, \gamma_{B M}=0.64$ and $\varepsilon=0.123$. The mark " $\Delta L$ " shows enhanced $\varphi$-state interval.

significantly nonsinusoidal and demands ground phase $\varphi_{g}$ to split and go to $\pi$ from both sides; then $\pi$-state is realized at $\kappa L=\pi / 2$. Clearly, for $\varepsilon \gtrsim 0.123$ the value $\varphi_{g}=\pi$ can not be reached (see Fig.1.11a), while in the case of $\varepsilon \lesssim 0.123$ the prolonged $\pi$-state region is formed (see Fig.1.11c).

\subsection{Discussion}

We have shown that stable $\varphi$-state can be realized in S-NF-S structures with longitudinally oriented NF-bilayers (though $\varphi$-state can not be achieved in conventional SNS and SFS structures). We have discussed the conditions for realization of $\varphi$-state in ramp-type S-NF-S and RTO-type SN-FN-NS geometries.

Let us discuss most favorable conditions for for experimental realization of $\varphi$ junction. We suggest to use Copper as a normal film $\left(\xi_{N} \approx 100 \mathrm{~nm}\right.$ and $\left.\rho=5 * 10^{-8} \Omega \mathrm{m}\right)$ and strongly diluted ferromagnet like FePd or CuNi alloy $\left(\xi_{F} \approx 10 \mathrm{~nm}, H \approx 10 T_{C}\right)$ as the F-layer. We chose $\mathrm{Nb}\left(T_{C} \approx 9 K\right)$ as a superconducting electrode material since it is commonly used in superconducting circuits applications. We also propose to use sufficiently thick normal layer, above the saturation threshold, when N-layer thickness have almost no effect. After substitution of relevant values into (1.39) and (1.40) we arrived at a fairly broad geometrical margins, within which there is a possibility for implementation 

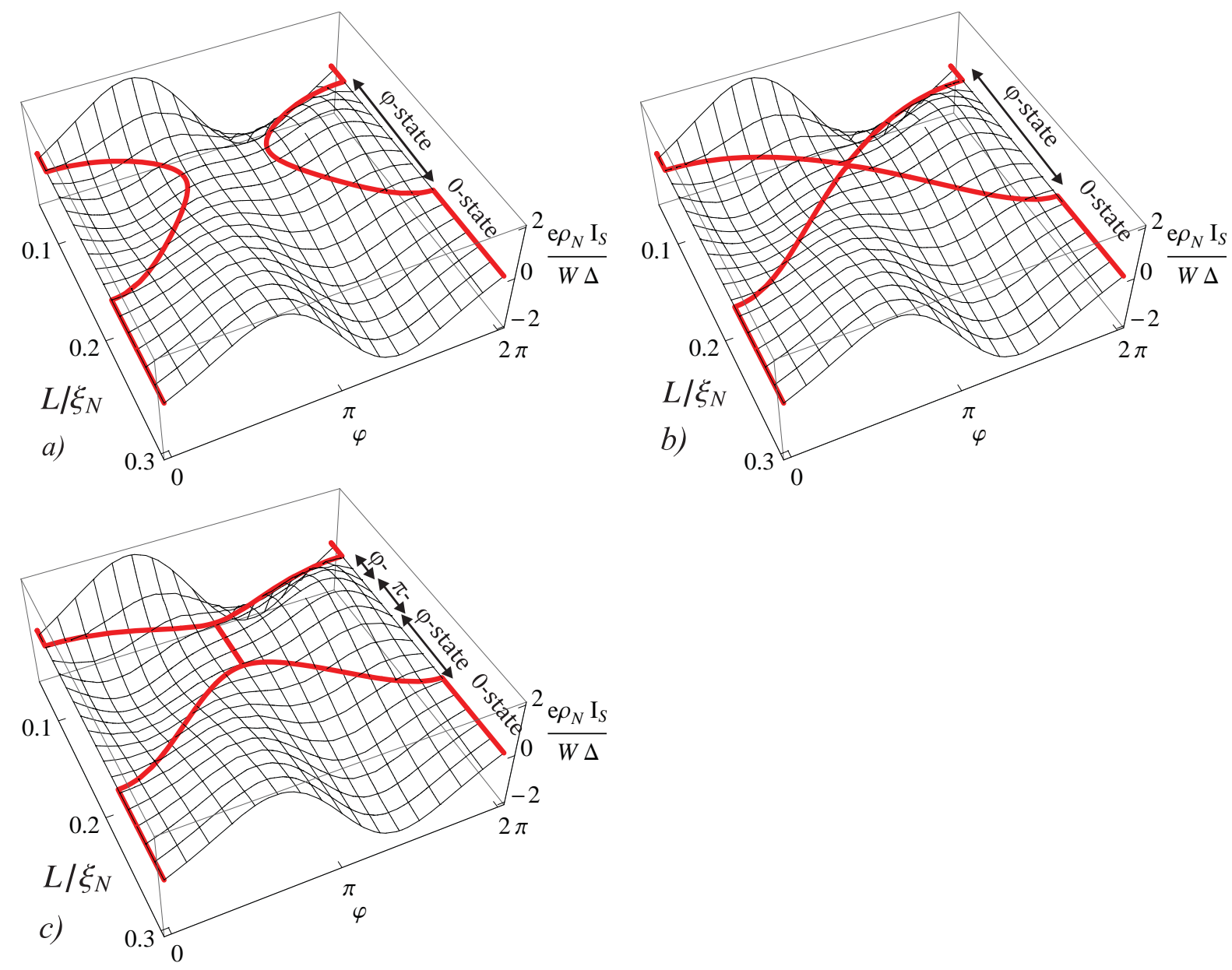

Figure 1.11. The full current $I_{S}$ versus Josephson phase $\varphi$ and electrode spacing $L$ for RTO structure at $T \ll T_{C}, \gamma_{B M}=0.64$ and at different F-layer thickness parameters a) $\varepsilon=0.137, \mathrm{~b}) \varepsilon=0.123$, c) $\varepsilon=0.111$. The lines mark the ground states phase $\varphi_{g}$.

of $\varphi$-junctions

$$
\begin{aligned}
d_{N} & \gtrsim 50 \mathrm{~nm} \\
60 \mathrm{~nm} & \lesssim d_{F} \lesssim 150 \mathrm{~nm} \\
7 \mathrm{~nm} & \lesssim L \lesssim 22 \mathrm{~nm} .
\end{aligned}
$$

Finally, the last out-of-plane geometrical scale is set as $W=140 \mathrm{~nm}$. This value maximizes current and conserves the scale of structure in a range of $100 \mathrm{~nm}$. The magnitude of critical supercurrent in the $\varphi$-state is determined by the second harmonic amplitude $B$

$$
I_{C} \sim B_{N}=\frac{2 W \Delta}{e \rho_{N} \gamma_{B N}} \Upsilon_{B} \approx 1 m A
$$

The spreads of geometrical scales as well as the magnitude of critical current are large enough to be realized experimentally. 
By creating $\varphi$-state in a Josephson junction one can fix certain value of ground phase $\varphi_{g}$. Temperature variation slightly shifts the interval of relevant $0-\pi$ transition and permits one to tune the desired ground state phase. Furthermore, sensitivity of the ground state to an electron distribution function permits $\varphi$-junctions to be applied as small-scale self-biasing one-photon detectors. Moreover, quantum double-well potential is formed at the point of ground state splitting providing necessary condition for quantum bits and quantum detectors. To summarize, Josephson $\varphi$-junctions can be realized using up-to-date technology and may become important basic element in superconducting electronics.

\subsection{Appendix}

\subsubsection{Ramp type junctions. Limit of small $L$}

In the limit of small spacing between $\mathrm{S}$ electrodes

$$
L \ll \min \left\{\xi_{F}, \xi_{N}\right\}
$$

we can neglect nongradient terms in (1.4)

$$
\begin{aligned}
& \frac{\partial}{\partial x}\left(G_{F, N}^{2} \frac{\partial}{\partial x} R_{F, N}\right)+\frac{\partial}{\partial z}\left(G_{F, N}^{2} \frac{\partial}{\partial z} R_{F, N}\right)=0 \\
& \frac{\partial}{\partial x}\left(G_{F, N}^{2} \frac{\partial}{\partial x} U_{F, N}\right)+\frac{\partial}{\partial z}\left(G_{F, N}^{2} \frac{\partial}{\partial z} U_{F, N}\right)=0
\end{aligned}
$$

and introduce four functions

$$
\Phi_{F}=R_{F}+i U_{F}, \Phi_{N}=R_{N}+i U_{N}
$$

where, $i$, is imaginary unit, $R_{F}$ and $R_{N}$ are even function of coordinate $x$, while $U_{F}$ and $U_{N}$ are odd in $x$. Due to the symmetry at $x=0$

$$
\frac{\partial R_{F, N}}{\partial x}=0, U_{F, N}=0
$$

for any coordinate $z$, and it is convenient to rewrite boundary conditions (1.9a), (1.9b) at 
$x=L / 2$ in the form

$$
\begin{aligned}
\gamma_{B N} \xi_{N} \frac{\partial R_{N}}{\partial x} & =\frac{G_{S}}{G_{N}}\left(\Delta \cos (\varphi / 2)-R_{N}\right), \\
\gamma_{B F} \xi_{F} \frac{\partial R_{F}}{\partial x} & =\frac{G_{S}}{G_{F}}\left(\frac{\widetilde{\omega}}{\omega} \Delta \cos (\varphi / 2)-R_{F}\right), \\
\gamma_{B N} \xi_{N} \frac{\partial U_{N}}{\partial x} & =\frac{G_{S}}{G_{N}}\left(\Delta \sin (\varphi / 2)-U_{N}\right), \\
\gamma_{B F} \xi_{F} \frac{\partial U_{F}}{\partial x} & =\frac{G_{S}}{G_{F}}\left(\frac{\widetilde{\omega}}{\omega} \Delta \sin (\varphi / 2)-U_{F}\right) .
\end{aligned}
$$

At NF interface the boundary conditions transforms to:

$$
\begin{aligned}
\gamma_{B F N} \xi_{F} \frac{\partial R_{F}}{\partial z} & =-\frac{G_{N}}{G_{F}}\left(R_{F}-\frac{\widetilde{\omega}}{\omega} R_{N}\right), \\
\gamma_{B N F} \xi_{N} \frac{\partial R_{N}}{\partial z} & =\frac{G_{F}}{G_{N}}\left(R_{N}-\frac{\omega}{\widetilde{\omega}} R_{F}\right), \\
\gamma_{B F N} \xi_{F} \frac{\partial U_{F}}{\partial z} & =-\frac{G_{N}}{G_{F}}\left(U_{F}-\frac{\widetilde{\omega}}{\omega} U_{N}\right), \\
\gamma_{B N F} \xi_{N} \frac{\partial U_{N}}{\partial z} & =\frac{G_{F}}{G_{N}}\left(U_{N}-\frac{\omega}{\widetilde{\omega}} U_{F}\right) .
\end{aligned}
$$

From (1.47) and (1.48a) - (1.49b) it follows that for $\gamma_{B F}$ and $\gamma_{B N}$ within the interval

$$
\frac{L}{\xi_{N}} \ll \gamma_{B N} \ll \frac{\xi_{N}}{L}, \frac{L}{\xi_{1}} \ll \gamma_{B F} \ll \frac{\xi_{1}}{L}
$$

we can neglect $U_{N, F}$ in left hand side of (1.49a), (1.49b). Moreover, in this approximation for any point inside the weak link region $R_{F, N} \gg U_{F, N}$ and the boundary problem (1.44)(1.51b) for functions $R_{F}$ and $R_{N}$ can be solved resulting in

$$
R_{N}=\Delta \cos (\varphi / 2), R_{F}=\frac{\widetilde{\omega}}{\omega} \Delta \cos (\varphi / 2)
$$

and

$$
G_{N}=G_{F}=\frac{\omega}{\sqrt{\omega^{2}+\Delta^{2} \cos ^{2}(\varphi / 2)}}
$$

Therefore under conditions (1.52) both $G_{N}$ and $G_{F}$ are independent on coordinate $x, z$ 
functions and equations for $U_{F, N}$ transform to Laplas equations, which have the solutions

$$
\begin{gathered}
U_{N}=\frac{\Delta \sin (\varphi / 2)}{\gamma_{B N}} \frac{G_{S}}{G_{N}} \frac{x}{\xi_{N}}+ \\
+\sum_{n=1}^{\infty} a_{n} \sin \frac{\pi(2 n+1) x}{L} \cosh \frac{\pi(2 n+1)\left(z-d_{N}-d_{F}\right)}{L}, \\
U_{F}=\frac{\Delta \sin (\varphi / 2)}{\gamma_{B F}} \frac{\widetilde{\omega}}{\omega} \frac{G_{S}}{G_{F}} \frac{x}{\xi_{F}}+ \\
+\frac{\widetilde{\omega}}{\omega} \sum_{n=1}^{\infty} b_{n} \sin \frac{(2 n+1) \pi x}{L} \cosh \frac{\pi(2 n+1) z}{L} .
\end{gathered}
$$

They automatically satisfy the boundary conditions at $z=0$ and $z=d_{N}+d_{F}$, as well as at $x=0$ and $x=L / 2$. To find the integration constants $a_{n}$ and $b_{n}$ we have to substitute (1.55) and (1.56) into (1.51a), (1.51b) and get

$$
\begin{gathered}
a_{n}=-\frac{\Delta \sin (\varphi / 2) G_{S} \Theta \gamma_{B F N} \xi_{F} t_{n}}{G_{N} \beta \cosh \frac{\pi\left(2 n+1 d_{N}\right.}{L}}, t_{n}=\tanh \frac{\pi(2 n+1) d_{N}}{L}, \\
b_{n}=\frac{\Delta \sin (\varphi / 2) G_{S} \Theta \gamma_{B N F} \xi_{N} t_{f}}{G_{N} \beta \cosh \frac{\pi(2 n+1) d_{F}}{L}}, t_{f}=\tanh \frac{\pi(2 n+1) d_{F}}{L},
\end{gathered}
$$

where

$$
\beta=\left(\gamma_{B N F} \xi_{N} \frac{\pi(2 n+1)}{L} t_{n}+1\right) \gamma_{B F N} \xi_{F} t_{f}+\gamma_{B N F} \xi_{N} t_{n}
$$

and

$$
\Theta=\left(\frac{1}{\gamma_{B N} \xi_{N}}-\frac{1}{\gamma_{B F} \xi_{F}}\right) \frac{4 L}{\pi^{2}} \frac{(-1)^{n}}{(2 n+1)^{2}} .
$$

Substitution of (1.55) and (1.56) into expression for the supercurrent (1.5) gives that contributions to the supercurrent across the junction proportional to $a_{n}$ and $b_{n}$ cancel each other and $I_{S}(\varphi)$ equals to the sum

$$
\begin{gathered}
I_{S}(\varphi)=I_{N}(\varphi)+I_{F}(\varphi) \\
\frac{2 e I_{N}(\varphi)}{\pi T W d_{N}}=\frac{1}{\gamma_{B N} \xi_{N} \rho_{N}} \sum_{\omega=-\infty}^{\infty} \frac{\Delta^{2} G_{N} G_{S} \sin (\varphi)}{\omega^{2}} \\
\frac{2 e I_{F}(\varphi)}{\pi T W d_{F}}=\frac{1}{\gamma_{B F} \xi_{F} \rho_{F}} \sum_{\omega=-\infty}^{\infty} \frac{\Delta^{2} G_{F} G_{S} \sin (\varphi)}{\omega^{2}}
\end{gathered}
$$

of the currents, $I_{N}(\varphi)$, and, $I_{F}(\varphi)$, flowing independently across $\mathrm{F}$ and N parts of the weak link. 


\subsubsection{Ramp type junctions. Limit of intermediate $L$}

For intermediate values of spacing between the S electrodes

$$
\xi_{1} \ll L \ll \xi_{N} .
$$

and suppression parameters at SN and SF interfaces belonging to the interval (1.3) the boundary problem (1.4)-(1.9b) can be also solved analytically for sufficiently large suppression parameter $\gamma_{B F N}$. Under these restrictions in the first approximation we can neglect the suppression of superconductivity in the $\mathrm{N}$ film due to proximity with the $\mathrm{F}$ layer and use expressions (1.53) and (1.56) with $a_{n}=0$ as the solution in the $\mathrm{N}$ part of the weak link.

To find $R_{F}$ and $U_{F}$ we have to solve the linear equations

$$
\begin{aligned}
& \xi_{F}^{2} \frac{\partial^{2}}{\partial x^{2}} R_{F}+\xi_{F}^{2} \frac{\partial^{2}}{\partial z^{2}} R_{F}-\widetilde{\Omega} R_{F}=0, \\
& \xi_{F}^{2} \frac{\partial^{2}}{\partial x^{2}} U_{F}+\xi_{F}^{2} \frac{\partial^{2}}{\partial z^{2}} U_{F}-\widetilde{\Omega} U_{F}=0,
\end{aligned}
$$

with the boundary conditions

$$
\begin{aligned}
\gamma_{B F} \xi_{F} \frac{\partial R_{F}}{\partial x} & =G_{S} \frac{\widetilde{\Omega}}{\Omega} \Delta \cos (\varphi / 2), \\
\gamma_{B F} \xi_{F} \frac{\partial U_{F}}{\partial x} & =G_{S} \frac{\widetilde{\Omega}}{\Omega} \Delta \sin (\varphi / 2),
\end{aligned}
$$

at $x=L / 2,0 \leq z \leq d_{F}$ and

$$
\begin{aligned}
\gamma_{B F N} \xi_{F} \frac{\partial R_{F}}{\partial z} & =\frac{\widetilde{\Omega}}{\Omega} G_{N} R_{N}, \\
\gamma_{B F N} \xi_{F} \frac{\partial U_{F}}{\partial z} & =\frac{\widetilde{\Omega}}{\Omega} G_{N} U_{N},
\end{aligned}
$$

at $z=d_{F}, 0 \leq x \leq L / 2 ;\left(\Omega=\omega / \pi T_{C}, \widetilde{\Omega}=\widetilde{\omega} \operatorname{sign}(\omega) / \pi T_{C}\right)$. The boundary problem (1.61)(1.66) must be closed by the conditions (1.7) and (1.47) at free interface of the F film and at the line of junction symmetry, respectively.

Spatial distribution of even in coordinate $x$ part of $\Phi_{F}(x, z)$ can be found in the form of superposition of superconducting correlations induced into F film from superconductors 
and from the N part of weak link

$$
\begin{aligned}
R_{F} & =\frac{\sqrt{\widetilde{\Omega}} G_{S} \Delta \cos (\varphi / 2)}{\Omega \gamma_{B F}} \frac{\cosh \left(\sqrt{\widetilde{\Omega}} \frac{x}{\xi_{F}}\right)}{\sinh \left(\sqrt{\widetilde{\Omega}} \frac{L}{2 \xi_{F}}\right)}+ \\
+ & \frac{\sqrt{\widetilde{\Omega}} G_{N} \Delta \cos (\varphi / 2)}{\Omega \gamma_{B F N}} \frac{\cosh \left(\sqrt{\widetilde{\Omega}} \frac{z}{\bar{\xi}_{F}}\right)}{\sinh \left(\sqrt{\widetilde{\Omega}} \frac{d_{F}}{\xi_{F}}\right)} .
\end{aligned}
$$

Solution for the odd part of $\Phi_{F}(x, z)$ consists of three terms

$$
\begin{gathered}
U_{F}=\frac{\sqrt{\widetilde{\Omega}} G_{S} \Delta \sin (\varphi / 2)}{\Omega \gamma_{B N} \gamma_{B F N}} \frac{x \cosh \left(\sqrt{\widetilde{\Omega}} \frac{z}{\xi_{F}}\right)}{\xi_{N} \sinh \left(\sqrt{\widetilde{\Omega}} \frac{d_{F}}{\xi_{F}}\right)}- \\
-\frac{\widetilde{\Omega}^{3 / 2} G_{S} \Delta \sin (\varphi / 2) \xi_{F}^{2}}{\Omega \gamma_{B N} \xi_{N} \gamma_{B F N} d_{F}} \sum_{n=-\infty}^{\infty} \frac{(-1)^{n} \cos \left(\frac{\pi n z}{d_{F}}\right) \sinh \left(\kappa_{n} \frac{x}{\xi_{F}}\right)}{\kappa_{n}^{3} \cosh \left(\kappa_{n} \frac{L}{2 \xi_{F}}\right)}+ \\
+\frac{\sqrt{\widetilde{\Omega}} G_{S} \Delta \sin (\varphi / 2)}{\Omega \gamma_{B F}} \frac{\sinh \left(\sqrt{\widetilde{\Omega}} \frac{x}{\xi_{F}}\right)}{\cosh \left(\sqrt{\widetilde{\Omega}} \frac{L}{2 \xi_{F}}\right)},
\end{gathered}
$$

where $\kappa_{n}^{2}=\widetilde{\Omega}+\left(\pi n \xi_{F} / d_{F}\right)^{2}$. The first two give the part of $U_{F}$ induced from the $\mathrm{N}$ film, while the last has the well known for SFS junction form [20], [21], [22].

From (1.67) and (1.68) it follows that $R_{-\omega, F}^{*}=R_{\omega, F}$ and $U_{-\omega, F}^{*}=U_{\omega, F}$. Substitution of (1.67) and (1.68) into expression for the supercurrent (1.5) gives that the $I_{S}(\varphi)$ dependence is consists of three terms

$$
I_{S}(\varphi)=I_{N}(\varphi)+I_{F}(\varphi)+I_{F N}(\varphi)
$$

The first is the supercurrent across the N layer. In considered approximation it coincides with the expression given by (1.58). The second term in (1.69) is the supercurrent across SFS double barrier structure in the limit of small transparencies of SF interfaces [59]' [70]

$$
\frac{2 e I_{F}(\varphi)}{\pi T W d_{F}}=\frac{\Delta^{2} \sin (\varphi)}{\gamma_{B F}^{2} \xi_{F} \rho_{F}} \sum_{\omega=-\infty}^{\infty} \frac{G_{S}^{2}}{\omega^{2} \sqrt{\widetilde{\Omega}} \sinh \left(2 q_{L}\right)}
$$

and the last consists of two terms, $I_{F N}(\varphi)=I_{1}(\varphi)+I_{2}(\varphi)$ having different $\varphi$-dependence

$$
\begin{gathered}
\frac{2 e I_{1}(\varphi)}{\pi T W d_{F}}=\frac{\Delta^{2} \sin (\varphi)}{\rho_{F} d_{F}} \frac{\xi_{F}}{\gamma_{B F} \gamma_{B F N} \gamma_{B N} \xi_{N}} \sum_{\omega=-\infty}^{\infty} \frac{G_{S}^{2}}{\widetilde{\Omega}^{2} \omega^{2}} \Psi_{1}, \\
\Psi_{1}=\frac{\sqrt{\widetilde{\Omega}}}{\sinh \left(q_{L}\right)}-\frac{2 \widetilde{\Omega}}{\sinh \left(2 q_{L}\right)}, \\
\frac{2 e I_{2}(\varphi)}{\pi T W d_{F}}=\frac{\Delta^{2} \sin (\varphi)}{\gamma_{B F N} \rho_{F} d_{F}} \sum_{\omega=-\infty}^{\infty} \frac{G_{N} G_{S}}{\omega^{2} \widetilde{\Omega}^{2}}\left(\frac{1}{\gamma_{B N} \gamma_{B F N} \xi_{N}} \Psi_{2}+\frac{\widetilde{\Omega}}{\gamma_{B F} \cosh q_{L}}\right), \\
\Psi_{2}=\frac{d_{F} \widetilde{\Omega}\left(2 q_{d}+\sinh \left(2 q_{d}\right)\right)}{4 q_{d} \sinh { }^{2}\left(q_{d}\right)}-\frac{\widetilde{\Omega} \xi_{F}}{q_{d} \cosh \left(q_{L}\right)}-\sum_{n=1}^{\infty} \frac{2 \widetilde{\Omega}^{3} \xi_{F}}{q_{d} \kappa_{n}^{4} \cosh \left(\frac{L \kappa_{n}}{2 \xi_{F}}\right)},
\end{gathered}
$$


where $q_{d}=d_{F} \sqrt{\widetilde{\Omega}} / \xi_{F}, q_{L}=L \sqrt{\widetilde{\Omega}} / 2 \xi_{F}$. In real experimental situation

$$
\xi_{F} \ll \xi_{N}, d_{F} \gg \xi_{F} .
$$

Under the conditions (1.73) some terms of $I_{F N}(\varphi)$ can be neglected. Still existing expressions of it parts $I_{F N 1}(\varphi)-I_{F N 3}(\varphi)$ simplify to

$$
\begin{gathered}
\frac{2 e I_{F N 1}(\varphi)}{\pi T W d_{F}}=\frac{\Delta^{2} \sin (\varphi)}{\gamma_{B F} \gamma_{B F N} \gamma_{B N} \rho_{F} d_{F}} \frac{\xi_{F}}{\xi_{N}} \sum_{\omega=-\infty}^{\infty} \frac{G_{S}^{2}}{\omega^{2} \widetilde{\Omega}^{2}} \frac{\sqrt{\widetilde{\Omega}}}{\sinh q_{L}}, \\
\frac{2 e I_{F N 2}(\varphi)}{\pi T W d_{F}}=\frac{\Delta^{2} \sin (\varphi)}{2 \gamma_{B N} \gamma_{B F N}^{2} \rho_{F} d_{F}} \sum_{\omega=-\infty}^{\infty} \frac{G_{N} G_{S}}{\omega^{2} \widetilde{\Omega}^{3 / 2}} \frac{\xi_{F}}{\xi_{N}}, \\
\frac{2 e I_{F N 3}(\varphi)}{\pi T W d_{F}}=\frac{\Delta^{2} \sin (\varphi)}{\gamma_{B F N} \gamma_{B F} \rho_{F} d_{F}} \sum_{\omega=-\infty}^{\infty} \frac{G_{N} G_{S}}{\omega^{2} \widetilde{\Omega}} \frac{1}{\cosh q_{L}},
\end{gathered}
$$

\subsubsection{Overlap SN-N-NS junctions}

To calculate critical current of SN-N-NS junctions we consider the most practical case of thin $\mathrm{N}$ film

$$
d_{N} \ll \xi_{N}
$$

and sufficiently large $\gamma_{B F N}$ providing the absence of suppression of superconductivity in $\mathrm{N}$ film due to proximity with $\mathrm{F}$ layer. We will also assume that electrode spacing $L$ is also small

$$
L \ll \xi_{N}
$$

in order to have nonsinusoidal CPR.

Condition (1.77) permits to perform averaging of Usadel equations in $z$-direction in $\mathrm{N}$ film, as it was described in detail in [47], and reduce the problem to the solution of one dimensional equations for $\Phi_{N}=R_{N}+i U_{N}$. The real part of $\Phi_{N}$ is the solution of the boundary problem

$$
\begin{gathered}
\frac{\xi_{N}^{2} \gamma_{B M}}{G_{N}\left(\Omega \gamma_{B M}+G_{S}\right)} \frac{\partial}{\partial x}\left(G_{N}^{2} \frac{\partial R_{N}}{\partial x}\right)-R_{N}=-r \Delta \cos \frac{\varphi}{2}, \frac{L}{2} \leq x \leq \infty, \\
\frac{\xi_{N}^{2}}{\Omega G_{N}} \frac{\partial}{\partial x}\left(G_{N}^{2} \frac{\partial R_{N}}{\partial x}\right)=0,0 \leq x \leq \frac{L}{2}, \\
\frac{\partial R_{N}}{\partial x}=0, \quad x=0, \quad x \rightarrow \infty
\end{gathered}
$$


where $r=G_{S} /\left(\Omega \gamma_{B M}+G_{S}\right), \gamma_{B M}=\gamma_{B N} d_{N} / \xi_{N}, \delta=\Delta / \pi T_{C}$.

From (1.80), (1.81) it follows that at $0 \leq x \leq L / 2$ functions $R_{N}$ are independent on $x$ constants resulting in

$$
\frac{\partial R_{N}}{\partial x}\left(\frac{L}{2}\right)=0
$$

The arising boundary problem $(1.79),(1.81),(1.82)$ is also satisfied by independent on $x$ constants leading to

$$
R_{N}=r \Delta \cos (\varphi / 2), 0 \leq x<\infty
$$

Introducing now new functions, $\theta$

$$
U_{N}=\mu \tan \theta, G_{N}=\frac{\Omega}{\mu} \cos \theta
$$

where $\mu=\sqrt{\Omega^{2}+r^{2} \delta^{2} \cos ^{2}(\varphi / 2)}$, we get

$$
\begin{gathered}
\lambda^{2} \frac{\partial^{2}}{\partial x^{2}} \theta-\sin (\theta-\phi)=0, \quad \frac{L}{2} \leq x<\infty, \\
\frac{\xi_{N}^{2}}{\cos \theta} \frac{\partial^{2}}{\partial x^{2}} \theta=0,0 \leq x \leq \frac{L}{2}, \\
\theta(0)=0, \frac{\partial \theta}{\partial x}=0, \quad x \rightarrow \infty
\end{gathered}
$$

where

$$
\begin{gathered}
\lambda=\xi_{N} \sqrt{\frac{\Omega \gamma_{B M}}{\left(\Omega \gamma_{B M}+G_{S}\right) \sqrt{\Omega^{2}+r^{2} \delta^{2}}}}, \\
\tan \phi=\frac{r \sin (\varphi / 2)}{\mu} .
\end{gathered}
$$

Solution of Eq. (1.86) can be easily found

$$
\theta(x)=\frac{2 x}{L} \theta\left(\frac{L}{2}\right), 0 \leq x \leq \frac{L}{2}
$$

Solution of Eq. (1.85) can be simplified due to existence of the first integral

$$
\frac{\lambda^{2}}{2}\left(\frac{\partial}{\partial x} \theta\right)^{2}+\cos (\theta-\phi)=1
$$

The constant of integration in the right hand side of (1.91) have been found from the boundary condition (1.87), which demands $\theta \rightarrow \phi$ then $x \rightarrow \infty$. Further integration in 
(1.91) for $L / 2 \leq x<\infty$ gives

$$
\theta=\phi+4 \arctan \left(C_{2} \exp \left(-\frac{x-L / 2}{\lambda}\right)\right)
$$

where $C_{2}$ is integration constant, which should be determined from the matching conditions at $x=L / 2$. For $C_{2}$ they give

$$
\left(\phi+4 \arctan \left(C_{2}\right)\right)=-\frac{2 C_{2}}{1+C_{2}^{2}} \frac{L}{\lambda}
$$

Assuming additionally that $\gamma_{B M}$ is not too small, namely that $L \ll \xi_{N} \min \left(1, \sqrt{\gamma_{B M}}\right)$, from (1.93) it is easy to get

$$
C_{2}=-\tan \left(\frac{\phi}{4}-\frac{L}{4 \lambda} \sin \frac{\phi}{2}\right)
$$

resulting in

$$
\theta(x)=\frac{2 x}{\lambda} \sin \frac{\phi}{2}, 0 \leq x \leq \frac{L}{2} .
$$

From (1.95) it follows that in weak link region $|x| \leq L / 2$

$$
U_{N}=\frac{2 x}{\lambda} \mu \sin \frac{\phi}{2}, G_{N}=\frac{\Omega}{\mu},
$$

while under the $\mathrm{S}$ electrode, $L / 2 \leq x<\infty$

$$
\begin{gathered}
U_{N}=\mu \tan (\phi-4 \arctan (u)), \\
u=\tan \left(\frac{\phi}{4}-\frac{L}{4 \lambda} \sin \frac{\phi}{2}\right) \exp \left(-\frac{x-L / 2}{\lambda}\right) .
\end{gathered}
$$

Substitution of (1.83), (1.96) into expression (1.5) for the supercurrent in the N channel results in

$$
\frac{2 e I_{N}(\varphi)}{\pi T W d_{N}}=\frac{2}{\rho_{N} \xi_{N} \sqrt{\gamma_{B M}}} \sum_{\omega=-\infty}^{\infty} \frac{r^{2} \delta^{2} \sin \varphi \sqrt{\left(\Omega \gamma_{B M}+G_{S}\right)}}{\sqrt{2 \Omega \mu^{2}\left(\sqrt{\Omega^{2}+r^{2} \delta^{2}}+\mu\right)}}
$$

\subsubsection{Solution in Ferromagnet Layer of RTO junction}

Spatial distribution of even and odd in coordinate $x$ parts of $\Phi_{F}(x, z)$ can be found in the form of superposition of superconducting correlations induced into F film from superconductors and from the $\mathrm{N}$ part of weak link. It has the same form as in (1.67) and 


$$
\begin{gathered}
R_{F}=\frac{\sqrt{\widetilde{\Omega}} G_{S} \Delta \cos (\varphi / 2)}{\Omega \gamma_{B F}} \frac{\cosh \left(\sqrt{\widetilde{\Omega}} \frac{x}{\xi_{F}}\right)}{\sinh \left(\sqrt{\widetilde{\Omega}} \frac{L}{2 \xi_{F}}\right)}+ \\
+\frac{\sqrt{\widetilde{\Omega}} G_{N} R_{N}}{\Omega \gamma_{B F}} \frac{\cosh \left(\sqrt{\widetilde{\Omega}} \frac{z}{\xi_{F}}\right)}{\sinh \left(\sqrt{\widetilde{\Omega}} \frac{d_{F}}{\xi_{F}}\right)}, \\
U_{F}=\frac{\sqrt{\widetilde{\Omega}} G_{N} U_{N}}{\Omega \gamma_{B F N}} \frac{\cosh \left(\sqrt{\widetilde{\Omega}} \frac{z}{\bar{\xi}_{F}}\right)}{\sinh \left(\sqrt{\widetilde{\Omega}} \frac{d_{F}}{\xi_{F}}\right)}- \\
-\frac{\widetilde{\Omega}^{3 / 2} \xi_{F}^{2} G_{N}\left(U_{N} / x\right)}{\Omega \gamma_{B F N} d_{F}} \sum_{n=-\infty}^{\infty} \frac{(-1)^{n} \cos \left(\frac{\pi n z}{d_{F}}\right) \sinh \left(\kappa_{n} \frac{x}{\xi_{F}}\right)}{\kappa_{n}^{3} \cosh \left(\kappa_{n} \frac{L}{2 \xi_{F}}\right)}+ \\
+\frac{\sqrt{\widetilde{\Omega}} G_{S} \delta \sin (\varphi / 2)}{\Omega \gamma_{B F}} \frac{\sinh \left(\sqrt{\widetilde{\Omega}} \frac{x}{\xi_{F}}\right)}{\cosh \left(\sqrt{\widetilde{\Omega}} \frac{L}{2 \xi_{F}}\right)},
\end{gathered}
$$

with the functions $R_{N}, G_{N}$, and $U_{N}$ defined by equations followed from the solution of the boundary problem in the $\mathrm{N}$ layer described in Appendix 1.8.3.

$$
\begin{gathered}
R_{N}=r \Delta \cos (\varphi / 2), \quad G_{N}=\frac{\Omega}{\sqrt{\Omega^{2}+r^{2} \delta^{2} \cos ^{2}(\varphi / 2)}}, \\
U_{N}=\alpha \Delta \sin (\varphi / 2) \frac{G_{S}}{G_{N}} \frac{x}{\xi_{N}} \\
\alpha=\frac{2 \sqrt{\Omega^{2}+\delta^{2}}}{\sqrt{2\left(\sqrt{\left.\Omega^{2}+r^{2} \delta^{2}+\mu\right)}\right.} \frac{r}{\sqrt{1-r}}} .
\end{gathered}
$$

Substitution of (1.99)-(1.102) into expression (1.5) gives that supercurrent across F layer in RTO junction consists of the sum of $I_{F}(\varphi)$ and $I_{F N}(\varphi)$, where $I_{F}(\varphi)$ is the current through one dimensional double barrier SFS structure defined by Eq. (1.70), while $I_{F N}(\varphi)=I_{1}(\varphi)+$ $I_{2}(\varphi)$ has the form

$$
\begin{gathered}
\frac{2 e I_{1}(\varphi)}{\pi T W d_{F}}=\frac{\Delta^{2} \sin (\varphi)}{\rho_{F} d_{F}} \frac{\xi_{F}}{\gamma_{B F} \gamma_{B F N} \xi_{N}} \sum_{\omega=-\infty}^{\infty} \frac{\alpha G_{S}^{2}}{\widetilde{\Omega}^{2} \omega^{2}} \Psi_{1}, \\
\Psi_{1}=\frac{\sqrt{\widetilde{\Omega}}}{\sinh \left(q_{L}\right)}-\frac{2 \widetilde{\Omega}}{\sinh \left(2 q_{L}\right)}, \\
\frac{2 e I_{2}(\varphi)}{\pi T W d_{F}}=\frac{\Delta^{2} \sin (\varphi)}{\rho_{F} d_{F}} \frac{1}{\gamma_{B F N}} \sum_{\omega=-\infty}^{\infty} \frac{r G_{N} G_{S}}{\omega^{2} \widetilde{\Omega}^{2}}\left(\frac{\alpha}{\gamma_{B F N} \xi_{N}} \Psi_{2}+\frac{\widetilde{\Omega}}{\gamma_{B F} \cosh q_{L}}\right), \\
\Psi_{2}=\frac{d_{F} \widetilde{\Omega}\left(2 q_{d}+\sinh \left(2 q_{d}\right)\right)}{4 q_{d} \sinh ^{2}\left(q_{d}\right)}-\frac{\widetilde{\Omega} \xi_{F}}{q_{d} \cosh \left(q_{L}\right)}-\sum_{n=1}^{\infty} \frac{2 \widetilde{\Omega}^{3} \xi_{F}}{q_{d} \kappa_{n}^{4} \cosh \left(\frac{L \kappa_{n}}{2 \xi_{F}}\right)} .
\end{gathered}
$$

Application of conditions (1.73) allows to neglect some terms in $I_{F N}(\varphi)=I_{F N 1}(\varphi)+$ $I_{F N 2}(\varphi)+I_{F N 3}(\varphi)$ and to simplify remaining terms, leading to the following expressions:

$$
\begin{gathered}
\frac{2 e I_{F N 1}(\varphi)}{\pi T W d_{F}}=\frac{\Delta^{2} \sin (\varphi)}{\gamma_{B F} \gamma_{B F N} \rho_{F} d_{F}} \frac{\xi_{F}}{\xi_{N}} \sum_{\omega=-\infty}^{\infty} \frac{\alpha G_{S}^{2}}{\omega^{2} \widetilde{\Omega}^{2}} \frac{\sqrt{\widetilde{\Omega}}}{\sinh q_{L}}, \\
\frac{2 e I_{F N 2}(\varphi)}{\pi T W d_{F}}=\frac{\Delta^{2} \sin (\varphi)}{2 \gamma_{B F N}^{2} \rho_{F} d_{F}} \sum_{\omega=-\infty}^{\infty} \frac{r \alpha G_{N} G_{S}}{\omega^{2} \widetilde{\Omega}^{3 / 2}} \frac{\xi_{F}}{\xi_{N}}
\end{gathered}
$$




$$
\frac{2 e I_{F N 3}(\varphi)}{\pi T W d_{F}}=\frac{\Delta^{2} \sin (\varphi)}{\gamma_{B F N} \gamma_{B F} \rho_{F} d_{F}} \sum_{\omega=-\infty}^{\infty} \frac{r G_{N} G_{S}}{\omega^{2} \widetilde{\Omega}} \frac{1}{\cosh q_{L}}
$$




\section{Bibliography}

[1] K.K. Likharev, Rev. Mod. Phys. 51, 101 (1979).

[2] A. A. Golubov, M. Yu. Kupriyanov, E. Il'ichev, Rev. Mod. Phys. 76, 411 (2004).

[3] A. I. Buzdin, Rev. Mod. Phys. 77, 935 (2005).

[4] F. S. Bergeret, A. F. Volkov, K. B. Efetov, Rev. Mod. Phys. 77, 1321 (2005).

[5] V. V. Ryazanov, V. A. Oboznov, A. Yu. Rusanov, A. V. Veretennikov, A. A. Golubov, and J. Aarts, Phys. Rev. Lett. 86, 2427 (2001).

[6] S. M. Frolov, D. J. Van Harlingen, V. A. Oboznov, V. V. Bolginov, and V. V. Ryazanov, Phys. Rev. B 70, 144505 (2004);

[7] T. Kontos, M. Aprili, J. Lesueur, F. Genet, B. Stephanidis, and R. Boursier, Phys. Rev. Lett. 89, 137007 (2002).

[8] H. Sellier, C. Baraduc, F. Lefloch, and R. Calemczuck, Phys. Rev. B 68, 054531 (2003).

[9] Y. Blum, A. Tsukernik, M. Karpovski, and A. Palevski, Phys. Rev. B 70, 214501 (2004).

[10] C. Surgers, T. Hoss, C. Schonenberger, C. Strunk, J. Magn. Magn. Mater. 240, 598 (2002).

[11] C. Bell, R. Loloee, G. Burnell, and M. G. Blamire Phys. Rev. B 71, 180501 (R) (2005).

[12] S. M. Frolov, D. J. Van Harlingen, V. V. Bolginov, V. A. Oboznov, and V. V. Ryazanov, Phys. Rev. B 74, 020503 (2006).

[13] V. A. Oboznov, V. V. Bol'ginov, A. K. Feofanov, V. V. Ryazanov, and A. I. Buzdin, Phys. Rev. Lett. 96, 197003 (2006).

[14] V. Shelukhin, A. Tsukernik, M. Karpovski, Y. Blum, K. B. Efetov, A. F. Volkov, T. Champel, M. Eschrig, T. Lofwander, G. Schon, and A. Palevski, Physical Review B 73, 174506 (2006). 
[15] M. Weides, K. Tillmann, and H. Kohlstedt, Physica C 437-438, 349 (2006).

[16] M. Weides, M. Kemmler, H. Kohlstedt, A. Buzdin, E. Goldobin, D. Koelle, R. Kleiner, Appl. Phys. Lett. 89, 122511 (2006).

[17] M. Weides, M. Kemmler, H. Kohlstedt, R. Waser, D. Koelle, R. Kleiner, and E. Goldobin Physical Review Letters 97247001 (2006).

[18] J. Pfeiffer, M. Kemmler, D. Koelle, R. Kleiner, E. Goldobin, M. Weides, A. K. Feofanov, J. Lisenfeld, and A. V. Ustinov, Physical Review B 77, 214506 (2008).

[19] H. Sellier, C. Baraduc, F. Lefloch, and R. Calemczuck, Phys. Rev. Lett. 92, 257005 (2004).

[20] F. Born, M. Siegel, E. K. Hollmann, H. Braak, A. A. Golubov, D. Yu. Gusakova, and M. Yu. Kupriyanov, Phys. Rev. B. 74, 140501 (2006).

[21] J. W. A. Robinson, S. Piano, G. Burnell, C. Bell, and M. G. Blamire, Phys. Rev. Lett. 97, 177003 (2006).

[22] S. Piano, J. W.A. Robinson, G. Burnell, M. G. Blamire The European Physical Journal B 58, $123(2007)$.

[23] J. W. Robinson, S. Piano, G. Burnell, C. Bell, and M. G. Blamire Physical Review B 76, $094522(2007)$.

[24] R. S. Keizer, S. T. B. Goennenwein, T. M. Klapwijk, G. Miao, G. Xiao, A. Gupta, Nature 439, 825 (2006).

[25] T. S. Khaire, M. A. Khasawneh, W. P. Pratt, Jr., and N. O. Birge, Phys. Rev. Lett. 104, $137002(2010)$.

[26] J. W. A. Robinson, J. D. S. Witt, and M. G. Blamire, Science 329, 59 (2010).

[27] J.Wang, M. Singh, M. Tian, N. Kumar, B. Liu, C. Shi, J. K. Jain, N. Samarth, T. E. Mallouk and M. H. W. Chan, Nat. Phys. 6, 389 (2010).

[28] M. S. Anwar, F. Czeschka, M. Hesselberth, M. Porcu, and J. Aarts, Phys. Rev. B 82, 100501(R) (2010). 
[29] M. S. Anwar, M. Veldhorst, A. Brinkman, and J. Aarts, Appl. Phys.Lett. 100, 052602 (2012).

[30] T. Ortlepp, Ariando, O. Mielke, C. J. M. Verwijs, K. F. K. Foo, H. Rogalla, F. H. Uhlmann, and H. Hilgenkamp, Science 312, 1495 (2006).

[31] A.K. Feofanov, V.A. Oboznov, V.V. Bol'ginov, et. al., Nature Physics 6, 593 (2010).

[32] A. V. Ustinov and V. K. Kaplunenko, J. Appl. Phys. 94, 5405 (2003).

[33] R.G. Mints, Phys. Rev. B 57, R3221 (1998).

[34] A. Buzdin and A. E. Koshelev, Phys. Rev. B 67, 220504(R) (2003).

[35] N. G. Pugach, E. Goldobin, R. Kleiner, and D. Koelle, Phys. Rev. B 81, 104513 (2010).

[36] M.Yu. Kupriyanov, A.A. Golubov, M. Siegel, Proceedings of the SPIE, 6260, 62600S-1 (2006).

[37] E. Goldobin, D. Koelle, R. Kleiner, and R.G. Mints, Phys. Rev. Lett, 107, 227001 (2011); H. Sickinger, A. Lipman, M Weides, R.G. Mints, H. Kohlstedt, D. Koelle, R. Kleiner, and E. Goldobin, arXiv 1207.3013.

[38] E. Goldobin, D. Koelle, R. Kleiner, and A. Buzdin, Phys. Rev. B, 76, 224523 (2007).

[39] N. V. Klenov, N. G.Pugach, A. V.Sharafiev, S. V.Bakurskiy, V. K.Kornev, Physics of Solid State, 52, 2246 (2010).

[40] A. S. Vasenko, A. A. Golubov, M. Yu. Kupriyanov, and M. Weides, Phys.Rev.B, 77 134507 (2008).

[41] A. S. Vasenko, S. Kawabata, A. A. Golubov, M. Yu. Kupriyanov, C. Lacroix, F. W. J. Hekking, Phys. Rev. B 84024524 (2011).

[42] F. Konschelle, J. Cayssol, A.I. Buzdin, Phys. Rev. B 78, 134505 (2008).

[43] M. Houzet, V. Vinokur, and F. Pistolesi, PRB 72, 220506 (2005)

[44] T. Yu. Karminskaya and M. Yu. Kupriyanov, Pis'ma Zh. Eksp. Teor. Fiz. 85, 343 (2007) [JETP Lett. 85, 286 (2007)]. 
[45] T. Yu. Karminskaya and M. Yu. Kupriyanov, Pis'ma Zh. Eksp. Teor. Fiz. 85, 343 (2007) [JETP Lett. 86, 61 (2007)].

[46] T. Yu. Karminskaya, M. Yu. Kupriyanov, and A. A. Golubov, Pis'ma Zh.Eksp. Teor. Fiz. 87, 657 (2008) [JETP Lett., 87, 570 (2008)].

[47] Karminskaya T. Yu., Golubov A. A., Kupriyanov M. Yu., Sidorenko A. S., Phys.Rev.B 79, 214509 (2009).

[48] T. Yu. Karminskaya, A. A. Golubov, M. Yu. Kupriyanov, and A. S. Sidorenko Phys. Rev. B 81, 214518 (2010).

[49] S. V. Bakurskiy, N. V. Klenov, T. Yu. Karminskaya, M. Yu. Kupriyanov and V. K. Kornev, Solid State Phenomena, 190, 401 (2012).

[50] F. S. Bergeret, A. F. Volkov, and K. B. Efetov, Phys. Rev. Lett. 86, 3140 (2001).

[51] Ya. V. Fominov, N. M. Chtchelkatchev, and A. A. Golubov, Phys. Rev. B 66, 014507 (2002).

[52] A. Furusaki and M. Tsukada, Solid State Commun. 78, 299 (1991).

[53] A. Furusaki and M. Tsukada, Phys. Rev. B 43, 10164 (1991).

[54] Z. G.Ivanov, M. Yu. Kupriyanov, K. K. Likharev, S. V. Meriakri, and O. V. Snigirev, Fiz. Nizk. Temp. 7, 560 (1981). [Sov. J. Low Temp. Phys. 7, 274 (1981)]

[55] A. A.Zubkov, and M. Yu. Kupriyanov, Fiz. Nizk. Temp. 9, 548 (1983) [Sov. J. Low Temp. Phys. 9, 279 (1983)].

[56] M. Yu. Kupriyanov, Pis'ma Zh. Eksp. Teor. Fiz. 56, 414 (1992). [JETP Lett. 56, 399 (1992)].

[57] Demler, E. A., Arnold G. B., and Beasley M. R., Phys. Rev. B 55, 15174 (1997).

[58] K. D. Usadel, Phys. Rev. Lett. 25, 507 (1970).

[59] M. Yu. Kuprianov and V. F. Lukichev, Sov. Phys. JETP 67, 1163 (1988) [Zh. Eksp. Teor. Fiz. 94, 139 (1988)].

[60] N. M. Chtchelkatchev and I. S. Burmistrov, Phys. Rev. B 68, 140501(R) (2003). 
[61] I. S. Burmistrov and N. M. Chtchelkatchev, Phys. Rev. B 72, 144520 (2005).

[62] T. Champel and M. Eschrig, PRB 72, 054523 (2005).

[63] M. Houzet and A. I. Buzdin, Phys. Rev. B 74, 214507 (2006).

[64] M. A. Maleki and M. Zareyan Physical Review B 74, 144512 (2006).

[65] Y. V. Fominov, A. F. Volkov, and K. B. Efetov, Phys. Rev. B 75, 104509 (2007).

[66] A. F. Volkov and A. Anishchanka, Phys. Rev. B 71, 024501 (2005).

[67] A.F. Volkov, K.B. Efetov Phys Rev B 78, 024519 (2008).

[68] B. Crouzy, S. Tollis, D. A. Ivanov, Phys. Rev. B 76, 134502 (2007).

[69] A. A. Golubov, M. Yu. Kupriyanov, and Ya. V. Fominov, JETP Lett. 75, 709 (2002) [Pisma v ZhETF 75, 588 (2002)].

[70] A. Buzdin, JETP Lett. 78, 1073 (2003) [Pisma v ZhETF 78, 1073 (2003)].

[71] M. Yu.Kupriyanov, V. F. Lukichev, A. A. Orlikovskii, Mikroelektronika 15, 328 (1986) [Soviet Microelectronics 15, 185 (1986)]. 


\section{Chapter 2}

\section{Theory of supercurrent transport in SIsFS Josephson junctions}

\section{$2.1 \quad$ Introduction}

Josephson structures with a ferromagnetic layer became a very active field of research because of the interplay between superconducting and magnetic order in a ferromagnet, leading to a variety of new effects including the realization of a $\pi$-state with phase difference $\pi$ in the ground state of a junction, as well as long-range Josephson coupling due to generation of an odd-frequency triplet order parameter [20-22].

Further interest to Josephson junctions with magnetic barrier is due to emerging possibilities for their practical use as elements of a superconducting memory [1] ${ }^{-}[12]$, onchip $\pi$ - phase shifters for self-biasing various electronic quantum and classical circuits [13] ${ }^{-}$ [16], and $\varphi$ - batteries, structures having in the ground state a phase difference $\varphi_{g}=\varphi$ $(0<|\varphi|<\pi)$ between superconducting electrodes [17-25]. In standard experimental implementations SFS Josephson junctions are sandwich-type structures [26] ${ }^{-}[27]$. The characteristic voltage $V_{C}=J_{C} R_{N}$ ( $J_{C}$ is the critical current of the junction and $R_{N}$ is the resistance in the normal state) of these SFS devices is typically quite low, which limits their practical applications. In SIFS structures $[28]^{-}[32]$ containing an additional insulating barrier I, the $J_{C} R_{N}$ product in the 0 -state is increased [17], but in the $\pi$-state $V_{C}$ is still too small $[24,34]$ due to strong suppression of the superconducting correlations in the ferromagnetic layer.

Recently, a different type of magnetic Josepshon junction was realized experimentally, containing two superconducting layers with IsF a complex weak link consisting of a superconducting film s, a metallic ferromagnet F, and an insulating barrier I. [12, 15-17]. This structure represents a connection of an SIs tunnel junction and an sFS contact in series. Properties of SIsFS structures are controlled by the thickness of the s layer $d_{s}$ and by the relation between the critical currents $J_{C S I s}$ and $J_{C s F S}$ of their SIs and sFS parts, respectively. If the thickness of the s-layer $d_{s}$ is much larger than its coherence length $\xi_{S}$ and $J_{C S I s} \ll J_{C s F S}$, then the characteristic voltage of an SIsFS device is determined by 
its SIs part and may reach its maximum corresponding to a standard SIS junction. At the same time, the phase difference $\varphi$ in the ground state of an SIsFS junction is controlled by its sFS part. As a result, both 0 and $\pi$ states can be achieved depending on the thickness of the F layer. This opens the possibility of realizing controllable $\pi$ junctions having a large $J_{C} R_{N}$ product. At the same time, when placed in an external magnetic field $H_{\text {ext }}$, the SIsFS structure behaves as a single junction, since $d_{s}$ is typically too thin to screen $H_{\text {ext }}$. This provides the possibility of switching $J_{C}$ by an external field.

a)
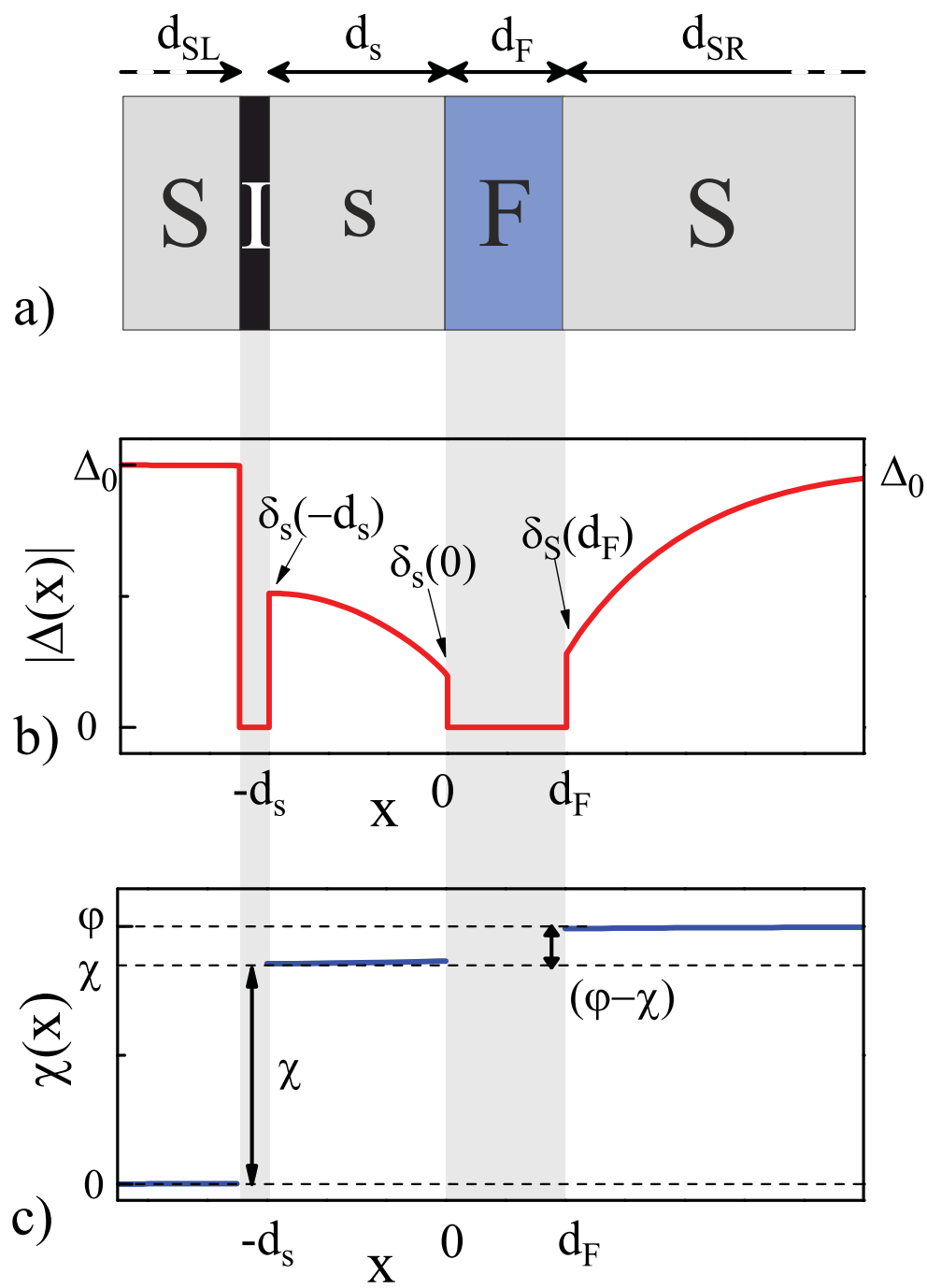

Figure 2.1. a) Schematic design of SIsFS Josephson junction. b), c) Typical distribution of amplitude $|\Delta(x)|$ and phase difference $\chi(x)$ of the pair potential along the structure.

The purpose of this paper is to develop a microscopic theory providing the depen- 
dence of the characteristic voltage on temperature $T$, exchange energy $H$ in the ferromagnet, transport properties of FS and sF interfaces, and the thicknesses of the s and F layers. Special attention will be given to determining the current-phase relation (CPR) between the supercurrent $J_{S}$ and the phase difference $\varphi$ across the structure.

\subsection{Model of SIsFS Josephson device}

We consider the multilayered structure presented in Fig.2.1a. It consists of two superconducting electrodes separated by a complex interlayer including a tunnel barrier I, intermediate superconducting $\mathrm{s}$ and ferromagnetic $\mathrm{F}$ films. We assume that the conditions of the dirty limit are fulfilled for all materials in the structure. In order to simplify the problem, we also assume that all superconducting films are identical and can be described by a single critical temperature $T_{C}$ and coherence length $\xi_{S}$. Transport properties of both $\mathrm{sF}$ and FS interfaces are also assumed identical and are characterized by the interface parameters

$$
\gamma=\frac{\rho_{S} \xi_{S}}{\rho_{F} \xi_{F}}, \quad \gamma_{B}=\frac{R_{B F} \mathcal{A}_{B}}{\rho_{F} \xi_{F}} .
$$

Here $R_{B F}$ and $\mathcal{A}_{B}$ are the resistance and area of the sF and FS interfaces, $\xi_{S}$ and $\xi_{F}$ are the decay lengths of $\mathrm{S}$ and $\mathrm{F}$ materials, and $\rho_{S}$ and $\rho_{F}$ are their resistivities.

Under the above conditions the problem of calculation of the critical current in the SIsFS structure reduces to solution of the set of Usadel equations [19]. For the S layers these equations have the form [20-22]

$$
\begin{gathered}
\frac{\xi_{S}^{2}}{\Omega G_{m}} \frac{d}{d x}\left(G_{m}^{2} \frac{d}{d x} \Phi_{m}\right)-\Phi_{m}=-\Delta_{m}, G_{m}=\frac{\Omega}{\sqrt{\Omega^{2}+\Phi_{m} \Phi_{m}^{*}}} \\
\Delta_{m} \ln \frac{T}{T_{C}}+\frac{T}{T_{C}} \sum_{\omega=-\infty}^{\infty}\left(\frac{\Delta_{m}}{|\Omega|}-\frac{\Phi_{m} G_{m}}{\Omega}\right)=0
\end{gathered}
$$

where $m=S$ for $x \leq-d_{s}$ and $x \geq d_{F} ; m=s$ in the interval $-d_{s} \leq x \leq 0$. In the F film $\left(0 \leq x \leq d_{F}\right)$ the equation is

$$
\xi_{F}^{2} \frac{d}{d x}\left(G_{F}^{2} \frac{d}{d x} \Phi_{F}\right)-\widetilde{\Omega} \Phi_{F} G_{F}=0 .
$$

Here $\Omega=T(2 n+1) / T_{C}$ are Matsubara frequencies normalized to $\pi T_{C}, \widetilde{\Omega}=\Omega+i H / \pi T_{C}$, $G_{F}=\widetilde{\Omega} /\left(\widetilde{\Omega}^{2}+\Phi_{F, \omega} \Phi_{F,-\omega}^{*}\right)^{1 / 2}, H$ is the exchange energy, $\xi_{S, F}^{2}=\left(D_{S, F} / 2 \pi T_{C}\right)$ and $D_{S, F}$, are the diffusion coefficients in the $\mathrm{S}$ and $\mathrm{F}$ metals, respectively. The pair potential $\Delta_{m}$ 
and the Usadel functions $\Phi_{m}$ and $\Phi_{F}$ in (2.2) - (2.4) are also normalized to $\pi T_{C}$. To write equations (2.2) - (2.4), we have chosen the $x$ axis in the direction perpendicular to the SI, FS and $\mathrm{sF}$ interfaces and put the origin at the sF interface. Equations (2.2)-(2.4) must be supplemented by the boundary conditions [23]. At $x=-d_{s}$ they can be written as

$$
\begin{aligned}
G_{S}^{2} \frac{d}{d x} \Phi_{S} & =G_{s}^{2} \frac{d}{d x} \Phi_{s} \\
\gamma_{B I} \xi_{S} G_{s} \frac{d}{d x} \Phi_{s} & =-G_{S}\left(\Phi_{S}-\Phi_{s}\right),
\end{aligned}
$$

where $\gamma_{B I}=R_{B I} \mathcal{A}_{B} / \rho_{S} \xi_{S}$, and $R_{B I}$ and $\mathcal{A}_{B}$ are the resistance and area of the SI interface. At $x=0$ the boundary conditions are

$$
\begin{gathered}
\frac{\xi_{S}}{\Omega} G_{s}^{2} \frac{d}{d x} \Phi_{s}=\gamma \frac{\xi_{F}}{\widetilde{\Omega}} G_{F}^{2} \frac{d}{d x} \Phi_{F}, \\
\gamma_{B} \xi_{F} G_{F} \frac{d}{d x} \Phi_{F}=-G_{s}\left(\frac{\widetilde{\Omega}}{\Omega} \Phi_{s}-\Phi_{F}\right)
\end{gathered}
$$

and at $x=d_{F}$ they have the form

$$
\begin{gathered}
\frac{\xi_{S}}{\Omega} G_{S}^{2} \frac{d}{d x} \Phi_{S}=\gamma \frac{\xi_{F}}{\widetilde{\Omega}} G_{S}^{2} \frac{d}{d x} \Phi_{F} \\
\gamma_{B} \xi_{F} G_{F} \frac{d}{d x} \Phi_{F}=G_{S}\left(\frac{\widetilde{\Omega}}{\Omega} \Phi_{S}-\Phi_{F}\right),
\end{gathered}
$$

Far from the interfaces the solution should cross over to a uniform current-carrying superconducting state $[37]^{-}[39]$

$$
\begin{gathered}
\Phi_{S}(\mp \infty)=\Phi_{\infty} \exp \left\{i\left(\chi(\mp \infty)-u x / \xi_{S}\right)\right\}, \\
\Delta_{S}(\mp \infty)=\Delta_{0} \exp \left\{i\left(\chi(\mp \infty)-u x / \xi_{S}\right)\right\}, \\
\Phi_{\infty}=\frac{\Delta_{0}}{1+u^{2} / \sqrt{\Omega^{2}+\left|\Phi_{S}\right|^{2}}}
\end{gathered}
$$

resulting in an order parameter phase difference across the structure equal to

$$
\varphi=\varphi(\infty)-2 u x / \xi_{S}, \varphi(\infty)=\chi(\infty)-\chi(-\infty)
$$

Here $\varphi(\infty)$ is the asymptotic phase difference across the junction, $\Delta_{0}$ is the modulus of order parameters far from the boundaries of the structure at a given temperature, $u=2 m v_{s} \xi_{S}$, $m$ is the electron mass and $v_{s}$ is the superfluid velocity. Note that since the boundary conditions (2.5) and (2.6) include the Matsubara frequency $\Omega$, the phases of the functions 
$\Phi_{S}$ depend on $\Omega$ and are different from the phases of the pair potential $\Delta_{S}$ at the FS interfaces $\chi\left(d_{F}\right)$ and $\chi(0)$. Therefore it is the value $\varphi(\infty)$ rather than $\varphi=\chi\left(d_{F}\right)-\chi(0)$, that can be measured experimentally by using a scheme compensating the linear in $x$ part in Eq. (2.11).

The boundary problem (2.2)-(2.11) can be solved numerically making use of (2.8) and (2.10). The accuracy of the calculations can be monitored by the equality of the currents $J_{S}$

$$
\frac{2 e J_{S}(\varphi)}{\pi T \mathcal{A}_{B}}=\sum_{\omega=-\infty}^{\infty} \frac{i G_{m, \omega}^{2}}{\rho_{m} \widetilde{\Omega}^{2}}\left[\Phi_{m, \omega} \frac{\partial \Phi_{m,-\omega}^{*}}{\partial x}-\Phi_{m,-\omega}^{*} \frac{\partial \Phi_{m, \omega}}{\partial x}\right],
$$

calculated at the SI and FS interfaces and in the electrodes.

In the further analysis carried out below we limit ourselves to the consideration of the most relevant case of a low-transparency tunnel barrier at the SI interface.

$$
\gamma_{B I} \gg 1
$$

In this approximation, the junction resistance $R_{N}$ is fully determined by the barrier resistance $R_{B I}$. Furthermore the current flowing through the electrodes can lead to the suppression of superconductivity only in the vicinity of $\mathrm{sF}$ and FS interfaces. That means, up to terms of the order of $\gamma_{B I}^{-1}$ we can neglect the effects of suppression of superconductivity in the region $x \leq-d_{s}$ and write the solution in the form

$$
\Phi_{S}(x)=\Delta_{S}(x)=\Delta_{0}
$$

Here without any loss of generality we put $\chi(-\infty)=\chi\left(-d_{s}-0\right)=0$ (see Fig. 2.1c).

Substitution of (2.14) into the boundary conditions (2.5) gives

$$
\gamma_{B I} \xi_{S} G_{s} \frac{d}{d x} \Phi_{s}=-\frac{\Omega}{\sqrt{\Omega^{2}+\Delta_{0}^{2}}}\left(\Delta_{0}-\Phi_{s}\right) .
$$

Further simplifications are possible in several limiting cases.

\subsection{The high temperature limit $T \approx T_{C}$}

In the vicinity of the critical temperature the Usadel equations in the $\mathrm{F}$ layer can be linearized. Writing down their solution in analytical form and using the boundary conditions (2.6) and (2.7) on sF and FS interfaces we can reduce the problem to the solution of the Ginzburg-Landau (GL) equations in the s and S layers. We limit our analysis by considering 
the most interesting case when the following condition is fulfilled:

$$
\Gamma_{B I}=\frac{\gamma_{B I} \xi_{S}}{\xi_{S}(T)} \gg 1
$$

and when there is strong suppression of superconductivity in the vicinity of the sF and FS interfaces. The latter takes place if the parameter $\Gamma$

$$
\Gamma=\frac{\gamma \xi_{S}(T)}{\xi_{S}}, \xi_{S}(T)=\frac{\pi \xi_{S}}{2 \sqrt{1-T / T_{C}}}
$$

satisfies the conditions

$$
\Gamma p \gg 1, \Gamma q \gg 1 \text {. }
$$

Here

$$
\begin{aligned}
p^{-1} & =\frac{8}{\pi^{2}} R e \sum_{\omega=0}^{\infty} \frac{1}{\Omega^{2} \sqrt{\widetilde{\Omega}} \operatorname{coth} \frac{d_{F} \sqrt{\widetilde{\Omega}}}{2 \xi_{F}}}, \\
q^{-1} & =\frac{8}{\pi^{2}} R e \sum_{\omega=0}^{\infty} \frac{1}{\Omega^{2} \sqrt{\widetilde{\Omega}} \tanh \frac{d_{F} \sqrt{\widetilde{\Omega}}}{2 \xi_{F}}} .
\end{aligned}
$$

Note that in the limit $h=H / \pi T_{C} \gg 1$ and $d_{F} \gg \sqrt{2 / h} \xi_{F}$ the sums in (2.19) and (2.20) can be evaluated analytically resulting in

$$
\begin{gathered}
\beta=\frac{p-q}{p+q}=\sqrt{8} \sin \left(\frac{d_{F}}{\xi_{F}} \sqrt{\frac{h}{2}}+\frac{3 \pi}{4}\right) \exp \left(-\frac{d_{F}}{\xi_{F}} \sqrt{\frac{h}{2}}\right), \\
p+q=2 \sqrt{2 h}\left(T / T_{C}\right)^{2}, \quad p q=2 h\left(T / T_{C}\right)^{4} .
\end{gathered}
$$

In general, the phases of the order parameters in the s and $\mathrm{S}$ films are functions of the coordinate $x$. In the considered approximation the terms that take into account the coordinate dependence of the phases, are proportional to the small parameters $(\Gamma q)^{-1}$ and $(\Gamma p)^{-1}$ and therefore provide small corrections to the current. For this reason, in the first approximation we can assume that the phases in superconducting electrodes are constants independent of $x$. In the further analysis we denote the phases at the s-film by $\chi$ and at the right S-electrode by $\varphi$ (see Fig.2.1c).

The details of the calculations are summarized in the Appendix 4.7.1. These calculations show that the considered SIsFS junction has two modes of operation depending on the relation between the s layer thickness $d_{s}$ and the critical thickness $d_{s c}=(\pi / 2) \xi_{S}(T)$. For $d_{s}$ larger than $d_{s c}$, the s-film keeps its intrinsic superconducting properties (mode (1)), 
while for $d_{s} \leq d_{s c}$ superconductivity in the s-film exists only due to the proximity effect with the bulk S electrodes (mode (2)).

\subsubsection{Mode (1): SIs + sFS junction $d_{s} \geq d_{s c}$}

We begin our analysis with the regime when the intermediate s-layer is intrinsically superconducting. In this case it follows from the solution of the GL equations that the supercurrent flowing across the SIs, sF, and FS interfaces $\left(J\left(-d_{s}\right), J(0)\right.$ and $J\left(d_{F}\right)$, respectively) can be represented in the form (see the Appendix 4.7.1)

$$
\begin{gathered}
\frac{J_{S}\left(-d_{s}\right)}{J_{G}}=\frac{\delta_{s}\left(-d_{s}\right)}{\Gamma_{B I} \Delta_{0}} \sin (\chi), J_{G}=\frac{\pi \Delta_{0}^{2} \mathcal{A}_{B}}{4 e \rho_{S} T_{C} \xi_{S}(T)}, \\
\frac{J_{S}(0)}{J_{G}}=\frac{J_{S}\left(d_{F}\right)}{J_{G}}=\frac{\Gamma(p-q)}{2 \Delta_{0}^{2}} \delta_{s}(0) \delta_{S}\left(d_{F}\right) \sin (\varphi-\chi),
\end{gathered}
$$

where $\Delta_{0}=\sqrt{8 \pi^{2} T_{C}\left(T_{C}-T\right) / 7 \zeta(3)}$ is the bulk value of order parameter in the S electrodes, $\mathcal{A}_{B}$ is the cross-sectional area of the structure, and $\zeta(z)$ is the Riemann zeta function. Here

$$
\begin{gathered}
\delta_{s}(0)=\frac{2 b(p-q) \cos (\varphi-\chi)-2 a(p+q)}{\Gamma\left[(p+q)^{2}-(p-q)^{2} \cos ^{2}(\varphi-\chi)\right]}, \\
\delta_{S}\left(d_{F}\right)=\frac{2 b(p+q)-2 a(p-q) \cos (\varphi-\chi)}{\Gamma\left((p+q)^{2}-(p-q)^{2} \cos ^{2}(\varphi-\chi)\right)},
\end{gathered}
$$

are the order parameters at the sF and FS interfaces, respectively (see Fig. 2.1b) and

$$
a=-\delta_{s}\left(-d_{s}\right) \sqrt{1-\frac{\delta_{s}^{2}\left(-d_{s}\right)}{2 \Delta_{0}^{2}}}, b=\frac{\Delta_{0}}{\sqrt{2}},
$$

where $\delta_{s}\left(-d_{s}\right)$ is the solution of the transcendental equation

$$
K\left(\frac{\delta_{s}\left(-d_{s}\right)}{\Delta_{0} \eta}\right)=\frac{d_{s} \eta}{\sqrt{2} \xi_{s}(T)}, \eta=\sqrt{2-\frac{\delta_{s}^{2}\left(-d_{s}\right)}{\Delta_{0}^{2}}}
$$

Here, $K(z)$, is the complete elliptic integral of the first kind. Substitution of $\delta_{s}\left(-d_{s}\right)=0$ into Eq. (2.28) leads to the expression fo ther critical s layer thickness $d_{s c}=(\pi / 2) \xi_{S}(T)$, which was used above.

For the calculation of the CPR we need to exclude the phase $\chi$ of the intermediate s layer from the expressions for the currents (2.23) and (2.24). The value of this phase is determined from the condition that the currents flowing across the Is and sF interfaces should be equal to each other. 
For large thickness of the middle s-electrode $\left(d_{s} \gg d_{s c}\right)$ the magnitude of the order parameter $\delta_{s}\left(-d_{s}\right)$ is close to that of the bulk material $\Delta_{0}$ and we may put $a=-b$ in Eqs.(2.25) and (2.26)

$$
\delta_{S}\left(d_{F}\right)=\delta_{s}(0)=\frac{\sqrt{2} \Delta_{0}}{\Gamma((p+q)-(p-q) \cos (\varphi-\chi))},
$$

resulting in

$$
J_{S}(0)=J_{S}\left(d_{F}\right)=\frac{J_{G} \beta \sin (\varphi-\chi)}{\Gamma(1-\beta \cos (\varphi-\chi))}
$$

together with the equation to determine $\chi$

$$
\frac{\Gamma}{\Gamma_{B I}} \sin (\chi)=\frac{\beta \sin (\varphi-\chi)}{1-\beta \cos (\varphi-\chi)}, \beta=\frac{p-q}{p+q} .
$$

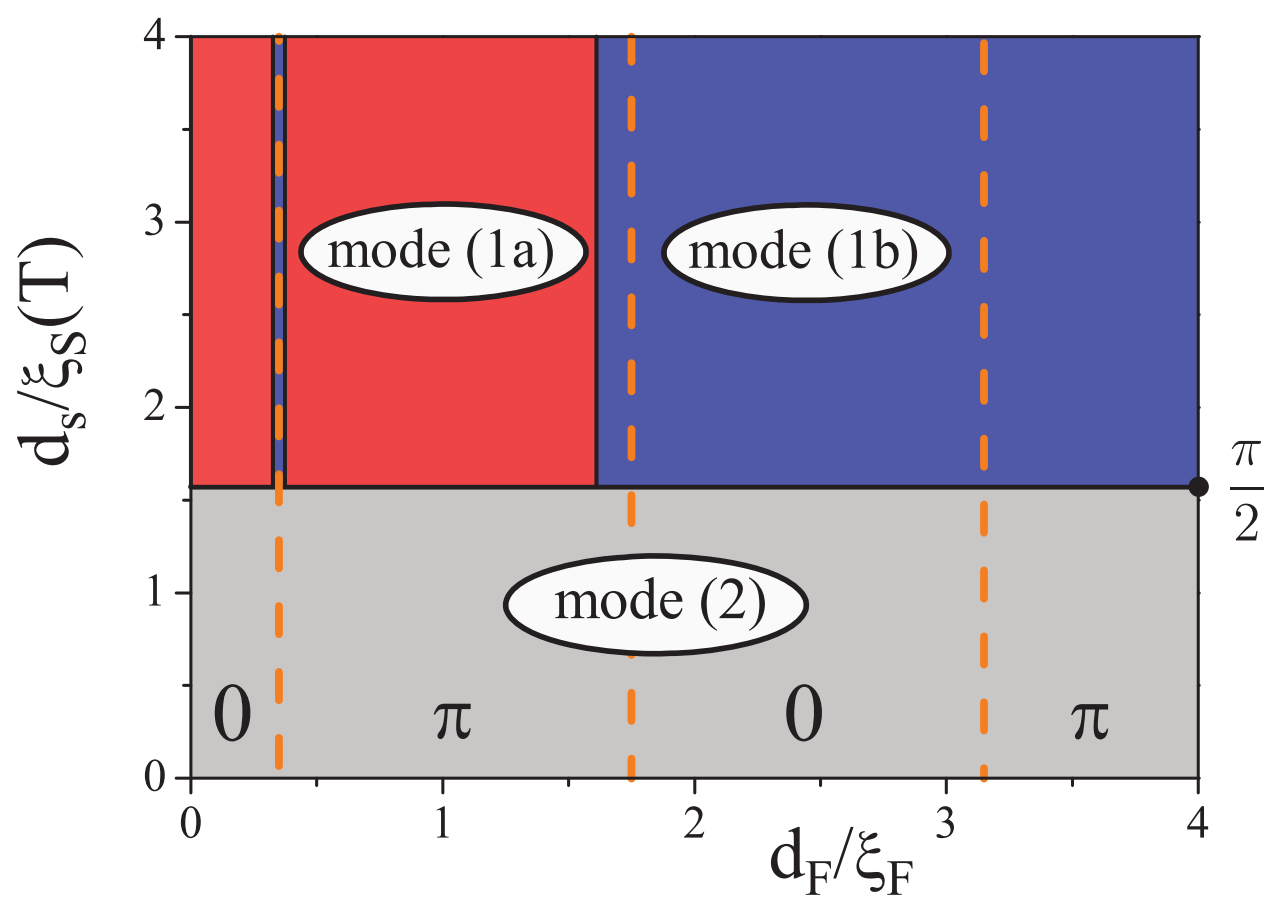

Figure 2.2. The phase diagram of the operation modes of the SIsFS structure in the $\left(d_{s}, d_{F}\right)$ plane. The bottom area corresponds to the mode (2) with fully suppressed superconductivity in the s-layer. The top part of the diagram, separated from the bottom one by the solid horizontal line, corresponds to the s-layer in the superconductive state. It provides the value of the s layer critical thickness $d_{s c}$. The upper left part indicates the mode (1a) with the weak place located at the SIs tunnel barrier. The upper right area as well as the thin valley around the first $0-\pi$ transition correspond to the mode (1b) with the weak place located at the sFS junction. The solid vertical lines provide the loci of the borders between the modes (1a) and (1b). The vertical dashed lines show the positions of $0-\pi$ transitions. The calculations have been done for $H=10 \pi T_{C}, \Gamma_{B I}=200$ and $\Gamma=5$ at $T=0.9 T_{C}$.

From (2.29), (2.30), and (2.31) it follows that in this mode the SIsFS structure can be considered as a pair of SIs and sFS junctions connected in series. Therefore, the 
properties of the structure are almost independent of the thickness $d_{s}$ and are determined by the junction with the smallest critical current.

Indeed, we can conclude from (2.31) that the phase $\chi$ of the s layer order parameter depends on the ratio of the critical current, $I_{C S I s} \propto \Gamma_{B I}^{-1}$, of its SIs part to that, $I_{C s F S} \propto$ $|\beta| \Gamma^{-1}$, of the sFS junction. The coefficient $\beta$ in (2.31) is a function of the $\mathrm{F}$ layer thickness, which becomes close to unity in the limit of small $d_{F}$ and exhibits damped oscillations with increase in $d_{F}$ (see the analytical expression for $\beta(2.21)$ ). That means that there is a range of thicknesses, $d_{F n}$, determined by the equation $\beta=0$, at which $J_{S} \equiv 0$ and there is a transition from the 0 to the $\pi$ state in the sFS part of the SIsFS junction. In other words, crossing the value $d_{F n}$ with an increase of $d_{F}$ provides a $\pi$ shift of $\chi$ relative to the phase of the $\mathrm{S}$ electrode.

In Fig.2.2 we clarify the classification of the operation modes and demonstrate the phase diagram in the $\left(d_{s}, d_{F}\right)$ plane, which follows from our analytical results (2.21)-(2.28). The calculations have been done at $T=0.9 T_{C}$ for $h=H / \pi T_{C}=10, \Gamma_{B I}=200$ and $\Gamma=5$. The structures with s-layer smaller than the critical thickness $d_{s c}=\pi \xi_{S}(T) / 2$ correspond to the mode (2) with fully suppressed superconductivity in the s layer. Conversely, the top part of the diagram corresponds to the s-layer in the superconductive state (mode (1)). This area is divided into two parts depending on whether the weak place is located at the tunnel barrier I (mode(1a)) or at the ferromagnetic F-layer (mode(1b)). The separating black solid vertical lines in the upper part of Fig.2.2 represent the locus of points where the critical currents of the SIs and sFS parts of the SIsFS junction are equal. The dashed lines give the locations of the points of 0 to $\pi$ transitions, $d_{F n}=\pi(n-3 / 4) \xi_{F} \sqrt{2 / h}, n=1,2,3 \ldots$, at which $J_{s}=0$. In the vicinity of these points there are the valleys of mode (1b) with the width, $\Delta d_{F n} \approx \xi_{F} \Gamma \Gamma_{B I}^{-1} h^{-1 / 2} \exp \{\pi(n-3 / 4)\}$, embedded into the areas occupied by mode (1a). For the set of parameters used for calculation of the phase diagram presented in Fig.2.2, there is only one valley with the width $\Delta d_{F 1} \approx \xi_{F} \Gamma \Gamma_{B I}^{-1} h^{-1 / 2} \exp \{\pi / 4\}$ located around the point $d_{F 1}=(\pi / 4) \xi_{F} \sqrt{2 / h}$ of the first 0 to $\pi$ transition.

\subsubsection{Mode (1a): Switchable $0-\pi$ SIs junction}

In the experimentally realized case [14-17] $\Gamma_{B I}^{-1} \ll|\beta| \Gamma^{-1}$ the condition is fulfilled and the weak place in the SIsFS structure is located at the SIs interface. In this approximation it follows from (2.31) that

$$
\chi \approx \varphi-\frac{2 q \Gamma}{(p-q) \Gamma_{B I}} \sin (\varphi)
$$




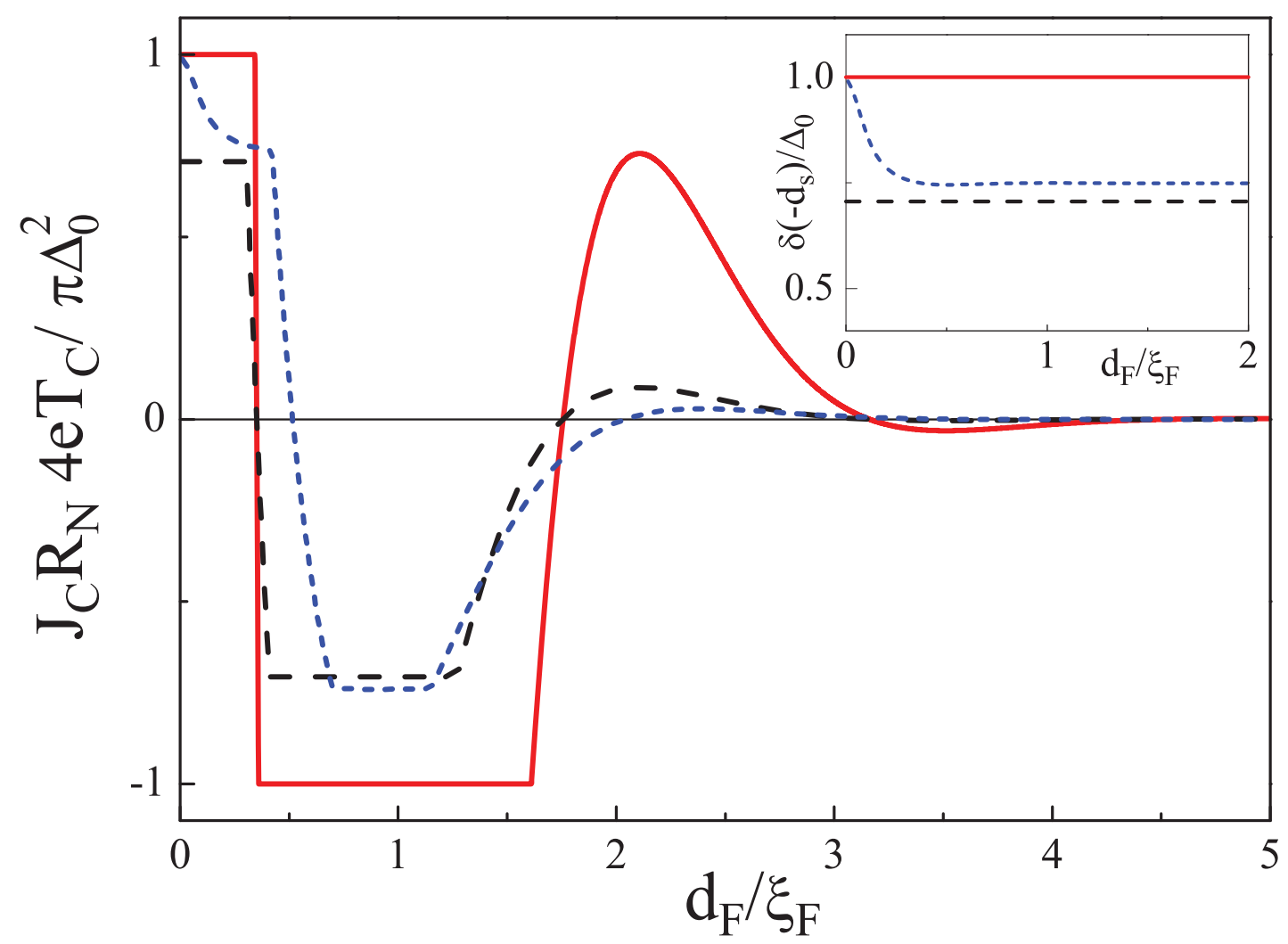

Figure 2.3. Critical current $J_{C}$ of the SIsFS structure versus F-layer thickness $d_{F}$ calculated at $T=0.9 T_{C}, H=10 \pi T_{C}, \Gamma_{B I}=200$ and $\Gamma=5$ for s layer thickness $d_{s}=2 \xi_{s}(T)$ slightly above the critical one $d_{s c}$. The inset shows the dependence of the pair potential $\delta_{s}\left(-d_{s}\right)$ at the Is interface of the s-layer versus the F-layer thickness $d_{F}$. The solid lines have been calculated for $d_{s} \gg d_{s c}$ from Eqs. (2.32) and (2.33). The dashed line is the result of calculations using the analytical expressions (2.23)-(2.28) for the thickness of the s-layer $d_{s}=2 \xi_{s}(T)$. The short-dashed line is the result of numerical calculations in the frame of the Usadel equations (2.2)-(2.11).

in the 0-state $\left(d_{F}<d_{F 1}\right)$ and

$$
\chi \approx \pi+\varphi-\frac{2 q \Gamma}{(p-q) \Gamma_{B I}} \sin (\varphi)
$$

in the $\pi$-state $\left(d_{F}>d_{F 1}\right)$. Substitution of these expressions into (2.30) results in

$$
J_{S}(\varphi)= \pm \frac{J_{G}}{\Gamma_{B I}}\left[\sin \varphi-\frac{\Gamma}{\Gamma_{B I}} \frac{1 \mp \beta}{2 \beta} \sin (2 \varphi)\right]
$$

for the 0 - and $\pi$ - states, respectively. It is seen that for $d_{F}<d_{F 1}$ the CPR (2.32) has the sinusoidal shape typical for SIS tunnel junctions with a small correction taking into account the suppression of superconductivity in the s layer due to proximity with the FS part of the complex sFS electrode. Its negative sign is typical for tunnel Josephson structures with composite NS or FS electrodes. [39,40]For $d_{F}>d_{F 1}$ the supercurrent changes its sign, thus 

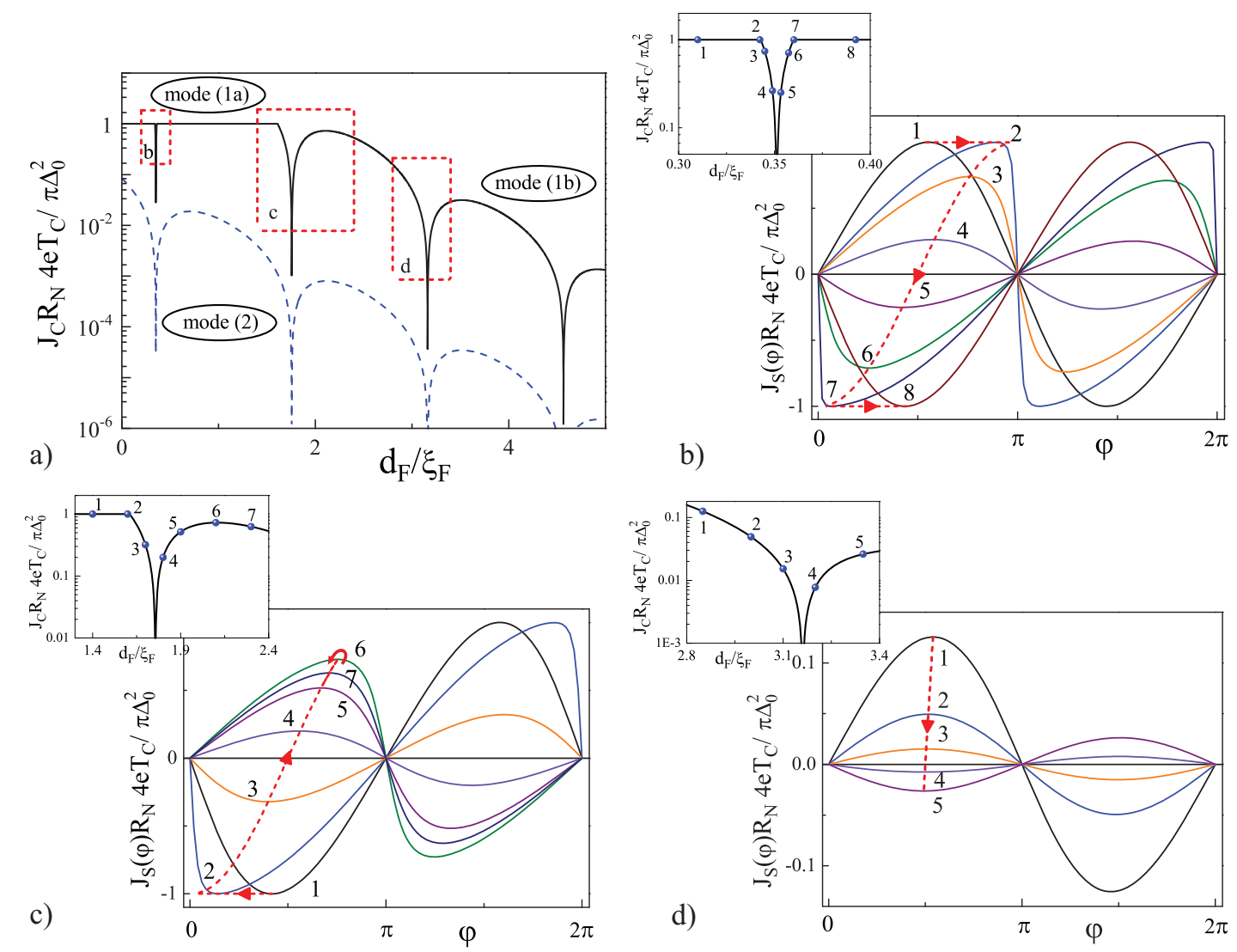

Figure 2.4. a) Magnitude of the critical current $J_{C}$ in the SIsFS structure versus F-layer thickness $d_{F}$ for two thickness of the middle s-layer, $d_{s}=5 \xi_{S}(T)>d_{s c}$ (solid line) and $d_{s}=0.5 \xi_{S}(T)<d_{s c}$ (dashed line) calculated at $T=0.9 T_{C}$ for $H=10 \pi T_{C}, \Gamma_{B I}=200$ and $\Gamma=5$. b)-d) CPR in the vicinity of $0-\pi$ transitions. The corresponding insets show the enlarged parts of $J_{C}\left(d_{F}\right)$ dependence enclosed in rectangles (a) and marked by the letters b-d, respectively. The digits on the insets show the points at which the $J_{S}(\varphi)$ curves have been calculated. The dashed lines (b)-(d) are the loci of critical points at which the $J_{S}(\varphi)$ dependence reaches its maximum value $J_{C}\left(d_{F}\right)$.

exhibiting the transition of the SIsFS junction into the $\pi$ state. It is important to note that in this mode the SIsFS structure may have almost the same value of the critical current in both 0 and $\pi$ states. It is unique property, which can not be realized in SFS devices studied before. For this reason we have identified this mode as "switchable $0-\pi$ SIS junction."

\subsubsection{Mode (1b): sFS junction}

Another limiting case is realized under the condition $\Gamma_{B I}^{-1} \gg|\beta| \Gamma^{-1}$. It is satisfied in the vicinity of the points of $0-$ to $\pi$ - transitions, $d_{F n}$, and for large $d_{F}$ values and high exchange fields $H$. In this mode (see Fig. 2.2) the weak place shifts to the sFS part of the SIsFS device and the structure transforms into a conventional SFS-junction with a complex SIs electrode. 
In the first approximation on $\Gamma /\left(\beta \Gamma_{B I}\right) \gg 1$ it follows from (2.30) and (2.31) that

$$
\chi=\frac{\Gamma_{B I}}{\Gamma} \frac{\beta \sin (\varphi)}{1-\beta \cos (\varphi)},
$$

resulting in

$$
J_{S}(\varphi)=\frac{J_{G} \beta}{\Gamma(1-\beta \cos \varphi)}\left(\sin \varphi-\frac{\Gamma_{B I}}{2 \Gamma} \frac{\beta \sin (2 \varphi)}{(1-\beta \cos \varphi)}\right) .
$$

The shape of the CPR for $\chi \rightarrow 0$ coincides with that previously found in SNS and SFS Josephson devices [37]. It transforms to the sinusoidal form for sufficiently large thickness of the $\mathrm{F}$ layer. For small thickness of the F-layer as well as in the vicinity of $0-\pi$ transitions, significant deviations from the sinusoidal form may occur.

The transition between modes (1a) and (1b) is also demonstrated in Fig. 2.3. It shows the dependence of the critical current $J_{C}$ across the SIsFS structure versus the Flayer thickness $d_{F}$. The inset in Fig.2.3 demonstrates the magnitude of the order parameter at the Is interface as a function of $d_{F}$. The solid lines in Fig. 2.3 give the shape of $J_{C}\left(d_{F}\right)$ and $\delta_{0}\left(-d_{s}\right)$ calculated from (2.32)-(2.33). These equations are valid in the limit $d_{s} \gg d_{s c}$ and do not take into account possible suppression of superconductivity in the vicinity of a tunnel barrier due to proximity with the FS part of the device. The dashed lines are the result of calculations using the analytical expressions (2.23)-(2.28) for the thickness of the s-layer $d_{s}=2 \xi_{s}(T)$, which slightly exceeds the critical one, $d_{s c}=(\pi / 2) \xi_{s}(T)$. These analytical dependencies are calculated at $T=0.9 T_{C}$ for $H=10 \pi T_{C}, \Gamma_{B I}=200, \Gamma=5$, and $\gamma_{B}=0$. The short-dashed curves are the results of numerical calculations performed selfconsistently in the frame of the Usadel equations (2)-(11) for the corresponding set of the parameters $T=0.9 T_{C}$ for $H=10 \pi T_{C}, \gamma_{B I}=1000, \gamma=1, \gamma_{B}=0.3$ and the same thickness of the s layer $d_{s c}=(\pi / 2) \xi_{s}(T)$. The interface parameters $\gamma_{B I}=1000$ and $\gamma=1$ are chosen the same as for the analytical case. The choice of $\gamma_{B}=0.3$ allows one to take into account the influence of mismatch which generally occurs at the sF and FS boundaries.

It can be seen that there is a qualitative agreement between the shapes of the three curves. For small $d_{F}$ the structure is in the 0 -state mode (1a) regime. The difference between dashed and short dashed lines in this area is due to the fact that the inequalities (2.18) are not fulfilled for very small $d_{F}$. The solid and short dashed curves start from the same value since for $d_{F}=0$ the sFS electrode becomes a single spatially homogeneous superconductor. For $d_{s}=2 \xi_{s}(T)$ the intrinsic superconductivity in the $\mathrm{s}$ layer is weak and is partially suppressed with $d_{F}$ increase (see the inset in Fig. 2.3). This suppression is accompanied by 
a rapid drop of the critical current. It can be seen that starting from the value $d_{F} \approx 0.4 \xi_{F}$ our analytical formulas (2.23)-(2.28) are accurate enough. The larger is $d_{s}$, the better is the agreement between numerical and analytical results due to the better applicability of the GL equations in the s layer. With further $d_{F}$ increase the structure passes through a valley of the mode (1b) state, located in the vicinity of the 0 to $\pi$ transition, and comes into the $\pi$-state of mode (1a). Finally for $d_{F} \gtrsim 1.6 \xi_{F}$ there is a transition from mode (1a) to mode (1b), which is accompanied by damped oscillation of $J_{C}\left(d_{F}\right)$ with increase in $d_{F}$.

\subsubsection{Mode (2): SInFS junction $d_{s} \leq d_{s c}$}

For $d_{s} \leq d_{s c}$ intrinsic superconductivity in the $s$ layer is completely suppressed resulting in formation of the complex -InF- weak-link area, where ' $n$ ' marks the intermediate $\mathrm{s}$ film in the normal state. In this parameter range the weak link is always located in the tunnel barrier and the CPR has sinusoidal shape,

$$
J_{S}(\varphi)=\frac{J_{G}}{\sqrt{2}} \frac{(p-q) \sin \varphi}{2 p q \Gamma \Gamma_{B I} \cos \frac{d_{s}}{\xi_{s}(T)}+\left[2 p q \Gamma+(p+q) \Gamma_{B I}\right] \sin \frac{d_{s}}{\xi_{s}(T)}} .
$$

In the vicinity of the critical thickness $d_{s} \lesssim d_{s c}$, the factor $\cos \left(d_{s} / \xi_{S}(T)\right)$ in $(2.34)$ is small and the supercurrent is given by the expression

$$
J_{S}(\varphi)=\frac{J_{G}}{2 \sqrt{2}} \frac{(p-q) \sin \varphi}{2 p q \Gamma+(p+q) \Gamma_{B I}}
$$

Further decrease of $d_{s}$ to the limit $d_{s} \ll d_{s c}$ leads to

$$
J_{S}(\varphi)=\frac{J_{G}}{\sqrt{2}} \frac{(p-q) \sin \varphi}{2 p q \Gamma \Gamma_{B I}}
$$

The magnitude of the critical current in (2.36) is close to that in the well-known case of SIFS junctions in the appropriate regime.

\subsubsection{Current-Phase Relation}

In the previous section we have demonstrated that variation in the thickness of the ferromagnetic layer should lead to the transformation of the CPR of the SIsFS structure. Figure 2.4a illustrates the $J_{C}\left(d_{F}\right)$ dependencies calculated from expressions (2.23)-(2.28) at $T=0.9 T_{C}$ for $H=10 \pi T_{C}, \gamma_{B}=0, \Gamma_{B I} \approx 200$ and $\Gamma \approx 5$ for two thickness of the s layer $d_{s}=5 \xi_{S}(T)$ (solid line) and $d_{s}=0.5 \xi_{S}(T)$ (dashed line). In Figs.2.4b-d we enlarge the parts of the $J_{C}\left(d_{F}\right)$ dependence enclosed in rectangles labeled by the letters b, c and d in 
Fig.2.4a and mark by digits the points where the $J_{S}(\varphi)$ curves have been calculated. These curves are marked by the same digits as the points in the enlarged parts of the $J_{C}\left(d_{F}\right)$ dependencies. The dashed lines in the Figs.2.4b-d are the loci of critical points at which the $J_{S}(\varphi)$ dependence reaches its maximum value, $J_{C}\left(d_{F}\right)$.

Figure $2.4 \mathrm{~b}$ presents the mode (1b) valley, which divides the mode (1a) domain into 0 - and $\pi$ - states regions. In the mode (1a) domain the SIsFS structure behaves as SIs and sFS junctions connected in series. Its critical current equals the minimal one among the critical currents of the SIs $\left(J_{C S I s}\right)$ and sFS $\left(J_{C s F S}\right)$ parts of the device. In the considered case the thickness of the $\mathrm{s}$ film is sufficiently large to prevent suppression of superconductivity. Therefore, $J_{C S I s}$ does not change when moving from point 1 to point 2 along $J_{C} d_{F}$ dependence. At point 2 , when $J_{C S I s}=J_{C s F S}$, we arrive at the border between modes (1a) and (1b). It is seen that at this point there is maximum deviation of $J_{S}(\varphi)$ from the sinusoidal shape. Further increase of $d_{F}$ leads to the $0-\pi$ transition, when the parameter $\beta$ in (2.33) becomes small and $J_{S}(\varphi)$ is almost restored to its sinusoidal shape. Beyond the area of the 0 to $\pi$ transition, the critical current changes its sign and the CPR starts to deform again. The deformation achieves its maximum at the point 7 located at the other border between modes (1a) and (1b). The displacement from point 7 to point 8 along the $J_{C}\left(d_{F}\right)$ dependence leads to recovery of a sinusoidal CPR.

Figure 2.4c presents the transition from the $\pi$-state of mode (1a) to mode (1b) with $d_{F}$ increase. It is seen that the offset from point 1 to points $2-5$ along $J_{C}\left(d_{F}\right)$ results in a transformation of the CPR similar to that shown in Fig.2.4b during displacement in the direction from point 1 to points 2-6. The only difference is the starting negative sign of the critical current. However, this behavior of the CPRalong with the close transition between modes lead to formation of a well-pronounced kink in the $J_{C}\left(d_{F}\right)$ dependence. Furthermore, in contrast to Fig. 2.4b at point 6, the junction is still in mode (1b) and remains in this mode with further increase in $d_{F}$. At point 6 the critical current achieves its maximum value and it decreases along the dashed line for larger $d_{F}$.

Figure 2.4d shows the transformation of the CPR in the vicinity of the next 0 to $\pi$ transition in mode (1b). There is a small deviation from the sinusoidal shape at point 1 , which vanishes exponentially with an increase of $d_{F}$.

In mode (2) (the dashed curve in Fig.2.4a) intrinsic superconductivity in the s layer is completely suppressed resulting in the formation of a complex -InF- weak link region and the CPR becomes sinusoidal (2.34). 

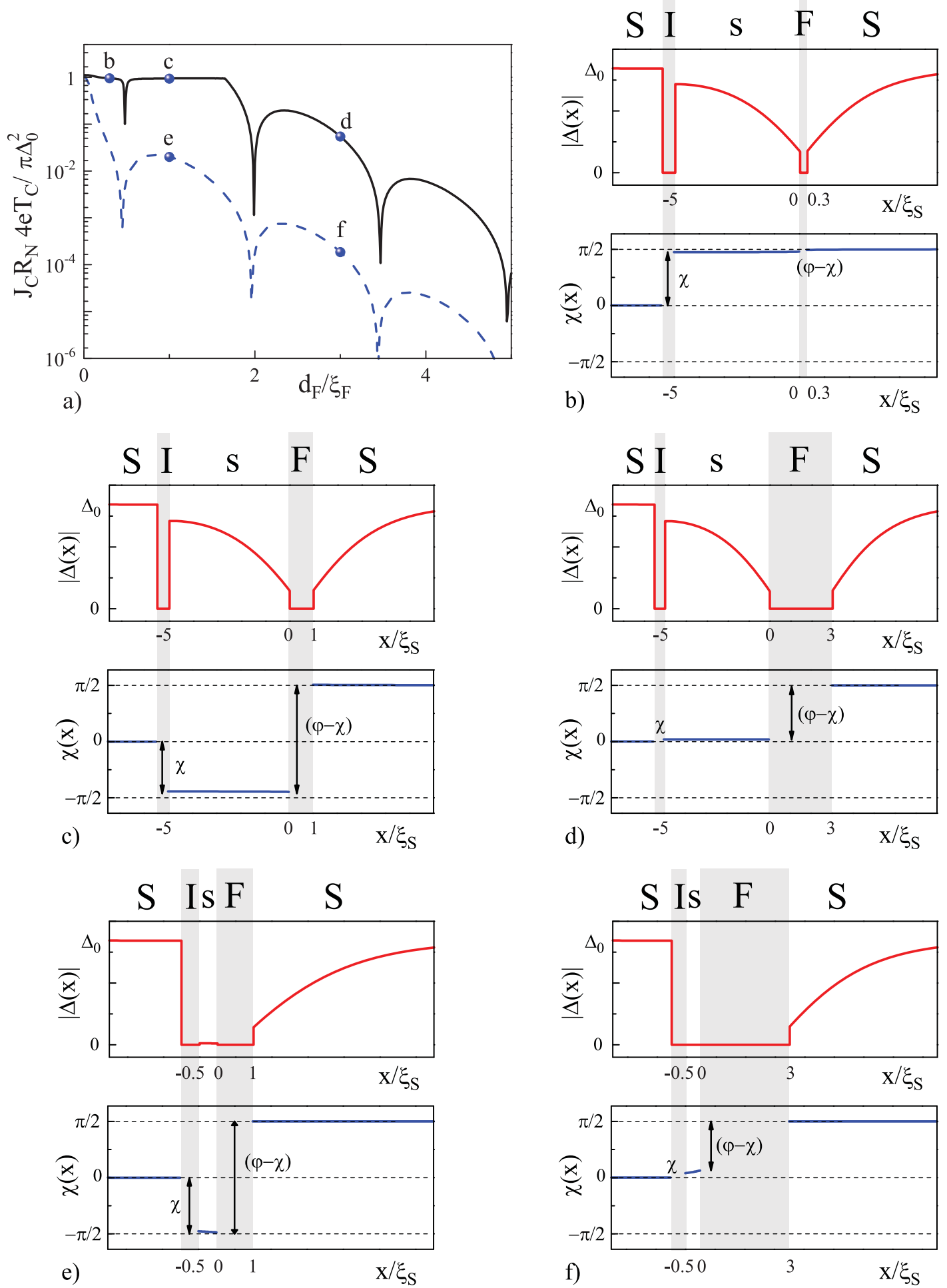

Figure 2.5. a) Magnitude of critical current $J_{C}$ of the SIsFS structure versus F-layer thickness $d_{F}$ calculated at $T=0.5 T_{C}$ for $H=10 \pi T_{C}, \gamma_{B I}=1000, \gamma=1$ and two thicknesses of the s film $d_{s}=5 \xi_{S}$ (solid line) and $d_{s}=0.5 \xi_{S}$ (dashed line). The letters on $J_{C}\left(d_{F}\right)$ give the points at which the coordinate dependencies of the magnitude of the order parameter, $|\Delta(x)|$, and phase difference across the structure, $\chi$, have been calculated. These curves are shown in the panels b)-f) as the upper and lower panels, respectively. 


\subsection{Arbitrary temperature}

At arbitrary temperatures the boundary problem (2.2)-(2.11) goes beyond the assumptions of GL formalism and requires a self-consistent solution. We have performed it numerically in terms of the nonlinear Usadel equations in an iterative manner. All calculations were performed for $T=0.5 T_{C}, \xi_{S}=\xi_{F}, \gamma_{B I}=1000, \gamma_{B F S}=0.3$ and $\gamma=1$.

Calculations show that at the selected transparency of the tunnel barrier $\left(\gamma_{B I}=\right.$ 1000) the suppression of superconductivity in the left electrode is negligibly small. This allows one to select the thickness of the left $\mathrm{S}$ electrode $d_{S L}=2 \xi_{S}$ without any loss of generality. On the contrary, proximity of the right $\mathrm{S}$ electrode to the $\mathrm{F}$ layer results in strong suppression of superconductivity at the FS interface. Therefore the pair potential of the right $\mathrm{S}$ electrode reaches its bulk value only at thickness $d_{S R} \gtrsim 10 \xi_{S}$. It is for these reasons we have chosen $d_{S R}=10 \xi_{S}$ for the calculations.

Furthermore, the presence of a low-transparency tunnel barrier in the considered SIsFS structures limits the magnitude of the critical current $J_{C}$ by a value much smaller than the depairing current of the superconducting electrodes. This allows one to neglect nonlinear corrections to coordinate dependence of the phase in the $\mathrm{S}$ banks.

The results of the calculations are summarized in Fig. 2.5. Figure 2.5a shows the dependence of $J_{C}$ of the SIsFS structure on the F-layer thickness $d_{F}$ for relatively large $d_{s}=5 \xi_{S}$ (solid) and small $d_{s}=0.5 \xi_{S}$ (dashed) s-film thickness. The letters on the curves indicate the points at which the coordinate dependencies of the magnitude of the order parameter, $|\Delta(x)|$, and phase difference across the structure, $\chi$, have been calculated for the phase difference $\varphi=\pi / 2$. These curves are shown in the panels b)-f) of the Fig.2.5 as the upper and lower plots, respectively. There is direct correspondence between the letters, b, c, d, e, f, on the $J_{C}\left(d_{F}\right)$ curves and the labels, b), c), d), e), f), of the panels.

It is seen that the qualitative behavior of the $J_{C}\left(d_{F}\right)$ dependence at $T=0.5 T_{C}$ remains similar to that obtained in the frame of the GL equations for $T=0.9 T_{C}$ (see Fig.2.4a). Furthermore, the modes of operation discussed above remain relevant too. The panels b)-f) in Fig. 2.5 make this statement more clear.

At the point marked by the letter 'b', the s-film is sufficiently thick, $d_{s}=5 \xi_{S}$, while the $\mathrm{F}$ film is rather thin, $d_{F}=0.3 \xi_{F}$, and therefore the structure is in the 0 - state of mode (1a). In this regime the phase mainly drops across the tunnel barrier, while the phase shifts at the s-film and in the $\mathrm{S}$ electrodes are negligibly small(see the bottom plot in Fig.2.5b). 
At the point marked by the letter 'c' $\left(d_{s}=5 \xi_{S}, d_{F}=\xi_{F}\right)$, the structure is in the $\pi$ state of mode (1a). It is seen from Fig.2.5c that there is a phase jump at the tunnel barrier and an additional $\pi$-shift occurs between the phases of the $\mathrm{S}$ and $\mathrm{s}$ layers.

For $d_{F}=3 \xi_{F}$ (Fig.2.5d) the position of the weak place shifts from the SIs to the sFS part of the SIsFS junction. Then the structure starts to operate in the mode (1b). It is seen that the phase drop across the SIs part is small, while it is $\varphi-\chi \approx \pi / 2$ across the F layer, as it should be in SFS junctions with SIs and S electrodes.

At the points marked by the letters 'e' and 'f', thickness of the s-layer $d_{s}=0.5 \xi_{S}$ is less than its critical value. Then superconductivity in the s-spacer is suppressed due to the proximity with the F film and SIsFS device operates in the mode (2). At $d_{F}=\xi_{F}$ (the dot 'e' in Fig.2.5a and the panel Fig.2.5e) the position of the weak place is located in the SIs part of the structure and there is an additional $\pi$-shift of phase across the F film. As a result, the SIsFS structure behaves like an SInFS tunnel $\pi$-junction. The unsuppressed residual value of the pair potential is due to the proximity with the right S-electrode and it disappears with the growth of the F-layer thickness, which weakens this proximity effect. At $d_{F}=3 \xi_{F}$ (Fig.2.5f) the weak place is located in the $\mathrm{F}$ part of IsF trilayer. Despite strong suppression of the pair potential in the s-layer, the distribution of the phase inside the IsF weak place has a rather complex structure, which depends on the thicknesses of the $\mathrm{s}$ and $\mathrm{F}$ layers.

\subsubsection{Temperature crossover from 0 to $\pi$ states}

The temperature-induced crossover from 0 to $\pi$ states in SFS junctions was described in [26] in structures with sinusoidal CPR. It was found that the transition takes place in a relatively broad temperature range.

Our analysis of SIsFS structure (see Fig.2.6a) shows that smoothness of 0 to $\pi$ transition strongly depends on the CPR shape. This phenomenon was not analyzed before since almost all previous theoretical results were obtained within a linear approximation leading in a sinusoidal CPR. To prove the dependence on the CPR shape, we have calculated numerically the set of $J_{C}(T)$ curves for a number of $\mathrm{F}$ layer film thicknesses $d_{F}$. We have chosen the thickness of the intermediate superconductor $d_{S}=5 \xi_{S}$ in order to have SIsFS device in mode(1a) and we have examined the parameter range $0.3 \xi_{F} \leq d_{F} \leq \xi_{F}$, in which the structure exhibits the first 0 to $\pi$ transition. The borders of the $d_{F}$ range are chosen in such a way that the SIsFS contact is in either the $0-\left(d_{F}=0.3 \xi_{F}\right)$ or the $\pi$ - $\left(d_{F}=\xi_{F}\right)$ state 

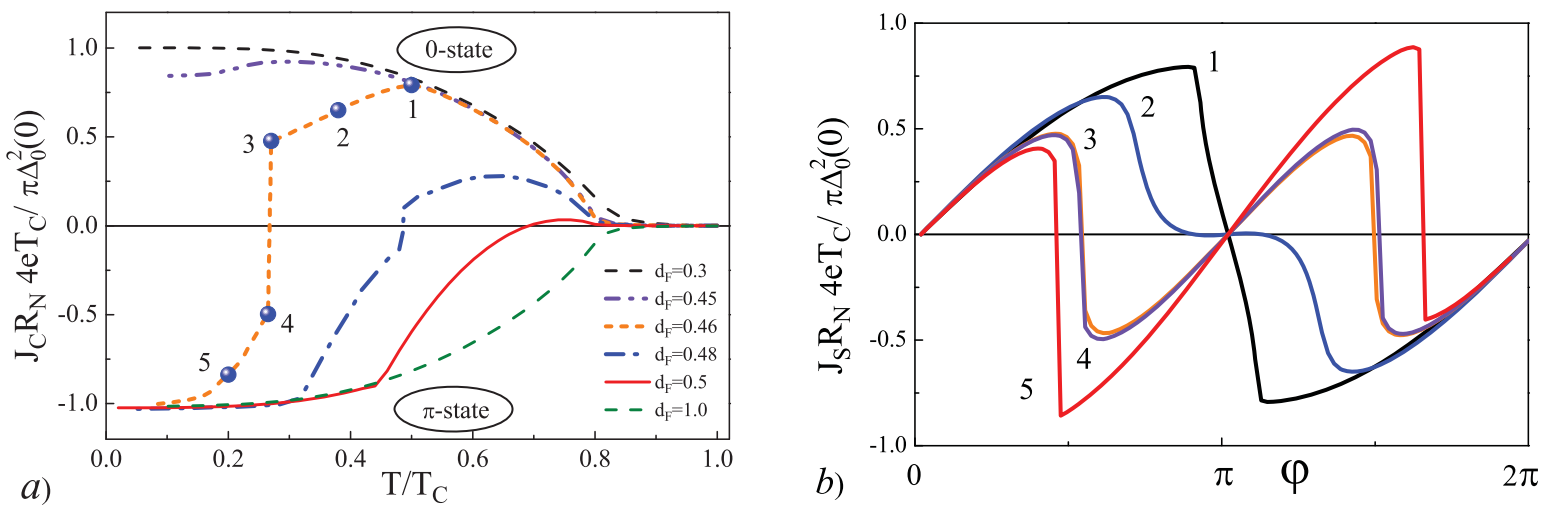

Figure 2.6. a) Critical current $J_{C}$ of the SIsFS structure versus temperature $T$ for various Flayer thicknesses $d_{F}$ in the vicinity of the 0 to $\pi$ transition. The dashed envelopes show the temperature dependence in the 0- (top) and $\pi$ - (bottom) states. b) CPR of the structure with $d_{F}=0.46 \xi_{F}$ for a set of temperatures in the vicinity of the $0-\pi$ transition. Each curve corresponds to the point marked in (a). Note that the curves (3) and (4) almost coincide but correspond to different ground states of the junction, 0 - and $\pi$, respectively. The calculations have been performed numerically for $d_{s}=5 \xi_{S}, H=10 \pi T_{C}, \gamma_{B I}=1000$, $\gamma_{B}=0.3$, and $\gamma=1$.

in the whole temperature range. The corresponding $J_{C}(T)$ dependencies (dashed lines in Fig. 2.6a) provide the envelope of a set of $J_{C}(T)$ curves calculated for the considered range of $d_{F}$. It is clearly seen that in the vicinity of $T_{C}$ the decrease of $d_{F}$ results in creation of the temperature range where the 0 -state exists. The point of 0 to $\pi$ transition shifts to lower temperatures with decreasing $d_{F}$. For $d_{F} \gtrsim 0.5 \xi_{F}$ the transition is rather smooth since for $T \geq 0.8 T_{C}$ the junction stays in the mode (2) (with suppressed superconductivity) and deviations of the CPR from $\sin (\varphi)$ are small. Thus the behavior of the $J_{C}(T)$ dependencies in this case can be easily described by the analytical results from Sec.2.3.3.

The situation drastically changes at $d_{F}=0.46 \xi_{F}$ (short-dashed line in Fig.2.6a). For this thickness the point of the 0 to $\pi$ transition shifts to $T \approx 0.25 T_{C}$. This shift is accompanied by an increase of the amplitudes of the higher harmonics of the CPR (see Fig.2.6b). As a result, the shape of the CPR is strongly modified, so that in the interval $0 \leq \varphi \leq \pi$ the CPR curves are characterized by two values, $J_{C 1}$ and $J_{C 2}$, as is known from the case of SFcFS constrictions [41]. In general, $J_{C 1}$ and $J_{C 2}$ differ both in sign and in magnitude and $J_{C}=\max \left(\left|J_{C 1}\right|,\left|J_{C 2}\right|\right)$. For $T>0.25 T_{C}$ the junction is in the 0 -state and $J_{C}$ grows with decrease of $T$ up to $T \approx 0.5 T_{C}$. Further decrease of $T$ is accompanied by suppression of the critical current. In the vicinity of $T \approx 0.25 T_{C}$ the difference between $\left|J_{C 1}\right|$ and $\left|J_{C 2}\right|$ becomes negligible and the system starts to develop an instability that eventually shows up as a sharp jump from the 0 to the $\pi$ state. After the jump, $\left|J_{C}\right|$ continuously 


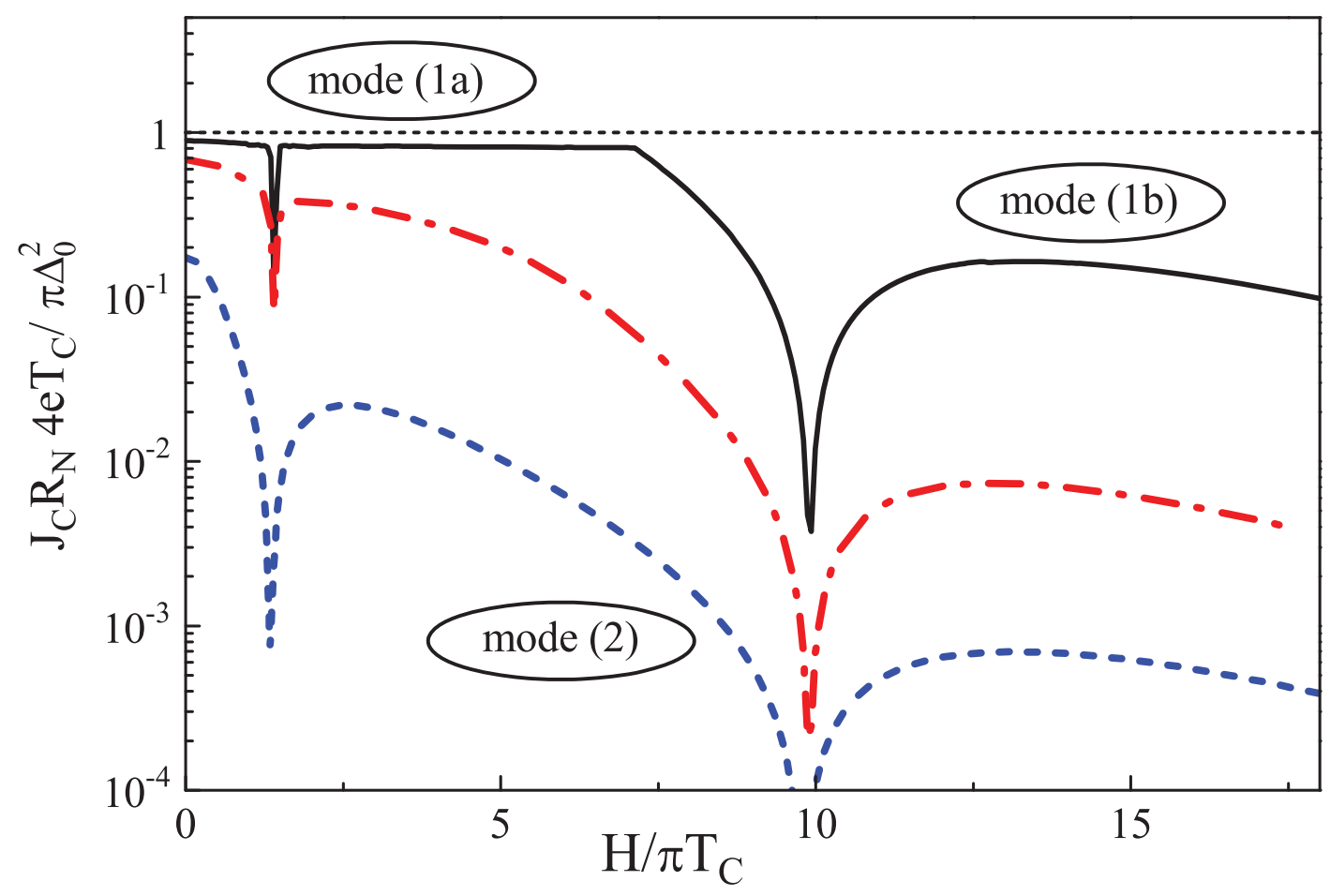

Figure 2.7. The magnitude of critical current $J_{C}$ of the SIsFS structure versus exchange field $H$ for thick $d_{s}=5 \xi_{S}$ (solid), thin $d_{s}=0.5 \xi_{S}$ (dashed) and intermediate $d_{s}=3 \xi_{S}$ (dash-dotted) s-layer thickness. The plot demonstrates the possibility of $0-\pi$ transition by varying the effective exchange field. The calculations have been performed for $T=0.5 T_{C}$, $d_{F}=2 \xi_{F}, \gamma_{B I}=1000, \gamma_{B}=0.3, \gamma=1$.

increases as $T$ goes to zero.

It is important to note that this behavior should always be observed in the vicinity of a $0-\pi$ transition, i.e., in the range of parameters in which the amplitude of the first harmonic is small compared to higher harmonics. However, the closer the temperature is to $T_{C}$, the less pronounced are the higher CPR harmonics and the smaller is the magnitude of the jump. This fact is illustrated by the dash-dotted line showing $J_{C}(T)$ calculated for $d_{F}=0.48 \xi_{F}$. A jump in the curves calculated for $d_{F} \geq 0.5 \xi_{F}$ also exists, but it is small and can not be resolved on the scale used in Fig. 2.6a.

At $d_{F}=0.45 \xi_{F}$ (dash-dot-dotted line in Fig. 2.6) the junction is always in the 0state and there is only small suppression of the critical current at low temperatures despite the realization of a non-sinusoidal CPR.

Thus the calculations clearly show that it is possible to realize a set of parameters of SIsFS junctions where thermally induced $0-\pi$ crossover can be observed and controlled by temperature variation. 


\subsubsection{0 to $\pi$ crossover by changing the effective exchange energy in external magnetic field}

The exchange field is an intrinsic microscopic parameter of a ferromagnetic material which cannot be controlled directly by application of an external field. However, spin splitting in F-layers can be provided by both an internal exchange field and an external magnetic field [42,43], resulting in generation of an effective exchange field, which is equal to their sum. The practical realization of this effect is a challenge since it is difficult to fulfill the special requirements $[42,43]$ on the thickness of the $\mathrm{S}$ electrodes and the SFS junction geometry.

Another opportunity can be realized in soft diluted ferromagnetic alloys like $\mathrm{Fe}_{0.01} \mathrm{Pd}_{0.99}$. Investigations of the magnetic properties [18] of these materials have shown that below $14 \mathrm{~K}$ they exhibit ferromagnetic order due to the formation of weakly coupled ferromagnetic nanoclusters. In the clusters, the effective spin polarization of the Fe ions is about $4 \mu_{B}$, corresponding to that in the bulk $\mathrm{Pd}_{3} \mathrm{Fe}$ alloy. It was demonstrated that the hysteresis loops of $\mathrm{Fe}_{0.01} \mathrm{Pd}_{0.99}$ films have the form typical of nanostructured ferromagnets with weakly coupled grains (the absence of domains; a small coercive force; a small interval of magnetization reversal, where the magnetization changes its direction following changes in the applied magnetic field; and a prolonged part, where the component of the magnetization vector along the applied field grows gradually).

The small of concentration of the $\mathrm{Pd}_{3}$ Fe clusters and their ability to follow variations in the applied magnetic field may result in generation of $H_{\text {eff }}$, which is of the order of

$$
H_{e f f} \approx H \frac{n_{\uparrow} V_{\uparrow}-n_{\downarrow} V_{\downarrow}}{n V}
$$

Here $n$ is the concentration of electrons within a physically small volume $V$, in which one performs an averaging of the Greens functions in the transformation to a quasiclassical description of superconductivity, $n_{\uparrow, \downarrow}$ and $V_{\uparrow, \downarrow}$ are the values describing the spin polarized parts of $n$ and parts of the volume $V$, which they occupy, respectively. A similar kind of $H_{\text {eff }}$ nucleates in NF or SF proximity structures, which are composed from thin layers [45-48]. There is an interval of applied magnetic fields $H_{\text {ext }}$ where the alloy magnetization changes its direction and the concentrations $n_{\uparrow, \downarrow}$ depend on the pre-history of application of the field $[12,15]$, providing the possibility to control $H_{\text {eff }}$ by an external magnetic field.

Derivation of the possible relationships between $H_{e f f}$ and $H_{e x t}$ is outside the scope of 
this paper. Below we will concentrate only on an assessment of the intervals in which $H_{e f f}$ should be changed to ensure the transition of a SIsFS device from the 0 to the $\pi$ state. To do this, we calculate the $J_{C}(H)$ dependencies presented in Fig. 2.7. The calculations have been done for a set of structures with $d_{F}=2 \xi_{F}$ and s-films thickness ranging from thick, $d_{S}=5 \xi_{S}$ (solid line) to an intermediate value $d_{S}=2 \xi_{S}$ (dashed-dotted line) and finishing with a thin film having $d_{S}=0.5 \xi_{S}$ (dashed line). It is clearly seen that these curves have the same shape as the $J_{C}\left(d_{F}\right)$ dependencies presented in Sec.2.3. For $d_{S}=5 \xi_{S}$ and $H \lesssim 7 \pi T_{C}$ the magnitude of $J_{C}$ is almost independent of $H$, but it changes sign at $H \approx 1.25 \pi T_{C}$ due to the $0-\pi$ transition. It is seen that for the transition, while maintaining the normalized current value at a level close to unity, changes of $H$ are required approximately of the order of $0.1 \pi T_{C}$ or $10 \%$. For $d_{S}=2 \xi_{S}$ and $H \lesssim 3 \pi T_{C}$, it is necessary to change $H$ by $20 \%$ to realize such a transition. In this case the value of the normalized current is at the level 0.4 . In mode 2 the transition requires a $100 \%$ change of $H$, which is not practical.

\subsection{Discussion}

We have performed a theoretical study of magnetic SIsFS Josephson junctions. At $T \leq T_{C}$ calculations have been performed analytically in the frame of the GL equations. For arbitrary temperatures we have developed a numerical code for self-consistent solution of the Usadel equations. We have outlined several modes of operation of these junctions. For the s-layer in the superconducting state they are S-I-sfS or SIs-F-S devices with the weak place located at the insulator (mode (1a)) or at the F-layer (mode (1b)), respectively. For a small s-layer thickness, its intrinsic superconductivity is completely suppressed, resulting in formation of an InF weak place (mode (2)). We have examined the shape of $J_{S}(\varphi)$ and the spatial distribution of the modulus of the pair potential and its phase difference across the SIsFS structure in these modes.

For mode (1) the shape of the CPR can substantially differ from sinusoidal even in the vicinity of $T_{C}$. The deviations are largest when the structure is close to the crossover between the modes (1a) and (1b). This effect results in kinks in the dependencies of $J_{C}$ on temperature and on parameters of the structure (thickness of the layers $d_{F}, d_{s}$ and exchange energy $H$ ) as illustrated in Fig.2.4 on $J_{C}\left(d_{F}\right)$ curves. The transformation of the CPR is even more important at low temperatures. For $T \lesssim 0.25 T_{C}$ a sharp $0-\pi$ transition can be realized induced by a small temperature variation (Fig. 2.6). This instability must be taken 
into account when using the structures as memory elements. On the other hand, this effect can be used in detectors of electromagnetic radiation, where absorption of a photon in the F layer will provide local heating leading to development of an instability and subsequent phonon registration.

We have shown that suppression of the order parameter in a thin s-film due to the proximity effect leads to decrease of the product $J_{C} R_{N}$ in both $0-$ and $\pi$-states. On the other hand, the proximity effect may also support s-layer superconductivity due to the impact of S electrodes. In mode (1a) theproduct $J_{C} R_{N}$ in the 0 - and $\pi$-states can achieve values typical for SIS tunnel junctions.

In mode (2) a sinusoidal CPR is realized. Despite that, the distribution of the phase difference $\chi(x)$ in the IsF weak place may have a complex structure, which depends on the thickness of the s and F layers. These effects should influence the dynamics of a junction in its $a c$-state and deserve further study.

Further, we have also shown that in mode (1a) nearly $10 \%$ change in the exchange energy can cause a $0-\pi$ transition, i.e. changing the sign of the $J_{C} R_{N}$ product, while maintaining its absolute value. This unique feature can be implemented in mode (1a), since in it changes of the exchange energy determine only the presence or absence of a $\pi$ shift between s and S electrodes and do not affect the magnitude of the critical current of the SIs part of the SIsFS junction.

In mode (1b), the $\mathrm{F}$ layer becomes a part of the weak-link area. In this case the $\pi$ shift initiated by the change in $H$ must be accompanied by changes of the magnitude of $J_{C}$ due to the oscillatory nature of superconducting correlations in the F film. The latter may lead to a very complex and irregular dependence $J_{C}\left(H_{\text {ext }}\right)$, such as has been observed in NbPdFe-Nb SFS junctions(see Fig.3 in Ref. [14]). In contrast, to that the $J_{C}\left(H_{e x t}\right)$ curves of a SIsFS structure with the same PdFe metal don't demonstrate these irregularities $[15,16]$.

To characterize a junction's stability with respect to $H$ variations it is convenient to introduce the parameter $\eta=\left(d J_{C} / J_{C}\right) /(d H / H)$ which relates the relative change in the critical current to the relative change in the exchange energy. The larger the magnitude of $\eta$ the more intense irregularities in a SFS junction are expected with variation of $H$. In Fig.2.8 we compare SIsFS devices with conventional SFS, SIFS, and SIFIS junctions making use of two of the most important parameters: the instability parameter $\eta$ and the product $J_{C} R_{N}$, the value of which characterizes high-frequency properties of the structures. The calculations have been done in the frame of the Usadel equations for the same set of 


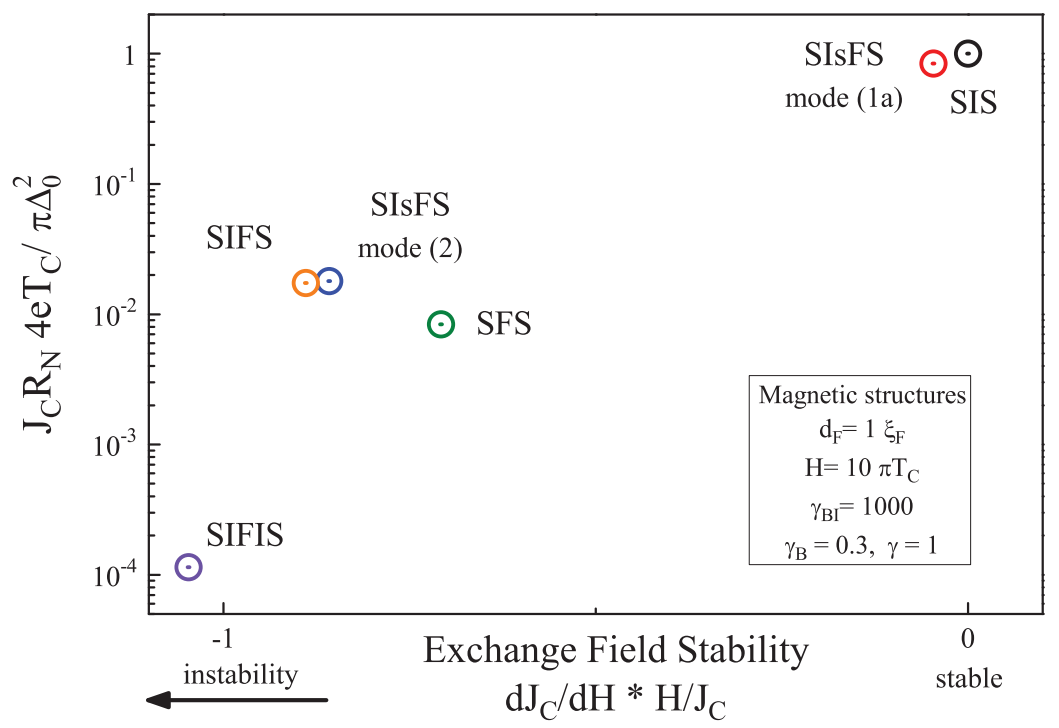

Figure 2.8. Comparison of different types of Josephson structures, marked by points on the phase plane, in terms of $J_{C} R_{N}$ and exchange field stability $\eta$. All calculation have been performed for $T=0.5 T_{C}, d_{F}=\xi_{F}, \gamma_{B I}=1000, \gamma_{B}=0.3, \gamma=1$. For SIsFS structures $d_{s}=5 \xi_{S}$ and $d_{s}=0.5 \xi_{S}$ are taken in mode (1a) and mode (2), respectively.

junction parameters, namely $T=0.5 T_{C}, H=10 \pi T_{C}, d_{F}=\xi_{F}, \gamma_{B I}=1000, \gamma_{B}=0.3$, $\gamma=1$.

It can be seen that the presence of two tunnel barriers in a SIFIS junction results in the smallest $J_{C} R_{N}$ and strong instability. The SIFS and SIsFS structures in mode (2) demonstrate better results with almost the same parameters. Conventional SFS structures have a twice smaller $J_{C} R_{N}$ product, having a higher critical current but lower resistivity. At the same time, SFS junctions are more stable due to the lack of a low-transparent tunnel barrier. The latter is the main source of instability due to sharp phase discontinuities at the barrier 'I'.

In contrast to the standard SFS, SIFS and SIFIS junctions, SIsFS structures achieve $J_{C} R_{N}$ and stability characteristics comparable to those of SIS tunnel junctions. This unique property is favorable for application of SIsFS structures in superconducting electronic circuits. 


\subsection{Appendix}

\subsubsection{Boundary problem at $T \lesssim T_{C}$}

In the limit of high temperature

$$
G_{S}=G_{s}=G_{F}=\operatorname{sgn}(\Omega)
$$

and the boundary problem reduces to to the system of linerized equations. Their solution in the $\mathrm{F}$ layer, $\left(0 \leq x \leq d_{F}\right)$, has the form

$$
\Phi_{F}=C \sinh \frac{\sqrt{\Theta}\left(x-d_{F} / 2\right)}{\xi_{F}}+D \cosh \frac{\sqrt{\Theta}\left(x-d_{F} / 2\right)}{\xi_{F}},
$$

where $\Theta=\widetilde{\Omega} \operatorname{sgn}(\Omega)$. For transparent FS and sF interfaces $\left(\gamma_{B}=0\right)$ from the boundary conditions (2.6), (2.7) and (2.39) it is easy to get that

$$
\begin{gathered}
\frac{\xi_{s}}{\gamma \sqrt{\Theta}} \frac{d}{d x} \Phi_{s}(0)=-\Phi_{s}(0) \operatorname{coth} \frac{d_{F} \sqrt{\Theta}}{\xi_{F}}+\frac{\Phi_{S}\left(d_{F}\right)}{\sinh \frac{d_{F} \sqrt{\Theta}}{\xi_{F}}} \\
\frac{\xi_{S}}{\gamma \sqrt{\Theta}} \frac{d}{d x} \Phi_{S}\left(d_{F}\right)=\Phi_{S}\left(d_{F}\right) \operatorname{coth} \frac{d_{F} \sqrt{\Theta}}{\xi_{F}}-\frac{\Phi_{s}(0)}{\sinh \frac{d_{F} \sqrt{\Theta}}{\xi_{F}}}
\end{gathered}
$$

and thus reduce the problem to the solution of Ginzburg-Landau (GL) equations in s and S films.

$$
\begin{gathered}
\xi_{S}^{2}(T) \frac{d^{2}}{d x^{2}} \Delta_{k}-\Delta_{k}\left(\Delta_{0}^{2}-\left|\Delta_{k}\right|^{2}\right)=0, \Delta_{0}^{2}=\frac{8 \pi^{2} T_{C}\left(T_{C}-T\right)}{7 \zeta(3)}, \\
J=\frac{J_{G}}{\Delta_{0}^{2}} \operatorname{Im}\left(\Delta_{k}^{*} \xi_{S}(T) \frac{d}{d x} \Delta_{k}\right), J_{G}=\frac{\pi \Delta_{0}^{2}}{4 e \rho_{S} T_{C} \xi_{S}(T)},
\end{gathered}
$$

where $\xi_{S}(T)=\pi \xi_{S} / 2 \sqrt{1-T / T_{C}}$ is GL coherence length and $k$ equals to $s$ or $S$ for $-d_{s} \leq$ $x \leq 0$ and $x \geq d_{F}$, respectively. At Is, $\mathrm{sF}$ and FS interfaces GL equations should be supplemented by the boundary conditions in the form [37]

$$
\begin{gathered}
\xi_{S}(T) \frac{d}{d x} \Delta_{k}(z)=b(z) \Delta_{k}(z), b(z)=\frac{\Sigma_{1}(z)}{\Sigma_{2}(z)}, \\
\Sigma_{1}(z)=\sum_{\omega=-\infty}^{\infty} \xi_{S}(T) \frac{d}{d x} \frac{\Phi_{k}(z)}{\Omega^{2}}, \Sigma_{2}(z)=\sum_{\omega=-\infty}^{\infty} \frac{\Phi_{k}(z)}{\Omega^{2}},
\end{gathered}
$$


where $z=-d_{s}, 0, d_{F}$. In typical experimental situation $\gamma_{B I} \gg 1, \gamma \sqrt{H} \gg 1$ and $d_{F} \sqrt{H} \gtrsim$ $\xi_{F}$. In this case in the first approximation

$$
\Phi_{S}\left(d_{F}\right)=0, \Phi_{s}(0)=0, \frac{d}{d x} \Phi_{s}\left(-d_{s}\right)=0
$$

and in the vicinity of interfaces

$$
\begin{gathered}
\Phi_{S}(x)=\Delta_{S}(x)=B_{S} \frac{\left(x-d_{F}\right)}{\xi_{S}(T)}, d_{F} \lesssim x \ll \xi_{S}(T), \\
\Phi_{s}(x)=\Delta_{s}(x)=-B_{s} \frac{x}{\xi_{s}(T)},-\xi_{S}(T) \ll x \lesssim 0, \\
\Phi_{s}(x)=\Delta_{s}(x)=\Delta_{s}\left(-d_{s}\right),-d_{s} \lesssim x \ll-d_{s}+\xi_{S}(T),
\end{gathered}
$$

where $B_{S}, B_{s}$, and $\Delta_{s}\left(-d_{s}\right)$ are independent on $x$ constants. Substitution of the solutions (2.46) - (2.48) into (2.15), (2.40), (2.41) gives

$$
\begin{gathered}
\Gamma_{B I} \xi_{S}(T) \frac{d}{d x} \Phi_{s}\left(-d_{s}\right)=\Delta_{s}\left(-d_{s}\right)-\Delta_{0}, \\
\Phi_{S}\left(d_{F}\right)=\frac{B_{s}}{\Gamma \sqrt{\Theta} \sinh \frac{d_{F} \sqrt{\widetilde{\Omega}}}{\xi_{F}}}+\frac{B_{S} \cosh \frac{d_{F} \sqrt{\Theta}}{\xi_{F}}}{\Gamma \sqrt{\Theta} \sinh \frac{d_{F} \sqrt{\Theta}}{\xi_{F}}}, \\
\Phi_{s}(0)=\frac{B_{s} \cosh \frac{d_{F} \sqrt{\Theta}}{\xi_{F}}}{\Gamma \sqrt{\widetilde{\Omega}} \sinh \frac{d_{F} \sqrt{\Theta}}{\xi_{F}}}+\frac{B_{S}}{\Gamma \sqrt{\Theta} \sinh \frac{d_{F} \sqrt{\Theta}}{\xi_{F}}}, \\
\Gamma_{B I}=\frac{\gamma_{B I} \xi_{S}}{\xi_{s}(T)}, \Gamma=\frac{\gamma_{B I} \xi_{s}(T)}{\xi_{S}}
\end{gathered}
$$

From definition (2.44), (2.45) of coefficients $b(z)$ and expressions (2.49) - (2.51) it follows that

$$
\begin{gathered}
\Gamma_{B I} \xi_{s}(T) \frac{d}{d x} \Delta_{s}\left(-d_{s}\right)=-\left(\Delta_{0}-\Delta_{s}\left(-d_{s}\right)\right), \\
\xi_{s}(T) \frac{d}{d x} \Delta_{s}(0)=-\frac{q+p}{2} \Gamma \Delta_{s}(0)-\frac{q-p}{2} \Gamma \Delta_{S}\left(d_{F}\right), \\
\xi_{S}(T) \frac{d}{d x} \Delta_{S}\left(d_{F}\right)=\frac{q+p}{2} \Gamma \Delta_{S}\left(d_{F}\right)+\frac{q-p}{2} \Gamma \Delta_{s}(0),
\end{gathered}
$$

where

$$
\begin{aligned}
p^{-1} & =\frac{8}{\pi^{2}} R e \sum_{\omega=0}^{\infty} \frac{1}{\Omega^{2} \sqrt{\widetilde{\Omega}} \operatorname{coth} \frac{d_{F} \sqrt{\widetilde{\Omega}}}{2 \xi_{F}}}, \\
q^{-1} & =\frac{8}{\pi^{2}} R e \sum_{\omega=0}^{\infty} \frac{1}{\Omega^{2} \sqrt{\widetilde{\Omega}} \tanh \frac{d_{F} \sqrt{\widetilde{\Omega}}}{2 \xi_{F}}} .
\end{aligned}
$$


In considered limit both suppression parameters $\Gamma_{B I} \gg 1$ and $\Gamma \gg 1$ are large and from relations $(2.15),(2.40),(2.41)$ in the first approximation on these parameters we get that the boundary conditions $(2.53)-(2.55)$ can be simplified to

$$
\xi_{S}(T) \frac{d}{d x} \Delta_{s}\left(-d_{s}\right)=0, \Delta_{s}(0)=0, \Delta_{S}\left(d_{F}\right)=0 .
$$

Taking into account that in this approximation supercurrent $j=0$ and $\Delta_{S}(\infty)=\Delta_{0}$ from (2.42), (2.58) it follows that

$$
\Delta_{S}(x)=\delta_{S}(x) \exp \{i \varphi\}, \delta_{S}(x)=\Delta_{0} \tanh \frac{x-d_{F}}{\sqrt{2} \xi_{S}(T)}
$$

while

$$
\Delta_{s}(x)=\delta_{s}(x) \exp \{i \chi\},
$$

where $\delta_{s}(x)$ is the solution of transcendental equation

$$
F\left(\frac{\delta_{s}(x)}{\delta_{s}\left(-d_{s}\right)}, \frac{\delta_{s}\left(-d_{s}\right)}{\Delta_{0} \eta}\right)=-\frac{x \eta}{\sqrt{2} \xi_{s}(T)}, \eta=\sqrt{2-\frac{\delta_{s}^{2}\left(-d_{s}\right)}{\Delta_{0}^{2}}}
$$

and $\delta_{s}\left(-d_{s}\right)$ is a solution of the same equation at the SIs boundary $x=-d_{s}$

$$
K\left(\frac{\delta_{s}\left(-d_{s}\right)}{\Delta_{0} \eta}\right)=\frac{d_{s} \eta}{\sqrt{2} \xi_{s}(T)} .
$$

Here $F(y, z)$ and $K(z)$ are the incomplete and complete elliptic integral of the first kind respectively.

Substitution of (2.59), (2.60) into (2.53) - (2.55) gives that in the next approximation on $\Gamma_{B I}^{-1}$ and $\Gamma^{-1}$

$$
\begin{gathered}
J\left(-d_{s}\right)=J_{G} \frac{\delta_{s}\left(-d_{s}\right)}{\Gamma_{B I} \Delta_{0}} \sin (\chi) \\
J(0)=J\left(d_{F}\right)=J_{G} \frac{\Gamma(p-q)}{2 \Delta_{0}^{2}} \delta_{s}(0) \delta_{S}\left(d_{F}\right) \sin (\varphi-\chi),
\end{gathered}
$$

where

$$
\begin{aligned}
& \delta_{s}(0)=-\frac{2 b(q-p) \cos (\varphi-\chi)+2 a(q+p)}{\Gamma\left[(q+p)^{2}-(q-p)^{2} \cos ^{2}(\varphi-\chi)\right]}, \\
& \delta_{S}\left(d_{F}\right)=\frac{2 b(q+p)+2 a(q-p) \cos (\varphi-\chi)}{\Gamma\left((q+p)^{2}-(q-p)^{2} \cos ^{2}(\varphi-\chi)\right)},
\end{aligned}
$$


are magnitudes of the order parameters at the FS interfaces and

$$
a=-\delta_{s}\left(-d_{s}\right) \sqrt{1-\frac{\delta_{s}^{2}\left(-d_{s}\right)}{2 \Delta_{0}^{2}}}, b=\frac{\Delta_{0}}{\sqrt{2}}
$$

Phase, $\chi$, of the order parameters of the s layer is determined from equality of currents (2.63), (2.64). 


\section{Bibliography}

[1] A. A. Golubov, M. Yu. Kupriyanov, E. Il'ichev, Rev. Mod. Phys. 76, 411 (2004).

[2] A. I. Buzdin, Rev. Mod. Phys. 77, 935 (2005).

[3] F. S. Bergeret, A. F. Volkov, K. B. Efetov, Rev. Mod. Phys. 77, 1321 (2005).

[4] S. Oh, D. Youm, and M. Beasley, Appl. Phys. Lett. 71 2376, (1997).

[5] R. Held, J. Xu, A. Schmehl, C.W. Schneider, J. Mannhart, and M. Beasley, Appl. Phys. Lett. 89, 163509 (2006).

[6] C. Bell, G. Burnell, C. W. Leung, E. J. Tarte, D.-J. Kang, and M. G. Blamire, Appl. Phys. Lett. 84, 1153 (2004).

[7] E. Goldobin , H. Sickinger, M. Weides, N. Ruppelt, H. Kohlstedt, R. Kleiner , D. Koelle, arXiv:1306.1683 (2013).

[8] V. V. Bol'ginov, V. S. Stolyarov, D. S. Sobanin, A. L. Karpovich, and V. V. Ryazanov, Pis'ma v ZhETF 95, 408 (2012) [JETP Lett. 95366 (2012)].

[9] V. V. Ryazanov, V. V. Bol'ginov, D. S. Sobanin, I. V. Vernik, S. K. Tolpygo, A. M. Kadin, O. A. Mukhanov, Physics Procedia 36, 35 (2012).

[10] T. I. Larkin, V. V. Bol'ginov, V. S. Stolyarov, V. V. Ryazanov, I. V. Vernik, S. K. Tolpygo, and O. A. Mukhanov, Appl. Phys. Lett. 100, 222601 (2012).

[11] I. V. Vernik, V. V. Bol'ginov, S. V. Bakurskiy, A. A. Golubov, M. Yu. Kupriyanov, V. V. Ryazanov and O. A. Mukhanov, IEEE Tran. Appl. Supercond., 23, 1701208 (2013).

[12] S. V. Bakurskiy, N. V. Klenov, I. I. Soloviev, V. V. Bol'ginov, V. V. Ryazanov, I. I. Vernik, O. A. Mukhanov, M. Yu. Kupriyanov, and A. A. Golubov, Appl. Phys. Lett. 102, 192603 (2013).

[13] T. Ortlepp, Ariando, O. Mielke, C. J. M. Verwijs, K. F. K. Foo, H. Rogalla, F. H. Uhlmann, and H. Hilgenkamp, Science 312, 1495 (2006). 
[14] V. Ryazanov, Uspechi Fizicheskich Nauk 169, 920 (1999) [Physics-Uspekhi 42, 825 (1999)].

[15] A.K. Feofanov, V.A. Oboznov, V.V. Bol'ginov, et. al., Nature Physics 6, 593 (2010).

[16] A. V. Ustinov and V. K. Kaplunenko, J. Appl. Phys. 94, 5405 (2003).

[17] A. Buzdin and A. E. Koshelev, Phys. Rev. B 67, 220504(R) (2003).

[18] A. E. Koshelev, Phys. Rev. B 86, 214502 (2012).

[19] N. G. Pugach, E. Goldobin, R. Kleiner, and D. Koelle, Phys. Rev. B 81, 104513 (2010).

[20] E. Goldobin, D. Koelle, R. Kleiner, and R.G. Mints, Phys. Rev. Lett, 107, 227001 (2011).

[21] H. Sickinger, A. Lipman, M Weides, R.G. Mints, H. Kohlstedt, D. Koelle, R. Kleiner, and E. Goldobin, Phys. Rev. Lett, 109, 107002 (2012).

[22] S. V. Bakurskiy, N. V. Klenov, T. Yu. Karminskaya, M. Yu. Kupriyanov, and A. A. Golubov, Supercond. Sci. Technol. 26, 015005 (2013).

[23] D. M. Heim, N. G. Pugach, M. Yu. Kupriyanov, E. Goldobin, D. Koelle, and R. Kleiner, J. Phys.Cond. Mat. 25, 215701 (2013).

[24] M. Alidoust, J. Linder, Phys. Rev. B 87, 060503 (2013).

[25] Jun-Feng Liu, K.S. Chan, Phys. Rev. B 82, 184533 (2010).

[26] V. V. Ryazanov, V. A. Oboznov, A. Yu. Rusanov, A. V. Veretennikov, A. A. Golubov, and J. Aarts, Phys. Rev. Lett. 86, 2427 (2001).

[27] V. A. Oboznov, V. V. Bol'ginov, A. K. Feofanov, V. V. Ryazanov, and A. I. Buzdin, Phys. Rev. Lett. 96, 197003 (2006).

[28] T. Kontos, M. Aprili, J. Lesueur, F. Genet, B. Stephanidis, and R. Boursier, Phys. Rev. Lett. 89, 137007 (2002).

[29] M. Weides, M. Kemmler, H. Kohlstedt, A. Buzdin, E. Goldobin, D. Koelle, R. Kleiner, Appl. Phys. Lett. 89, 122511 (2006). 
[30] M. Weides, M. Kemmler, H. Kohlstedt, R. Waser, D. Koelle, R. Kleiner, and E. Goldobin Physical Review Letters 97, 247001 (2006).

[31] F. Born, M. Siegel, E. K. Hollmann, H. Braak, A. A. Golubov, D. Yu. Gusakova, and M. Yu. Kupriyanov, Phys. Rev. B. 74, 140501 (2006).

[32] J. Pfeiffer, M. Kemmler, D. Koelle, R. Kleiner, E. Goldobin, M. Weides, A. K. Feofanov, J. Lisenfeld, and A. V. Ustinov, Physical Review B 77, 214506 (2008).

[33] A. S. Vasenko, A. A. Golubov, M. Yu. Kupriyanov, and M. Weides, Phys.Rev.B, 77, 134507 (2008).

[34] A. S. Vasenko, S. Kawabata, A. A. Golubov, M. Yu. Kupriyanov, C. Lacroix, F. W. J. Hekking, Phys. Rev. B 84, 024524 (2011).

[35] K. D. Usadel, Phys. Rev. Lett. 25, 507 (1970).

[36] M. Yu. Kuprianov and V. F. Lukichev, Zh. Eksp. Teor. Fiz. 94, 139 (1988) [Sov. Phys. JETP 67, 1163 (1988)].

[37] Z. G.Ivanov, M. Yu. Kupriyanov, K. K. Likharev, S. V. Meriakri, and O. V. Snigirev, Fiz. Nizk. Temp. 7, 560 (1981). [Sov. J. Low Temp. Phys. 7, 274 (1981)].

[38] A. A.Zubkov, and M. Yu. Kupriyanov, Fiz. Nizk. Temp. 9, 548 (1983) [Sov. J. Low Temp. Phys. 9, 279 (1983)].

[39] M. Yu. Kupriyanov, Pis'ma v ZhETF 56, 414 (1992) [JETP Lett. 56, 399 (1992)].

[40] A.A. Golubov and M.Y. Kupriyanov, Pis'ma v ZhETF 81, 419 (2005) [JETP Lett. 81, $335(2005)]$.

[41] A.A. Golubov, M.Y. Kupriyanov, and Y.V. Fominov,.Pis'ma v ZhETF 75, 709 (2002). [JETP Lett. 75, 588 (2002)].

[42] R. Meservey and P. M. Tedrow, Phys. Rep. 238, 173 (1994).

[43] E. A. Koshina, V. N. Krivoruchko, Metallofizika i Noveishie Tekhnologii, 35, 45 (2013).

[44] L. S. Uspenskaya, A. L. Rakhmanov, L. A. Dorosinskiy, A. A. Chugunov, V. S. Stolyarov, O. V. Skryabina, and S. V. Egorov, Pis'ma v ZhETF 97, 176 (2013) [JETP Lett. 97, 155 (2013)]. 
[45] F. S. Bergeret, A. F. Volkov, and K. B. Efetov, Phys. Rev.Lett. 86, 3140 (2001).

[46] Ya. V. Fominov, N. M. Chtchelkatchev, and A. A. Golubov, Phys. Rev. B 66, 014507 (2002).

[47] T. Yu. Karminskaya and M. Yu. Kupriyanov, Pis'ma v ZhETF 85, 343 (2007) [JETP Lett. 85, 286 (2007)].

[48] T. E. Golikova, F. Hübler, D. Beckmann, I. E. Batov, T. Yu. Karminskaya, M. Yu. Kupriyanov, A. A. Golubov and V. V. Ryazanov, Phys. Rev. B 86, 064416 (2012). 


\section{Chapter 3}

\section{Comparison of theory for SIsFS junctions with experimental data}

\subsection{Introduction}

Practical applications of superconducting digital circuits were significantly limited by the relatively low capacity of superconducting memory. This motivated initial proposals to use superconductor/ferromagnet (S/F) hybrid structures as basis for the development in cryogenic magnetic Random Access Memories (RAMs) [1-3]. Following the first experimental realization of SFS Josephson junctions [4,5], much attention was paid to realize Josephson devices with complex magnetic barriers allowing switching between high and low critical currents. A number of different device structures were considered [6-14] based either on superconducting spintronics effects or on singlet-triplet switching within the magnetic barrier. However, these approaches were based on structures with reduced characteristic voltage $I_{c} R_{N}$ of junctions.

Recently, successful realization of switchable $\mathrm{Nb}-\mathrm{Al} / \mathrm{AlO}_{x}-\mathrm{Nb}-\mathrm{Pd}_{0.99} \mathrm{Fe}_{0.01}-\mathrm{Nb}$ junctions was reported in [15-17]. These junctions are of SIsFS type, i.e., a serial connection of the SIs tunnel junction and sFS sandwich. SIsFS structure has high characteristic voltage, $I_{c} R_{N}$, due to the presence of tunnel barrier 'I'. At the same time the whole structure behaves as a single junction with respect to an external magnetic field, $H_{\text {ext }}$, since intermediate layer $\mathrm{s}$ is too thin to screen $H_{\text {ext }}$. As a result, the magnetic field entering the $\mathrm{Pd}_{0.99} \mathrm{Fe}_{0.01}$ layer will modify its effective magnetization, facilitating the critical current control. According to Ref. [18], effective magnetization in the dilute $\mathrm{Pd}_{0.99} \mathrm{Fe}_{0.01}$ is controlled by Fe-rich nano-clusters which can be easily reordered by a weak magnetic field. The purpose of this paper is to develop a theory describing various modes of operation of SIsFS Josephson devices. We compare the results with experimental data recently obtained for for $\mathrm{Nb}-\mathrm{Al} / \mathrm{AlO}_{x}-\mathrm{Nb}-\mathrm{Pd}_{0.99} \mathrm{Fe}_{0.01}-\mathrm{Nb}$ junctions.

We consider the multilayered structure presented in Fig. 1. It consists of two super-

\footnotetext{
${ }^{0}$ The experimental data used in this chapter are provided by the groups from HYPRES (USA) and ISSP RAS (Chernogolovka, Russia)
} 


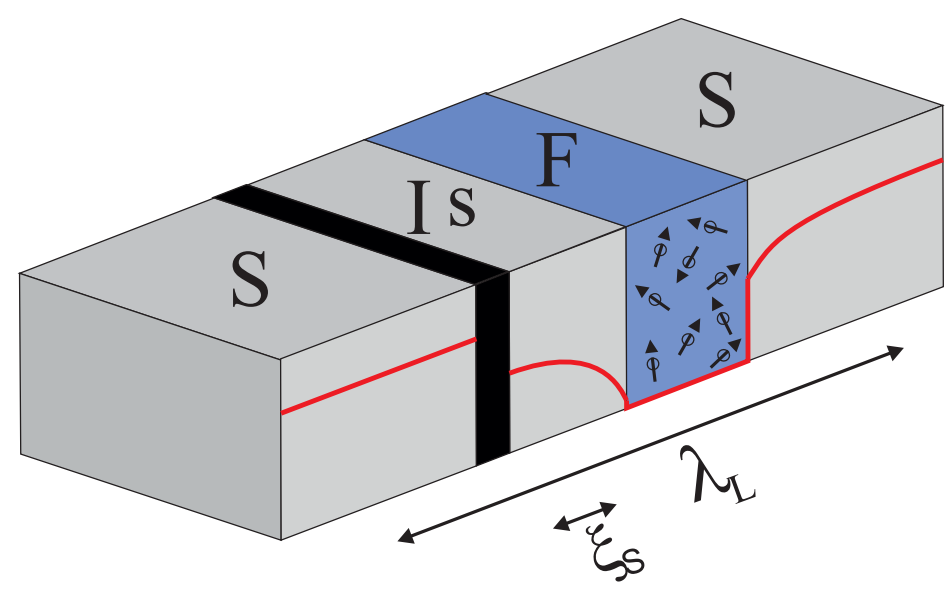

Figure 3.1. Schematic design of SIsFS Josephson junction. Solid line demonstrates typical distribution of pair potential $\Delta$ along the structure. It reaches bulk values in the $\mathrm{S}$ electrodes, is suppressed at middle s layer and disappears in ferromagnetic F area. Also at the figure the typical scales of characteristic length are marked: $\lambda_{L}$ is London penetration depth and $\xi_{S}$ is a coherence length actual for Niobium.

conducting electrodes 'S' separated by a tunnel barrier 'I', an intermediate thin superconducting film 's' and a ferromagnetic layer ' $F$ '. To describe the supercurrent transport in the structure we assume that the conditions of a dirty limit are fulfilled for all metals. We also assume that all superconducting films in the structure are made from identical materials, i.e., they can be described by the same critical temperature, $T_{C}$, and coherence length, $\xi_{S}=\left(D_{S} / 2 \pi T_{C}\right)^{1 / 2}$, where $D_{S}$ is the electronic diffusion coefficient. The tunnel barrier I and the $\mathrm{sF}$ and FS interfaces are characterized, respectively, by the following parameters $\gamma_{B I}=R_{I} \mathcal{A} / \xi_{S} \rho_{S}, \gamma_{B F S}=R_{F S} \mathcal{A} / \xi_{F} \rho_{F}$, and $\gamma=\rho_{S} \xi_{S} / \xi_{F} \rho_{F}$. Here $\mathcal{A}, R_{B I}$ and $R_{B S F}$ are the area and the resistances of the interfaces, $\xi_{S, F}$ and $\rho_{S, F}$ are the coherence lengths and normal state resistivities of $\mathrm{S}$ and $\mathrm{F}$ materials, respectively. Under the above conditions the Josephson effect in the SIsFS junctions can be described by solving the Usadel equations [19-22] with Kupriyanov-Lukichev (KL) boundary conditions [23] at Is, sF and FS interfaces and with the bulk pair potential in the depth of S-electrodes.

The formulated above boundary problem has been solved numerically. The results are summarized in Figs.2-4, where various modes of operation of the structure are defined according to chosen materials and layer thicknesses. These modes are clearly defined by the dependencies of characteristic voltage $I_{C} R_{N}$ on thickness of superconductor, $L_{s}$, and ferromagnetic, $L_{F}$, layers (see Fig.3.2 - Fig.3.4). 


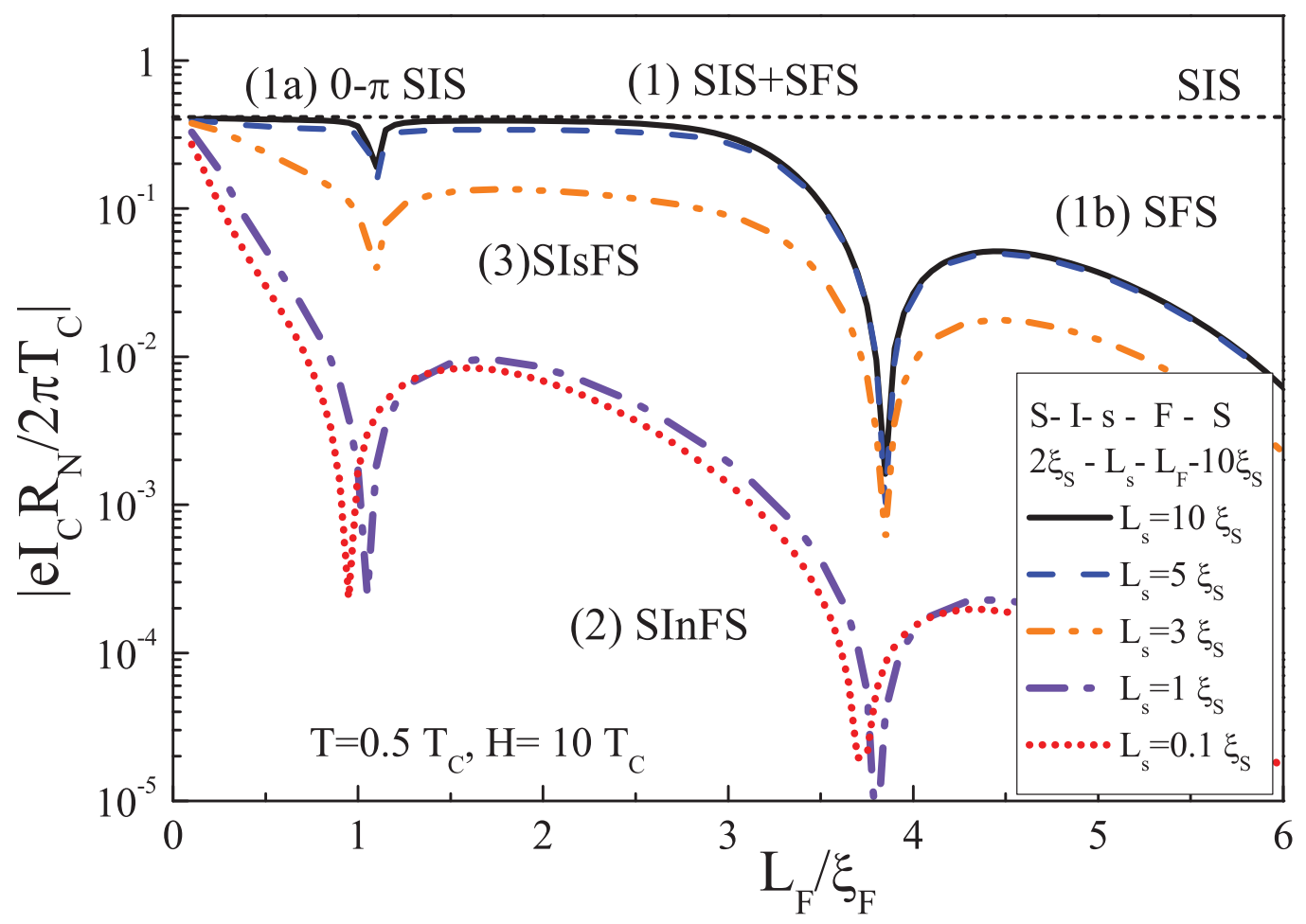

Figure 3.2. Characteristic voltage $I_{C} R_{N}$ of the SIsFS structures versus thickness of the Flayer $L_{F}$ for different thicknesses of the middle superconducting film $L_{s}$ at $T=0.5 T_{C}$. Shortdashed line shows the $I_{C} R_{N}$ product of the tunnel SIS junction at the same temperature. Interface parameters: $\gamma_{B I}=1000, \gamma_{B F S}=0.3$ and $\gamma=1$ at the $\mathrm{sF}$ and FS interfaces.

\subsection{Temperature dependence}

Mode (1) in Fig.2. If the thickness of the middle s-electrode, $L_{s}$, is much larger then the critical thickness of s layer, $L_{s C}$, which separates the different modes of operation in SIsFS structures, the pair potential $\Delta$ in the s layer is close to that of bulk material. Note that the critical thickness $L_{s C}$ in an $\mathrm{sN}(\mathrm{sF}$ ) bilayer at a given temperature is generally defined as a minimal thickness of an s-layer when superconductivity still exists. In the mode 1 the structure can be considered as a pair of SIs and sFS junctions connected in series. Therefore, the properties of the structure in parameter range (1) are almost independent on the thickness $L_{s}$ and are determined by the junction with smallest critical current. It is seen from Fig.3.2 that for the given parameter set, $T=0.5 T_{C}, H=10 T_{C}, \gamma=1, \gamma_{B I}=1000$, $\gamma_{B F S}=0.3$, the critical thickness of the s layer, $L_{s C}$, is close to $3 \xi_{S}$.

Mode (1a) in Fig.3.2. In the ordinary case of $I_{C_{-} S I s} \ll I_{C_{-} s F S}$ the behavior of the structure coincides with that of conventional SIS junction with one important distinction - the sFS junction can turn the SIsFS structure into a $\pi$-state. At the same time, other 
properties like high $I_{C} R_{N}$ product and sinusoidal current-phase relation are preserved in the $\pi$-state. Therefore the structure can be called switchable 0- $\pi$ SIS junction.

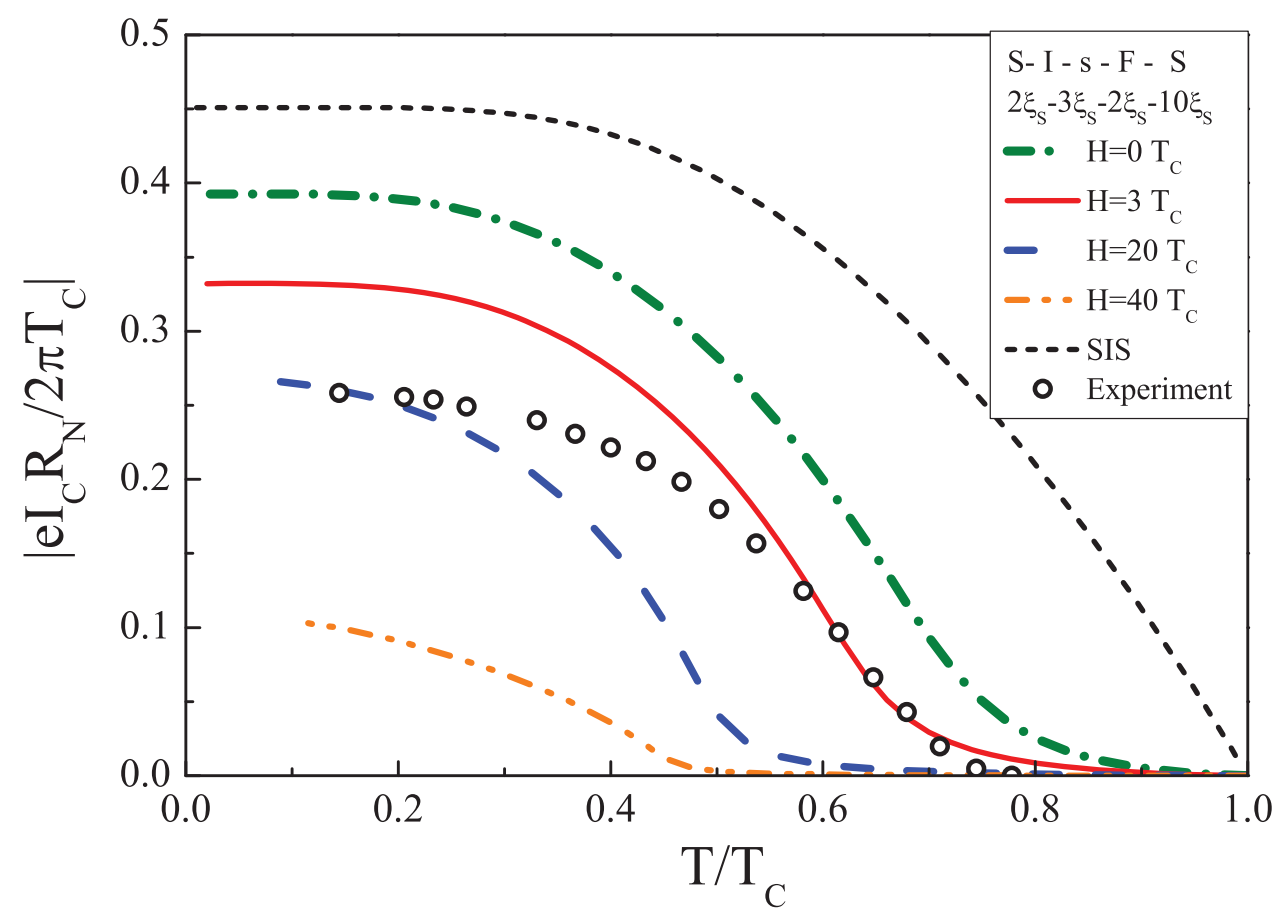

Figure 3.3. The temperature dependence of $I_{C} R_{N}$ of SIsFS structure for different values of exchange field $H$ in the F-layer. The dashed line demonstrates the dependence characteristic for a conventional tunnel SIS Junction. It is seen how the exchange field $\mathrm{H}$ shifts the effective critical temperature $T_{C}^{*}$, corresponding to the switching of the s-layer from the superconducting state to the normal one. The circles show $I_{C} R_{N}$ measured in $\mathrm{Nb}-\mathrm{Al} / \mathrm{AlO}_{x^{-}}$ $\mathrm{Nb}-\mathrm{Pd}_{0.99} \mathrm{Fe}_{0.01}-\mathrm{Nb}$ junctions [16], proving the existence of effective critical temperature $T_{C}^{*}$ in these samples.

Mode (1b) in Fig.3.2. Another limiting case is realized for large $L_{F}$ values and high exchange fields $H$. Namely, the structure transforms into a standard SFS-junction without any influence of tunnel barrier.

Mode (2) in Fig.3.2. The absence of superconductivity in the s-electrode in the opposite case $\left(L_{s} \ll L_{s C}\right)$ leads to formation of the complex -InF- weak link area, where $\mathrm{n}$ marks the intermediate $\mathrm{s}$ film in the normal state. It results in much smaller critical current value $I_{C}$, with the magnitude close to that in well-known SIFS junctions [24]. The dependence of $I_{C}$ on the thickness $L_{s}$ is weak due to large decay length in the n-region with suppressed superconductivity.

Mode (3) in Fig.3.2. Conversely, in the intermediate case $\left(L_{s} \approx L_{s C}\right)$ the properties of the structure are extra sensitive to variations of the decay lengths parameters. Within 
the considered intermediate thickness range the system may transform from the mode (1) to the mode (2). Moreover, in this situation the system is sensitive to the F-layer parameters (thickness $L_{F}$ and exchange field $H$ ) since these parameters control the suppression of superconductivity in the $\mathrm{sF}$ bilayer.

This sensitivity allows one to change an operation mode by changing the parameters such as effective exchange field $H$ and temperature $T$. Note, that depending on the domain structure of a ferromagnet and morphology of the F-film it might be possible to control the effective exchange field $H$.

Fig.3.3 demonstrates the temperature dependence of the critical current in the structures with thickness around critical one $\left(L_{s C}=3 \xi_{S}\right)$ for different values of exchange field $H$. These structures are characterized by the existence of the effective critical temperature $T_{C}^{*}$ which corresponds to the appearance of superconductivity in middle s-layer and, correspondingly, to an exponential growth of the current. Therefore, $T_{C}^{*}$ may significantly shift during remagnetization of the system (due to changing of $\mathrm{H}$, as pointed out above). Thus, system can exist either in the superconducting or in the normal state depending on the history of the application of a magnetic field. On the other hand, from point of view of practical applications, the 0- $\pi$ SIS mode (1a) seems more relevant.

Fig.3.4 demonstrates that change of F-layer thickness $L_{F}$ leads to $0-\pi$ transition. The system can be switched into a $\pi$-state keeping the value of $I_{C} R_{N}$ product, i.e., Josephson frequency, on the level characteristic for tunnel SIS junctions. Moreover, it should be noted that this property of the considered structure is unique. In the conventional SFS devices in order to reach the $\pi$-state it is necessary to realize either $L_{F} \gtrsim(2-3) \xi_{F}$ or very large values of the $\gamma_{B F S} \gg 1$ parameter at the SF interfaces. In both cases the $I_{C} R_{N}$ product in the $\pi$-state is strongly reduced $[20-22,24]$.

\subsection{External magnetic field}

In the parameter range when SIsFS junction is far from to $0-\pi$ transition, currentphase relation has a sinusoidal form, $I(\varphi) \approx I_{S I s} \sin \varphi$. To calculate the dependence of $I_{C}$ from external magnetic field, $H_{e x t}$, we may use the standard Fraunhofer expression,

$$
I_{C}\left(H_{e x t}\right)=I_{C}(0)\left|\frac{\sin \left(\pi \Phi / \Phi_{0}\right)}{\pi \Phi / \Phi_{0}}\right|
$$




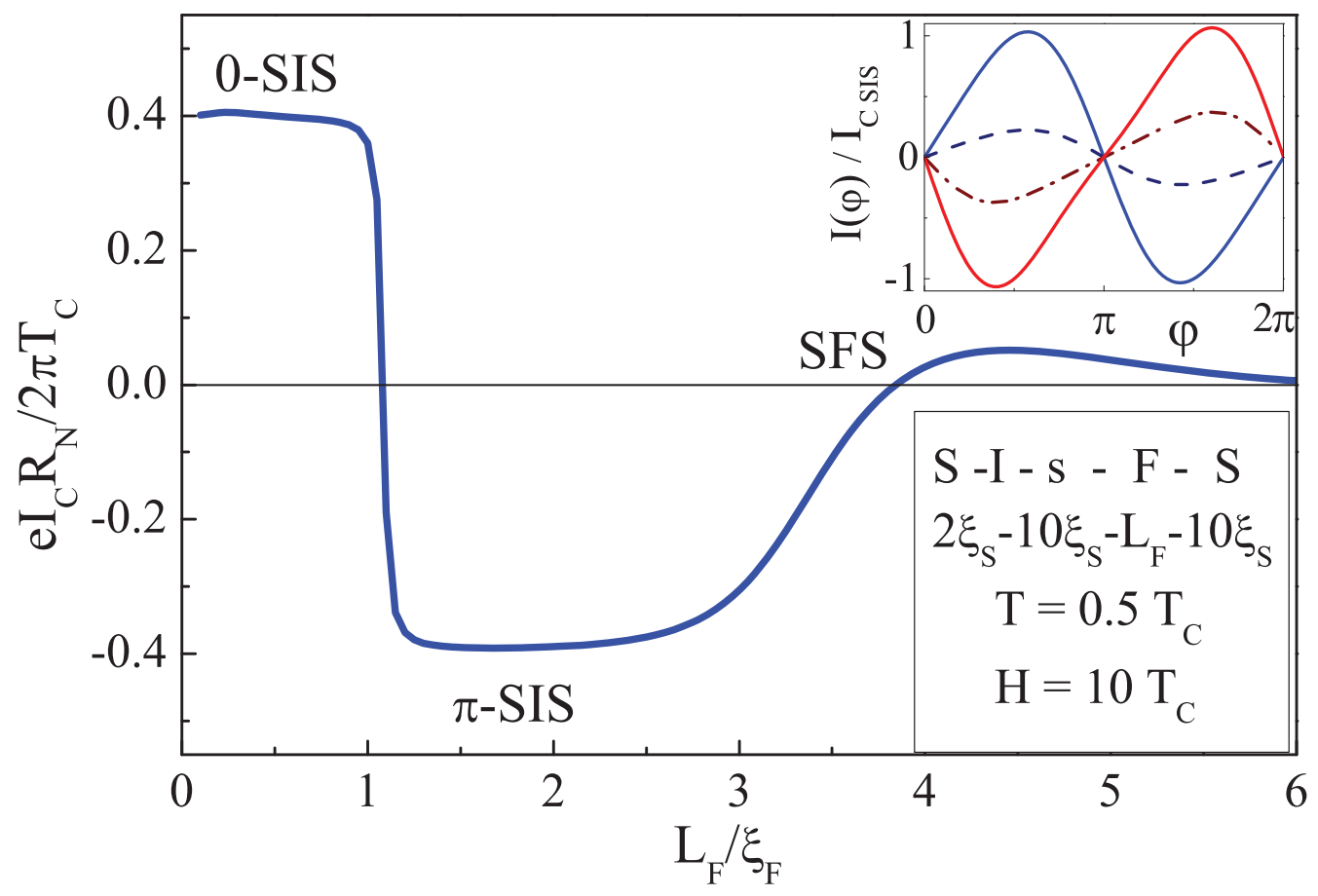

Figure 3.4. The dependence of supercurrent $I_{C} R_{N}$ on F-layer thickness $L_{F}$ in the SIsFS structure with the s-layer in the superconducting state. Inset shows the current-phase relation in the vicinity of the first $0-\pi$ transition. Switching from 0 to $\pi$ state in the mode (1a) conserves the value of critical current $I_{S}$ as well as characteristic voltage $I_{C} R_{N}$.

where

$$
\Phi=W\left|L_{e f f} H_{e x t}+L_{F} H_{0} N\left(n_{\uparrow}-n_{\downarrow}\right)\right|
$$

is magnetic flux inside of SIsFS junction, $\Phi_{0}$ is flux quantum, $L_{e f f}=2 \lambda_{L}+L_{s}+L_{F}+L_{I}$, $\lambda_{L}$ is London penetration depth of $\mathrm{S}$ electrodes, $L_{I}$ is thickness of I layer, $N$ is the full number of clusters, $n_{\uparrow, \downarrow}=N_{\uparrow, \downarrow} / N$ are the concentrations of clusters in the F layer oriented parallel $\left(N_{\uparrow}\right)$ or antiparallel $\left(N_{\downarrow}\right)$ to the direction of $H_{\text {ext }}$ in the saturation region and $H_{0}$ is an average magnetic field generated by a single magnetic cluster. In our simple model, $H_{0}$ is assumed to be a constant, while $n_{\uparrow}$ and $n_{\downarrow}$ are functions of $H_{\text {ext }}$. We assume further that the probability density $p\left(H_{\text {ext }}\right)$ of a flip of a cluster in $H_{e x t}$ is described by a Gaussian distribution

$$
p\left(H_{e x t}\right)=(\sqrt{2} / \sqrt{\pi} \delta) \exp \left[-\left(H_{e x t}-H^{*}\right)^{2} / 2 \delta^{2}\right],
$$

where $H^{*}$ is the value of magnetic field, at which the flip of a cluster takes place. The expectation, $H^{*}$, and the standard deviation, $\delta$, in (3.3) are independent on $H_{\text {ext }}$ values. These parameters, as well as $H_{0} N$ product in Eq.(3.2) can be found by fitting the magnetization 


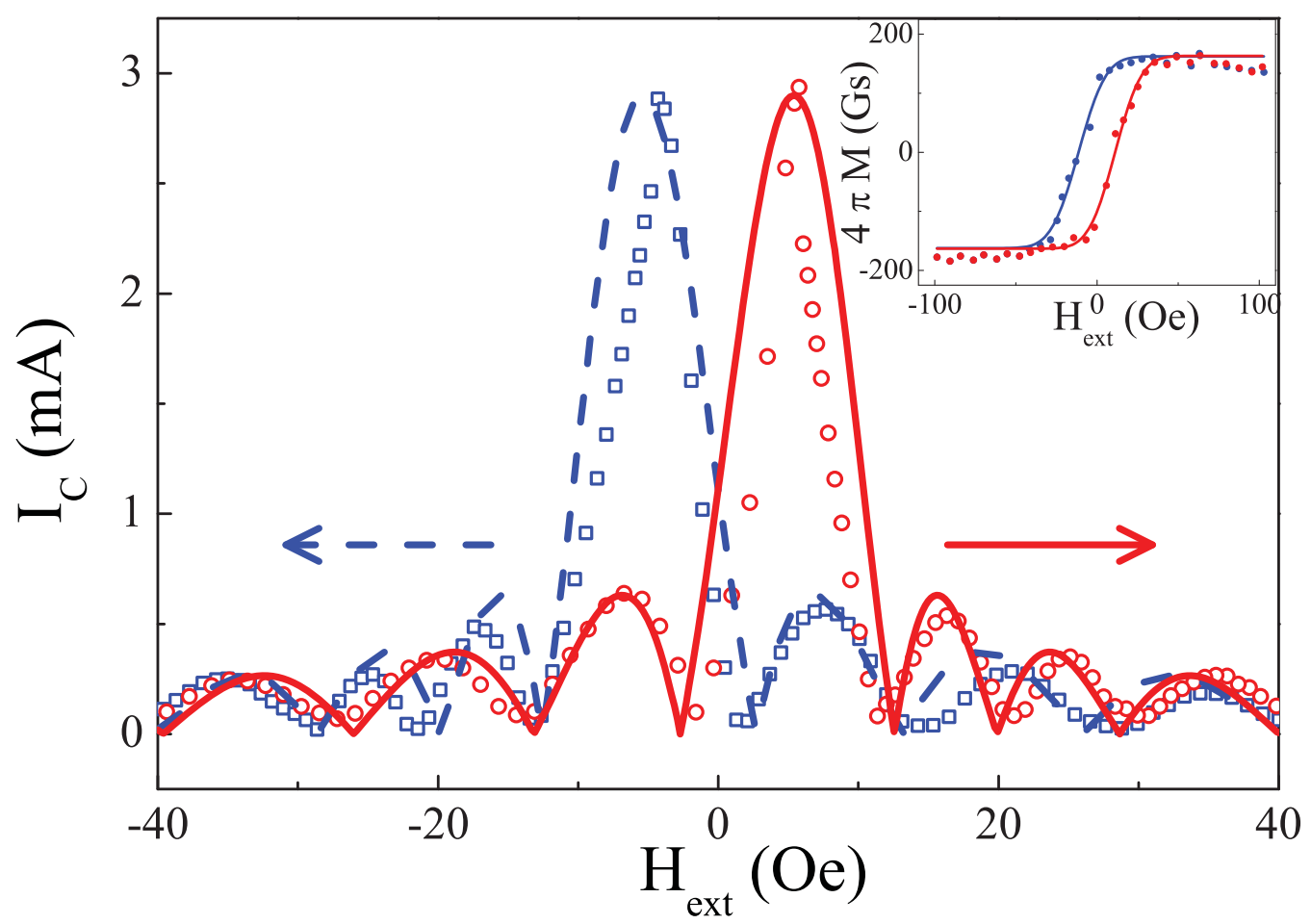

Figure 3.5. Dependence of critical current $I_{C}$ versus increasing (open circles) and decreasing (open squares) external magnetic field $H_{\text {ext }}$. Solid and dashed lines present the microscopic fitting of experimental data [16]. Inset shows the theoretical and experimental magnetization loops versus external magnetic field $H_{\text {ext }}$.

curve $4 \pi M\left(H_{\text {ext }}\right)$.

We apply this model to explain the data experimentally observed in Ref. [16] in SIsFS structures having cross-section area $10 * 10 \mu \mathrm{m}^{2}$ and F layer thickness $14 \mathrm{~nm}$. Figure 5 demonstrates the experimental dependencies of critical current $I_{C}$ versus increasing (open circles) and decreasing (open squares) external magnetic field $H_{\text {ext }}$. Solid and dashed lines present the microscopic fitting of the data. From hysteretic dependencies of F layer magnetization shown in insert in Fig. 3.5 we get $H^{*} \approx 11.4$ Oe, $\delta \approx 13$ Oe and $H_{0} N$ product $\approx 163$ Gs. The additional fitting parameters are $L_{\text {eff }} \approx 150 \mathrm{~nm}$ and the maximum value of the critical current.

The initial strong magnetic field $H_{\text {ext }}=-\infty$ remagnetize all clusters of $\mathrm{F}$ layer into the homogeneous state $n_{\downarrow}\left(H_{\text {ext }}\right)=1$. Gradual growth of the external field exhibits the conventional Fraunhofer pattern (solid line) with expected maximum at the positive value $H_{\text {ext }}$ corresponding to zero flux $\Phi=0$. However, the clusters start to flip around the point $H_{e x t}=H^{*}, n_{\uparrow}\left(H_{\text {ext }}\right)=\int_{-\infty}^{H_{e x t}} p\left(H_{\text {ext }}^{\prime}\right) d H_{\text {ext }}^{\prime}$. As a result, the rate of Fraunhofer oscillations increases. Similar situation takes place during field sweep- 
ing in the opposite direction (dashed line), from large positive to negative velues. The densities $n_{\uparrow}\left(H_{e x t}\right)$ and $n_{\downarrow}\left(H_{e x t}\right)=1-n_{\uparrow}\left(H_{e x t}\right)$ can be described by the expression, $n_{\uparrow}\left(H_{e x t}\right)=0.5\left(1+\operatorname{erf}\left(\left(H_{\text {ext }} \mp H^{*}\right) / \sqrt{2} \delta\right)\right)$, for forward and backward remagnitizations, respectively. Here $\operatorname{erf}(x)$ is the error function.

\subsection{Conclusion}

In this paper we have demonstrated a number of unique properties of SIsFS Josephson junctions. These structures exhibit large $I_{C} R_{N}$ product in the $\pi$ state comparable to that in SIS tunnel junctions commonly used in SFQ devices. Moreover, the whole structure behaves as a single junction with respect to an external magnetic field $H_{\text {ext }}$. Based on that, we have developed simple model describing the behavior of the critical current in these junctions in external field $H_{\text {ext }}$ taking into account remagnetization of the F-layer. The model explains asymmetric Fraunhofer oscillations $I_{C}\left(H_{e x t}\right)$ in $\mathrm{Nb}-\mathrm{Al} / \mathrm{AlO}_{x^{-}}$ $\mathrm{Nb}-\mathrm{Pd}_{0.99} \mathrm{Fe}_{0.01}-\mathrm{Nb}$ junctions reported in [15-17]. These effects provide the possibility to realize magnetic memory compatible with energy-efficient SFQ digital circuits [25] with high switching speed. 


\section{Bibliography}

[1] S. Oh, D. Youm, and M. Beasley, Appl. Phys. Lett. 71, 2376 (1997).

[2] L. R. Tagirov, Phys. Rev. Lett. 83, 2058 (1999).

[3] R. Held, J. Xu, A. Schmehl, C.W. Schneider, J. Mannhart, and M. Beasley, Appl. Phys. Lett. 89, 163509 (2006).

[4] V.V. Ryazanov, Physics - Uspekhi 42, 825 (1999).

[5] V.V. Ryazanov, V.A. Oboznov, A.Yu. Rusanov, A.V. Veretennikov, A.A. Golubov, J. Aarts, Phys. Rev. Lett. 86, 2427 (2001).

[6] F. S. Bergeret, A. F. Volkov, and K. B. Efetov, Phys. Rev. Lett. 86, 3140 (2001).

[7] A. A. Golubov, M. Yu. Kupriyanov, and Ya. V. Fominov, Pis'ma v ZhETF 75, 223 (2002) [JETP Letters 75, 190 (2002)].

[8] A. F. Volkov, F. S. Bergeret, and K. B. Efetov, Phys. Rev. B 64, 134506 (2001).

[9] M. Houzet and A. I. Buzdin, Phys. Rev. B 76, 060504(R) (2007).

[10] T. Yu. Karminskaya and M. Yu. Kupriyanov, Pis'ma v ZhETF 85, 343 (2007) [JETP Lett. 85, 286 (2007)].

[11] T. Yu. Karminskaya, M. Yu. Kupriyanov, and A. A. Golubov, Pis'ma v ZhETF 87, 657 (2008) [JETP Lett. 87, 570 (2008)].

[12] G. B. Halasz, M. G. Blamire, and J. W. A. Robinson, Phys. Rev. B 84, 024517 (2011).

[13] T. S. Khaire, M. A. Khasawneh, W. P. Pratt, Jr., and N. O. Birge, Phys. Rev. Lett. 104, $137002(2010)$.

[14] V. V. Bol'ginov, V. S. Stolyarov, D. S. Sobanin, A. L. Karpovich, and V. V. Ryazanov, Pis'ma v ZhETF 95, 408 (2012) [JETP Lett. 95, 366 (2012)].

[15] T. I. Larkin, V. V. Bol'ginov, V. S. Stolyarov, V. V. Ryazanov, I. V. Vernik, S. K. Tolpygo, and O. A. Mukhanov, Appl. Phys. Lett. 100, 222601 (2012). 
[16] I. V. Vernik, V. V. Bol'ginov, S. V. Bakurskiy, A. A.Golubov, M. Y. Kupriyanov, V. V. Ryazanov, O. A. Mukhanov, IEEE Trans. Appl. Supercond. 23, 1701208 (2013).

[17] V. V. Ryazanov, V. V. Bol'ginov, D. S. Sobanin, I. V. Vernik, S. K. Tolpygo, A. M. Kadin, O. A. Mukhanov, Physics Procedia 36, 35 (2012).

[18] L. S. Uspenskay, A. L. Rahmanov, L. A. Dorosinskiy, A. A. Chugunov, V. S. Stolyarov, O. V. Skryabina, and S. V. Egorov, Pis'ma v ZhETF 97, 176 (2013).

[19] K. D. Usadel, Phys. Rev. Lett. 25, 507 (1970).

[20] A. A. Golubov, M. Yu. Kupriyanov, E. Il’ichev, Rev. Mod. Phys. 76, 411 (2004).

[21] A. I. Buzdin, Rev. Mod. Phys. 77, 935 (2005).

[22] F. S. Bergeret, A. F. Volkov, K. B. Efetov, Rev. Mod. Phys. 77, 1321 (2005).

[23] M. Yu. Kuprianov and V. F. Lukichev, Zh. Eksp. Teor. Fiz. 94, 139 (1988) [Sov. Phys. JETP 67, 1163 (1988)].

[24] A. S. Vasenko, A. A. Golubov, M. Yu. Kupriyanov, and M. Weides, Phys. Rev. B 77, 134507 (2008).

[25] O.A. Mukhanov, IEEE Trans. Appl. Supercond. 21, 760 (2011). 


\section{Chapter 4}

\section{Anomalous surface states at interfaces in p-wave superconductors}

\subsection{Introduction}

Investigation of spin-triplet superconductivity is currently an exciting topic of research by the superconducting community. There are several experimental results in $\mathrm{Sr}_{2} \mathrm{RuO}_{4}[1-6]$ and in heavy fermion compounds [7-15] that are consistent with spin-triplet superconducting pairing. The promising paring symmetries are believed to be $p$-wave and $f$-wave in $\mathrm{Sr}_{2} \mathrm{RuO}_{4}[1-4,16]$ and $\mathrm{UPt}_{3}[17-19]$, respectively. Furthermore, to design $p$-wave superconductivity based on a proximity coupled system with a conventional $s$-wave superconductor and the semiconductor surface state of a topological insulator [20-29] has become a hot topic from the viewpoint of topological superconductivity [30-33]. The essential ingredients in these new systems are momentum-spin locking due to spin-orbit coupling and time reversal symmetry breaking by an external field.

In the above systems, it is known that surface Andreev bound state (SABS) [34-38] is generated inside the energy gap and stems from the topological properties of the bulk Hamiltonian [39]. The SABS has become a prominent concept since the debate over the pairing symmetry of high temperature superconductors (HTSs) [36, 40]. In HTSs, if the angle between the direction normal to the surface and the lobe direction of the $d$-wave pair potential deviates from zero, the injected quasiparticle and the reflected one can feel opposite signs of the pair potential depending on the injection angle [40]. The extreme case is that the above angle becomes $\pm \pi / 4$, where an injected quasiparticle always feels the sign change independent of the injection direction. This sign change of the pair potential produces SABS at zero energy and induces the zero bias conductance peak in tunneling spectroscopy $[37,40,41]$. The SABS has a flat dispersion along $k_{y}$, where $k_{y}$ is the momentum parallel to the surface. Actually, there are many experimental reports supporting ZBCP stemming from SABS [42-48].

When the zero energy SABS is located at the surface or interface, suppression of the pair potential in the main pairing channel occurs [49-52]. Furthermore, if the time 
reversal symmetry breaking is induced by the surface subdominant pair potential, ZBCP can split $[43,53-55]$. Thus, experimental study of the properties of ZBCP can serve as a guide to determine the symmetry of the pair potential and the possible presence of a subdominant one near the surface.

At the actual surface or interface, the diffusive scattering by the roughness due to atomic scale irregularity inevitably exists. It is known that surface roughness influences the electronic states of unconventional superconductors such as those of $d$-wave or $p$-wave type [56-58]. Studies of conductivity at the interfaces in $d$-wave superconductors have shown that their properties are strongly influenced by the degree of diffusive scattering of quasiparticles at the interface $[51,54,59-61]$. The higher the intensity of the diffusive scattering, the less pronounced the conductance peak at low voltages and the more pronounced the influence of subdominant components of order parameter on its shape.

Besides the above mentioned works, the theory of a proximity effect in diffusive normal metal / $d$-wave superconductor junctions has been developed [62]. It has been clarified that SABS can not penetrate into diffusive normal metal (DN) and the resulting ZBCP is broadened. These properties can be naturally explained using the concept of odd-frequency pairing [63]. The odd-frequency pairing states such as spin-singlet $p$-wave or spin-triplet $s$ wave can be generated by the translational symmetry breaking from the bulk conventional even-frequency pairing state, e.g., spin-singlet $s(d)$-wave or spin-triplet $p$-wave [64,65]. It is revealed that SABS in $d$-wave superconductor might be interpreted as an odd-frequency spin-singlet $p$-wave pairing $[64,66]$. However, $p$-wave pairing is fragile against diffusive scattering, so it can not penetrate into DN metal. This property is consistent with the fact that surface roughness has strong effect on ZBCP and SABS in $d$-wave superconductor.

On the other hand, a recent study of SBAS in $p$-wave superconductors has been stimulated by investigation of pairing symmetry in $\mathrm{Sr}_{2} \mathrm{RuO}_{4}$. The existing theory of the proximity effect in spin-triplet $p$-wave superconductors predicts that SABS produced by $p_{x^{-}}$ wave pairing can penetrate into DN metal attached to a spin-triplet $p_{x}$-wave superconductor [67]. This proximity effect induces many exotic phenomena including a zero enegy peak in the local density of state (LDoS) and negative local superfluid density [68-74]. Since the SBAS in a spin-triplet $p$-wave superconductor corresponds to odd-frequency spin-triplet $s$-wave pairing, it is robust against impurity scattering [66].

In actual $\mathrm{Sr}_{2} \mathrm{RuO}_{4}$, the promising symmetry is chiral $p$-wave pairing, i.e., $p_{x}+i p_{y}$ and one can expect more complex state as compared to $p_{x}$-wave or $d_{x y}$-wave cases. The resulting 
SABS has a linear dispersion as a function of $k_{y}[75,76]$ which is different from SABS in spin-singlet $d$-wave or spin-triplet $p_{x}$-wave superconductor. For a ballistic junction without any roughness, it has been shown that the resulting conductance exhibits a wide variety of line shapes including broad ZBCP or dip like structure around zero voltage [77-80]. Although it is not easy to obtain reliable tunneling spectroscopy data in the ab-plane junction experimentally, recent fabrication of well oriented junctions enabled detection of the SABS [81]. However, the effect of diffusive scattering has not been clarified yet. For a detailed comparison with experiment and predicted surface state, the research in this direction is needed. Since there are several relevant works in the surface state of superfluid ${ }^{3} \mathrm{He}[82,83]$, it is currently a challenging issue to study surface roughness effect on the surface density of states (SDoS) and pairing symmetry of chiral $p$-wave superconductors.

Despite the fact that previous studies revealed important aspects of these phenomena [84-86], there is still a need for systematic study and quantitative predictions. The purpose of this study is to evaluate the influence of the degree of diffusive electron scattering at interfaces in $p$-wave superconductors on the DoS.

The structure of this paper is the following: in Section 4.2 we formulate the problem and derive effective boundary conditions for diffusive surfaces in $p$-wave superconductors. In the following sections we discuss microscopic properties of pairing in such systems for the cases of both $p_{x}$ and chiral $p_{x}+i p_{y}$ symmetry. In the Sec.4.3 we focus on the spatial dependence of pair potential $\Delta$; Sec.4.4 is devoted to pair amplitudes $f$ and finally in Sec.4.5 we consider DoS for various surface properties.

\subsection{Model}

The description of the suppression of superconductivity in the main pairing channel and of the generation of subdominant order parameters can be done within the framework of the quasiclassical Eilenberger equations [87] within a two-dimensional model. To solve the problem, we will assume that the conditions of the clean limit are valid in the bulk superconductor region (scattering time $\tau \rightarrow \infty$ ) and the equations have the form

$$
\begin{gathered}
2 \omega f(x, \theta)+v \cos (\theta) \frac{d}{d x} f(x, \theta)=2 \Delta g(x, \theta), \\
2 \omega f^{+}(x, \theta)-v \cos (\theta) \frac{d}{d x} f^{+}(x, \theta)=2 \Delta^{*} g(x, \theta),
\end{gathered}
$$




$$
2 v \cos (\theta) \frac{d}{d x} g_{\omega}(x, \theta)=2\left(\Delta^{*} f_{\omega}-\Delta f_{\omega}^{+}\right) .
$$

Here $g(x, \theta), f(x, \theta)$ and $f^{+}(x, \theta)$ are normal and anomalous Eilenberger functions, $\Delta(x)$ is pair potential, $\theta$ is angle between the vector normal to the interface and the direction of the electron Fermi velocity $v ; \omega=\pi T(2 n+1)$ are Matsubara frequencies and $T$ is temperature, $x$ is coordinate along the axis normal to the boundary. The form of selfconsistency equation is sensitive to the chosen symmetry of pair potential. In the case of $p_{x}$-wave pairing potential $\Delta=\Delta_{x} \cos (\theta)$ leads to equation

$$
\Delta_{x} \ln \frac{T}{T_{c}}+2 \pi T \sum_{\omega} \frac{\Delta_{x}}{\omega}-\left\langle 2 \cos \left(\theta^{\prime}\right) f\left(x, \theta^{\prime}\right)\right\rangle=0 .
$$

The other type of chiral $p_{x}+i p_{y}$ symmetry relates to $\Delta=\Delta_{x} \cos (\theta)+i \Delta_{y} \sin (\theta)$ with similar self-consistent equation [88]

$$
\begin{aligned}
& \Delta_{x} \ln \frac{T}{T_{c}}+2 \pi T \sum_{\omega} \frac{\Delta_{x}}{\omega}-\left\langle 2 \cos \left(\theta^{\prime}\right) \operatorname{Re} f\left(x, \theta^{\prime}\right)\right\rangle=0 \\
& \Delta_{y} \ln \frac{T}{T_{c}}+2 \pi T \sum_{\omega} \frac{\Delta_{y}}{\omega}-\left\langle 2 \sin \left(\theta^{\prime}\right) \operatorname{Im} f\left(x, \theta^{\prime}\right)\right\rangle=0 .
\end{aligned}
$$

Here $\langle\ldots\rangle=(1 / 2 \pi) \int_{0}^{2 \pi}(\ldots) d \theta$ and $T_{c}$ is the critical temperature. Note that the considered case of chiral p-wave supercondutor is equivalent to a thin film of superfluid $3 \mathrm{He}$ A-phase. The polar phase of 3 He has been recently identified in aerogel [89,90].

Diffusive properties of the interface will be described in the Ovchinnikov model [91] , i.e. it is simulated by a thin diffusive layer of thickness, $d \ll \xi_{\text {eff }}=\min \left\{\sqrt{\xi_{0} l_{e}}, \xi_{0}\right\}$, $\xi_{0}=v / 2 \pi T_{c}$, with strong electron scattering inside. Here $l_{e}$ is electron mean free path and $\tau=l_{e} / v$. Inside this layer, located in the area $0 \leq x \leq d$, we can neglect terms in the Eilenberger equations [87] that are proportional to $\omega$ and $\Delta$

$$
\begin{aligned}
v \cos (\theta) \frac{d}{d x} f(x, \theta) & =\frac{1}{\tau}(g\langle f\rangle-f\langle g\rangle), \\
v \cos (\theta) \frac{d}{d x} f^{+}(x, \theta) & =\frac{1}{\tau}\left(g\left\langle f^{+}\right\rangle-f^{+}\langle g\rangle\right), \\
2 v \cos (\theta) \frac{d}{d x} g(x, \theta) & =\frac{1}{\tau}\left(f\left\langle f^{+}\right\rangle-f^{+}\langle f\rangle\right)
\end{aligned}
$$

and assume that $\langle f\rangle,\left\langle f^{+}\right\rangle$and $\langle g\rangle$ are spatially independent quantities, which should be determined selfconsistently during the process of finding solutions of the system (4.1)-(4.9). 


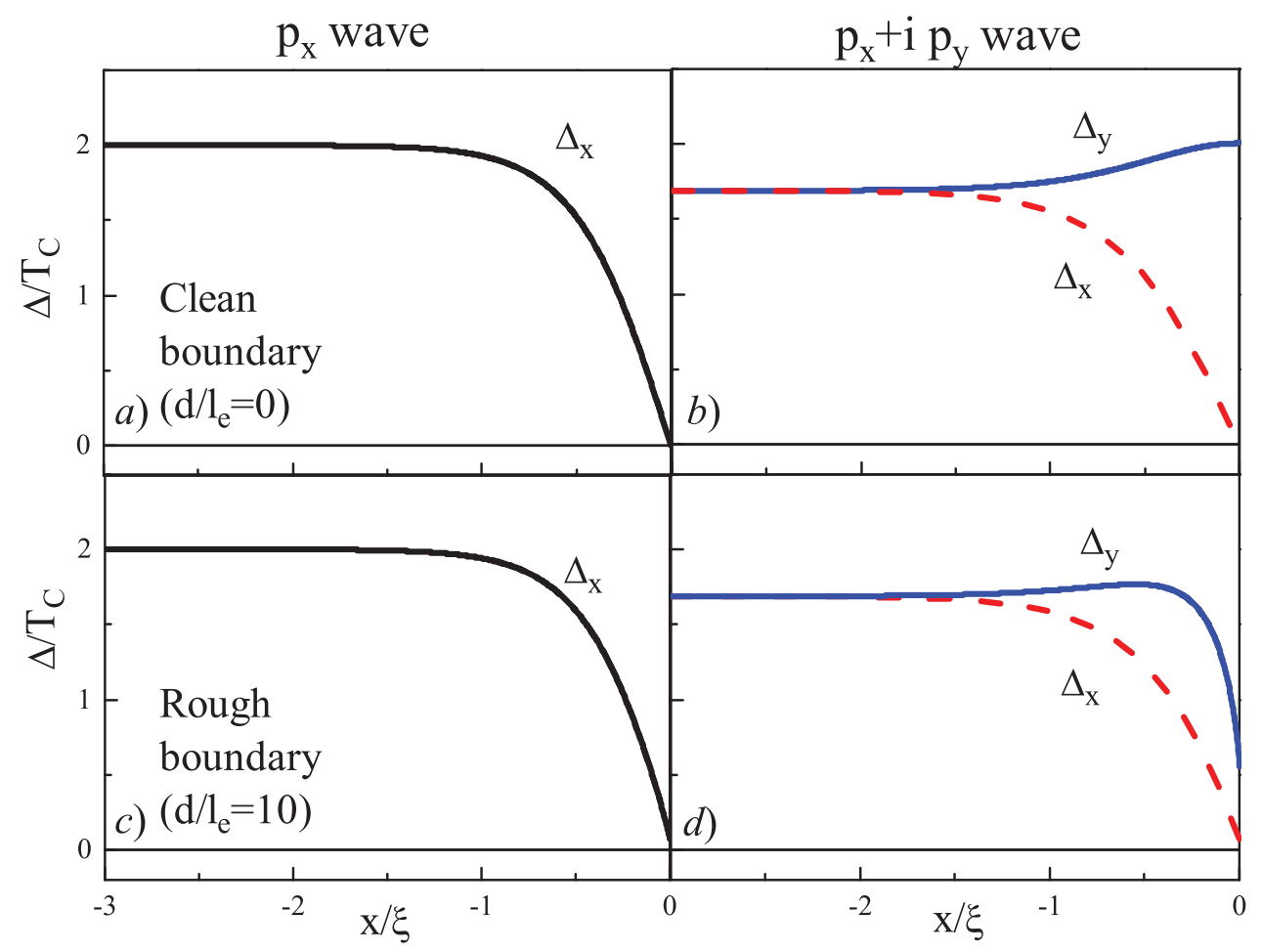

Figure 4.1. Pair potential $\Delta$ as a function od coordinate $x$ in the vicinity of surface for a) $p_{x}$-wave superconductor with clean surface, b) $p_{x}+i p_{y}$-wave superconductor with clean surface, a) $p_{x}$-wave superconductor with rough surface, a) $p_{x}+i p_{y}$-wave superconductor with rough surface. On the panels b) and d) solid and dashed lines correspond to components $\Delta_{y}$ and $\Delta_{x}$ respectively. Tha parameter $d / l_{e}$ determines the degree of the surface roughness.

For the development of numerical algorithms for solving the Eilenberger equations it is convenient to rewrite them using the Ricatti parametrization [64,92].

$$
f_{ \pm}=\frac{2 a_{ \pm}}{1+a_{ \pm} b_{ \pm}}, f_{ \pm}^{+}=\frac{2 b_{ \pm}}{1+a_{ \pm} b_{ \pm}}, g_{ \pm}=\frac{1-a_{ \pm} b_{ \pm}}{1+a_{ \pm} b_{ \pm}}
$$

that are defined in the angle $-\pi / 2 \leq \theta \leq \pi / 2$. Their substitution into (4.1)-(4.9) leads to the general relations in the form

$$
\begin{aligned}
& v \cos (\theta) \frac{d}{d x} a_{ \pm}=\Delta\left[1-a_{ \pm}^{2}\right] \mp 2 \omega a_{ \pm} \\
& v \cos (\theta) \frac{d}{d x} b_{ \pm}=-\Delta\left[1-b_{ \pm}^{2}\right] \pm 2 \omega b_{ \pm}
\end{aligned}
$$


in the clean superconducting region and

$$
\begin{aligned}
& v \cos (\theta) \frac{d}{d x} a_{ \pm}= \pm \frac{1}{2 \tau}\left[\langle f\rangle-a_{ \pm}^{2}\left\langle f^{+}\right\rangle-2 a_{ \pm}\langle g\rangle\right] \\
& v \cos (\theta) \frac{d}{d x} b_{ \pm}=\mp \frac{1}{2 \tau}\left[\left\langle f^{+}\right\rangle-b_{ \pm}^{2}\langle f\rangle-2 b_{ \pm}\langle g\rangle\right]
\end{aligned}
$$

in the diffusive layer. The subscript \pm indicates the direction of motion along the trajectory towards the boundary $(+)$ or away from it $(-)$. For $x \rightarrow-\infty$ we have

$$
\begin{aligned}
& a_{ \pm}= \pm \frac{\Delta}{\omega+\sqrt{\omega^{2}+|\Delta|^{2}}} \\
& b_{ \pm}= \pm \frac{\Delta^{*}}{\omega+\sqrt{\omega^{2}+|\Delta|^{2}}}
\end{aligned}
$$

where $\Delta$ is the bulk value of pair potential.

Finally, the problem must be supplemented by boundary conditions at the free surface of the diffusion layer $(x=d)$

$$
\begin{aligned}
& b_{+}(d,-\theta)=b_{-}(d, \theta), \\
& a_{-}(d,-\theta)=a_{+}(d, \theta) .
\end{aligned}
$$

The boundary conditions (4.17), (4.18) differ significantly from those used previously [61]

$$
b_{+}(d,-\theta)=a_{+}(d, \theta)
$$

in the analysis of the influence of diffuse scattering on the superconducting correlations in $d$-wave superconductors. Indeed, in the $d$-wave case the following relations

$$
b_{ \pm}(x, \theta)=a_{\mp}(x, \theta)
$$

hold, and then the conditions (4.17), (4.18) are reduced to the relation (4.19). As a result, further analysis in the $d$-wave case was based not on four, but only on two Eilenberger functions. It should be also pointed out that when writing conditions (4.17-4.18) we essentially used not only the fact that the particle reflected from the free surface must diffuse into the node with a opposite value of the order parameter, but also the fact that its velocity should be directed into the interior of superconductor. That is why in the right side of (4.17) there is a function $b_{-}(d, \theta)$, and there is not $a_{+}(d, \theta)$, or some combination of them.

The boundary value problem (4.11)-(4.18) has been solved analytically (see Ap- 


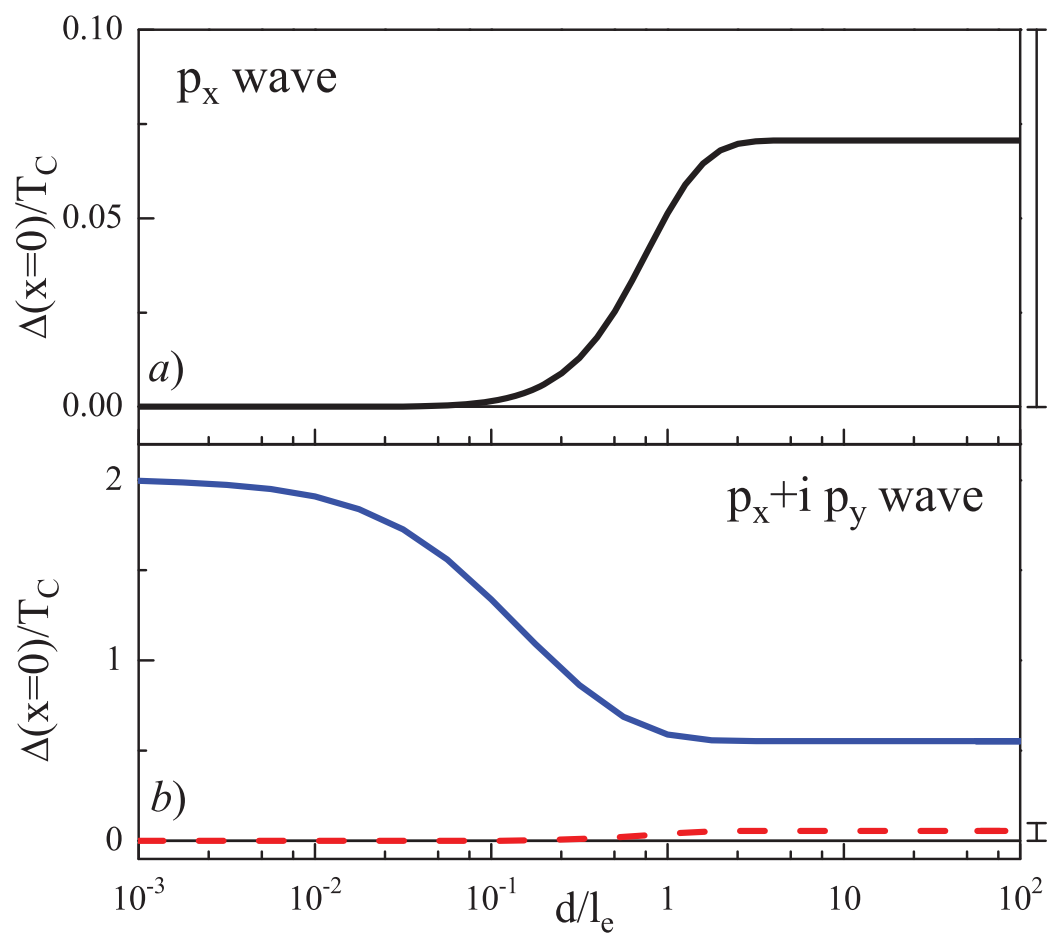

Figure 4.2. Pair potential $\Delta$ on a surface of diffusive layer versus its roughness $d / l_{e}$ a) for $p_{x}$-wave superconductor and b) $p_{x}+i p_{y}$-wave superconductor. Solid and dashed lines on the panel b) correspond to components $\Delta_{y}$ and $\Delta_{x}$ respectively.

pendix) resulting in an effective boundary condition at the interface between the clean $p$-wave area and the diffusive layer at the $x=0$. It is expressed as the relation between the functions of a coming into the diffusive layer $a_{+}(0,-\theta), b_{-}(0,-\theta)$ and leaving out from it $a_{-}(0, \theta), b_{+}(0, \theta)$.

$$
\begin{aligned}
& a_{-}(0, \theta)=\frac{a_{+}(0,-\theta)-\left(G a_{+}(0,-\theta)-F\right) \tanh \{k d\}}{\left(F^{+} a_{+}(0,-\theta)+G\right) \tanh \{k d\}+1}, \\
& b_{+}(0, \theta)=\frac{b_{-}(0,-\theta)-\left(G b_{-}(0,-\theta)-F^{+}\right) \tanh \{k d\}}{\left(F b_{-}(0,-\theta)+G\right) \tanh \{k d\}+1} .
\end{aligned}
$$

Here $k$ is an effective wave vector in dirty layer

$$
k=\frac{\sqrt{\left\langle g_{+}+g_{-}\right\rangle^{2}+\left\langle f_{+}^{+}+f_{-}^{+}\right\rangle\left\langle f_{+}+f_{-}\right\rangle}}{\ell \cos (\theta)},
$$


and $F, F^{+}$and $G$ are parametrized averages of Green functions

$$
\begin{aligned}
F & =\frac{\left\langle f_{+}+f_{-}\right\rangle}{\sqrt{\left\langle g_{+}+g_{-}\right\rangle^{2}+\left\langle f_{+}^{+}+f_{-}^{+}\right\rangle\left\langle f_{+}+f_{-}\right\rangle}}, \\
F^{+} & =\frac{\left\langle f_{+}^{+}+f_{-}^{+}\right\rangle}{\sqrt{\left\langle g_{+}+g_{-}\right\rangle^{2}+\left\langle f_{+}^{+}+f_{-}^{+}\right\rangle\left\langle f_{+}+f_{-}\right\rangle}}, \\
G & =\frac{\left\langle g_{+}+g_{-}\right\rangle}{\sqrt{\left\langle g_{+}+g_{-}\right\rangle^{2}+\left\langle f_{+}^{+}+f_{-}^{+}\right\rangle\left\langle f_{+}+f_{-}\right\rangle}} .
\end{aligned}
$$

Here the averaging operation is performed over the range of angles, $-\pi / 2 \leq \theta \leq \pi / 2$, that is $\langle\ldots\rangle=(1 / 2 \pi) \int_{-\pi / 2}^{\pi / 2}(\ldots) d \theta$.

The above boundary conditions are the main analytical result of this paper and they provide the framework for a quantitative selfconsistent study of surface effects in $p$-wave superconductors. The results of this study are presented below.

\subsection{Pair potential $\Delta$}

To study properties of the systems we have developed the method of numerical solution of the boundary-value problem (4.11)-(4.18). According to this method, outside of the diffusive layer (in the region where $(\tau \rightarrow \infty)$ ), the equations (4.11), (4.12) for $a_{+}(x, \theta)$ and $b_{-}(x,-\theta)$ are numerically integrated starting from conditions (4.15) at infinity $(x=-\infty)$ and moving along the trajectory towards the boundary $(x=0)$. As a result, functions $a_{+}(0, \theta)$ and $b_{-}(0,-\theta)$ in equations $(4.21),(4.22)$ are calculated. Then, starting values $a_{-}(0, \theta)$ and $b_{+}(0, \theta)$ are determined from the boundary conditions (4.21), (4.22), and functions $a_{-}(x, \theta)$ and $b_{+}(x, \theta)$ are obtained by integration along the trajectories going out of the diffusive layer. The coefficients $F, F^{+}$and $G$ in (4.21), (4.22) and the spatial dependence of the order parameter $\Delta(x)$ are determined in an iterative self-consistent way using Eqs. (4.21)-(4.26) and (4.4)-(4.6), respectively. All the calculations below were performed at temperature $T=0.5 T_{C}$.

According to this procedure, we calculate spatial distributions of pair potential $\Delta(x)$, pair amplitude $f(x)$ and surface DoS (SDoS) for different thicknesses of diffusive layer.

Figure 4.1 shows spatial dependencies of the pair potential $\Delta(x)$ for $p_{x}$ and chiral $p_{x}+i p_{y}$ cases. In a $p_{x}$-wave superconductor, the amplitude of the pair potential $\Delta_{x}$ reaches its maximum value in the bulk $\left(\Delta_{x} \approx 2 T_{C}\right.$ at $\left.T=0.5 T_{C}\right)$. In the vicinity of the interface it 

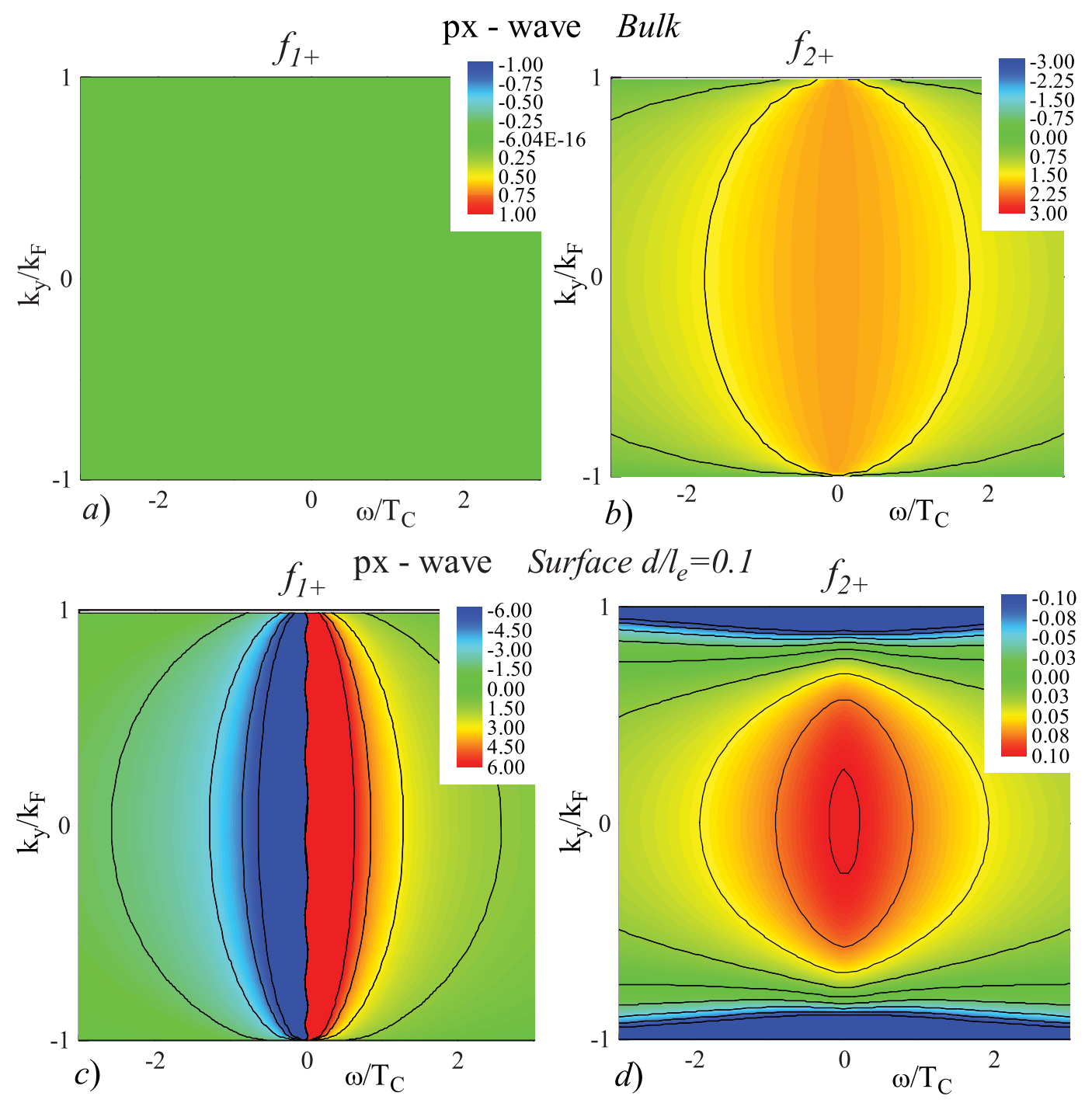

Figure 4.3. (Color Online) Odd and even angle resolved pairing amplitudes $f_{1}$ and $f_{2}$ as functions of $k_{y}$ and $\omega \mathrm{a}$ ), b) in the bulk $p_{x}$-wave superconductor and c),d) at the surface $(x=0)$ with roughness $d / l_{e}=0.1$

is suppressed up to zero in the absence of a diffusive layer. It comes from the fact that the reflection of electrons takes place into the band with negative sign of pair potential (See Fig. 4.1a). The presence of roughness does not change the general shape of the dependence and only provides slight growth of the pair potential $\Delta_{x}(0)$ at the surface (Figs. 4.1c and $4.2 \mathrm{a})$.

For the case of chiral symmetry, the impact of surface is more diverse (See Fig. $4.1 \mathrm{~b}, \mathrm{~d})$. In contrast to the former case, the bulk pair potential has the BCS magnitude (for the considered temperature $\Delta_{x}=\Delta_{y} \approx 1.67 T_{C}$ ) due to spherical symmetry of $|\Delta|$. As in the previous case, the component $\Delta_{x}$ is suppressed in the vicinity of a surface. In 


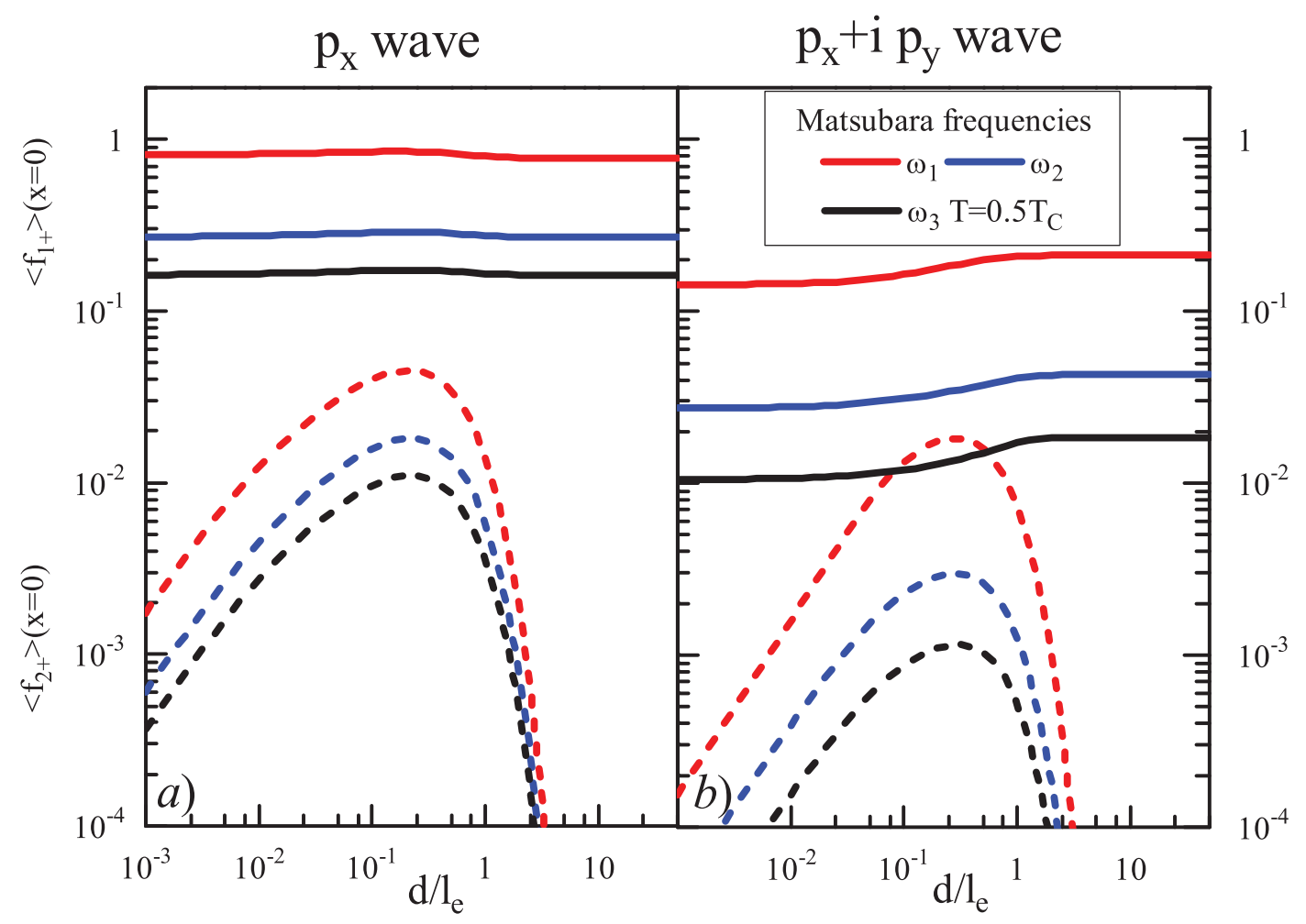

Figure 4.4. Odd $<f_{1}>$ and even $<f_{2}>$ components of pair amplitude at a surface $(x=0)$ versus its roughness $d / l_{e}$. Panels a) and b) correspond to $p_{x}$-wave and to $p_{x}+i p_{y}$-wave respectively. Pair amplitudes are angle averaged and calculated for the first, the second and third Matsubara frequencies $\omega_{n}$ at temperature $T=0.5 T_{C}$.

contrary, the component $\Delta_{y}$ grows up to the bulk value for $p_{y}$ symmetry in the case of a clean surface. However, $\Delta_{y}$ is sensitive to a degree of surface roughness: the pair potential component $\Delta_{y}$ decreases by about three times in comparison with bulk value in the limit of large roughness (Fig. 4.2b). This property has a simple qualitative explanation: in the clean limit the incident and reflected electrons fill the same sign of pair potential, while in the diffusive case some of the reflected electrons fills the opposite sign of the pair potential due to impurity scattering. In the following we will see that this phenomenon manifests itself in the DoS at a surface.

\subsection{Pair amplitudes $f$ and $f^{+}$}

An important characteristic of the considered system is a relation between surface roughness and the time-parity of the pairing amplitude $f$ near the surface. Let us introduce 


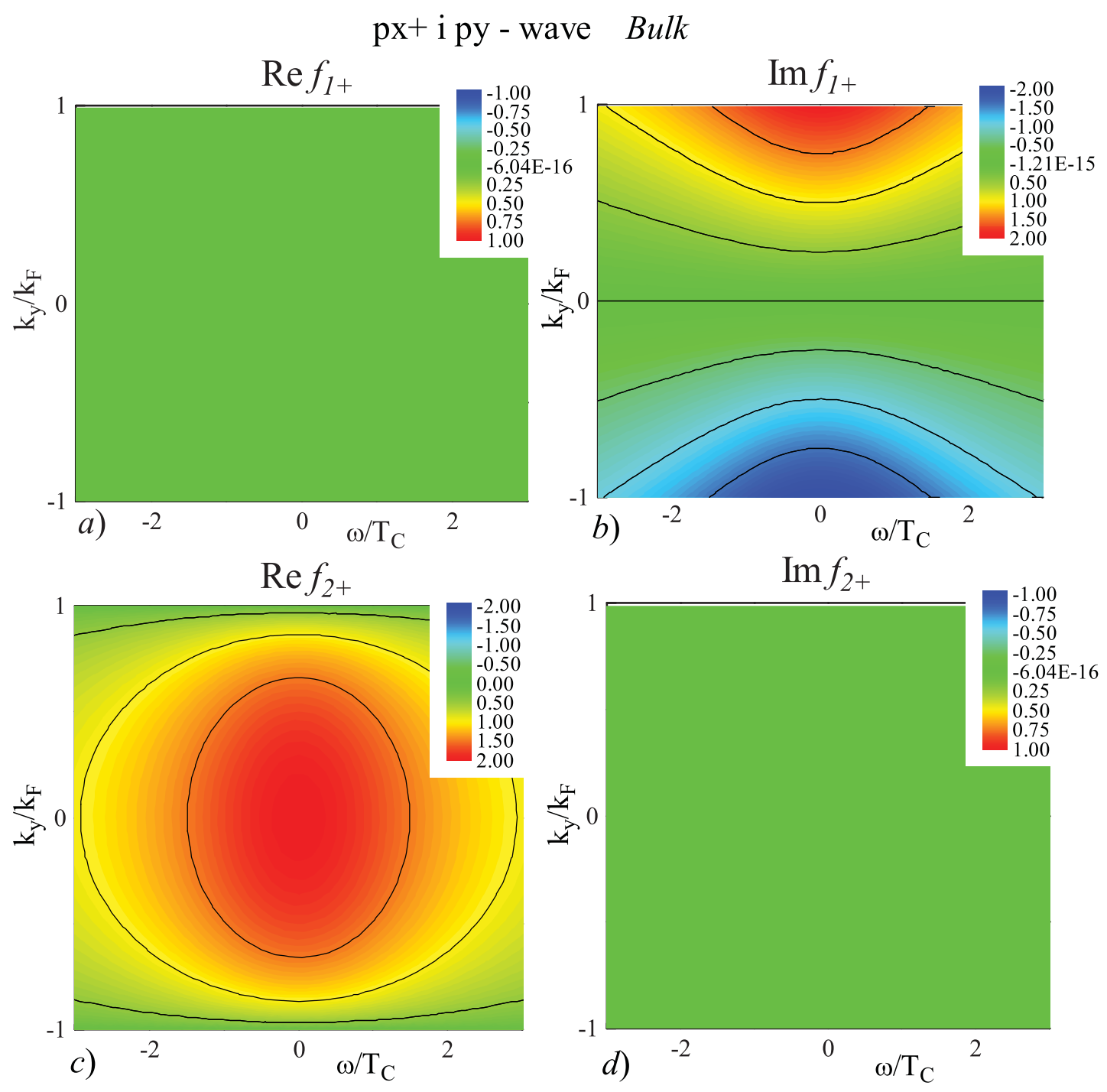

Figure 4.5. (Color Online) Odd and even angle resolved pairing amplitudes $f_{1}$ and $f_{2}$ in the bulk chiral superconductor as functions of $k_{y}$ and $\omega$.

the symmetrized functions

$$
\begin{aligned}
& f_{1 \pm}=f_{ \pm}-f_{ \pm}^{+}, \\
& f_{2 \pm}=f_{ \pm}+f_{ \pm}^{+} .
\end{aligned}
$$

As follows from Eqs. (4.1)-(4.3) (see Appendix B), these Green functions have the following symmetries with respect to the Matsubara frequency:

$$
g_{\omega}=-g_{-\omega}^{*} ; \quad f_{1, \omega}=-f_{1,-\omega}^{*} ; \quad f_{2, \omega}=f_{2,-\omega}^{*}
$$

and with respect to the angle of motion $\theta$ 

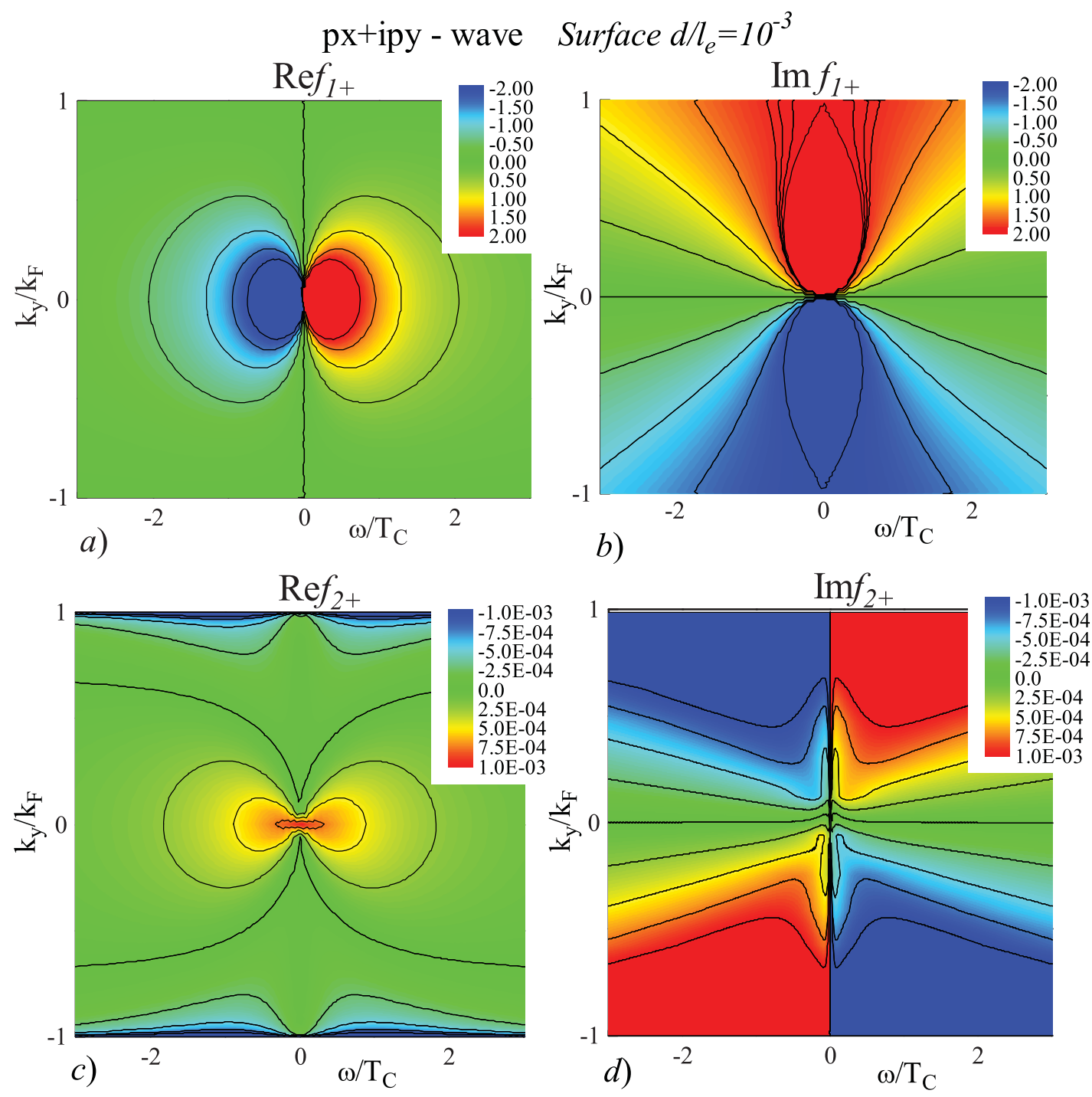

Figure 4.6. (Color Online) Odd and even angle resolved pairing amplitudes $f_{1}$ and $f_{2}$ as functions of $k_{y}$ and $\omega$ at the almost mirror surface $(x=0)$ with roughness $\left(d / l_{e}=0.001\right)$ for the chiral superconductor.

$$
f_{1}(\theta)=f_{1}^{*}(-\theta) ; \quad f_{2}(\theta)=f_{2}^{*}(-\theta) ; \quad g(\theta)=g^{*}(-\theta) .
$$

Such symmetry also means that imaginary parts of these functions are antisymmetric over $\theta$ and disappear after averaging over $\theta$. Therefore, the average quantities $\left\langle f_{1,2}>\right.$ are real functions and we can call function $<f_{1}>$ odd-frequency and $<f_{2}>$ even-frequency. To demonstrate this property, we trace the behavior of $\left\langle f_{1}>\right.$ and $<f_{2}>$ in detail in the bulk superconductor and at the surface.

For a $p_{x}$-wave superconductor, the problem can be simplified and can be solved in terms of real values: the function $f_{1}\left(f_{2}\right)$ is symmetric over angle $\theta$ and odd (even) 

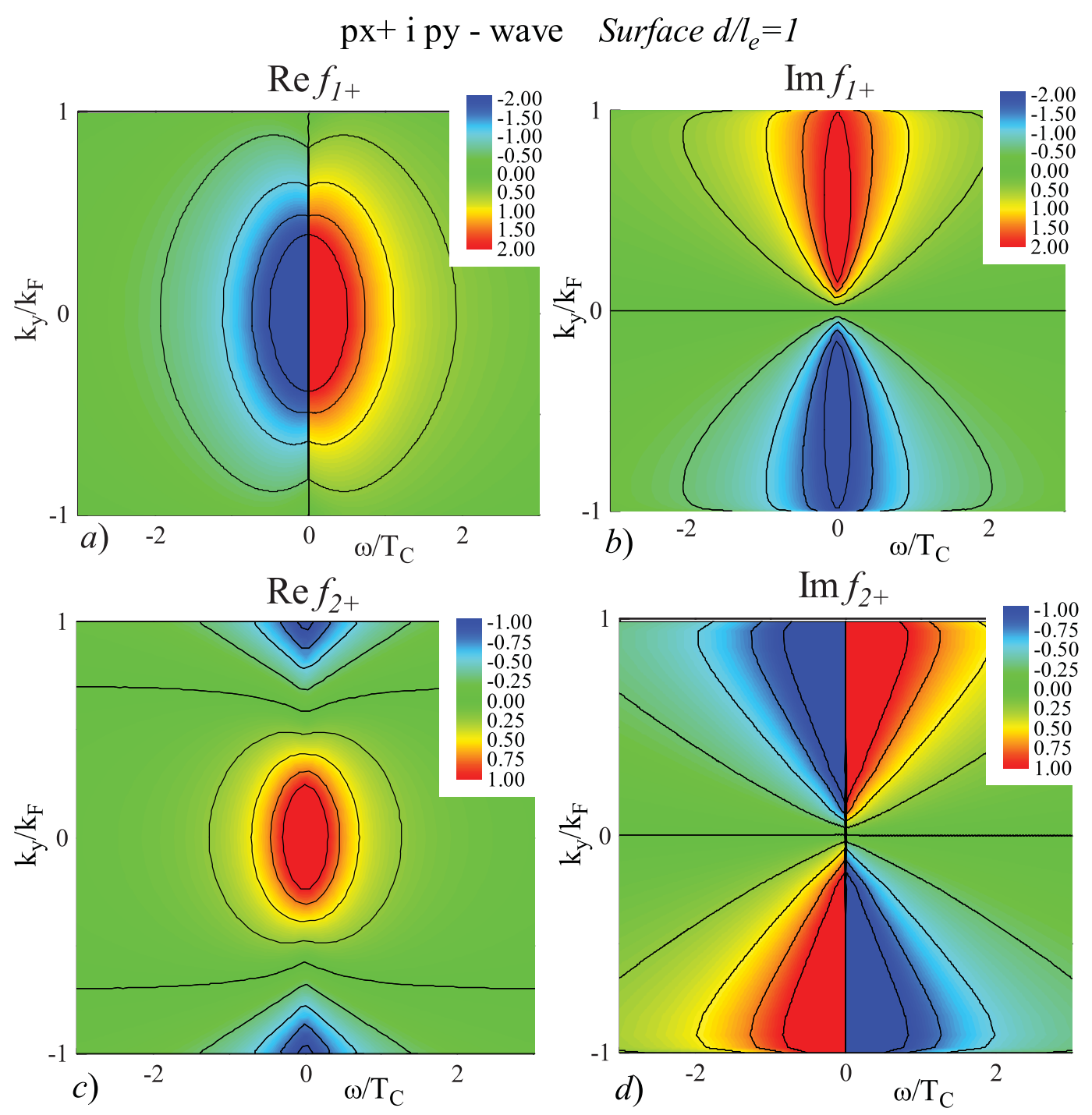

Figure 4.7. (Color Online) Odd and even angle resolved pairing amplitudes $f_{1}$ and $f_{2}$ as functions of $k_{y}$ and $\omega$ at the surface $(x=0)$ with roughness $\left(d / l_{e}=1\right)$ for the chiral superconductor.

over frequency $\omega$. We will focus on the functions $f_{1+}$ and $f_{2+}$ corresponding to incident trajectories. In the bulk superconductor only the even-frequency component $f_{2+}$ exists in full accordance with analytical solutions (4.15)-(4.16) (Fig. 4.3a,b). Hereinafter, we will present angle dependencies in terms of parallel component of the Fermi wave-vector $k_{y}=k_{F} \sin (\theta)$. At the surface the formation of another component takes place: electrons reflect into the lobe with different sign of order parameter (in accordance with Eqs. (4.17)(4.18)) and an odd-frequency Green function $f_{1+}$ (Fig. 4.3c) is generated. Its amplitude diverges in the limit $\omega \rightarrow 0$, but remains finite at a certain Matsubara frequency $\omega_{n}$.

The behavior of even-frequency $f_{2+}$ is a quite complex. At the mirror surface it 
is fully destroyed by direct reflection of particles in accordance with Eqs. (4.17)-(4.18). Surface roughness leads to generation of even-frequency Green function $f_{2+}$ since reflected amplitudes $a_{-}$and $b_{+}$become isotropic. However the average value $\left\langle f_{1+}\right\rangle$ during further isotropization reaches its maximum and starts to decrease for larger roughness values (See Fig. 4.4a). This effect occurs because $f_{2+}$ has different signs at angles $\theta$ in the vicinity of $\pm \pi / 2$ and $\theta=0$ and in the limit of a thick diffusive layer these angle areas compensate each other during integration.

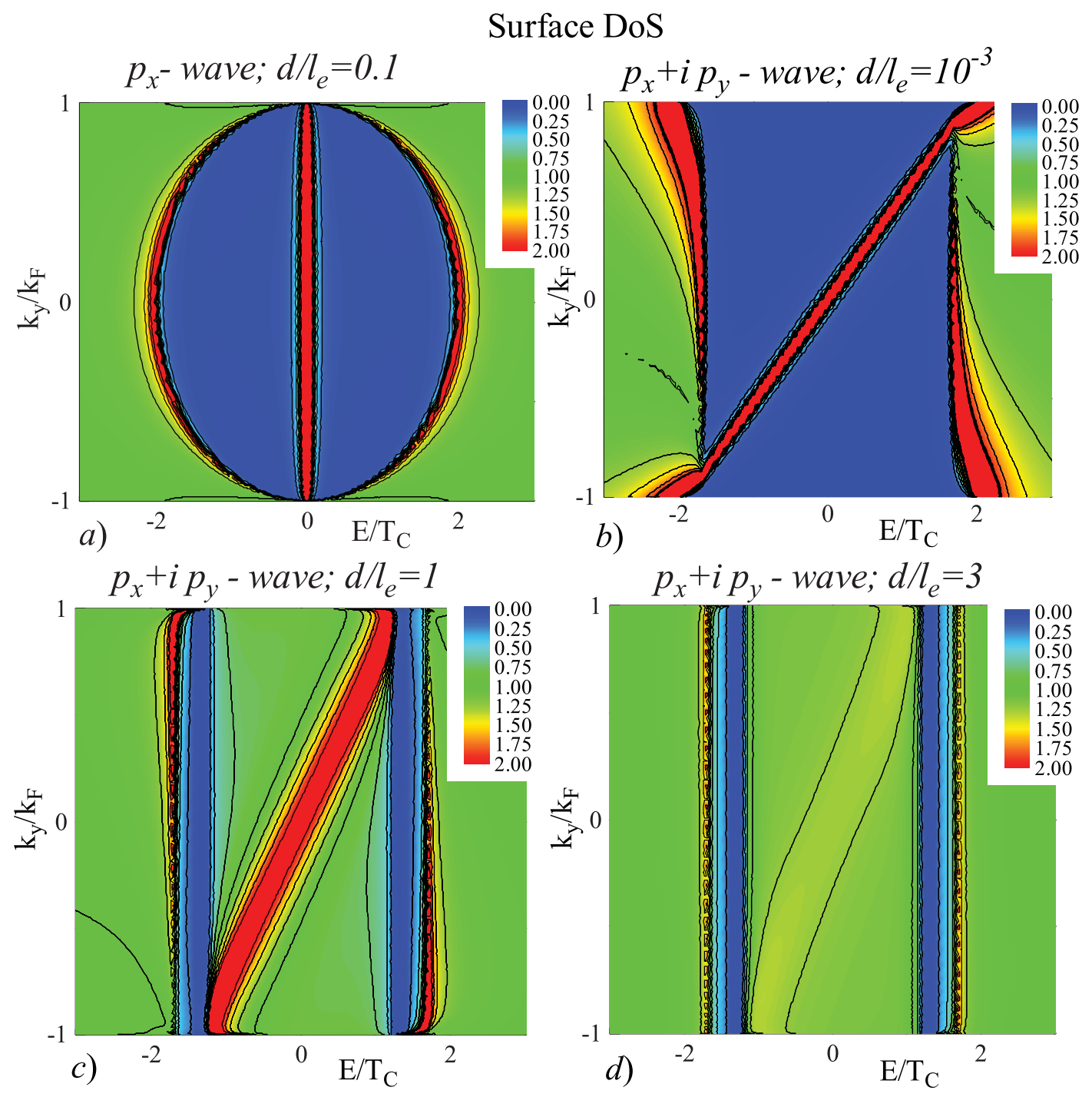

Figure 4.8. (Color Online) Surface $x=d$ angle-resolved DoS for a) $p_{x}$-wave with surface roughness $d / l_{e}=0.1$ and for b), c), d) $p_{x}+i p_{y}$-wave with roughness $d / l_{e}=10^{-3}, d / l_{e}=1$, $d / l_{e}=3$ respectively.

In the chiral $p_{x}+i p_{y}$-wave superconductors the general properties of the Green functions are pretty similar: in the bulk all odd-frequency components of Green functions 
Re $f_{1}$ and $\operatorname{Im} f_{2}$ don't exist (Fig. 4.5a,d) and even ones correspond to the symmetry of real and imaginary parts of pair potential $\Delta$. Thus $\operatorname{Re} f_{2+}$ has maximum at $\theta=0$, in accordance with the angle-dependence of $p_{x}$-component, and $\operatorname{Im} f_{1+}$ reaches its maximum values at $\theta=\pi / 2$ in accordance with $i p_{y}$-one. (Fig. $4.5 \mathrm{~b}, \mathrm{c}$ )

In the vicinity of the surface other components also arise. Particles reflected from the mirror boundary into the $p_{x}$-band with different sign of order parameter generate an oddfrequency pair amplitude. However, in imaginary values the sign of the $p_{y}$-component of the order parameter is conserved after reflection and hold even-frequency symmetry. Thus in this case there are only two significant components of Green functions: odd-frequency Re $f_{1+}$ with maximum at $(\theta=0)$ and even-frequency $\operatorname{Im} f_{1+}$ increasing for large angles. (See Fig. 4.6). In the structures with finite thickness of diffusive layer another Green function components arise. Isotropization of $a_{-}$and $b_{+}$leads to the formation of nonzero components Re $f_{2}$ and $\operatorname{Im} f_{2}$. At greater roughness they increase further (Fig. 4.7), but the averaged value of $\left\langle f_{2+}\right\rangle$ falls down due to negative contribution from large angles.

To show this clearly we present angle averaged pair amplitudes $\left|\left\langle f_{1+}\right\rangle\right|$ and $\left|\left\langle f_{2+}\right\rangle\right|$ at the surface versus roughness (Fig. 4.4) for the first, the second and the third Matsubara frequencies at fixed temperature $T=0.5 T_{C}$. Odd-frequency amplitude $\left|\left\langle f_{1+}\right\rangle\right|$ significantly exceeds even-frequency one $\left|\left\langle f_{2+}\right\rangle\right|$ in cases of both $p_{x}$ and chiral $p_{x}+i p_{y}$ symmetries. Furthermore, in limits of both low and high roughness the even-frequency component $\left|\left\langle f_{2+}\right\rangle\right|$ vanishes. At the same time, we have found that this component reaches its maximum value in the finite roughness range. This means that new effects exist in the range of intermediate roughness and one may expect a qualitative difference in measurable properties such as DoS in this regime.

\subsection{Density of States}

To calculate DoS, one can solve the same system of equations (4.11)-(4.18), where Matsubara frequency is replaced by energy $\omega \rightarrow i E$. Further we will focus only on DoS for incident electrons because it is this quantity which is probed in tunnel experiments

$$
D o S=R e\left(g_{+}\right)=\operatorname{Re}\left(\frac{1+a_{+} b_{+}}{1-a_{+} b_{+}}\right)
$$

Odd-frequency pairing around the surface leads to formation of subgap bound states, which occur as peaks in the angle resolved density of states (ARDoS) in both $p_{x}$ and $p_{x}+i p_{y}$ 


\section{Distribution of ARDoS}

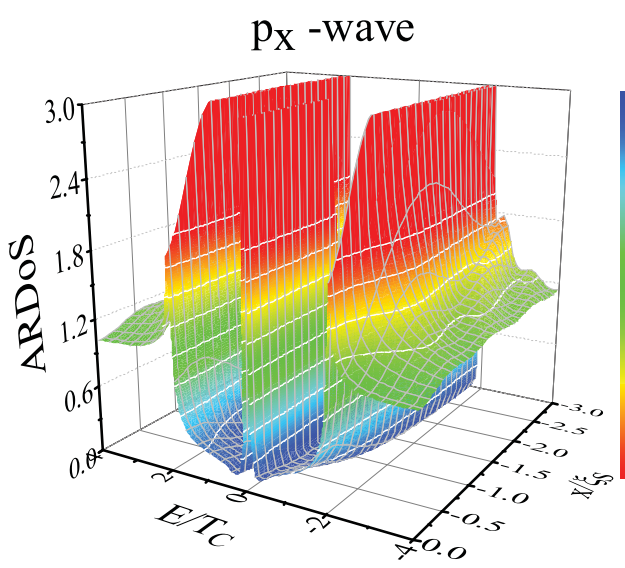

a)

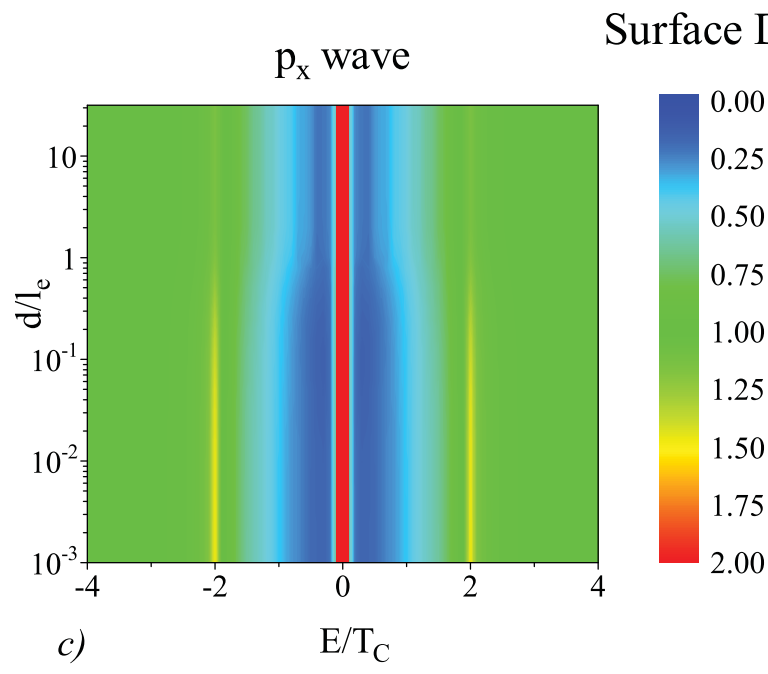

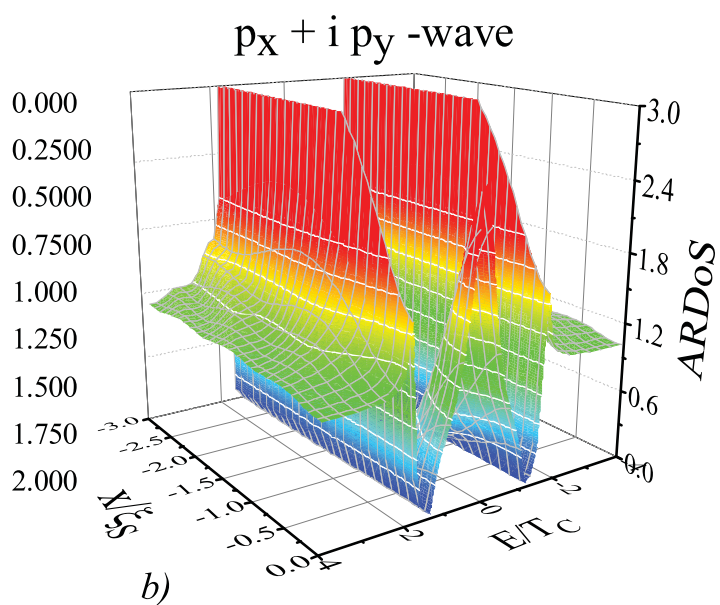

b)

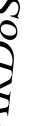

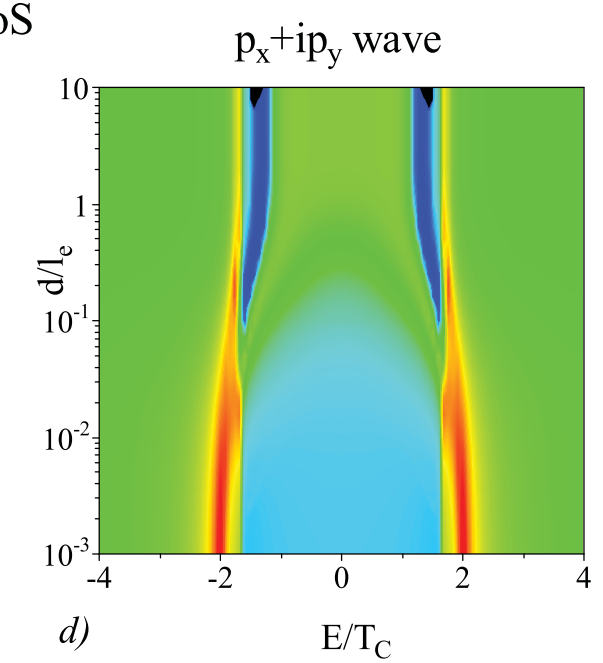

Figure 4.9. (Color Online) a,b) Spatial distribution of $\operatorname{ARDoS}(\theta=0)$ for the $p_{x}$-wave and $p_{x}+i p_{y}$-wave superconductors. c-d) Angle Averaged SDoS as a function of surface roughness.

cases. Figure 4.8 shows ARDoS at the surface for $p_{x}$-wave (a) and for chiral superconductor (b, c, d) and reveals the behavior of the subgap bound states as a function of angle of propagation $\sin (\theta)$ and momentum $k_{y}$, respectively. In the $p_{x}$-case the peak is narrow and keeps its zero energy position for every $k_{y}$. The width of the gap is determined by the bulk pair potential $\Delta(\theta)=\Delta_{x} \cos (\theta)$ despite the pair potential at the surface is almost absent. Therefore, the predominant contribution to formation of ARDoS at the surface is provided by the proximity effect with the bulk superconductor.

In contrast, for the chiral symmetry case (Fig.4.8b-d), the energy of a bound state depends linearly on $k_{y}$. The dispersion of the corresponding peak depends on surface 
properties: the higher the roughness, the wider this peak. The value of the gap in the surface DoS is now $k_{y}$ independent and is also determined by proximity with the bulk material. However, for high $k_{y}$ (for the particles moving almost parallel to the surface) it grows up to $\Delta_{y}$ at the surface. In accordance with Fig. 4.1 it provides different properties in the limits of clean and rough surface since the value of $\Delta_{y}$ can be larger or smaller compared to the bulk. All these effects appear in the vicinity of the surface at distances of the order of coherence length (Fig. 4.9a-b).

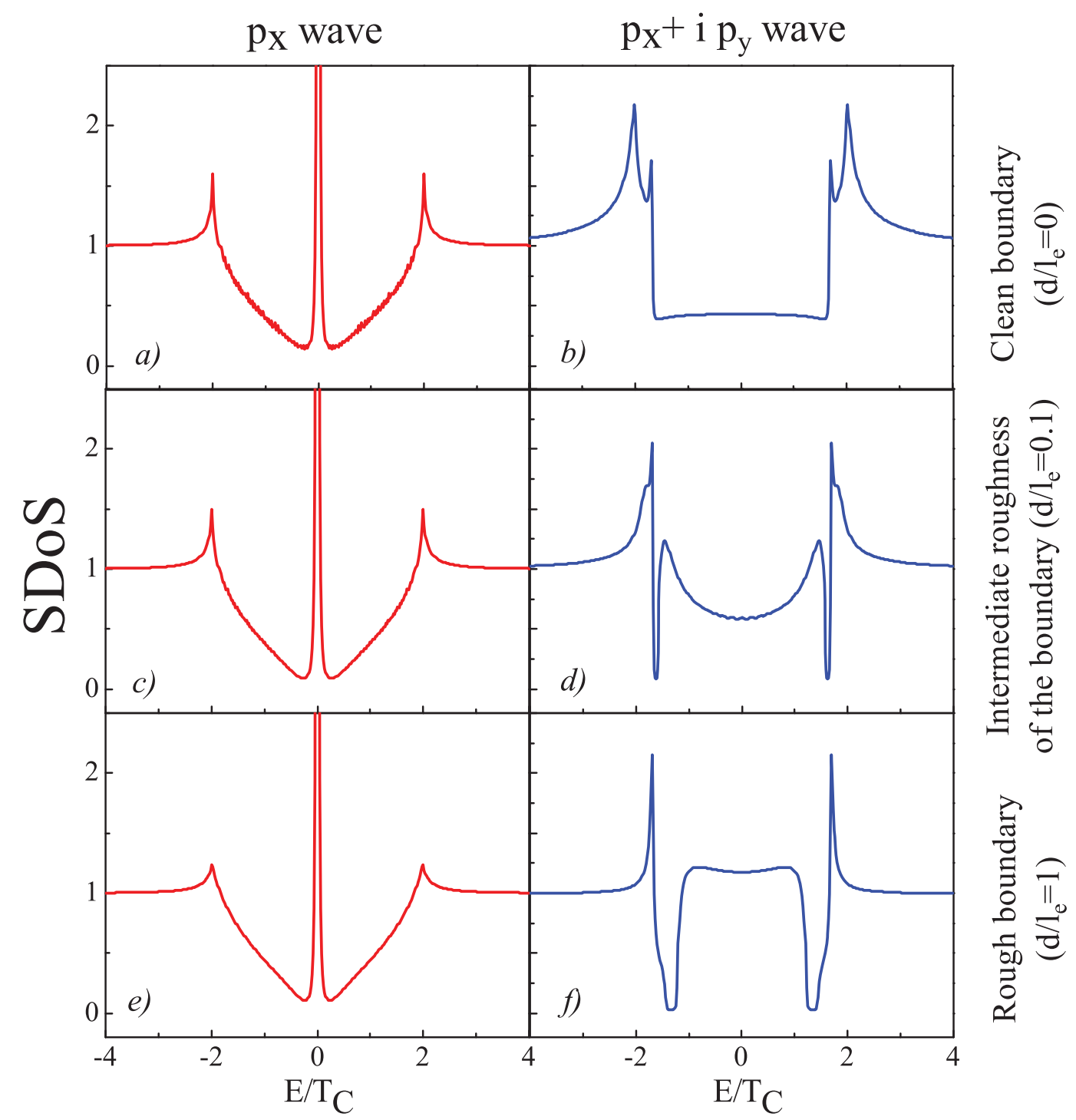

Figure 4.10. Angle Averaged Surface DoS for various values of the roughness parameter: $\left(d / l_{e}=0\right.$ at the upper panels, $d / l_{e}=0.1$ at the middle panels and $d / l_{e}=1$ at the lower panels). Left and right columns of panels correspond to the $p_{x}$ and $p_{x}+i p_{y}$ symmetry, respectively

These properties lead to totally different angle averaged DoS for various symmetries. 
$p_{x}$ superconductor preserves the zero energy peak and peaks at the bulk $\Delta$ even in an averaged surface DoS (SDoS) regardless of the roughness (Fig. 4.10a,c,e). The results for the chiral case strongly depend on properties of the surface. In the case of mirror surface (Fig. 4.10b) angle-averaged subgap SDoS transforms into plateau with a value around zero. The structure above the gap includes two peaks: the intrinsic $\left(E=2 T_{C}\right)$ one provided by the surface component $\Delta_{y}$ of the pair potential and the proximity one $\left(E=1.67 T_{C}\right)$, which appears due to influence of the bulk part of material. However, the growth of roughness leads to suppression of $\Delta_{y}$ and to the shift of intrinsic peak inside the energy range between proximity peaks (Fig. 4.10d). At the same time, the magnitude of the middle plateau grows until it merges with intrinsic peaks in the limit of dirty surface (Fig. 4.10f). It also provides formation of DoS dips between proximity and intrinsic peaks. Dependence of the surface DoS on roughness $d / l_{e}$ is presented in Fig. $4.9 \mathrm{c}-\mathrm{d}$, where it is demonstrated how the intrinsic peak shifts into the gap with an increase of roughness.

\subsection{Conclusion}

In this work we have derived effective boundary conditions at diffusive surface of clean $p$-wave superconductor. Using the developed approach, we study both $p_{x}$ and $p_{x}+$ $i p_{y}$ - wave superconductors with various surface properties ranging from the mirror to heavily rough. We consider the behavior of the most important characteristics of these systems: pair potential $\Delta$, pair amplitudes $f_{1}$ and $f_{2}$ and density of states as a function of surface roughness. In the $p_{x}$ case we demonstrate the robustness of zero-energy peak in the density of states with respect to surface roughness. This effect is due to stability of odd-frequency pairing state at the surface with respect to disorder. In the case of chiral $p_{x}+i p_{y}$ state we demonstrate the appearance of complex multi-peak subgap structure with increasing surface roughness. Furthermore, the systems with a finite surface roughness provide more complicated spectra than in the limits of mirror or heavily rough surfaces. This fact should be taken into account in interpretation of the results of tunneling spectroscopy of unconventional superconductors.

Finally, it is important to note that the robust zero-energy peak in DoS discussed in this work is protected by topology. For example, the topological origin of the flat band on the surface of a d-wave superconductor has been clarified in [30] (see further referencers in [94]). Topologial stability of surface bound states of two-dimensional $p_{x}$ wave and chiral 
p-wave superconductors has been studied in [75,95].

\subsection{Appendix}

\subsubsection{Diffusive layer solution}

The solution of equations (4.13)-(4.14) in the diffuse layer can be represented as [61]

$$
\begin{aligned}
& \frac{F^{+} a_{ \pm}+G-1}{F^{+} a_{ \pm}+G+1}=C_{1 \pm} \exp \{\mp k x\} \\
& \frac{F b_{ \pm}+G-1}{F b_{ \pm}+G+1}=C_{2 \pm} \exp \{ \pm k x\}
\end{aligned}
$$

where $C_{1 \pm}$ and $C_{2 \pm}$ are integration constants

$$
\begin{gathered}
k=\frac{\sqrt{\left\langle g_{+}+g_{-}\right\rangle^{2}+\left\langle f_{+}^{+}+f_{-}^{+}\right\rangle\left\langle f_{+}+f_{-}\right\rangle}}{\ell \cos (\theta)}, \\
F=\frac{\left\langle f_{+}+f_{-}\right\rangle}{\sqrt{\left\langle g_{+}+g_{-}\right\rangle^{2}+\left\langle f_{+}^{+}+f_{-}^{+}\right\rangle\left\langle f_{+}+f_{-}\right\rangle}}, \\
F^{+}=\frac{\left\langle f_{+}^{+}+f_{-}^{+}\right\rangle}{\sqrt{\left\langle g_{+}+g_{-}\right\rangle^{2}+\left\langle f_{+}^{+}+f_{-}^{+}\right\rangle\left\langle f_{+}+f_{-}\right\rangle}} \\
G=\frac{\left\langle g_{+}+g_{-}\right\rangle}{\sqrt{\left\langle g_{+}+g_{-}\right\rangle^{2}+\left\langle f_{+}^{+}+f_{-}^{+}\right\rangle\left\langle f_{+}+f_{-}\right\rangle}} .
\end{gathered}
$$

Integration constants $C_{1 \pm}$ and $C_{2 \pm}$ can easily be expressed in terms of functions $b_{ \pm}$and $a_{ \pm}$ on the boundary of the diffusion layer with a superconductor $(x=0)$. So for the constant $C_{1 \pm}$ and $C_{2 \pm}$ it is possible to get

$$
\begin{gathered}
C_{2-}=\frac{F b_{-}(0, \theta)+G-1}{F b_{-}(0, \theta)+G+1}, \\
C_{1+}=\frac{F^{+} a_{+}(0, \theta)+G-1}{F^{+} a_{+}(0, \theta)+G+1} .
\end{gathered}
$$

Substituting (4.36), (4.37) into the solution (4.32), (4.33) for the functions $b_{-}(d, \theta)$ and $a_{+}(d, \theta)$ at the free surface of the diffusion layer we get

$$
b_{-}(d, \theta)=-\frac{G}{F}+\frac{1}{F} \frac{1+p}{1-p}, p=C_{2-} \exp \{-k d\}
$$




$$
a_{+}(d, \theta)=-\frac{G}{F^{+}}+\frac{1}{F^{+}} \frac{1+q}{1-q}, q=C_{1+} \exp \{-k d\}
$$

Proceeding in a similar way, it is easy to see that

$$
\begin{gathered}
a_{-}(0,-\theta)=-\frac{G}{F^{+}}+\frac{1}{F^{+}} \frac{1+u}{1-u}, \\
b_{+}(0,-\theta)=-\frac{G}{F}+\frac{1}{F} \frac{1+v}{1-v},
\end{gathered}
$$

where

$$
\begin{aligned}
& u=\exp \{-k d\} \frac{F^{+} a_{+}(d, \theta)+G-1}{F^{+} a_{+}(d, \theta)+G+1}=q \exp \{-k d\} \\
& v=\exp \{-k d\} \frac{F b_{-}(d, \theta)+G-1}{F b_{-}(d, \theta)+G+1}=p \exp \{-k d\}
\end{aligned}
$$

The resulting equations (4.36)-(4.43) and boundary conditions (4.17), (4.18) set the desired relation between the functions of a coming $a_{+}(0,-\theta), b_{-}(0,-\theta)$ and leaving $a_{-}(0, \theta), b_{+}(0, \theta)$ of the diffusion layer

$$
\begin{aligned}
& a_{-}(0, \theta)=\frac{a_{+}(0,-\theta)-\left(G a_{+}(0,-\theta)-F\right) \tanh \{k d\}}{\left(F^{+} a_{+}(0,-\theta)+G\right) \tanh \{k d\}+1}, \\
& b_{+}(0, \theta)=\frac{b_{-}(0,-\theta)-\left(G b_{-}(0,-\theta)-F^{+}\right) \tanh \{k d\}}{\left(F b_{-}(0,-\theta)+G\right) \tanh \{k d\}+1} .
\end{aligned}
$$

From relations (4.44), (4.45), it follows that (as in d-wave case [61]) the values of the modified functions Eilenberger on leaving the border trajectory can be divided into two parts. One of them

$$
a_{-, d}(0, \theta)=\frac{F}{1+G}, b_{+, d}(0, \theta)=\frac{F^{+}}{1+G}
$$

is determined by the uncorrelated contribution to the direction of the angle $\theta$. It is formed as a result of rescattering in this corner of particles incident on the diffuse layer in the whole range of trajectories towards this layer. It is easy to see that this part defines the functions of $a_{-}(0, \theta)$ and $b_{+}(0, \theta)$ in the limit of large thickness of the diffusion layer, $k d \gg 1$. In this case, the electrons incident and reflected from the surface are completely uncorrelated. The remaining parts

$$
\begin{gathered}
b_{+, c}(0, \theta)=\frac{(1-\tanh (k d))\left((1+G) b_{-}(0,-\theta)-F^{+}\right)}{(1+G)\left(\left(F b_{-}(0,-\theta)+G\right) \tanh \{k d\}+1\right)}, \\
a_{-, c}(0, \theta)=\frac{(1-\tanh (k d))\left((1+G) a_{+}(0,-\theta)-F\right)}{(1+G)\left(\left(F^{+} a_{+}(0,-\theta)+G\right) \tanh \{k d\}+1\right)},
\end{gathered}
$$


set the degree of correlation between the incoming and outgoing from the boundary trajectories. It is evident that this correlation is stronger, the smaller the thickness of the diffusion layer $d$. Indeed, from (4.47), (4.48), it follows that at angles

$$
\frac{\pi}{2} \leq \theta \leq \arccos \left(\frac{d \sqrt{\left\langle g_{+}+g_{-}\right\rangle^{2}+\left\langle f_{+}^{+}+f_{-}^{+}\right\rangle\left\langle f_{+}+f_{-}\right\rangle}}{l}\right)
$$

scattering is mainly diffusive. With decreasing thickness, this region of angles shrinks, so that in the limit of small thickness $(k d \ll 1)$ it is more and more limited by trajectories, moving along the border. As a rule, they do not contribute to physical observables (DoS, the conductance, the critical current of Josephson junctions). For all other paths that define these values, the boundary conditions (4.44), (4.45) reduce in this limit to the mirror (4.17), (4.18) type.

\subsubsection{Symmetry relations}

The system of Eilenberger equations is

$$
\begin{gathered}
2 \omega f_{\omega}(x, \theta)+v \cos (\theta) \frac{d}{d x} f_{\omega}(x, \theta)=2 \Delta g_{\omega}(x, \theta), \\
2 \omega f_{\omega}^{+}(x, \theta)-v \cos (\theta) \frac{d}{d x} f_{\omega}^{+}(x, \theta)=2 \Delta^{*} g_{\omega}(x, \theta), \\
2 v \cos (\theta) \frac{d}{d x} g_{\omega}(x, \theta)=2\left(\Delta^{*} f_{\omega}-\Delta f_{\omega}^{+}\right) . \\
\Delta=\Delta_{x} \cos \theta+i \Delta_{y} \sin \theta
\end{gathered}
$$

First we consider relations with respect to $\theta$. We write them for angle $-\theta$ and conjugate, resulting in

$$
\begin{gathered}
2 \omega f_{\omega}^{*}(-\theta)+v \cos (\theta) \frac{d}{d x} f_{\omega}^{*}(-\theta)=2 \Delta g_{\omega}^{*}(-\theta), \\
2 \omega f_{\omega}^{+*}(-\theta)-v \cos (\theta) \frac{d}{d x} f_{\omega}^{+*}(-\theta)=2 \Delta^{*} g_{\omega}^{*}(-\theta), \\
2 v \cos (\theta) \frac{d}{d x} g_{\omega}^{*}(-\theta)=2\left(\Delta^{*} f_{\omega}^{*}(-\theta)-\Delta f_{\omega}^{+*}(-\theta)\right) .
\end{gathered}
$$


This set of equations coinsides with initial one after the following substitution

$$
\begin{aligned}
g_{\omega}(\theta) & =g_{\omega}^{*}(-\theta) \\
f_{\omega}(\theta) & =f_{\omega}^{*}(-\theta) \\
f_{\omega}^{+}(\theta) & =f_{\omega}^{+*}(-\theta)
\end{aligned}
$$

This proves angle-symmetry relations (4.30).

Next, we consider symmetry of Eilenberger equations with respect to Matsubara frequency $\omega$. Similarly to the previous step, we take equations at negative frequncy $-\omega$ and conjugate them. After conjugation and some rearrangements we arrive

$$
\begin{gathered}
2 \omega f_{-\omega}^{*}(\theta)-v \cos (\theta) \frac{d}{d x} f_{-\omega}^{*}(\theta)=2 \Delta^{*}\left(-g_{-\omega}^{*}(\theta)\right), \\
2 \omega f_{-\omega}^{+*}(\theta)+v \cos (\theta) \frac{d}{d x} f_{-\omega}^{+*}(\theta)=2 \Delta\left(-g_{-\omega}^{*}(\theta)\right), \\
2 v \cos (\theta) \frac{d}{d x}\left(-g_{-\omega}^{*}(\theta)\right)=2\left(\Delta^{*} f_{-\omega}^{+*}-\Delta f_{-\omega}^{*}\right) .
\end{gathered}
$$

Comparison with initial equations provides the required symmetry relations (4.29).

$$
\begin{gathered}
g_{-\omega}(\theta)=-g_{\omega}^{*}(\theta) \\
f_{-\omega}(\theta)=f_{\omega}^{+*}(\theta) \\
f_{-\omega}^{+}(\theta)=f_{\omega}^{*}(\theta)
\end{gathered}
$$




\section{Bibliography}

[1] Y. Maeno, H. Hashimoto, K. Yoshida, S. Nishizaki, T. Fujita, J. G. Bednorz, and F. Lichtenberg, Nature (London) 372, 532 (1994)

[2] K. Ishida, H. Mukuda, Y. Kitaoka, K. Asayama, Z. Q.Mao, Y. Mori, and Y. Maeno, Nature (London) 396, 658 (1998)

[3] G. M. Luke, Y. Fudamoto, K. M. Kojima, M. I. Larkin, J.Merrin, B. Nachumi, Y. J. Uemura, Y. Maeno, Z. Q. Mao, Y. Mori, H. Nakamura, and M. Sigrist, Nature (London) 394, 558 (1998)

[4] A. P. Mackenzie and Y. Maeno, Rev. Mod. Phys. 75, 657 (2003)

[5] K. D. Nelson, Z. Q. Mao, Y. Maeno and Y. Liu, Science 306, 1151 (2004)

[6] Y. Asano, Y. Tanaka, M. Sigrist and S. Kashiwaya, Phys.Rev. B 67, 184505 (2003); Phys. Rev. B 71, 214501 (2005).

[7] H. Tou, Y. Kitaoka, K. Ishida, K. Asayama, N. Kimura, Y. Onuki, E. Yamamoto, Y. Haga and K. Maezawa, Phys.Rev. Lett. 80, 3129 (1998)

[8] V. Muller, Ch. Roth, D. Maurer, E. W. Scheidt, K. Lers, E.Bucher and H. E. Bmel, Phys. Rev. Lett. 58, 1224 (1987)

[9] Y. J. Qian, M. F. Xu, A. Schenstrom, H. P. Baum, J. B.Ketterson, D. Hinks, M. Levy and B. K. Sarma, Solid State Commun., 63, 599 (1987)

[10] A. A. Abrikosov, J. of Low Temp. Phys. 53, 359 (1983)

[11] H. Fukuyama and Y. Hasegawa, J. Phys. Soc. Jpn. 56, 8777 (1987)

[12] A. G. Lebed, K. Machida and M. Ozaki, Phys. Rev. B 62, 795 (2000)

[13] S. S. Saxena, P. Agarwal, K. Ahilan, F. M. Grosche, R.K. W. Haselwimmer, M. J. Steiner, E. Pugh, I. R. Walker, S. R. Julian, P. Monthoux, G. G. Lonzarich, A. Huxley, I.Shelkin, D. Braithwaite, and J. Flouquet, Nature (London) 406, 587 (2000) 
[14] C. Peiderer, M. Uhlarz, S. M. Hayden, R. Vollmer, H. v.Lohneysen, N. R. Bernhoeft, and G. G. Lonzarich, Nature (London) 412, 58 (2001)

[15] D. Aoki, A. Huxley, E. Ressouche, D. Braithwaite, J. Flouquet, J. Brison, E. Lhotel, and C. Paulsen, Nature (London) 413, 613 (2001)

[16] N. Kikugawa, K. Deguchi, and Y. Maeno, Physica C 388, 483 (2003); K.Deguchi, Z. Q. Mao, H. Yaguchi, and Y. Maeno, Phys. Rev. Lett. 92, 047002 (2004)

[17] M. J. Graf, S. K. Yip and J. A. Sauls, Phys. Rev. B, 62, 14393 (2000)

[18] K. Machida, T. Nishira, and T. Ohmi, J. Phys. Soc. Jpn, 68, 3364 (1999)

[19] B. Lussier, B. Ellman and L. Taillefer, Phys. Rev. B, 53, 5145 (1996)

[20] J. Alicea, Phys. Rev. B 81, 125318 (2010)

[21] Jay D. Sau, Roman M. Lutchyn, Sumanta Tewari, and S. Das Sarma Phys. Rev. Lett. 104, $040502(2010)$

[22] Jay D. Sau, Roman M. Lutchyn, Sumanta Tewari, and S. Das Sarma, Phys. Rev. B, $82,094522(2010)$

[23] Yamakage, Ai and Tanaka, Yukio and Nagaosa, Naoto, Phys. Rev. Lett. 108, 087003 (2012)

[24] L. Fu and C. L. Kane, Phys. Rev. Lett. 100, 096407 (2008)

[25] A. R. Akhmerov and J. Nilsson and C. W. J. Beenakker, Phys. Rev. Lett. 102, 216404 (2009)

[26] Tanaka, Yukio and Yokoyama, Takehito and Nagaosa, Naoto, Phys. Rev. Lett. 103, $107002(2009)$

[27] K. T. Law and P. A. Lee and T. K. Ng, Phys. Rev. Lett. 103, 237001 (2009)

[28] Lutchyn, Roman M. and Sau, Jay D. and Das Sarma, S. Phys. Rev. Lett. 105, 077001 (2010)

[29] Oreg, Yuval and Refael, Gil and von Oppen, Felix, Phys. Rev. Lett. 105, 177002 (2010) 
[30] A. P. Schnyder, S. Ryu, A. Furusaki, and A. W. W. Ludwig, Phys. Rev. B 78195125 (2008)

[31] J. Alicea, Rep. Prog. Phys. 75076501 (2012)

[32] X.-L. Qi and S.-C. Zhang, Rev. Mod. Phys. 831057 (2011)

[33] Y. Tanaka, M. Sato, and N. Nagaosa, J. Phys. Soc. Jpn. 81011013 (2012)

[34] L. J. Buchholtz and G. Zwicknagl, Phys. Rev. B 23, 5788 (1981)

[35] J. Hara and K. Nagai, Prog. Theor. Phys. 76, 1237 (1986)

[36] C.-R. Hu, Phys. Rev. Lett. 72, 1526 (1994)

[37] S. Kashiwaya and Y. Tanaka, Rep. Prog. Phys. 63, 1641 (2000)

[38] K. Yada, A. Golubov, Y. Tanaka, S. Kashiwaya, J. Phys. Soc. Jpn. 83 (2014) 074706 (2014)

[39] M. Sato, Y. Tanaka, K. Yada, and T. Yokoyama, Phys. Rev. B 83 (2011) 224511.

[40] Y. Tanaka and S. Kashiwaya, Phys. Rev. Lett. 74, 3451 (1995); Phys. Rev. B 53, 11957 (1996)

[41] T. Lofwander, V. S. Shumeiko, and G. Wendin, Supercond. Sci.Technol. 14, R53 (2001)

[42] S. Kashiwaya, Y. Tanaka, M. Koyanagi, H. Takashima, and K. Kajimura, Phys. Rev. B 511350 (1995) ; S. Kashiwaya, Y. Tanaka, N. Terada, M. Koyanagi, S. Ueno, L. Alff, H. Takashima, Y. Tanuma, and K. Kajimura, J. Phys. Chem. Solids 592034 (1998)

[43] M. Covington, M. Aprili, E. Paraoanu, L. H. Greene, F. Xu, J. Zhu, and C. A. Mirkin, Phys. Rev. Lett. 79277 (1997)

[44] L. Alff, H. Takashima, S. Kashiwaya, N. Terada, H. Ihara, Y. Tanaka, M. Koyanagi, and K. Kajimura, Phys. Rev. B 55 R14757 (1997)

[45] J. Y. T. Wei, N.-C. Yeh, D. F. Garrigus, and M. Strasik, Phys. Rev. Lett. 812542 (1998) 
[46] I. Iguchi, W. Wang, M. Yamazaki, Y. Tanaka, and S. Kashiwaya, Phys. Rev. B 62 R6131 (2000)

[47] A. Biswas, P. Fournier, M. M. Qazilbash, V. N. Smolyaninova, H. Balci, and R. L. Greene, Phys. Rev. Lett. 88207004 (2002)

[48] B. Chesca, H. J. H. Smilde, and H. Hilgenkamp, Phys. Rev. B 77184510 (2008)

[49] Y. Nagato and K. Nagai, Phys. Rev. B 51, 16254 (1995)

[50] L. J. Buchholtz, M. Palumbo, D. Rainer, and J. A. Sauls, J. Low Temp. Phys. 101, 1079 (1995)

[51] Yu. S. Barash, A. A. Svidzinsky, and H. Burkhardt, Phys. Rev. B 55, 15282 (1997)

[52] Y. Tanuma, Y. Tanaka, M. Yamashiro, and S. Kashiwaya, Phys. Rev. B 57, 7997 (1998)

[53] M. Matsumoto and H. Shiba, J. Phys. Soc. Jpn. 64, 3384 (1995); 64, 4867 (1995); 65, $2194(1996)$

[54] M. Fogelstrom, D. Rainer, and J. A. Sauls, Phys. Rev. Lett. 79, 281 (1997)

[55] Y. Tanuma, Y. Tanaka, and S. Kashiwaya, Phys. Rev. B 64, 214519 (2001)

[56] L. J. Buchholtz, Phys. Rev. B 33, 1579 (1986)

[57] W. Zhang, J. Kurkijärvi, and E. V. Thuneberg, Phys. Rev. B 36, 1987 (1987); W. Zhang, Phys. Lett. A, 130, 4, 314 (1988)

[58] Y. Nagato, M. Yamamoto, and K. Nagai, J. Low Temp. Phys. 110, 1135 (1998)

[59] A. A. Golubov and M. Yu. Kupriyanov, Pis'ma Zh. Eksp. Teor. Fiz. 67, 478 ( 1998) [JETP Lett 67, 501 (1998)]

[60] A. A. Golubov and M. Yu. Kupriyanov, Superlattices and Microstructures, 25, 949 (1999)

[61] A. A. Golubov and M. Yu. Kupriyanov, Pis'ma Zh. Eksp. Teor. Fiz. 69, 242 ( 1999) [JETP Lett. 69, 262 (1999)] 
[62] Y. Tanaka, Y. V. Nazarov, and S. Kashiwaya, Phys. Rev. Lett. 90, 167003 (2003); Y. Tanaka, Y. V. Nazarov, A. A. Golubov, and S.Kashiwaya, Phys. Rev. B 69, 144519 (2004)

[63] V. L. Berezinskii, Pis'ma Zh. Eksp. Teor. Fiz. 20, 628 (1974) [JETP Lett. 20, 287 (1974)]

[64] Y. Tanaka, Y. Tanuma and A. A. Golubov, Phys. Rev. B 76, 054522 (2007)

[65] M. Eschrig, T. Löfwander, T. Champel, J. Cuevas, and G.Schön, J. Low. Temp. Phys. 147, $457(2007)$

[66] Y. Tanaka and A. A. Golubov, Phys. Rev. Lett. 98, 037003 (2007)

[67] Y. Tanaka and S. Kashiwaya, Phys. Rev. B 70, 012507 (2004)

[68] Y. Tanaka, Y. Asano, A. A. Golubov, and S.Kashiwaya, Phys. Rev. B 72, 140503 $(2005)$

[69] Y. Asano, Y. Tanaka, and S. Kashiwaya, Phys. Rev. Lett. 96, 097007 (2006)

[70] Y. Asano, Y. Tanaka, A. A. Golubov, and S. Kashiwaya Phys. Rev. Lett. 99, 067005 (2007).

[71] Y. V. Fominov, Pis'ma Zh. Eksp. Teor. Fiz. 86, 842 (1997) [JETP Lett. 86, 732 (2007)]

[72] Y. Asano, A.A. Golubov, Y. Fominov, and Y. Tanaka, Phys. Rev. Lett. 107, 087001 (2011)

[73] S. Higashitani, H. Takeuchi, S. Matsuo, Y. Nagato, and K. Nagai, Phys. Rev. Lett. 110, $175301(2013)$

[74] A. Keles, A. Andreev, S. Kivelson, and B. Spivak, arxiv:1405.7090 (2014)

[75] A. Furusaki, M. Matsumoto, and M. Sigrist: Phys. Rev. B 64, 054514 (2001)

[76] M. Matsumoto and M. Sigrist, J. Phys. Soc. Jpn. 68, 994 (1999)

[77] M. Yamashiro, Y. Tanaka, and S. Kashiwaya, Phys. Rev. B 56 (1997) 7847; M. Yamashiro, Y. Tanaka, Y. Tanuma, and S. Kashiwaya, J. Phys. Soc. Jpn. 673224 (1998) 
[78] C. Honerkamp and M. Sigrist, J. Low Temp. Phys. 111895 (1998)

[79] K. Sengupta, H.-J.Kwon, and V. M. Yakovenko, Phys. Rev.B 65104504 (2002)

[80] F. Laube, G. Goll, H. v. Löhneysen, M. Fogelström, and F. Lichtenberg, Phys. Rev. Lett. 841595 (2000)

[81] S. Kashiwaya, H. Kashiwaya, H. Kambara, T. Furuta, H. Yaguchi, Y. Tanaka, and Y. Maeno, Phys. Rev. Lett. 107, 077003 (2011)

[82] S. Higashitani, S. Matsuo, Y. Nagato, and K. Nagai, S. Murakawa, R. Nomura, and Y. Okuda, Phys. Rev. B 85, 024524 (2012).

[83] K. Nagai, Y. Nagato, M. Yamamoto and S. Higashitani, J. Phys. Soc. Jpn. 77, 111003 (2008).

[84] W. Zhang, Phys. Lett. A, 130, 4, 314 (1988).

[85] M. Matsumoto, M. Koga and H. Kusunose, J. Phys. Soc. Jpn. 82, 034708 (2013).

[86] Y. Nagato, S. Higashitani and K. Nagai, J. Phys. Soc. Jpn. 80, 113706 (2011).

[87] G. Eilenberger, Z. Phys. 214, 195 (1968).

[88] C. Bruder, Phys. Rev. B 41, 4017 (1990).

[89] V.P Mineev, arXiv:1402.2111.

[90] R. Sh. Askhadullin, V. V. Dmitriev, D. A. Krasnikhin, P. N. Martynov, A. A. Osipov, A. A. Senin, A. N. Yudin, Pisma v ZhETF, 95, 355 (2012) [JETP Lett. 95, 326 (2012)].

[91] Yu. N. Ovchinnikov, Zh. Eksp. Teor. Fiz. 56, 1590 (1969) [Sov. Phys. JETP 29, 853 (1969)].

[92] N. Schopohl and K. Maki, Phys. Rev. B 52, 490 (1995).

[93] S. Ryu and Y. Hatsugai in PRL 89, 077002 (2002)

[94] M. Silaev, G.E. Volovik, arXiv:1405.1007.

[95] M. Sato, Y. Tanaka, K. Yada, and T. Yokoyama, Phys. Rev. B, 83, 224511, (2011) 


\section{Summary}

This thesis is devoted to the study of controllable proximity effects in superconductors, both in terms of fundamental aspects and applications. As a part of this thesis theoretical description was suggested for a number of structures with superconducting electrodes and multiple interlayers. These structures include new physics related to the proximity effect.

In spite of the fact, that the physical foundations of the proximity effect are well known and described in literature, the aim of this work is to find solutions for the problems of the superconducting electronics.

Chapter 1 is devoted to consideration of nanoscale $\varphi$ - junctions. They are Josephson devices with nontrivial phase difference $0<\varphi_{g}<\pi$ in the ground state. $\varphi$-junctions can be realized in the structures composed from longitudinally oriented normal metal $(\mathrm{N})$ and ferromagnetics (F) films in the weak link region. Oscillatory coupling across F-layer makes the first harmonic in the current-phase relation relatively small, while coupling across Nlayer provides negative sign of the second harmonic. To derive a quantitative criteria for a $\varphi$-junction two-dimensional boundary-value problem has been solved in the frame of Usadel equations for overlap and ramp geometries of S-NF-S structures.

This chapter is focused on different geometries of nanoscale structures of the size much less than Josephson penetration depth $\lambda_{J}$. We have shown that stable $\varphi$-state can be realized in S-NF-S structures with longitudinally oriented NF-bilayers. At the same time $\varphi$ state can not be achieved in conventional SNS and SFS structures. Proximity effect between $\mathrm{N}$ and $\mathrm{F}$ layer limits minimal possible size of $\varphi$-junction. In the case of smaller junctions, NF-bilayer becomes almost homogeneous, $\varphi$-state is prohibited and junction exists in 0 - or $\pi$-state. The conditions for realization of $\varphi$-state in ramp-type S-NF-S, overlap-type SFNFN-NFS and RTO-type SN-FN-NS geometries are discussed in Chapter 1. It is shown that RTO-type SN-FN-NS geometry is most suitable for practical realisation. It is also shown in this chapter, that the parameter range of $\varphi$-state existence can be sufficiently broadened. It allows to realize $\varphi$ - Josephson junctions using up-to-date technology.

Temperature variation slightly shifts the interval of relevant $0-\pi$ transition and per- 
mits one to tune the desired ground state phase. Furthermore, sensitivity of the ground state to an electron distribution function permits applications of $\varphi$-junctions as small-scale self-biasing single-photon detectors. Moreover these junctions are controllable and have degenerate ground states $+\varphi$ and $-\varphi$, providing necessary condition for quantum bits and quantum detectors.

Chapters 2 and 3 are devoted to theoretical study of SIsFS junctions where 'S' is a bulk superconductor and 'IsF' is a complex weak link consisting of a superconducting film 's', a metallic ferromagnet ' $\mathrm{F}$ ' and an insulating barrier 'I'. At temperatures close to the critical temperature, $T \lesssim T_{C}$, calculations are performed analytically in the frame of the Ginsburg-Landau equations. The numerical method is developed to solve this problem self-consistently for arbitrary temperature.

Chapter 2 demonstrates various modes of operation in SIsFS junctions. The key element in their operation is a state of thin s-layer, where superconductivity is controlled by inverse proximity effect. This phenomenon allows one to propose a new way to make logic or memory element. Superconductive and Normal states of s-layer become good logical states of such device, because critical currents through the structure in these states are significantly different.

We have shown that magnitude of $I_{C} R_{N}$ product of SIsFS junction is on the level of ideal tunnel junction SIS and significantly exceeds the characteristic voltage of other ferromagnetic junctions. Furthermore high value of $I_{C} R_{N}$ product can be reached even in the $\pi$-state. It means that such junction can be used as an effective $\pi$-shifter.

In Chapter 2 the Current Phase Relation (CPR) of SIsFS junction is considered in different modes of operation. When s-layer goes into the normal state, CPR is almost sinusoidal. However, in superconductive mode, the presence of two separated parts of weak link leads to the formation of nonsinosidual current phase relations with the amplitude of second harmonic of CPR around $40 \%$ from the first harmonic even at high temperatures. At low temperatures the intrinsic distortion of CPR of SFS junctions also occurs, and the magnitude of the second harmonic becomes comparable to the first one.

Chapter 3 is devoted to the comparison with measurements performed for SIsFS junctions by HYPRES (USA) and ISSP RAS (Chernogolovka, Russia) groups. Qualitative agreement between theoretical and experimental temperature and field dependencies of $I_{C}(H)$ is demonstrated. The switching between two modes of operation is shown on the temperature dependence of critical current. Critical current of the whole structure starts 
to grow during crossing of effective critical temperature of the s-layer.

Measurements in magnetic field show that remagnitization of the F-layer shifts maximum of $I_{C}(H)$ dependence and provides another basis for logic element. However this approach for development of memory is not scalable. Further miniaturization requires implementation of other logic basis, for example the one proposed in Chapter 2.

Chapter 4 presents the results of theoretical study of surface properties in a twodimensional model for triplet $p$-wave superconductors. Boundary conditions are derived in terms of Eilenberger equations at rough interfaces. The approach is developed for selfconsistent solution for the spatial dependence of $p_{x}$ and $p_{x}+i p_{y}$-wave pair potentials. In the $p_{x}$ case it was demonstrated that pair potential $\Delta_{x}$ is strongly suppressed in the vicinity of surface. Significant energy gap in the electronic density of states occurs only due to proximity with a bulk superconductor. However, the robustness of the zero-energy peak in the density of states (DoS) with respect to surface roughness was shown, in contrast to the suppression of such a peak in the case of $d_{x y}$ symmetry. This effect is due to stability of odd-frequency pairing state generated at the surface with respect to disorder.

In the case of the chiral $p_{x}+i p_{y}$ state tangential component of pair potential $\Delta_{y}$ is conserved at the boundary, while the normal one $\Delta_{x}$ is strongly suppressed similar to $p_{x}$-case. Moreover, the surface roughness and respective isotropization of pair amplitude significantly decrease the magnitude of $\Delta_{y}$. It leads to the appearance of a complex multipeak subgap structure in the spectrum with increasing surface roughness. Furthermore, systems with a finite surface roughness provide more complex spectra compared to mirror or strongly disordered surfaces. This fact should be taken into account in interpretation of the results of tunneling spectroscopy of unconventional superconductors.

All the phenomena considered in Chapters 1-4 are related to the study of proximity effect. In the Chapter 1 the prior consideration was given to proximity between ferromagnetic and normal layers inside weak link. This proximity effect determines the current redistribution between conductance channels across the junction. Chapters 2-3 were devoted to proximity of thin superconductive layer s with F-layer and bulk S-electrodes. The interaction with F-layer suppresses superconductivity in the interlayer while interaction with S-electrodes supports it. Thus a state of middle s-film is crucial for the operation of the whole structure. Finally in Chapter 4 the attention was focused on proximity effect between different lobes of p-wave order parameter connected with each other by rough surface. All the physical systems mentioned above have much similarity in the descrip- 
tion. The study of proximity effect in these systems permits to develop more effective and controllable devices for superconductive electronics. 


\section{Samenvatting}

Deze thesis is gewijd aan die studie naar controleerbare nabijheidseffecten in supergeleiders, zowel in termen van fundamentele aspecten als in applicaties. Als onderdeel van deze thesis wordt een theoretische beschrijving voor een aantal structuren met supergeleidende elektroden en meerdere lagen voorgesteld. Deze structuren bevatten nieuwe natuurkunde die verband houdt met het nabijheidseffect.

Ondanks het feit dat de natuurkundige grondslagen van het nabijheidseffect alom bekend zijn en in de literatuur beschreven zijn, is het doel van dit werk om problemen met de supergeleidende elektronica op te lossen.

Hoofdstuk 1 is gewijd aan de beschouwing van $\varphi$-juncties op nanoschaal. Dit zijn Josephsonapparaatjes met een niet-triviaal faseverschil $0<\varphi_{g}<\pi$ in de grondtoestand. $\varphi$ juncties kunnen gerealiseerd worden in structuren die opgebouwd zijn uit in de lengterichting geori $\Gamma$ «nteerde dunne lagen van een normaal metaal $(\mathrm{N})$ en een ferromagneet $(\mathrm{F})$ in het zwakke-schakel-regime. Oscillerende koppeling in de F-laag zorgt voor een relatief kleine eerste harmonische in de stroom-faserelatie, terwijl koppeling in de N-laag een negatieve waarde voor de tweede harmonische veroorzaakt. Om een kwantitatief criterium voor een $\varphi$-junctie af te leiden, is een twee-dimensionaal grenswaardeprobleem opgelost in het kader van de Usadelvergelijkingen voor overlap- en hellingsgeometrien voor S-NF-S-structuren.

Dit hoofdstuk richt zich op verschillende geometrien van nanostructuren van een veel kleiner formaat dan de Josephsonpenetratielengte $\lambda_{J}$. We hebben laten zien dat een stabiele $\varphi$-toestand gerealiseerd kan worden in S-NF-S-structuren met in de lengterichting georienteerde NF-dubbellagen. Tegelijkertijd kan de $\varphi$-toestand niet worden bewerkstelligd in gebruikelijke SNS- en SFS-structuren. Het nabijheidseffect tussen de N- en F-laag beperkt de minimaal mogelijke schaal van de $\varphi$-junctie. In het geval van een te kleine junctie wordt de NF-dubbellaag bijna homogeen, is de $\varphi$-toestand verboden en verkeerd de junctie in de 0 - of de $\pi$-toestand. De voorwaarden voor de realisatie van de $\varphi$-toestand in hellingstype S-NS-S-, overlaptype SFN-FN-NFS- en RTO-type SN-FN-NS-geometrie worden besproken in hoofdstuk 1. Er wordt aangetoond dat de RTO-type SN-FN-NS-geometrie het meest geschikt voor praktische realisatie is. Er wordt in dit hoofdstuk ook weergeven dat het 
parameterbereik voor het bestaan van de $\varphi$-toestand voldoende verbreed kan worden. Dit maakt het mogelijk om $\varphi$-Josephsonjuncties met de hedendaagse technologie te realiseren.

Temperatuurvariatie zorgt voor een lichte verschuiving in het interval met een relevante $0-\pi$-transitie en maakt het mogelijk dat men de gewenste grondtoestandfase kan afstellen. Bovendien staat de gevoeligheid van de grondtoestand voor een elektrondistributiefunctie $\varphi$-junctie-applicaties als kleinschalige enkel-elektrondetectors met zelfregelende instelspanning toe. Daarnaast zijn deze juncties controleerbaar en hebben ze ontaarde grondtoestanden $+\varphi$ and $-\varphi$, zodat ze voorzien in de benodigde voorwaarde voor kwantumbits en kwantumdetectoren.

Hoofdstukken 2 en 3 zijn gewijd aan een theoretische studie naar SIsFS-juncties, waarbij 'S' staat voor een bulk supergeleider en 'IsF' staat voor een samengestelde zwakke schakeling bestaande uit een supergeleidende dunne laag ' $\mathrm{s}$ ', een metallische ferromagneet ' $\mathrm{F}$ ' en een isolerende barriere ' $\mathrm{I}$ '. Bij temperaturen die de kritische temperatuur naderen, $T \lesssim T_{C}$, zijn analytische berekeningen in het kader van de Ginsburg-Landau-vergelijkingen uitgevoerd. De numerieke methode is ontwikkeld om dit probleem op te lossen voor arbitraire temperaturen. Hoofdstuk 2 demonstreert verschillende werkingstoestanden van de SIsFS-juncties. Het sleutelelement in hun werking is een toestand met een dunne slaag waarbij supergeleiding gecontroleerd wordt door het omgekeerde nabijheidseffect. Dit verschijnsel opent nieuwe wegen voor het maken van logische of geheugenelementen. Supergeleidende en normale toestanden van de s-laag vormen goede logische toestanden van dergelijke apparaatjes, omdat kritieke stromen door de structuren in deze toestanden significant verschillend zijn. We hebben gedemonstreerd dat de omvang van het $I_{C} R_{N}$-product van de SIsFS vergelijkbaar met dat van de ideale tunneljunctie SIS is en het karakteristieke voltage van de andere ferromagnetische juncties significant overschrijdt. Bovendien kan een hoge waarde voor het $I_{C} R_{N}$ zelfs in de $\pi$-toestand bereikt worden. Dit betekent dat een dergelijke junctie gebruikt kan worden als een effectieve $\pi$-versteller.

In hoofdstuk 2 wordt de stroom-faserelatie (CPR) van de SIsFS-junctie, beschouwd in verschillende werkingstoestanden. Voor de s-laag in de normale toestand is de CPR bijna sinusvormig. In de supergeleidende toestand leidt de aanwezigheid van twee gescheiden delen van de zwakke schakel echter tot de vorming van niet-sinusvormige stroom-faserelaties waarbij de kritieke amplitude van de tweede harmonische van de CPR rond de $40 \%$ van die van de eerste harmonische zit, zelfs bij hoge temperaturen. In de lage-temperatuurlimiet verschijnt ook de intrinsieke verstoring van de CPR van SFS-juncties, en wordt de omvang 
van de tweede harmonische vergelijkbaar met die van de omvang van de eerste. Hoofdstuk 3 is gewijd aan de vergelijking met metingen aan SIsFS-juncties die uitgevoerd zijn bij groepen van HYPRES (VS) en ISSP RAS (Chernogolovka, Rusland). Kwalitatieve overeenstemming tussen theoretische en experimentele temperatuur- en veldafhankelijkheden $I_{C}(H)$ is hier aangetoond. Er wordt getoond hoe de temperatuurafhankelijkheid van de kritieke stroom leidt tot de overgang tussen twee werkingstoestanden. De kritieke stroom van de hele constructie begint op te lopen bij het doorkruisen van de effectieve kritieke temperatuur van de s-laag.

Metingen van het magnetisch veld laten zien dat demagnetisatie het maximum van de $I_{C}(H)$-afhankelijkheid verschuift en een andere basis voor een logisch element verschaft. Deze aanpak voor het ontwikkelen van geheugen is echter niet op te schalen. Verdere verkleining vereist de verwezenlijking van een andere logische basis, zoals bijvoorbeeld het voorbeeld dat wordt voorgesteld in hoofdstuk 2.

Hoofdstuk 4 laat de resultaten van een theoretische studie naar de eigenschappen van de oppervlaktetoestanden in een twee-dimensionaal model voor triplet-p-golf-supergeleiders zien. Randvoorwaarden zijn afgeleid in termen van Eilenbergervergelijkingen voor ruwe grensvlakken. Deze aanpak is ontwikkeld om een zelf-consistente oplossing voor de plaatsafhankelijkheid van de $p_{x}$ and $p_{x}+i p_{y}$-golfpaarpotentialen te vinden. In het $p_{x}$-geval blijkt het paarpotentiaal $\Delta_{x}$ sterk onderdrukt te worden in de omgeving van het oppervlak. Een aanzienlijke energiekloof opent zich in de elektronische toestandsdichtheid enkel door de nabijheid van een bulk supergeleider. Er wordt getoond dat de nul-energiepiek in de toestandsdichtheid (DoS) ten aanzien van de oppervlakteruwheid zeer robuust is, terwijl een dergelijke piek in het geval van $d_{x} y$-symmetrie onderdrukt wordt. Dit effect wordt veroorzaakt door de stabiliteit van de paringstoestand met oneven frequentie, die wordt gegenereerd aan het oppervlak, ten opzichte van wanorde.

In het geval van de chirale $p_{x}+i p_{y}$-toestand blijft de tangenti $\Gamma \ll l e$ component van het paarpotentiaal $\Delta_{y}$ behouden aan de grens, terwijl de loodrechte component $\Delta_{x}$ sterk wordt onderdrukt, net als in het $p_{x}$-geval. De oppervlakteruwheid en de betreffende isotropisatie van de paaramplitude verminderen bovendien de magnitude van $\Delta_{y}$ aanzienlijk. Dit leidt tot de verschijning van een complexe meervoudige subkloofstructuur in het spectrum met toenemende oppervlakteruwheid. Daarnaast veroorzaken systemen met een eindige oppervlakteruwheid meer complexe spectra vergeleken met spiegeloppervlakten of oppervlakten met sterke wanorde. Met dit feit moet rekening gehouden worden bij de interpretatie van 
de resultaten van tunnelspectroscopie op onconventionele supergeleiders.

Alle verschijnselen die in hoofdstukken 1-4 beschouwd worden zijn gerelateerd aan de studie naar het nabijheidseffect. In hoofdstuk 1 is de voorafgaande beschouwing gegeven voor nabijheidseffecten tussen ferromagnetische en normale lagen binnen een zwakke schakel. Dit nabijheidseffect bepaalt de herverdeling van de stroom tussen geleidingskanalen door de junctie. Hoofdstukken 2-3 zijn gewijd aan de nabijheidseffecten van een dunne supergeleidende laag s met een F-laag en bulk S-elektroden. De wisselwerking met de F-laag onderdrukt de supergeleiding in de tussenlaag, terwijl de wisselwerking met de S-elektroden dit juist ondersteunt. Dit betekent dat de toestand van de middelste s-laag cruciaal is voor de werking van de gehele structuur. Tot slot is de aandacht in hoofdstuk 4 gericht op het nabijheidseffect tussen verschillende lobben van de p-golf-ordeparameter, die in connectie met elkaar staan door een ruw oppervlak. Alle natuurkundige systemen die hierboven genoemd zijn hebben veel gelijkenissen in hun beschrijvingen. De studie naar het nabijheidseffect in deze systemen maakt het mogelijk om effectievere en beter controleerbare apparaatjes voor supergeleidende elektronica te ontwikkelen. 


\section{List of Publications}

1. S. V. Bakurskiy, N. V. Klenov, T. Yu. Karminskaya, M. Yu. Kupriyanov and V.K. Kornev, "Current-Phase Relation in Josephson Junctions with Complex Ferromagnetic/Normal Metal Interlayers, Solid State Phenomena, 190, 401 (2012)

2. S. V. Bakurskiy, N. V. Klenov, T. Yu. Karminskaya, M. Yu. Kupriyanov and A. A. Golubov, "Josephson $\varphi$-junctions based on structures with complex normal/ferromagnet bilayer", Superconductor Science and Technology, 26, 015005 (2013)

3. I. V. Vernik, V. V. Bolginov, S. V. Bakurskiy, A. A. Golubov, M. Yu. Kupriyanov, V.V. Ryazanov and O. A. Mukhanov, "Magnetic Josephson Junctions with Superconducting Interlayer for Cryogenic Memory", IEEE Transactions on Applied Superconductivity, 23 (3), 1701208-1701208 (2013)

4. S. V. Bakurskiy, N. V. Klenov, I. I. Soloviev, V. V. Bolginov, V. V. Ryazanov, I. V. Vernik, O. A. Mukhanov, M. Yu. Kupriyanov, A. A. Golubov, "Theoretical model of superconducting spintronic SIsFS devices", Applied Physics Letters , 102 (19), 192603-192603-4 (2013)

5. T. E. Golikova, F. Hubler, D. Beckmann, N. V. Klenov, S. V. Bakurskiy, M. Yu. Kupriyanov, I. E. Batov, V. V. Ryazanov, "Critical current in planar SNS Josephson junctions", JETP letters, 96, 10, 668 (2013)

6. S. V. Bakurskiy, N. V. Klenov, I. I. Soloviev, M. Yu. Kupriyanov, A. A. Golubov, "Theory of supercurrent transport in SIsFS Josephson junctions", Physical Review B, 88, 14, $144519(2013)$

7. S. V. Bakurskiy, A. A. Golubov, M. Yu. Kupriyanov, K. Yada, Y. Tanaka, "Anomalous surface states at interfaces in p-wave superconductors", Physical Review B, 90, $6,064513(2014)$

8. I. I. Soloviev, N. V. Klenov, S. V. Bakurskiy, V. V. Bolginov, V. V. Ryazanov, M. Yu. Kupriyanov, A. A. Golubov, "Josephson magnetic rotary valve", Applied Physics 
Letters, 105 (24) 242601 (2014) 


\section{Acknowledgments}

Foremost I would like to express my deep gratitude to my scientific advisors Alexander Golubov and Mikhail Kupriyanov for comprehensive support and guidance in my work. Your advices specify my direction in the scientific world and demonstrate a wide range of options in it.

I would like to thank Hans Hilgenkamp for providing me an opportunity to start this $\mathrm{PhD}$ project in Twente

I am very grateful to my colleagues Nikolay Klenov and Igor Soloviev. Our collaboration always motivates me and generates spectrum of new ideas.

I have to thank many experimentalists for their perfect data and fruitful discussions. I especially would like to acknowledge Valeriy Ryazanov, Vitaliy Bolginov, Tatiana Golikova, Oleg Mukhanov, Igor Vernik, Edward Goldobin, Herman Kohlstedt and Nico Ruppelt.

I am also grateful to all ICE group of Twente University for the nice time, which I have spent in it. Especially I want to thank Ankur Dutt and Sebastian de Bone for their help with writing this thesis

In addition I would like to acknowledge fruitful discussions with my other colleagues. I should mention Yukio Tanaka, Keiji Yada, Shiro Kawabata, Yakov Fominov, Tatiana Karminskaya, Natalia Pugach, Horst Rogalla, Giampiero Pepe, Ondrej Vavra, Denis Heim, Angelina Burmistrova, Igor Devyatov, Anton Bespalov, Lize Serrier-Garcia, Victor Kornev, Nikolay Kolotinskiy and many others

Я хочу поблагодарить моих родителей, любимую девушку Лесю и друзей Геру, Сома и Васьгана за их всестороннюю поддержку и помощь.

And finally I would like to thank all Dutch and Russian taxpayers, who also supported this project. 